\title{
1 Corinthians
}

Incipit

Present: $5^{1} 545^{86175767778868889}$

incipit ad corinthios prima 86 ] incipit epistula ad corinthios $5^{7} 89$, om. 54 $5^{8} 7576$, incipit ad corinthios 61 , ad corinthios incipit 77 , ad corinthios prior incipit 78 , incipit epistula ad corinthios prima 88

1:1 Present: $5^{1} 545^{86164757677788688} 89$ AMst PEL CAr paulus

uocatus apostolus ] apostolus $7576 \mathrm{AMst}^{\mathrm{var}}$, apostolus uocatus $88^{*}$, uocatus apostolos CArvar

christi iesu ] iesu christi $89 \mathrm{AMst}^{\mathrm{var}} \mathrm{PEL}^{\mathrm{B}}$

per uoluntatem dei $54^{*}$ ] om. 64

et sosthenes 5158616478868889 ] abs. CAr

frater ] om. $\mathrm{PEL}^{\mathrm{B}}$, abs. CAr

1:2 Present: 5154586164757677788688 89 AMst PEL

ecclesiae dei 51545861777886

quae est corinthi $515458 \mathrm{AMst}^{\mathrm{var}} P E L^{A}$ ] quae est corinthis 61, om. 757677 $788689^{\mathrm{C}} \mathrm{AMst}^{\mathrm{ed}} \mathrm{PEL}^{\mathrm{B}}$, sụi $89^{*} ;$ + frater $\mathrm{PEL}^{\mathrm{B}}$

sanctificatis in (54)

christo iesu ] iesu 77 , christo $\mathrm{AMst}^{\mathrm{AD}}$

\pm quae est corinthi $75767886 \mathrm{AMst}^{\text {ed }} P E L^{B}$, quae est in corintho 77 , quae corinthi est 89

uocatis sanctis ] + uel sanctis 77

cum omnibus (PEL $\left.{ }^{\mathrm{AC}}\right)$ ] cum uniuersis $\mathrm{AMst}^{\mathrm{AD}}$, cum hominibus $\mathrm{PEL}^{\mathrm{A}}$ *

qui inuocant ] inuocantibus 7778

nomen domini nostri AMst ${ }^{\text {var }}$ ] nomen domini AMsted

iesu christi in omni loco ipsorum et nostro ] + uel nostrum 77

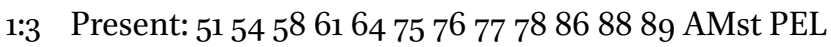

gratia uobis et pax a deo patre nostro 58

et ] om. 89

domino iesu christo $\left(75^{*}\right)$ AMst $^{\text {var }}$ ] domino nostro iesu christo 86 PEL $^{\text {var, }}$, iesu christo 89, christo iesu domino nostro AMst ${ }^{\text {ed }}$

1:4 Present: $5^{1} 545^{8} 616475767778868889$ AMst PEL CAr

gratias ago 58

(C) H.A.G. HOUGHTON ET AL., 2019 | DOI:10.1163/9789004390492_008

This is an open access chapter distributed under the terms of the prevailing CC-BY-NC License

at the time of publication. 
deo meo (61) ] domino meo 64

semper ] + uel omnino 77

pro ] pro uel de 77 , in $\mathrm{AMst}^{\mathrm{var}}$

uobis ] omnibus uobis 58

in $\left.\mathrm{AMst}^{\mathrm{AD}}\right]$ super $\mathrm{AMst}^{\mathrm{ed}}$, abs. CAr

gratia dei $\left.5^{8}\langle 64\rangle\right]$ gratia AMst ${ }^{\mathrm{var}}$, abs. CAr

quae data est 51545861768688 ] abs. CAr; + uel data 77

uobis ] mihi $\mathrm{PEL}^{\mathrm{B}}$, abs. CAr

in christo iesu ] in christo AMst ${ }^{\mathrm{var}}$, abs. CAr; + christo 89*

1:5 Present: 515458616475767778868889 AMst PEL

quia ] quoniam $617576777886 \% 89 \mathrm{PEL}^{\mathrm{B}}$, qui AMst ${ }^{\mathrm{var}}$

in omnibus $\langle 64\rangle]$ in omni 77

diuites facti $\left(58^{*}\right) \mathrm{AMst}^{\mathrm{AD}}$ ] locupletati $6175767889 \mathrm{PEL}^{\mathrm{B}}$, ditati $64 \mathrm{AMst}^{\mathrm{ed}}$, locupletati uel ditati 77 , lac. 86

estis in illo $\mathrm{AMst}^{\mathrm{var}}$ ] estis in ipso $61 \mathrm{AMst}^{\mathrm{ed}} \mathrm{PEL}^{\mathrm{B}}$, lac. 86

in omni uerbo ] omni uerbo 75 , lac. 86

et in ] et 6164757677 , lac. 86

omni scientia 54 ] scientia 61, lac. 86

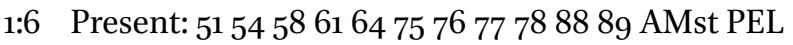

sicut ] + et $\mathrm{AMst}^{\mathrm{A}}$

testimonium christi ] testimonium dei 7778

confirmatum est ] + uel fundatum est 77

in uobis

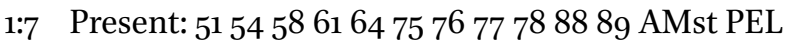

ita ut

nihil uobis desit $5154 \mathrm{AMst}^{\mathrm{AD}}$ ] uobis nihil desit 58 , non indigeatis 61757689 , nihil desit uobis 64 , uos non indigeatis 7778 , uobis in nullo desit AMst ${ }^{\text {ed }}$, nil uobis desit PEL var

in ulla AMst ${ }^{\mathrm{AD}}$ ] in nulla $647576 * 89 \mathrm{PEL}^{\mathrm{A}}$, aliqua $\mathrm{AMst}^{\text {ed }}$

gratia expectantibus 58 ] gratia expectantes 6164757689 PEL $^{\mathrm{A}}$; + uel expectantes 77

reuelationem domini 58

nostri ] om. PELA*

iesu christi ] iesu christo 78

1:8 Present: 51545861647576777888 89 AMst PEL

qui et ] qui et [3] $89^{*}$ 
confirmabit uos ] confirmauit uos $54^{*} 6475767778$ 88* 89 AMst $^{\text {var }}$ PEL $^{\mathrm{A}}$ usque ad finem ] usque in finem $5154586164757778 \mathrm{AMst}^{\mathrm{var}} \mathrm{PEL}^{\mathrm{var}}$, usque in fine 76

sine crimine in 61

die aduentus ] aduentum $617576 \mathrm{AMst}^{\mathrm{ed}}$, diem 64, aduentu $7778 \mathrm{AMst}^{\mathrm{var}}$, die $\mathrm{AMst}^{\mathrm{AD}}$

domini nostri iesu christi

1:9 Present: 51545861647576777888 89 AMst PEL

fidelis ] + autem 58 , + est AMst ${ }^{\text {ed }}$

deus per quem uocati estis in

societatem ] communionem $\mathrm{AMst}^{\mathrm{R}}$, societate $\mathrm{PEL}^{\mathrm{B}}$

filii eius 6475 ] filii 61 AMst $^{\text {var }}$

iesu christi domini nostri ] domini nostri iesu christi $61 \mathrm{AMst}^{\mathrm{var}} \mathrm{PEL}^{\mathrm{B}}$, iesu christi nostri 89, om. PEL ${ }^{\text {var }}$

1:10 Present: 51545861647576777888 89 AMst PEL teS SPE

obsecro autem $5^{8}$ ] obsecro AMst ${ }^{\text {var }}$ TES $^{\text {var }}$

uos fratres per

nomen ] misericordiam TE ${ }^{\mathrm{var}}$

domini nostri iesu christi $\langle 64\rangle$ SPE ${ }^{\text {var }}$ ] iesu christi domini nostri 7577 78, christi iesu domini nostri 76 , domini nostri iesu 89 , domini iesu christi $\mathrm{TES}^{\mathrm{var}} \mathrm{SPE}^{\mathrm{ed}}$

ut id ipsum ] ut ipsum $89^{*} \mathrm{AMst}^{\mathrm{A} *} \mathrm{SPE}^{\mathrm{var}}$, ut et ipsum PEL ${ }^{\mathrm{var}}$, ut ipsud $\mathrm{TES}^{\mathrm{var}}$ dicatis omnes ] iudicatis omnes $\mathrm{TES}^{\mathrm{var}}$

et non ] ut non $89 \mathrm{AMst}^{\mathrm{A}} \mathrm{TES}^{\mathrm{var}}$

sint ] om. $88^{*}$, sit AMst ${ }^{\mathrm{var}} \mathrm{TES}^{\mathrm{var}}$

in uobis ] inter uos AMst ${ }^{\text {var }}$

scismata $88^{*}$ ] scissurae 757689

sitis autem ] sitis enim TES ${ }^{\text {var }}$, sitis TES $S^{\text {var }}$

perfecti TES $S^{\text {var }}$ ] perfecta 88, conpositi TES $S^{\text {ed }}$, non positi TES $S^{\text {var }}$

in eodem sensu ] in eodem intellectum $64, \mathrm{om} .76^{*}$, in eundem sensum TES var

et in eadem ] et eodem TES ${ }^{\text {var }}$

sententia $5888^{\text {alt }} \mathrm{AMst}{ }^{\mathrm{var}}$ ] scientia $5^{1} 5^{\mathrm{C}} 757^{6} 777888 \mathrm{AMst}^{\mathrm{ed}} \mathrm{PEL}^{\mathrm{B}} \mathrm{SPE}^{\mathrm{var}}$, $\mathrm{s}\left[3^{-4}\right]$ entia $54^{*}$, sententiam 64 , consilio TE ${ }^{\mathrm{var}}$

1:11 Present: 5154586164757677788889 AMst PEL SPE

significatum est ] nuntiatum est 64 , perlatum est $75767789 \mathrm{AMst}^{\mathrm{ed}}$, reuelatum est 78 , relatum est $\mathrm{AMst}^{\mathrm{R}}$, indicatum est PEL 
enim ] om. $\mathrm{AMst}^{\mathrm{AD}}$, autem $S P E^{\mathrm{var}}$

mihi de uobis $5^{1} 545^{8}$ ] de uobis 76

fratres mei ] fratres 61757689 AMst PEL $^{\mathrm{A}}$

ab ] ad $75^{*}$

his $\left.5^{8}\right]$ his [1-3] $54^{*}$, iis AMst $^{\mathrm{R}}$

qui sunt ] om. 89

chloes 51545861648889 ] coloes $88^{\mathrm{gl}}$

quia AMst ${ }^{\mathrm{AD}}$ ] quod $5^{1} 617576777889 \mathrm{AMst}^{\mathrm{ed}} \mathrm{PEL}^{\mathrm{B}} \mathrm{SPE}^{\mathrm{var}}$

contentiones 5861

inter uos sunt $\mathrm{AMst} \mathrm{t}^{\mathrm{AD}}$ ] sint inter uos $5^{1} 89 \mathrm{AMst}^{\mathrm{ed}}$, sunt inter uos 545888 , inter uos sint $617778 \mathrm{AMst}^{\mathrm{var}} \mathrm{PEL}^{\mathrm{B}}$, sunt in uobis 64

1:12 Present: 5154586164757677788889 AMst PEL SPE

hoc autem dico AMstrar ] dico autem hoc $75767789 \mathrm{AMst}^{\mathrm{ed}} \mathrm{PEL}^{\mathrm{B}}$; + fratres 58

quod ] quoniam 61 $\mathrm{PEL}^{\mathrm{B}}$, quia 6475767778

unusquisque uestrum dicit $\left(75^{*}\right)$ ] singuli ex uobis dicunt $61 \mathrm{PEL}^{\mathrm{B}}$, unusquisque dicat $S P E^{\text {var }}$

ego quidem ] ego siquidem $S P E^{\mathrm{var}}$

sum ] om. AMst ${ }^{\mathrm{var}}$, sunt $\mathrm{PEL}^{\mathrm{B}}$

pauli ego autem apollo ego 5861

uero ] autem 75767778

cephae $515458647576\langle 78\rangle$

ego autem christi ] ego uero christi 77

1:13 Present: 51545861647576777888 89 AMst PEL SPE

diuisus est 61$]+\operatorname{ergo} 54^{\mathrm{C}} 5^{8}$

christus numquid paulus 77

crucifixus est pro uobis ] pro uobis crucifixus est 64, crucifixus est pro nobis

PEL ${ }^{\mathrm{B}}$

aut in nomine pauli

baptizati estis $5467^{c}$ ] crucifixi estis $61^{*}$

1:14 Present: 5154586164757677788889 AMst PEL CAr

gratias ago deo 58 ] gratias ago CAr; + meo $51545861 \mathrm{AMst}^{\mathrm{var}} \mathrm{PEL}^{\mathrm{var}}$

quod neminem $\langle 64\rangle$ ] quia neminem $5^{1} 64$

uestrum baptizaui $545^{8}$ ] baptizaui uestrum 61 , lac. 64, uestrum baptizaue$\operatorname{rim} 78$

nisi crispum 76 ] lac. 64, abs. CAr

et gaium ] et caium $\mathrm{AMst}^{\mathrm{R}}$, abs. $\mathrm{CAr}$ 
1:15 Present: 51545861647576777888 89 AMst PEL

ne quis ] ut ne quis 7778

dicat quod ] dicat quia $6489 \mathrm{AMst}^{\mathrm{AD}}$

in nomine meo ] in meo nomine 77

baptizati sitis $5^{c}$ ] baptizati estis $51767^{1} 88^{\mathrm{C}} \mathrm{AMst}^{\mathrm{var}} \mathrm{PEL}^{\mathrm{var}}$, [3] tiza[2] sitis $54^{*}$, baptizati essetis 58 , baptizaui $6164757789 \mathrm{AMst}^{\mathrm{ed}} \mathrm{PEL}^{\mathrm{B}}$, baptizaue$\operatorname{rim} 78^{\mathrm{gl}}$

1:16 Present: 51545861647576777888 89 AMst PEL

baptizaui autem et stephanae 515458616475767788

domum ] domi 75

ceterum $6 r \mathrm{AMst}^{\mathrm{var}}$ ] ceterorum AMsted; + ạụtẹm $54^{\mathrm{C}}$

nescio si quem AMst ${ }^{\mathrm{var}}$ ] nescio s[2] quem $54^{*}$, nescio si aliquem 6164 AMsted

alium AMst ${ }^{\mathrm{var}}$ ] om. $5^{1} 617576777889 \mathrm{AMst}^{\mathrm{ed}} \mathrm{PEL}^{\mathrm{B}}$; + uestrum $51586188^{*}$ 89 PEL $^{B}$

baptizauerim $545^{8} 61 \mathrm{AMst}^{\mathrm{var}}$ ] baptizaui $6475767789 \mathrm{AMst}^{\mathrm{ed}} \mathrm{PEL}^{\mathrm{B}}$

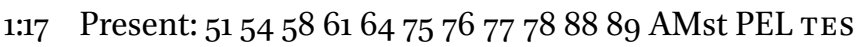

non enim ] om. TES

misit me christus 61 ] christus misit me 64

baptizare sed euangelizare $545^{8} 61$ ] adnuntiare TES

non in sapientia uerbi 54 ] non in sapientiae uerbi 88 , non in sermonis sapientia TES ${ }^{\text {ed }}$, non in sermone sapientiae TES ${ }^{\mathrm{var}}$

ut non euacuetur (89) AMst ${ }^{\text {var }}$ ] ut euacuetur 6178 , ne euacuetur $64 \mathrm{AMst}^{\mathrm{ed}}$, et non euacuetur $\mathrm{PEL}^{\mathrm{A} *}$, ne inanis fiat TES ${ }^{\mathrm{ed}}$, ne inane fiat $\mathrm{TES}^{\mathrm{var}}$ crux christi

1:18 Present: $5^{1} 545^{8} 61647576777888$ 89 AMst PEL tes

uerbum enim crucis ] uerbum autem crucis 61 , uerbum crucis TE $S^{\text {var }}$

pereuntibus quidem stultitia est $\left.51545^{8}\left(\mathrm{PEL}^{\mathrm{A}}\right)\right]$ pereuntibus quidem stultitia $\left(61^{*}\right)^{\mathrm{C}}$, pereuntibus stultitia est $6475^{*} 7689$, quidem pereuntibus stultitia est 77 , stultitia est his qui pereunt TES ${ }^{\text {ed, }}$, stultitia est eis qui pereunt TES $S^{\text {var }}$

his autem qui salui fiunt 58 ] saluatis uero 77 , his uero qui salui fiunt 78 , his enim qui salui fiunt $\mathrm{AMst}^{\mathrm{D}}$, salus autem futuris $\mathrm{AMst}^{\mathrm{A}}$, his autem qui saluantur TES ${ }^{\text {ed }}$, his autem qui saluabuntur TES $S^{\text {var }}$

id est nobis $\mathrm{AMst}^{\mathrm{D}}$ ] id est uobis 58 , om. $61647576777^{8} 88^{\mathrm{C}} 89 \mathrm{AMst}^{\mathrm{ed}} \mathrm{PEL}^{\mathrm{B}}$

TES, nobis $\mathrm{AMst}^{\mathrm{A}}$

uirtus dei est 
1:19 Present: $5^{1} 545^{8} 61647576777888$ 89 AMst PEL TES

scriptum est enim 6488 ] sicut scriptum est 58 , et scriptum est enim TES var $^{\text {val }}$

\pm in esaia $54^{c}$, in esaia propheta 61

perdam sapientiam $545^{8}$

sapientium et 58 ] et $76^{*}$

prudentiam $\left.58 \mathrm{AMst}^{\mathrm{A}} \mathrm{PEL}^{\text {var }}\right]$ intellectum $61 \mathrm{AMst}^{\mathrm{ed}} \mathrm{PEL}^{\mathrm{B}}$, prudentia 64

PEL ${ }^{\mathrm{AC}}$, om. PEL ${ }^{\mathrm{A}}$; + uel intellectum uel sensum 77

prudentium reprobabo 58 ] prudentium perprobabo 89

1:2O Present: 51545861647576777888 89 AMst PEL TES SPM

ubi ] + enim SPM var

sapiens ubi scriba ] sapientes et ubi scriba TE $S^{\text {var }}$

ubi conquisitor 61 ] ubi inquisitor $5^{1}$

huius saeculi $5154\left(5^{8}\right) 617688$ TES $\left.^{\text {var }}\right]$ saeculi huius 7778 TES $^{\text {ed }}$, huius mundi AMst ${ }^{\mathrm{A}}$ TES $^{\text {var }}$ SPM $^{\text {var }}$

nonne

stultam AMst ${ }^{\text {var }}$ TE $S^{\text {var }}$ ] stultum 61 AMst $^{\text {ed }}$, stulta $75^{*}$, infatuabit TES ${ }^{\text {var }}$, stultitiam TES $S^{\text {var }}$, infatuauit TES $S^{\text {ed }}$

fecit deus TES $S^{\text {var }}$ ] fecit $61^{*}$ TES $S^{\text {var }}$, deus fecit $78^{*}$, deus TES $S^{\text {ed }}$

sapientiam ] sapientia 64

huius mundi SPM ${ }^{\mathrm{var}}$ ] mundi huius 7778 , saeculi TES ${ }^{\mathrm{var}}$, mundi SPM${ }^{\text {ed }}$

1:21 Present: $5^{1} 545^{861647576777888} 89$ AMst PEL TES

nam quia $78 \mathrm{AMst}^{\mathrm{var}}$ ] nam quoniam $64 \mathrm{AMst}^{\mathrm{ed}}$, quia nam $7^{\mathrm{gl}}$, quoniam enim $\mathrm{AMst}^{\mathrm{AD}}$, quoniam quidam TES

in dei sapientia 58 ] in sapientia dei 6175767778 AMst $^{\text {TES }}{ }^{\text {ed }}$, deus sapientiam 89, in dei sapientiam PEL ${ }^{B}$, in sapientiam mundi TE $S^{\text {var }}$, per sapientiam domini TES ${ }^{\mathrm{var}}$, sapientia dei TES ${ }^{\mathrm{var}}$

non cognouit ] cognouit TES $S^{\text {var }}$

mundus ] hic mundus $586188^{\mathrm{C}} 89 \mathrm{PEL}^{\mathrm{B}} \mathrm{TES}^{\mathrm{var}}$, mundum 75

per sapientiam 5458 ] + suam $61 \mathrm{TES}^{\mathrm{var}}$

deum ] domini $5^{8} 75 \mathrm{PEL}^{\mathrm{B}} \mathrm{TES}^{\mathrm{var}}$, dominum 89, dei AMst ${ }^{\mathrm{var}}$, deo PEL ${ }^{\mathrm{var}}$, om. TES var

placuit TES $S^{\text {var }}$ ] conplacuit TES $S^{\text {ed }}$

deo per stultitiam praedicationis 5154586176777888

saluos facere credentes ] saluare credentes 7778 TES

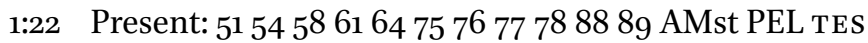

quoniam ] + quidem $6164757689 \mathrm{AMst}^{\mathrm{AD}} \mathrm{TES}^{\mathrm{var}}$ 
et PEL ${ }^{\text {var }}$ ] om. $54617576777^{8} 88^{\mathrm{C}} 89$ AMst PEL ${ }^{\mathrm{AB}}$ TES

iudaei signa 515458617688

petunt $\left.\left(\mathrm{PEL}^{\mathrm{B}}\right) \mathrm{TES}^{\mathrm{var}}\right]$ desiderant TES ${ }^{\mathrm{ed}}$

et graeci 5154586176777888

sapientiam $5458 \mathrm{TES}^{\mathrm{var}}$ ] prudentiam TES $\mathrm{Sed}^{\mathrm{ed}}$

quaerunt 515458617688 ] requirunt $\mathrm{AMst}^{\mathrm{var}}$

1:23 Present: 5154586164757677788889 AMst PEL TES SPM nos autem praedicamus 51545861767778

christum ] iesum christum 61, om. TES var $^{2}$

crucifixum iudaeis quidem scandalum gentibus autem stultitiam $51545^{8}$

$6176\left(77^{*}\right) 88$ ] om. sPM ${ }^{\text {var }}$

1:24 Present: 5154586164757677788889 AMst PEL TES SPM

ipsis ] ipsi 77 , om. $88^{*} \mathrm{SPM}^{\mathrm{var}}$

autem $\mathrm{AMst}^{\mathrm{AD}}$ ] uero $64 \mathrm{AMst}^{\mathrm{ed}} \mathrm{PEL}^{\mathrm{B}}$, om. $88^{*} \mathrm{SPM}^{\mathrm{var}}$, autem uero TES $\mathrm{S}^{\mathrm{var}}$

uocatis ] om. $88^{*} \mathrm{PEL}^{\mathrm{B}} \mathrm{SPM}^{\mathrm{var}}$, uocitis TES ${ }^{\mathrm{var}}$

iudaeis $515458617688^{C}$ ] om. $88^{*} \mathrm{SPM}^{\mathrm{var}}$

atque graecis $51545^{8} 617888^{c}$ ] et graecis 64757677 TES SPM ${ }^{\text {ed }}$, om. $88^{*}$

$\mathrm{SPM}^{\mathrm{var}}$

christum ] om. $88^{*}$ TES $^{\text {var }}$ SPM $^{\text {var }}$; + praedicamus 58

dei uirtutem et dei sapientiam ] om. $88^{*}$

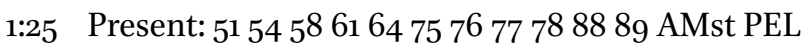

quia quod stultum est dei sapientius est $545^{8}$

hominibus ] quam homines 64

et quod infirmum est $\mathrm{AMst}^{\mathrm{R}}$ ] et infirmum $757689 \mathrm{AMst}^{\text {ed }}$, et quod infirmum $\mathrm{AMst}^{\mathrm{AD}}$, et infirmum est $\mathrm{PEL}^{\text {var }}$

dei fortius est ] dei fortius AMst ${ }^{\mathrm{AD}}$

hominibus ] quam homines 64

1:26 Present: 515458616475767778848889251 AMst PEL SPM

uidete $\mathrm{AMst}^{\mathrm{AD}}$ ] fratres uidete 84251 , intuemini $\mathrm{AMst}^{\mathrm{ed}}$

enim SPM ${ }^{\mathrm{var}}$ ] ergo 7778 , om. $84251 \mathrm{SPM}^{\text {ed }}$

uocationem uestram fratres $58 \mathrm{AMst}^{\mathrm{AD}}$ ] uocationem uestram 84251 , fratres

uocationem uestram AMsted, uocationem fratres PEL ${ }^{\mathrm{B}}$

quia ] quoniam 5875767789 , abs. 84

non multi sapientes ] abs. $84 ;+$ sunt 89

secundum carnem ] abs. $84 ;+$ non multi fortes $5^{8} \mathrm{PEL}^{\mathrm{B}}$

non multi ] om. $77 \mathrm{PEL}^{\mathrm{B}}$, abs. 84 
potentes 251 ] om. $777^{\mathrm{C}} \mathrm{PEL}^{\mathrm{B}}$, prudentes AMst ${ }^{\mathrm{var}}$, abs. 84 non multi nobiles ] abs. 84

1:27 Present: $5^{1} 545^{8} 6164757677788889$ 251 AMst PEL SPM sed quae stulta sunt $5154587688 \mathrm{AMst}^{\mathrm{AD}}$ ] sed quae sunt stulta 61 , sed stulta $64 \mathrm{AMst}^{\mathrm{ed}}$, sed quae stulta 251

mundi elegit ] om. 77, mundi eligit PEL ${ }^{\mathrm{A}}$, huius mundi elegit SPM deus AMst ${ }^{\text {var }}$ ] om. 77 AMst ${ }^{\text {ed }}$

ut confundat sapientes et ] om. $777^{\mathrm{C}} 89^{*}$

infirma ] om. $777^{\mathrm{C}} 89^{*}$, quae infirma sunt $\mathrm{AMst}^{\mathrm{AD}}$, infirmum $\mathrm{PEL}^{\mathrm{B}}$ mundi elegit ] om. $78^{\mathrm{C}} 89^{*}$, huius mundi elegit $89^{\mathrm{C}}$ deus ] deo $75^{*}$, om. $78^{\mathrm{C}} 89$, dominus $88^{*}$ ut confundat $\langle 64\rangle$ ] ut confudạt 58 ; + sapientia 61 fortia 54251 ] lac. 64 , fortiora $75\langle 76\rangle$

1:28 Present: $5^{1} 545^{8} 6164757677788889$ 251 AMst PEL SPM et ignobilia ] lac. 64 mundi AMst ${ }^{\mathrm{R}}$ ] huius mundi $6175^{\mathrm{C}}\langle 76\rangle 89 \mathrm{AMst}^{\mathrm{ed}} \mathrm{PEL}^{\mathrm{B}}$, lac. 64, huius modi $75^{*}$, om. 78 sPM et contemptibilia elegit $(61)\langle 64\rangle(75) 251\left(\mathrm{PEL}^{\mathrm{B}}\right)$

deus ] om. SPM et $\mathrm{AMst}^{\mathrm{var}}$ SPM $^{\mathrm{var}}$ ] om. 75767789 AMst $^{\mathrm{ed}} \mathrm{PEL}^{\mathrm{B}}$ SPM $^{\text {ed }}$, ut SPM ${ }^{\mathrm{var}}$ quae $547688 \mathrm{PEL}^{\text {var }}$ ] ea quae $51586164 \mathrm{AMst}^{\mathrm{var}} \mathrm{PEL}^{\mathrm{AB}} \mathrm{SPM}^{\mathrm{var}}$ non sunt ] [non sunt] tamquam quae sint 64 ut ea quae sunt 515458617688 ] ut quae sunt $\langle 64\rangle \mathrm{PEL}^{\mathrm{B}} \mathrm{SPM}$ destrueret $6175(88)$ ] euacuet $\langle 64\rangle \mathrm{SPM}^{\text {var }}$, destruet 77 , destituat SPM ${ }^{\text {ed }}$

1:29 Present: 5154586164757677788889251 AMst PEL sPM ut non ] ut 54 * glorietur omnis caro $\left(54^{*}\right)\langle 64\rangle\left(78^{*}\right)$ in conspectu eius $\mathrm{AMst}^{\mathrm{R}} \mathrm{SPM}^{\mathrm{var}}$ ] coram deo $64757689 \mathrm{AMst}^{\mathrm{AD}} \mathrm{SPM}^{\mathrm{ed}}$, in conspectu uel coram dei uel deo 77 , coram illo 78 , in conspectu illo $7^{\mathrm{gl}}$, in conspectu dei $\mathrm{AMst}^{\mathrm{ed}} \mathrm{PEL}^{\mathrm{B}}$, coram domino $\mathrm{PM}^{\mathrm{var}}$

1:30 Present: 5154586164757677788889 251 AMst PEL sPE ex ipso autem AMst ${ }^{\text {var }}$ ] ex ipso enim $54 \mathrm{AMst}^{\text {ed }}$ uos estis ] uos uocati estis $5^{8}$ in christo iesu $\langle 64\rangle$ qui factus est ] quia factus est $75^{*}$, qui factus 78

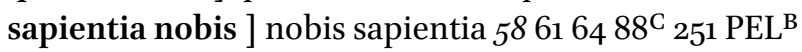


a deo AMst $^{\text {var }}$ ] dei 58 , om. AMst ${ }^{\text {ed }}$

et iustitia et sanctificatio et redemptio $51545^{8}(64)(88)$

1:31 Present: $5^{1} 545^{8} 6164757677788889$ 251 AMst PEL CAr SPE

ut ] om. 54, abs. CAr

quemadmodum ] sicut $\mathrm{AMst} \mathrm{AD}^{\mathrm{AD}}, a b s$. $\mathrm{CAr}$

scriptum est 64251 ] abs. CAr

qui gloriatur in

domino glorietur ] deo glorietur $\mathrm{SPE}^{\mathrm{var}}$

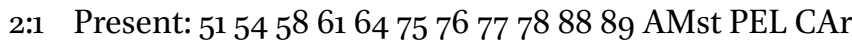

et ego ] ego CAr

cum uenissem ad uos fratres 61 ] ueniens ad uos fratres 647576777889

$\mathrm{AMst}^{\mathrm{AD}} \mathrm{PEL}^{\mathrm{B}}$, fratres cum uenissem ad uos CAr

ueni non ] uenio non PEL var, abs. CAr

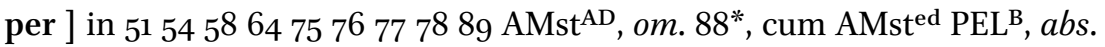

CAr

sublimitatem ] sublimitate $5^{1} 5^{8} 75767778$ 89, elationem 64, eminentia

AMst PEL ${ }^{\mathrm{B}}$, abs. CAr

sermonis ] uerbi $64 \mathrm{AMst}^{\mathrm{AD}}$, sermonum PEL ${ }^{\mathrm{var}}$, abs. CAr

aut sapientiae 51545876 ] abs. CAr

adnuntians uobis $51545^{8} 78\left(88^{*}\right) \mathrm{AMst}^{\mathrm{AD}}\left(\mathrm{PEL}^{\mathrm{A}}\right)^{\mathrm{C}}$ ] praedicans uobis 61

$\mathrm{AMst}^{\mathrm{ed}} P E L^{B}$, abs. CAr

testimonium ] mysterium 6764 AMst $P E L^{B}$, abs. CAr

christi PELvar ] dei 647576777889 AMst PEL ${ }^{\mathrm{A}}$, om. $\mathrm{PEL}^{\mathrm{B}}$, abs. CAr

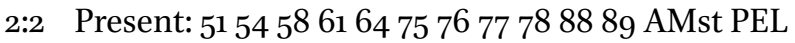

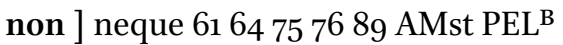

enim ] om. AMst ${ }^{\text {var }}$

iudicaui ] dicam 58

scire me ] me scire 5458616489 AMst $^{\mathrm{var}} \mathrm{PEL}^{\mathrm{B}}$, scire 757677

aliquid inter uos 54 ] aliquid in uobis 647778 , aliquid intra nos $\mathrm{PEL}^{\mathrm{B}}$

nisi iesum christum $\langle 64\rangle \mathrm{AMst}^{\mathrm{AD}}$ ] nisi christum iesum $5^{1} 5^{8} 61777889$

$\mathrm{AMst}^{\mathrm{ed}} \mathrm{PEL}^{\mathrm{B}}$, nisi christum AMst ${ }^{\mathrm{var}}$

et hunc crucifixum ] et hunc esse inter uos crucifixum AMst ${ }^{\text {var }}$

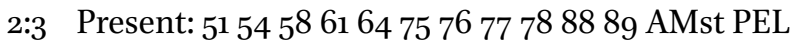

et ego in

infirmitate $\left(78^{*}\right)$ ] infirmitatem $64^{*}$

et timore ] et in timore $5^{8} 757688$, et timorem $64^{*}, o m$. $\mathrm{PEL}^{\mathrm{B}}$ 
et tremore multo fui AMst ${ }^{\text {var }}$ ] multo fui AMst ${ }^{\text {ed }}$

apud uos $5154 * 5875]$ ad uos 64 , apud uel ad uos 77

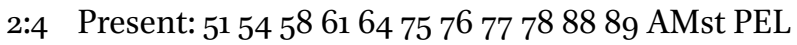

et sermo meus ] et uerbum meum $\mathrm{AMst}^{\mathrm{AD}}$

et praedicatio mea non in $51545^{8} 61\langle 64\rangle 767788$

persuasibilibus ] persuasione $617576777889 \mathrm{AMst}^{\mathrm{ed}}$, suasoriis 64 , suasione $\mathrm{AMst}^{\mathrm{AD}}$

sapientiae 7677 ] humanae sapientiae $5154586188 \mathrm{PEL}^{\mathrm{B}}$

uerbis $\mathrm{AMst}^{\mathrm{A}}$ ] om. $61777889 \mathrm{AMst}^{\mathrm{ed}}$, sermones 75 76, uerborum $\mathrm{AMst}^{\mathrm{R}}$, uerbi $\mathrm{AMst}^{\mathrm{D}}$

sed ] om. AMst

in ostensione $\left.54 \mathrm{AMst}^{\mathrm{var}} P E L^{A *}\right]$ ad ostensionem AMsted, in ostentatione PEL ${ }^{A C}$

spiritus et

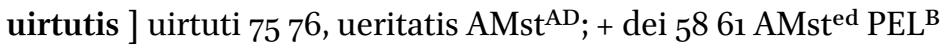

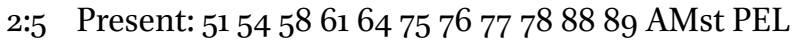

ut $\mathrm{AMst}^{\mathrm{var}}$ ] ut esset 78 , et 89 , om. AMst ${ }^{\text {ed }}$

fides uestra non $\left.\langle 64\rangle \mathrm{AMst}^{\mathrm{var}}\right]$ om. AMst $\mathrm{od}^{\mathrm{ed}}$

sit $\mathrm{AMst}^{\mathrm{var}}$ ] om. $617778 \mathrm{AMst}^{\mathrm{ed}}$

in sapientia hominum $5458 \mathrm{AMst}^{\mathrm{var}}$ ] in sapientiam hominum 757689 , om.

AMst $^{\text {ed }}$

sed in uirtute $\mathrm{AMst}^{\mathrm{var}}$ ] sed in potentia 64, om. AMst ${ }^{\mathrm{ed}}$

dei $\mathrm{AMst}^{\mathrm{var}}$ ] om. AMsted; + sit $61 \mathrm{PEL}^{\mathrm{B}}$

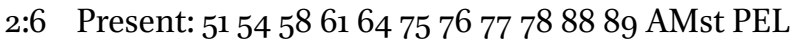

sapientiam $545^{8}$ ] sapientia $64^{*}$

autem $\mathrm{AMst}^{\mathrm{var}}$ ] enim $\mathrm{AMst}^{\mathrm{ed}}$, om. $\mathrm{PEL}^{\mathrm{B}}$

loquimur inter perfectos ] loquimur apud perfectos $61 \mathrm{PEL}^{\mathrm{B}}$

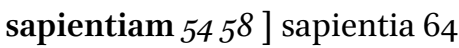

uero $\mathrm{AMst}^{\mathrm{R}}$ ] autem $54616475767778 \mathrm{AMst}^{\mathrm{ed}} \mathrm{PEL}^{\mathrm{B}}$, ergo AMst ${ }^{\mathrm{AD}}$

non huius saeculi 51545876 ] non huius saeculi huius $77^{*}$, non saeculi huius $77^{\mathrm{C} *} 78$

neque principum ] om. 7778

huius saeculi $51545^{8} 76$ ] om. 7778 , huius mundi AMst ${ }^{\mathrm{AD}}$, eius AMst ${ }^{\text {var }}$ qui destruuntur 61 ] qui euacuatur 64 , quae destruitur 78

2:7 Present: 51545861647576777888 89 AMst PEL sPM

sed loquimur dei sapientiam $545^{8}$ ] sed loquimur sapientiam dei SPM $^{\text {var, }}$, sapientiam autem loquimur SP $\mathrm{M}^{\text {var }}$ 
in ] et $75^{*}$

mysterio 5461 ] ministerio $5^{8} \mathrm{PEL}^{\text {var, }}$, mysterium $64 ;+$ christi $58,+$ dei PEL $^{\mathrm{B}}$

quae abscondita est 51545861 (88) SPM ${ }^{\mathrm{var}}$ ] quae in abscondito est 647576

89 SPM $^{\text {ed }}$, absconditam 7778

quam ] quem 61, quae $\mathrm{AMst}^{\mathrm{A}}$

praedestinauit 515458617677788889 ] praefiniuit 64

deus ante saecula in gloriam nostram 5154586176

2:8 Present: 51545861647576777888 89 AMst PEL SPM

quam nemo principum $\mathrm{AMst}^{\mathrm{var}}$ ] quem non principum $\mathrm{AMst}^{\mathrm{ed}}$

huius saeculi $51545^{8} 6178$ ] saeculi huius 757677

cognouit ] cognouis $89^{*}$

si enim cognouissent numquam $6 r\langle 64\rangle 77$

dominum ] deum $\mathrm{AMst}^{\mathrm{A}}$

gloriae $51545888 \mathrm{PEL}^{\mathrm{var}} \mathrm{SPM}^{\mathrm{var}}$ ] maiestatis 61 (75) $7689 \mathrm{AMst}^{\mathrm{PEL}}{ }^{\mathrm{AB}} \mathrm{SPM}^{\mathrm{ed}}$, gloriae uel maiestatis 77

crucifixissent

2:9 Present: 51545861647576777888 89 AMst PEL

sed sicut scriptum est 64 ] sed scriptum sicut est $54^{*}$, sed sicut est scriptum $54^{\mathrm{C}}$

quod ] qu $54^{*}$, quae 77

oculus non uidit 54

nec auris audiuit 75 ] et auris non audiuit 6477, om. $88^{*}$

nec in cor hominis ascendit $\langle 64\rangle]$ nec in cor hominis ascendet $54^{*}$, et in cor hominis non ascendit 7576777889

quae 515458617688 ] hoc AMst $^{\mathrm{R}}$

praeparauit deus $51545861\langle 64\rangle 77$ (88)

his qui diligunt illum $5^{8}$ ] diligentibus se $5^{1} 61777_{8} \mathrm{PEL}^{\mathrm{B}}$, his qui diligunt eum $54 \mathrm{AMst}^{\mathrm{var}}$, lac. 64, diligentibus eum $757689 \mathrm{AMst}^{\mathrm{ed}}$

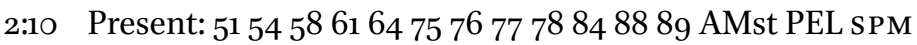

nobis ] lac. 64 , fratres nobis 84

autem $\mathrm{AMst}^{\mathrm{ADR}}$ ] lac. 64, om. 84, uero $\mathrm{AMst}^{\mathrm{ed}}$, enim SPM

reuelauit $\left(88^{*}\right) \mathrm{SPM}^{\mathrm{var}}$ ] praeparauit $61^{*}$, lac. 64 , reuelat $\mathrm{SPM}^{\mathrm{ed}}$

deus ] om. PEL ${ }^{\text {var }}$ SPM $^{\text {var }}$

per ] propter $\mathrm{PEL}^{\mathrm{B}}$

spiritum suum ] spiritum sanctum 51

spiritus enim ] nam spiritus SPM, abs. 84 
omnia scrutatur $\langle 64\rangle(76) \mathrm{SPM}^{\mathrm{var}}$ ] omnia scrutat $75 \mathrm{SPM}^{\mathrm{ed}}$, abs. 84

etiam ] et $77, a b s .84$

profunda dei ] altitudinem dei $64 \mathrm{PEL}^{\mathrm{B}} \mathrm{SPM}^{\mathrm{var}}$, alta dei $75767889 \mathrm{AMst}^{\mathrm{AD}}$ $\mathrm{SPM}^{\mathrm{ed}}$, altitudines uel alta dei $77, a b s .84$

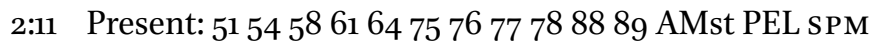

quis enim ] quis autem $\mathrm{AMst} \mathrm{AD}^{\mathrm{AD}}$

scit hominum ] hominum scit $61 \mathrm{AMst}^{\mathrm{R}} \mathrm{PEL}^{\mathrm{B}}$

quae sint hominis 5888 ] quae sunt hominis 515464777889 AMst $^{\mathrm{AD}} \mathrm{PEL}^{\text {var }}$

SPM, quae hominis sunt $61 \mathrm{PEL}^{\mathrm{B}}$, quae sit hominum 75 , quae $\sin [t]$ homi-

num 76 , ea quae sunt hominis AMsted, ea quae sint hominis AMstrar, hominis abscondita $\mathrm{AMst}^{\mathrm{R}}$

\pm quae in ipso sunt $89 \mathrm{SPM}^{\mathrm{ed}}$, quoniam in ipso sunt $\mathrm{PEL}^{\mathrm{B}}$

nisi spiritus

hominis AMst $\left.{ }^{\mathrm{var}} \mathrm{PEL}^{\mathrm{var}} \mathrm{SPM}^{\mathrm{var}}\right]$ om. 61 76* 777889 AMst $^{\mathrm{ed}} \mathrm{PEL}^{\mathrm{AB}}$ SPM $^{\text {ed }}$, qui in homine $(75) 7^{\mathrm{C} *}$

qui in ipso est ] qui $76^{*}$, qui in ipso 77

ita et $\mathrm{AMst}^{\mathrm{AD}}$ ] sic et $61647778 \mathrm{AMst}^{\mathrm{ed}} \mathrm{PEL}^{\mathrm{B}}$

quae 515458617688 ] om. 77

dei $\mathrm{AMst}^{\mathrm{R}} \mathrm{SPM}^{\mathrm{var}}$ ] in deo $617789 \mathrm{AMst}^{\mathrm{ed}} \mathrm{PEL}^{\mathrm{B}} \mathrm{SPM}^{\mathrm{ed}}$

sunt ] om. 757677

nemo ] om. $\mathrm{PEL}^{\mathrm{B}}$

cognouit ] scit $64 \mathrm{SPM}^{\mathrm{var}}$, nouit AMst ${ }^{\mathrm{var}}$

nisi ] si 54 *

spiritus dei ] spiritu huius dei SPMvar

2:12 Present: 51545861647576777888 89 AMst PEL spm

nos autem non ] nos non autem $\mathrm{AMst}^{\mathrm{R}}$

spiritum ] spiritu 64

mundi PEL ${ }^{\text {var }}$ ] huius mundi $5^{1} 54^{\mathrm{C}} 5^{8} 61\langle 64\rangle 75768889$ AMst PEL ${ }^{\mathrm{AB}}$ SPM, mundi huius 7778

accepimus ] accipimus 61

sed ] om. 88*

spiritum qui ex deo est ] spiritum dei 7576

ut sciamus quae a deo donata sunt 515458617688

nobis ] om. AMst var

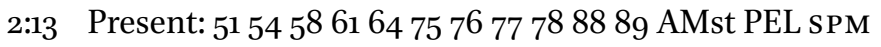

quae et loquimur 5154586176 ] quae etiam loquimur SPM

non PELvar $]$ om. PEL ${ }^{\mathrm{B}}$ 
in doctis AMstrar ${ }^{\mathrm{var}}$ docti 61, in sapientia 64, in doctrina 7576777889 AMsted, in suasoriis AMst ${ }^{\mathrm{R}}$, doctis $\mathrm{PEL}^{\mathrm{var}} \mathrm{SPM}^{\mathrm{var}}$, om. $\mathrm{PEL}^{\mathrm{B}}$, quae docentur $\mathrm{SPM}^{\mathrm{ed}}$ humanae sapientiae uerbis $5154587788^{C} \mathrm{AMst}^{\mathrm{var}}$ ] humanae doctis uerbis 64 , humana sapientiae uerbis 7576 , humanae sapientiae uerb $[1-2]\left(88^{*}\right)$, humanae sapientiae uerbi 89, uerborum humanae sapientiae AMst ${ }^{\text {ed }}$, per humanae sapientiae uerbum $\mathrm{SPM}^{\text {ed }}$, sapientiae humanae uerbis $\mathrm{SPM}^{\mathrm{var}}$ sed ] et PEL var

in doctrina $\left(88^{*}\right)$ ] doctrina $54 \mathrm{SPM}^{\mathrm{var}}$, docti 6164 , in ostensione $\mathrm{AMst}^{\mathrm{R}}$, doctis $\mathrm{PEL}^{\mathrm{B}}$, quae docentur $\mathrm{SPM}^{\mathrm{ed}}$

spiritus SPM ${ }^{\text {var }}$ ] spiritus sancti 58 , spiritu $6164 \mathrm{PEL}^{\mathrm{B}}$, per spiritum SPM${ }^{\mathrm{ed}}$; + sed $54,+$ et uirtute dei $\mathrm{AMst}^{\mathrm{R}}$

spiritalibus spiritalia 57

conparantes 5158757889 ] + uel conparamus 77

2:14 Present: 5154586164757677788889 AMst $^{\dagger}$ PEL SPM

animalis autem homo ] animalis homo $\mathrm{AMst}^{\mathrm{AD}}$, animalis enim homo $\mathrm{AMst}^{\mathrm{R}}$ non percipit ] non percipis $76^{*}$, nescit $\mathrm{AMst}^{\mathrm{R}}$

ea quae ${ }_{51} 545^{8} 61 \mathrm{AMst}^{\mathrm{var}} \mathrm{SPM}^{\mathrm{var}}$ ] quae $6475767789 \mathrm{AMst}^{\mathrm{ed}} \mathrm{SPM}^{\mathrm{ed}}$ sunt spiritus dei SPM $\left.{ }^{\text {var }}\right]$ spiritus dei sunt $61 \mathrm{SPM}^{\text {ed }}$, spiritus dei 7576 stultitia est enim illi 51 ] stultitia enim est illi 545864757689 AMst $^{\text {ted }}$ PEL $^{\text {var }}$, stultitia enim illi est 617778 , stultitia est enim illis $88^{*}$, stultitia autem illi est AMst $^{\mathrm{AD}}$, et stultitia est illi AMst ${ }^{\mathrm{R}}$, est enim stultitia illi AMst ${ }^{\mathrm{var}}$, stultitia enim illis est $\mathrm{PEL}^{\mathrm{B}}$

et non potest ] et non potes 76

intellegere $515488^{c} \mathrm{AMst}^{\mathrm{AD}}$ ] scire $61647576777889 \mathrm{AMst}^{\text {ed }} \mathrm{PEL}^{\mathrm{B}}$ SPM quia $\mathrm{AMst}^{\mathrm{D}} \mathrm{SPM}^{\text {var }}$ ] ea quae 54 , quoniam $647576777889 \mathrm{AMst}^{\mathrm{ed}} \mathrm{PEL}^{\mathrm{B}}$, quae $\mathrm{AMst}^{\mathrm{A}}$, quod $\mathrm{AMst}^{\mathrm{var}} \mathrm{SPM}^{\mathrm{ed}}$

spiritaliter 51

examinatur ] diiudicatur $616477 \mathrm{AMst}^{\mathrm{AD}} \mathrm{PEL}^{\mathrm{B}}$, iudicatur $75767889 \mathrm{AMst}^{\mathrm{ed}}$ $\mathrm{SPM}^{\mathrm{var}}$, interrogatur SPM ${ }^{\mathrm{ed}}$

2:15 Present: 5154586164757677788889 AMst PEL SPM

spiritalis autem $5^{1}$ ] qui spiritalis est autem $\mathrm{AMst}^{\mathrm{AD}}$, spiritalis uero $\mathrm{AMst}^{\mathrm{R}}$, qui autem spiritalis est SPM

iudicat omnia AMstrar SPM $^{\text {var }}$ ] diiudicat omnia 5861647778 AMst $^{\text {ed }}$ PEL $^{\mathrm{B}}$, inquirit omnia $\mathrm{SPM}^{\mathrm{ed}}$

et ipse $\left.\mathrm{AMst}^{\mathrm{R}} \mathrm{PEL}^{\text {var }}\right]$ ipse autem $61647576777889 \mathrm{AMst}^{\text {ed }} \mathrm{PEL}^{\mathrm{AB}} \mathrm{SPM}$

$\dagger \quad$ 1Cor. 2:14. Numerous manuscripts of AMst omit the second half of this verse. 
a nemine $\left(88^{*}\right)$

iudicatur $\left.\mathrm{AMst}^{\mathrm{var}}\right]$ diiudicatur $61\langle 64\rangle \mathrm{AMst}^{\mathrm{ed}} \mathrm{PEL}^{\mathrm{B}}$

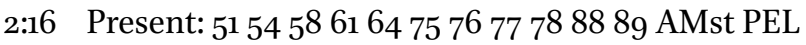

\pm scriptum est enim de esaia propheta $61^{*}$, scriptum est enim in esaia propheta $6{ }_{1} c$

quis enim cognouit ] quis enim intellegit 64

sensum domini ] + nisi spiritus domini $545^{8}$

qui ] quis $5^{1} \mathrm{AMst}^{\text {var }}$

instruat ] instruit $54^{\mathrm{C}} 58$, instruxit $\mathrm{AMst}^{\mathrm{var}}$

eum ] in eum $54^{*}$, illum $64 \mathrm{AMst}^{\mathrm{AD}}$

nos autem sensum ] nos autem intellectum 64

christi habemus AMst ${ }^{A D R}$ PEL $^{\text {var }}$ ] domini habemus 6164777889 AMst $^{\text {ed }}$ $\mathrm{PEL}^{\mathrm{AB}}$

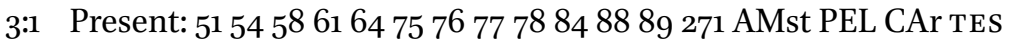
et ego $\left(64^{*}\right)$ ] ego $88^{*}$ AMst $^{\text {var }}$, et ego quidem TES ${ }^{\text {ed }}$, ego quidem TES ${ }^{\text {var }}$, abs. 84271

fratres non potui uobis loqui ] fratres non potui loqui uobis 5464757677 $78 \mathrm{PEL}^{\text {var }}$ CAr, uobis fratres non potui loqui 61

quasi ] tamquam 51 TES $^{\text {var }}$, abs. 84 spiritalibus sed quasi carnalibus 88 ] abs. 84

tamquam AMst ${ }^{\mathrm{AD}}$ ] quasi 61647576777889 AMst $^{\mathrm{ed}} \mathrm{PEL}^{\mathrm{B}}$ TES, abs. 84 CAr

paruulis $75 * 89 P E L^{A}$ TES $^{\text {var }}$ ] paruulus $\mathrm{PEL}^{\mathrm{B} *}$, infantibus TES ${ }^{\mathrm{ed}}$, abs. $84 \mathrm{CAr}$ in christo ] abs. $84 \mathrm{CAr}$

3:2 Present: $5154586164757677788889271^{\dagger}$ AMst PEL TES

lac uobis Te $S^{\text {var }}$ ] lac uos uel uobis 77 , lac uo[1-2]bis $88^{*}$, lacte uos TES $S^{\text {ed }}$ potum dedi TES ${ }^{\text {var }}$ ] potauit uel potum dedi 77 , potaui TES ${ }^{\text {ed }}$

non ] et non $75767778 \mathrm{AMst}^{\mathrm{D}}$

escam 61 TES $^{\text {var }}$ ] esca 64 , cibo TES $S^{\text {ed }}$

nondum enim ] abs. 271, dum enim adhuc pusilli eratis et TES ${ }^{\text {var }}$, necdum enim TES ${ }^{\text {var }}$

poteratis sed ] poteratis $88^{*}$ TES $^{\text {var }}$, abs. 271

ne ] nec $5^{1} 545^{8} 616488^{\mathrm{C}} \mathrm{AMst}^{\mathrm{AD}}$ PEL TES ${ }^{\mathrm{var}}$, neque $75767778 \mathrm{AMst}^{\text {ed }}$ TES ${ }^{\text {ed }}$, donec $88^{*}$, nec usque 89 , abs. 271

$\dagger \quad$ 1Cor. 3:2. After the word escam in this verse, the lection in VL 271 jumps to 3:7. 
nunc ] adhuc $616475767889 \mathrm{AMst}^{\mathrm{AD}} \mathrm{PEL}^{\mathrm{B}}$, adhuc nunc 77 , abs. 271 quidem AMst ${ }^{\mathrm{AD}}$ TES ${ }^{\text {var }}$ ] om. 6175767789 AMst $^{\text {ed }} \mathrm{PEL}^{\mathrm{B}} \mathrm{TES}^{\mathrm{ed}}$, abs. 271 potestis adhuc enim 54 ] abs. 271, potestis adhuc autem $\mathrm{AMst} \mathrm{AD}^{\mathrm{AD}}$ estis carnales $88^{*}$ ] abs. 271, carnales estis AMstvar

3:3 Present: 5154586164757677788889 AMst PEL tes SPM SPE cum enim AMst ${ }^{\mathrm{var}} \mathrm{TES}^{\mathrm{var}}$ ] nam cum $61 \mathrm{AMst}^{\mathrm{ed}} P E L^{B}$, cum autem 64, ubi enim TES ${ }^{\text {ed }}$

sit ] om. $5^{8} 77 \mathrm{AMst}^{\mathrm{ed}} \mathrm{TES}^{\text {ed }}$, sint $616489 \mathrm{AMst}^{\mathrm{AD}} \mathrm{PEL}^{\mathrm{B}} \mathrm{SPM}^{\mathrm{var}}$, lac. $88^{*}$, est TES $^{\text {var }}$, sunt TES ${ }^{\text {var }}$

inter uos $\left.\mathrm{AMst}^{\mathrm{AD}} \mathrm{SPE}^{\mathrm{var}}\right]$ in uobis AMst ${ }^{\mathrm{ed}} \mathrm{TES} \mathrm{SPE}^{\mathrm{ed}}$; + sit 5877

zelus $\mathrm{SPM}^{\mathrm{var}}$ ] aemulationes $6189 A M s t^{A D} P E L^{B} \mathrm{SPM}^{\mathrm{var}}$, aemulatio 647576 $77 \mathrm{AMst}^{\mathrm{ed}} \mathrm{TES}^{\mathrm{ed}} \mathrm{SPM}^{\mathrm{ed}}$, dissimulatio TES ${ }^{\mathrm{var}}$

et contentio $5458 \mathrm{SPM}^{\mathrm{var}}$ ] et contentiones $6189 \mathrm{AMst}^{\mathrm{ed}} \mathrm{PEL}^{\mathrm{B}} \mathrm{SPM}^{\mathrm{ed}}$, om. $\mathrm{AMst}^{\mathrm{AD}}$, et dissensio $\mathrm{TES}^{\mathrm{var}}$

\pm et dissensiones 617576777889 PEL ${ }^{B}$ PEL $^{\text {var }}$ TES $^{\text {ed, }}$, sint AMst ${ }^{\text {ed }}$, sunt TES ${ }^{\text {var }}$

nonne carnales estis $7588^{*}$

et ] sed $\mathrm{SPE}^{\mathrm{var}}$

secundum hominem $88^{C}$

ambulatis ] ambulastis 89 , ambulans $\mathrm{PEL}^{\mathrm{B}}$

3:4 Present: 51545861647576777888 89 AMst PEL SPE cum enim ] quis enim 58 , non enim $88^{*}$

quis dicit ] quis dicat $5^{1} 5^{8} 6164$, dicit quis AMstvar, dicat quis $\mathrm{AMst}^{\mathrm{AD}} \mathrm{PEL}^{\mathrm{B}}$ ego ] e[r] go $88^{*}$

quidem sum pauli $\mathrm{AMst}{ }^{\mathrm{AD}}$ ] sum pauli $6189 \mathrm{AMst}^{\mathrm{ed}} \mathrm{PEL}^{\mathrm{B}}$, quidem pauli $S P E^{\text {var }}$, sum quidem pauli SPE ${ }^{\text {var }}$

alius autem ] alius uero 89 ; + et $P E L^{B}$

ego apollo $6 r$ ] apollo $89 \mathrm{SPE}$ var

nonne homines estis $88^{*}$ ] nonne homines PEL var

3:5 Present: 51545861647576777888 89 AMst PEL

quid ] quis 7778

igitur est ] est ergo 61, ergo est 647778

apollo 61 ] apostolus $\mathrm{AMst}^{\mathrm{A}}$

quid 54 ] quis 7778 ; + est $\mathrm{PEL}^{\mathrm{B}}$

uero ] autem 647576 , etiam $89 \mathrm{AMst}^{\mathrm{AD}}$

paulus ministri ] paulus minister $5^{8}$

eius cui ] per quos $64 \mathrm{AMst}^{\mathrm{AD}}$ 
credidistis et unicuique sicut $54\langle 64\rangle 88^{*}$

dominus dedit ] deus dedit $58 \mathrm{AMst}^{\mathrm{var}}$

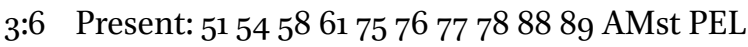

ego plantaui apollo 61

rigauit ] inrigauit $757689 \mathrm{PEL}^{\mathrm{A}}$

sed deus ] sed dominus 61, deus PEL ${ }^{B}$

incrementum dedit

3:7 Present: 51545861757677788889 271 AMst PEL

itaque

neque qui plantat ] qui plantat neque 61, qui plantat $\mathrm{AMst} \mathrm{AD}^{\mathrm{AD}}$

est aliquid 54 ] est quicquam $61 A M s t^{R}$

neque qui rigat $\langle 76\rangle_{271^{*}}$ ] neque qui inrigat $89 \mathrm{AMst}^{\mathrm{AD}} \mathrm{PEL}^{\mathrm{A}}$

sed qui 88 ] sed $\mathrm{AMst}^{\mathrm{D} *}$

incrementum dat deus $(78)$ ] dat incrementum deus $61 \mathrm{PEL}^{\mathrm{B}}$; + ipsi gloria in saecula saeculorum amen $271^{\dagger}$

3:8 Present: $515458617576\langle 77\rangle^{\dagger} 788889$ AMst PEL

qui plantat autem ] qui autem plantat $5861 \mathrm{AMst}^{\mathrm{ed}} \mathrm{PEL}^{\mathrm{B}}$, qui plantat enim $\mathrm{AMst}^{\mathrm{AD}}$, qui enim plantat $\mathrm{AMst}^{\mathrm{R}}$

et qui rigat ] et qui rigant $\mathrm{AMst}^{\mathrm{var}}$, et qui inrigat $\mathrm{PEL}^{\mathrm{A}}$

unum sunt unusquisque

autem ] om. $54^{*}$, enim PEL var

propriam ] suam $\mathrm{PEL}^{\mathrm{B}}$

mercedem accipiet ] + a domino 61

secundum suum laborem $\left(88^{*}\right)$

3:9 Present: $51545^{8} 617576\langle 77\rangle 788889251$ AMst PEL

\pm fratres 251

dei enim sumus ] dei sumus PEL $\mathrm{A}^{\mathrm{A}}$ + operis $\mathrm{AMst}^{\mathrm{ed}}$

adiutores $88 \mathrm{AMst}^{\mathrm{ADR}}$ ] cultores $88^{\text {alt }}$, participes $\mathrm{AMst}^{\text {ed }}$

dei ] om. $88^{*}$

agricultura $78 \mathrm{AMst}^{\mathrm{AD}}$ ] cultura $7576 \mathrm{AMst}^{\text {ed }} \mathrm{PEL}^{\mathrm{B}}$

estis ] om. 7576 AMst, est 89 PEL $^{\text {B }}$

$\dagger \quad 1$ Cor. 3:7. This is a standard conclusion to a lection in VL 271 .

$\dagger{ }_{1}$ Cor. $3: 8-16$. There is a blank space in VL 77 where these verses should be, implying that they were present in the Latin exemplar. 
dei aedificatio 51545876

estis AMstvar ${ }^{\text {var }}$ est $89 \mathrm{AMst}^{\text {ed }} \mathrm{PEL}^{\mathrm{B}}$

3:10 Present: $51545^{8} 617576\langle 77\rangle 788889$ 251 AMst PEL secundum gratiam 58251

dei AMst' ${ }^{\text {var }}$ ] om. 51587889 AMst $^{\text {ed }}$ PEL $^{\text {var }}$

quae data est mihi 515458617688 ] quae data est $\mathrm{AMst}{ }^{\mathrm{AD} *}$

ut sapiens architectus fundamentum

posui ] posuit $\mathrm{AMst}^{\mathrm{R}}$

alius ] alter $757689 \mathrm{AMst}^{\mathrm{AD}} \mathrm{PEL}^{\mathrm{B}}$

autem AMst ${ }^{\text {var }}$ PEL $^{\text {var }}$ ] om. 757689 AMsted $^{\text {ed }}$ PLAB $^{\mathrm{AB}}$

superaedificat unusquisque autem uideat 5154587688

quomodo $\mathrm{AMst}^{\mathrm{AD}}$ ] quemadmodum AMsted

superaedificet $51545888 \mathrm{AMst}^{\mathrm{var}}$ ] superaedificat $61757625_{1} \mathrm{AMst}^{\mathrm{ed}}$

3:11 Present: $515458617576\langle 77\rangle 788889251$ AMst PEL

fundamentum ] fundamenta $88^{*}$

enim ] autem $P E L^{B}$

aliud nemo potest ponere praeter id quod positum est 51545861767888 251

qui est $\mathrm{AMst} \mathrm{AD}^{\mathrm{AD}}$ ] quod est $514^{\mathrm{C}} 5^{8} 6178 \mathrm{AMst}^{\text {ed }} \mathrm{PEL}^{\mathrm{B}}, \mathrm{q}\left[{ }^{1-2}\right]$ est $54^{*}$

christus iesus ] iesus christus 61, christus $\mathrm{PEL}^{\mathrm{B}}$

3:12 Present: $51545^{8} 61757^{6}\langle 77\rangle 7^{8} 8889$ 251 AMst PEL

si quis autem $\left(88^{*}\right)$

superaedificat $5^{1} 545^{8} 7688$ ] superaedificauerit $7_{8} P E L^{B}$, superaedificet AMst $^{\text {var }}$

supra fundamentum ] super fundamentum $757689 \mathrm{AMst}^{\text {var }} \mathrm{PEL}^{\text {var }}$

hoc aurum ] istud aurum PEL ${ }^{\mathrm{A}}$; + et PEL ${ }^{\mathrm{B}}$

argentum lapides pretiosos 5154586175 (78) 8889251

ligna ] lignum AMst ${ }^{\mathrm{var}}$

faenum 515458617576788889

stipulam ] stipula 89 AMstrar $^{\mathrm{va}}$

3:13 Present: $51545861757^{6}\langle 77\rangle 7^{8} 8889251$ AMst PEL

uniuscuiusque $\left(7^{*}\right) \mathrm{AMst}^{\mathrm{AD}} \mathrm{PEL}^{\mathrm{var}}$ ] qui fecerit hoc $6189 \mathrm{PEL}^{\mathrm{B}}$, qui fecit hoc 7576 , qui facit hoc AMsted, cuiusque $\mathrm{AMst}^{\mathrm{R}}$; + autem $\mathrm{PEL}^{\mathrm{A}}$

opus manifestum erit $\mathrm{AMst}^{\mathrm{ADR}}$ ] opus manifestus erit $61757689 \mathrm{AMst}^{\mathrm{ed}}$ PEL $^{\mathrm{B}}$

dies enim ] om. AMstvar 
\pm domini $5158617888251 \mathrm{AMst}^{\mathrm{AD}} \mathrm{PEL}^{\mathrm{B}}$, dei iudicii PEL ${ }^{\mathrm{A} *}$, iudicii PEL ${ }^{\mathrm{AC}}$

declarabit PEL ${ }^{\mathrm{AC}}$ ] declarauit $54251 \mathrm{PEL}^{\mathrm{A} *}$, manifestabit $6176 \mathrm{PEL}^{\mathrm{B}}$, manifestauit 7589 , om. AMstrar; + eum 61 quia ] qui 51, quoniam $617576 \mathrm{PEL}^{\mathrm{B}}$, que $\mathrm{AMst}^{\mathrm{A}}{ }^{*}$ in igne reuelabitur et uniuscuiusque opus quale sit ] quale opus sit $\mathrm{PEL}^{\mathrm{B}}$ ignis probabit ] ignis probauit 7589

3:14 Present: $51545^{8} 61757^{6}\langle 77\rangle 7^{8} 8889$ 251 AMst PEL

si ] sed 51

cuius opus manserit quod ] cuiusque opus arserit quod $P E L^{B}$

superaedificauit $51545^{8} 88 \mathrm{AMst}^{\mathrm{var}}$ ] superaedificauerit $61757689 \mathrm{AMst}^{\mathrm{ed}}$ $P E L^{B}$

mercedem accipiet

3:15 Present: $515458617576\langle 77\rangle 788889$ 251 AMst PEL

\pm et 61

si cuius opus arserit

detrimentum (88) $\mathrm{AMst}^{\mathrm{var}}$ ] damnum AMsted, iacturam PEL ${ }^{\mathrm{B}}$

patietur ipse autem 5154 ] patietur ipse tamen $\mathrm{AMst}^{\mathrm{var}}$

saluus erit sic tamen quasi per ignem

3:16 Present: 5154586175767778848889 251 AMst PEL CAr TES SPM SPE nescitis quia ] fratres nescitis quia $84, a b s .77$

templum dei estis $\langle 84\rangle]$ dei templum usque hoc estis TES ${ }^{\mathrm{var}}$, abs. 77

et spiritus dei ] abs. 7784

habitat in uobis ] habitet in uobis AMst ${ }^{\mathrm{var}} \mathrm{SPE}^{\mathrm{var}}$, in uobis inhabitat TES ${ }^{\mathrm{var}}$, abs. 84

3:17 Present: 51545861757677788889 251 AMst PEL TES SPM SPE si quis TES $\left.{ }^{\mathrm{var}}\right]$ si qui TES $\mathrm{S}^{\mathrm{ed}}$

autem AMstvar ${ }^{\mathrm{va}}$ om. $5^{1} 757^{6} 777889$ AMst $^{\text {ed }}$ PEL $^{\mathrm{B}}$ TES

templum dei uiolauerit ] dei templum uiolauerit $\mathrm{TES}^{\mathrm{var}}$, uiolauerit templum dei TES ${ }^{\text {var }}$

disperdet $\mathrm{TES}^{\mathrm{var}}$ ] disperdit $5488 \mathrm{PEL}^{\mathrm{var}}$, uiolabit TES $\mathrm{S}^{\mathrm{ed}} \mathrm{SPM}$, uiolauit TES $\mathrm{S}^{\mathrm{var}}$ illum deus ] eum deus $\mathrm{PEL}^{\mathrm{B}}$

templum enim dei ] templum enim $61^{*} 76 \mathrm{SPE}^{\mathrm{var}}$, abs. TES

sanctum est ] non sanctum est $54^{*}$, abs. TES

quod estis uos ] quod estis $\mathrm{SPE}^{\mathrm{var}}$, abs. TES 
3:18 Present: 51545861757677788889 AMst PEL tes SPM SPE

nemo se ] nemo uos 5458617576777889 PEL $^{\mathrm{B}}$, nemo 88* PEL ${ }^{\mathrm{var}}$, nemo seipsum $\mathrm{AMst}^{\mathrm{AD}}$

seducat $\mathrm{SPM}^{\mathrm{var}}$ ] fallat AMst ${ }^{\mathrm{AD}} \mathrm{SPM}^{\mathrm{ed}}$, decipiat TES; + uanis uerbis 7576

si quis TES ${ }^{\mathrm{var}}$ ] si qui TES $\mathrm{S}^{\text {ed }}$

uidetur inter uos sapiens esse $\mathrm{SPM}^{\mathrm{var}}$ ] inter uos uidetur sapiens esse $5^{1}$, uidetur sapiens esse inter uos 6175767778 AMst PEL ${ }^{\mathrm{B}}$, uidetur sapiens inter uos 89 , putat sapientem se esse in uobis TES ${ }^{\text {ed }}$, se putat sapientem esse in uobis TES ${ }^{\mathrm{var}}$, putat se sapientem esse in uobis $\mathrm{TES}^{\mathrm{var}}$, uidetur sapiens esse $\mathrm{SPM}^{\text {ed }}$

in hoc saeculo 515458617688 ] in saeculo hoc $77\left(7^{*}\right)^{C *}$, saeculo huic TES $S^{\text {ed }}$, mundo hoc $\mathrm{TES}$ var

stultus fiat ] stultus fiet 88

ut sit ] ut si $89^{*}$, ut fiat TES ${ }^{\text {ed }}$, et sit TES ${ }^{\text {var }}$

sapiens ] + apud deum $61 \mathrm{TES}^{\mathrm{var}}$

3:19 Present: 515458617576777888 89 AMst PEL TES SPM SPE

sapientia enim 545888 ] nam sapientia $89 \mathrm{SPM}$, sapientia TES $\mathrm{S}^{\mathrm{var}}$

huius mundi ] huius saeculi 617576 , mundi huius $7778 \mathrm{TES} \mathrm{S}^{\mathrm{var}}$

stultitia est apud deum $51545^{8} 7588$ ] stultitia apud deum est 77

scriptum est enim ] scriptum enim TE $\mathrm{S}^{\mathrm{var}}$, abs. SPE

conprehendam $515878\left(88^{*}\right)$ ] adprehendam $617576 A M s t^{R} P E L^{B}$, adprehendam uel adprehendit 77 , adprehendens $89 \mathrm{TES}^{\mathrm{var}}$, reprehendens AMsted $\mathrm{TES}^{\mathrm{ed}}$, conprehendens $\mathrm{AMst}^{\mathrm{AD} *} \mathrm{TES}^{\mathrm{var}}$, conprehendes $\mathrm{TES}^{\mathrm{var}} \mathrm{SPM}^{\mathrm{var}}$, reprehendes TES ${ }^{\mathrm{var}}$, reprehendam TES ${ }^{\mathrm{var}}$, abs. SPE

sapientes in ] abs. SPE

astutia 545888 ] astutiam PEL ${ }^{\mathrm{var}}$, astutis TES ${ }^{\mathrm{var}}$, abs. SPE

eorum $\mathrm{AMst}^{\mathrm{var}} \mathrm{TES}^{\mathrm{var}}$ ] ipsorum $\mathrm{AMst}^{\mathrm{ed}} \mathrm{TES}^{\mathrm{ed}}$, abs. SPE

3:20 Present: 51545861757677788889 AMst PEL TES SPM

et iterum ] item illic TES ${ }^{\text {var }}$

dominus nouit ] nouit dominus PEL ${ }^{\text {var }}$, dominus cognouit TES ${ }^{\text {ed }}$, cognoscet deus TESvar

cogitationes sapientium $\left.545^{8}\left(89^{*}\right)\right]$ cogitationes hominum 61 PEL ${ }^{\text {var }}$ quoniam TES $^{\text {var }}$ ] quam $\mathrm{PEL}^{\mathrm{B}}$, quia TES ${ }^{\text {ed }}$

uanae sunt $\left.51545^{8} 767^{8} 88 \mathrm{TES}^{\text {var }}\right]$ sunt uanae $\left(77^{*}\right)^{\mathrm{C*}} 78^{\mathrm{gl}}$, sunt stultae TES $S^{\text {ed }}$, stultae sunt TES ${ }^{\text {var }}$

$\dagger$ 1Cor. 3:20. The variant in TES misinterprets the Pauline text as the introduction to the extract. 
3:21 Present: 51545861757677788889 AMst PEL

itaque nemo glorietur in

hominibus $\left(54^{*}\right) \mathrm{AMst}^{\mathrm{AD}}$ ] homine $61777889 \mathrm{AMst}^{\mathrm{ed}} \mathrm{PEL}^{\mathrm{B}}$, homines 7576 omnia enim ] nam omnia 89, omnia $\mathrm{AMst}^{\mathrm{AD}}$, omnia namque $\mathrm{AMst}^{\mathrm{R}}$ uestra sunt

3:22 Present: 515458617576777888 89 AMst PEL siue paulus $P E L^{\text {var }}$ ] om. PEL $^{\mathrm{B}}$ siue apollo siue cephas 88 ] siue cephas siue apollo $6 r$ siue mundus ] siue hic mundus $75\langle 76\rangle 89 \mathrm{PEL}^{\mathrm{var}}$ siue uita siue mors siue praesentia siue futura omnia 5154586176777888 89

enim ] autem $5^{1} 88^{*}$, om. 61757689 AMst PEL $^{\mathrm{B}}$ uestra sunt $\mathrm{AMst}^{\mathrm{AD}}$ ] uestra $757689 \mathrm{AMst}^{\mathrm{ed}}$

3:23 Present: 515458617576777888 89 AMst PEL uos autem christi christus autem dei ] christus uero dei AMst ${ }^{\mathrm{ADR}}$

4:1 Present: $51545^{8} 617576777888$ 89 AMst PEL sic $\mathrm{AMst}^{\mathrm{AD}}$ ] sic enim 61, ita $\mathrm{AMst}^{\text {ed }} \mathrm{PEL}^{\mathrm{B}}$ nos existimet ] nos aestimet AMst ${ }^{\text {var }}$ homo ut ministros christi et dispensatores mysteriorum 586176 ] ministeriorum $5488^{*} \mathrm{PEL}^{\text {var }}$ dei ] om. 89

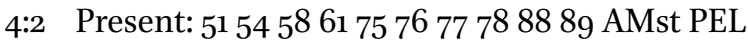
hic iam ] + uel ceterum 77 quaeritur inter dispensatores ut 5154587677 (88) fidelis quis inueniatur ] quis fidelis inueniatur 7778

4:3 Present: 515458617576777888 89 AMst PEL mihi autem $515458 \mathrm{AMst}^{\mathrm{AD}}$ ] mihi enim $\mathrm{AMst}^{\mathrm{ed}}$, mihi $\mathrm{AMst}^{\mathrm{var}}$ pro minimo est ] in minimum est 77 ut a uobis $(77)$ iudicer ] diiudicer $\mathrm{AMst}^{\mathrm{var}}$, iudicetur $\mathrm{PEL}^{\mathrm{B}}$ aut ab humano die 5488 ] aut ab humana die $77 \mathrm{AMst}^{\mathrm{var}}$ sed neque me ipsum iudico ] diiudico $\mathrm{AMst}{ }^{\mathrm{AD}}$ 


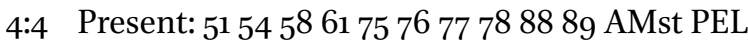

nihil enim 515458 ] nihil quidem AMst $^{\mathrm{R}}$

mihi conscius sum 5154 ] mihi conscius 58 , sum mihi conscius 61 , mihi ipsi sum conscius 77

sed non in hoc iustificatus ] om. 58, sed non hoc iustificatus 61 sum qui autem

iudicat me ] me iudicat $61 \mathrm{PEL}^{\text {var }}$, diiudicat me $\mathrm{AMst}^{\mathrm{A}} P E L^{\text {var }}$, iudicat $P E L^{\mathrm{var}}$ dominus est ] dominus 89 ; + deus 75

4:5 Present: 51545861757677788889 AMst PEL SPM SPE

itaque ] ideoque SPM

nolite ] ne 77, noli AMstrar

ante tempus iudicare ] ante tempus aliquid iudicare $6178 \mathrm{SPM}^{\text {ed }}$, ante tem-

pus aliquid iudicate 77 , ante tempus quid iudicare $\mathrm{AMst}^{\mathrm{AD}} \mathrm{PEL}^{\mathrm{A}}$, iudicare ante tempus PEL ${ }^{\mathrm{var}}$, ante tempus quicquam iudicare $\mathrm{SPM}^{\mathrm{var}}$

quoadusque ] quousque $58 \mathrm{SPM}^{\mathrm{ed}}$, donec $61777889 \mathrm{AMst}^{\mathrm{ed}} \mathrm{PEL}^{\mathrm{B}} \mathrm{SPM}^{\mathrm{var}}$, usque cum 7576 , usquedum $\mathrm{AMst}^{\mathrm{AD}}$

ueniat dominus $(78)\left(88^{*}\right)$ ] uenit dominus $757^{6}$

qui ] om. $75767778 \mathrm{AMst}^{\mathrm{AD}}$

et $\mathrm{AMst}^{\mathrm{ADR}} \mathrm{PEL}^{\mathrm{var}} \mathrm{SPM}^{\mathrm{var}}$ ] om. $61 \mathrm{AMst}^{\mathrm{ed}} \mathrm{PEL}^{\mathrm{AB}} \mathrm{SPM}^{\mathrm{ed}} \mathrm{SPE}^{\mathrm{var}}$

inluminabit $515^{8}$ ] inluminauit $547589 \mathrm{SPE}^{\mathrm{var}}$, inluminet $7778 \mathrm{SPM}^{\mathrm{var}}$; + quae sunt $77 \mathrm{AMst}^{\mathrm{AD}}$

abscondita (88) $\mathrm{AMst}^{\mathrm{var}}$ ] occulta $61757689 \mathrm{AMst}^{\mathrm{ed}} \mathrm{PEL}^{\mathrm{B}} \mathrm{SPM}^{\mathrm{var}}$, absconsa $\mathrm{AMst}^{\mathrm{AD}} \mathrm{PEL}^{\mathrm{var}}$, om. SPM $^{\text {ed }}$

tenebrarum et $\left.\mathrm{SPM}^{\mathrm{var}}\right] \mathrm{om}$. $\mathrm{SPM}^{\mathrm{ed}}$

manifestabit ] manifestauit $547589 \mathrm{SPE}^{\mathrm{var}}$, manifestet $\mathrm{SPM}^{\mathrm{var}}$, om. SPM $\mathrm{M}^{\mathrm{ed}}$

consilia ] [2] consilia PELA ${ }^{\mathrm{A}}$, cogitationes $\mathrm{SPM}^{\mathrm{var}}$

cordium ] cordis $\mathrm{AMst}^{\mathrm{AD}} \mathrm{SPM}$

et tunc laus erit unicuique a deo $\langle 54\rangle$

4:6 Present: 51545861757677788889 AMst PEL SPE

haec autem fratres 51545876 (88)

transfiguraui ] figuraui AMstvar, transfiguram $\mathrm{PEL}^{\mathrm{var}}$

in me ] + ipsum 7778

et apollo propter uos $61\left(88^{*}\right)$ ] et apostolo propter uos PEL var

ut in ] ut a $88^{\mathrm{C}}$

nobis ] uobis $58^{*} 75^{\mathrm{C}} 76 \mathrm{SPE} \mathrm{Ear}^{\mathrm{var}}$

discatis ne supra ] discatis ne super $\mathrm{AMst}^{\mathrm{AD}}$

quam scriptum AMst ${ }^{\text {var }}$ ] quod scriptum $58617778 \mathrm{AMst}^{\text {ed }} \mathrm{PEL}^{\mathrm{var}}$

est $\left\langle{ }_{76} 6\right\rangle$ ] est sapere $\mathrm{AMst}^{\mathrm{A}}$, est supra $\mathrm{AMst}^{\mathrm{D}} ;+$ ut non $777^{8} \mathrm{AMst}^{\mathrm{var}}$ 
unus ] om. AMstrar

aduersus alterum $\mathrm{AMst}^{\mathrm{D}}$ ] aduersus alium 51, pro uno $61 \mathrm{AMst}^{\mathrm{ed}} \mathrm{PEL}^{\mathrm{B}}$, aduersus alterum uel per uno 77, aduersum alterum $\mathrm{AMst}^{\mathrm{A}}$

infletur

pro alio $\mathrm{AMs} \mathrm{t}^{\mathrm{AD}}$ ] aduersus me alterum $61^{*}$, aduersus alterum ${ }_{61}{ }^{\mathrm{C}} \mathrm{AMst}^{\mathrm{var}}$ $\mathrm{PEL}^{\mathrm{B}}$, aduersus alterum uel pro alio 77 , aduersum alterum $\mathrm{AMst}^{\mathrm{ed}}$

4:7 Present: 515458617576777888 89 AMst PEL TES SPE

quis enim ] nam quis $\mathrm{AMst}^{\mathrm{R}}$, quid enim TES

te discernit ] om. TES

quid $\left.54^{*}\right]$ om. TES, quod SPEvar

autem ] enim $\mathrm{AMst}^{\mathrm{R}}$, ait $\mathrm{PEL}^{\mathrm{B}}$, om. TES

habes 54 ] om. $76^{*}$

quod non accepisti 61 TES $^{\text {var }}$ ] quod non acceperis TES ${ }^{\text {ed }}$

si autem ] quod si $757689 \mathrm{AMst}^{\mathrm{AD}} \mathrm{PEL}^{\mathrm{A}}$ TES $^{\mathrm{var}}$, si autem et 77

accepisti 61

quid $54^{*}$ ] qui $54^{\mathrm{C}}$, quis AMstrar

gloriaris quasi non

acceperis ] acciperes 58 , acciperis 61 , accipis TES ${ }^{\mathrm{var}} ;+$ regnum TES ${ }^{\mathrm{var}}$

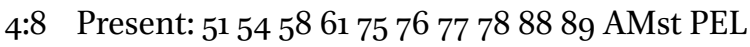

iam saturati estis $\left(77^{*}\right)$

iam $\mathrm{AMst}^{\mathrm{var}}$ ] om. $7576 \mathrm{AMst}^{\mathrm{ed}} \mathrm{PEL}^{\mathrm{B}}$

diuites facti estis ] + iam $5^{8},+$ uel ditati estis 77

sine nobis ] siue uobis $\mathrm{PEL}^{\mathrm{B}}$

regnastis ] regnatis $5^{1} 545^{8} 6176777888$ AMst PEL, om. 89

et utinam ] utinam $75^{*}$ om. 89

regnaretis AMstrar $]$ regnetis $5^{1} 5^{8} 6175767788 \mathrm{AMst}^{\text {ed }} \mathrm{PEL}^{\mathrm{A} *}$, regnatis 89

ut et nos uobiscum $(78)$ ] ut et nos nobiscum $89^{*}$

regnaremus $\mathrm{AMst}^{\mathrm{var}}$ ] regnemus $515^{8} 617^{\mathrm{C}} 768889 \mathrm{AMst}^{\text {ed }} \mathrm{PEL}^{\mathrm{A}}$, regnetis

$75^{*}$, corregnemus 77

4:9 Present: 51545861757677788889 271 AMst PEL CAr

puto enim ] fratres puto 271 ; quod $58 \mathrm{AMst}^{\mathrm{R}}$

deus nos apostolos nouissimos ostendit $\left.77^{*} 271^{*}\right]$ nos deus apostolos nouissimos ostendit $545889 \mathrm{PEL}^{\mathrm{B}} \mathrm{CAr}$, deus nos apostolos ostendit nouissimos 61 , nos deus nouissimos apostolos ostendit $\mathrm{AMst}^{\mathrm{var}}$, deus nouissimos apostolos nos ostendit PEL var

tamquam $77 \mathrm{AMst}^{\mathrm{AD}}$ ] quasi $61 \mathrm{AMst}^{\text {ed }} \mathrm{PEL}^{\mathrm{B}}$, abs. $\mathrm{CAr}$

morti destinatos $61\langle 76\rangle(271)]$ abs. CAr 
quia ] quoniam $6175767778 \mathrm{PEL}^{\mathrm{B}}$, abs. CAr

spectaculum $\left(78^{*}\right)$ ] exspectaculum 271 PEL ${ }^{\text {var }}$, abs. CAr

facti sumus ] sumus $88^{*}$, abs. CAr

mundo $\mathrm{AMst}^{\mathrm{R}}$ ] huic mundo $545^{8} 61757^{6} 7^{8} 88^{\mathrm{C}} 89 \mathrm{AMst}^{\mathrm{ed}} \mathrm{PEL}^{\mathrm{B}}$, abs. CAr et angelis et 61 ] abs. CAr

hominibus $271^{C}$ ] omnibus $271^{*}$, abs. CAr

4:10 Present: 51545861757677788889 271 AMst PEL

nos ] uos 271

stulti propter christum 88

uos ] nos 271

autem prudentes in christo

nos infirmi ] nos autem infirmi 61

uos autem fortes ] uos fortes $54 \mathrm{AMst}^{\mathrm{AD}}$

uos nobiles ] nos nobiles $89^{*}$, uos autem nobiles AMstvar

nos autem ] nos uero $77 \mathrm{AMst}^{\mathrm{var}}$

ignobiles ] inhonorati uel ignobiles 77

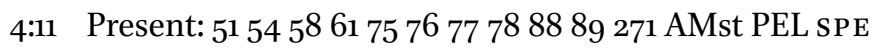

usque in $\mathrm{AMst}^{\mathrm{var}}$ ] usque ad $54 \mathrm{AMst}^{\mathrm{R}} \mathrm{PEL}^{\text {var }}$, usque AMst $\mathrm{Ad}^{\mathrm{ed}}$

hanc horam $75^{*}\left(88^{*}\right) 271$ ] horam hanc 58

et esurimus 61 ] et esuribimus 271

et sitimus et nudi sumus

et colaphis caedimur ${ }_{51}(54) 5888271 A M s t^{A D}$ ] et colaphizamur 61757677

78 AMst $^{e d} P E L^{B}$, et colaphis uapulamus 89 , om. AMst ${ }^{\mathrm{var}}$

et instabiles sumus ] et stabiles sumus 61, om. AMstvar

4:12 Present: 51545861757677788889 271 AMst PEL SPE

et laboramus (78) ] laboramus 89

operantes manibus nostris 271 ] operantes propriis manibus 7778 , manibus nostris operantes 89 AMstrar $^{\text {var }}$

maledicimur ] maledicimus 89 , om. $\mathrm{PEL}^{\mathrm{A}}$, maledicemur $\mathrm{PEL}^{\mathrm{var}}$

et benedicimus ] om. PELA, et benedicemus PEL var

persecutionem (54) 58 ] om. $\mathrm{PEL}^{\mathrm{A}}$

patimur ] patiamur 61, patiemur 75 , passi 77, om. $\mathrm{PEL}^{\mathrm{A}}$

et sustinemus ] om. PELA

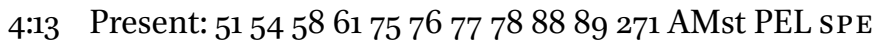

blasphemamur et 61271 AMsted ] blasphemati 7576 , blasphemati deprecamur et $\mathrm{AMst}^{\mathrm{AD}}$ 
obsecramus ] obsecramur 77 , obseruamus $\mathrm{PEL}^{\mathrm{B}}$

\pm persecutionem patimur et sustinemus PEL ${ }^{\mathrm{A}}$

tamquam purgamenta $75^{*} 77$ ] tamquam purgamentum $\mathrm{PEL}^{\mathrm{B}}$

huius ] om. $77271^{\mathrm{C}}$, lac. $271^{*}$

mundi facti sumus

omnium ] ab [5] omnium $271^{*}$, omni $271^{\mathrm{C}}$

peripsima $757677\left(P E L^{A *}\right)^{\mathrm{C}}$ ] purgamenta 61, purissima[1] 271*, inpurissima $271^{\mathrm{C}}$, lustramenta $\mathrm{AMst}^{\mathrm{R}}$, per infima $\mathrm{PEL}^{\mathrm{B}}$

usque adhuc 271 ] usque modo $\mathrm{AMst}^{\mathrm{R}}$

4:14 Present: 51545861757677788889 271 AMst PEL SPE

non ut confundam uos AMstrar $]$ non ut confundam ụọs $75^{*}$, non ut confundamini $75^{\mathrm{C}} 76$, non confundens uos $\mathrm{AMst}^{\mathrm{ed}}$

haec scribo 51545876271 ] scribo haec 61

sed ut $\mathrm{AMst}^{\mathrm{AD}}$ ] sed quasi $\mathrm{AMst}^{\mathrm{ed}}$

filios meos ] filios $5^{1} \mathrm{AMst}^{\mathrm{var}}$

carissimos moneo 5154271

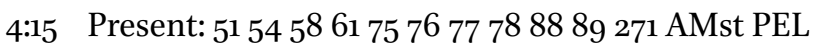

nam si $\mathrm{AMst}^{\mathrm{AD}}$ ] si enim $6175767889 \mathrm{AMst}^{\mathrm{ed}} \mathrm{PEL}^{\mathrm{B}}$, si 77

decem milia ] multa milia $\mathrm{AMst}^{\mathrm{AD}}$

pedagogorum $7576\left(271^{*}\right)$ ] pedagogos 777889

habeatis in christo $271 \mathrm{AMst}^{\mathrm{AD}}$ ] in christo habueritis 61, habueritis in christo

AMsted

sed non multos patres

nam in christo iesu AMst ${ }^{\mathrm{var}}$ ] in uel nam christo enim iesu 77, om. AMst ${ }^{\mathrm{ed}}$ per euangelium 545861 ] + enim AMsted

ego uos genui ] uos genui 58 ; + per fidem quae est in christo iesu domino nostro amen $271^{\dagger}$

4:16 Present: $515458617576777^{1} 88$ 89 AMst PEL

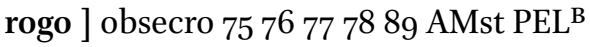

ergo ] om. $61 \mathrm{AMst}^{\mathrm{AD}}$, itaque 75767889 , itaque uel autem 77

uos ] + fratres 58

imitatores mei estote $\left.546175^{*}\right]+$ sicut et ego christi 5461

$\dagger \quad$ 1Cor. 4:15. This is a standard conclusion to a lection in VL 271. 
4:17 Present: 51545861757677788889 AMst PEL

ideo misi ad uos timotheum qui est filius 51545861

meus ] om. AMst ${ }^{\mathrm{var}}$

carissimus 515478 ] rarissimus $\mathrm{PEL}^{\mathrm{var}}$

et fidelis ] + minister 61

in domino qui uos

commonefaciat $\left.54\left(5^{8}\right) 88^{c} \mathrm{AMst}^{\mathrm{var}}\right]$ admonebit $757^{6} 777^{8} 89$, commonefaciet AMst ${ }^{\text {ed }} P E L^{B}$

uias meas quae sunt in 5154586176

christo ] christo iesu 5158 61 AMst PEL ${ }^{\mathrm{B}}$, domino iesu 757778 , domino 76 sicut ubique

in omni ecclesia doceo 5178 ] doceo in omni ecclesia 61 , in omnibus ecclesiis doceo 89 AMst $^{\text {var }}$

4:18 Present: 515458617576777888 89 AMst PEL

tamquam 77 ] + autem $\mathrm{AMst}^{\mathrm{AD}}$

non uenturus sim $(78)\left(88^{*}\right) \mathrm{AMst}^{\mathrm{AD}}$ ] non uenturus essem $61 \mathrm{AMst}^{\mathrm{ted}} \mathrm{PEL}^{\mathrm{B}}$, non essem uenturus AMst ${ }^{\text {var }}$

ad uos sic AMstrar ] ad uos $7778 \mathrm{AMst}^{\mathrm{ed}}$

inflati sunt quidam

4:19 Present: 515458617576777888 89 AMst PEL

ueniam autem cito ] ueniam autem 78 PEL var

ad uos $\mathrm{AMst}^{\mathrm{var}}$ ] om. $89 \mathrm{AMst}^{\text {ed }} \mathrm{PEL}^{\mathrm{B}}$

si dominus ] si deus 75

uoluerit AMst ${ }^{\mathrm{var}}$ ] permiserit AMst ${ }^{\mathrm{ed}}$

et cognoscam non ] et cognoscam $75^{*}$

sermonem ] uerbum 7778

eorum $\langle 76\rangle$ AMst $^{\text {var }}$ ] illorum AMst ${ }^{\text {ed }}$

qui inflati sunt ] inflantium uel qui inflati sunt 77

sed uirtutem

4:2O Present: 5154617576777888 89 AMst PEL tes

non $\mathrm{AMst}^{\mathrm{AD}}$ ] nec uel non 77 , nec $89 \mathrm{AMst}^{\mathrm{ed}} \mathrm{PEL}^{\mathrm{B}}$

enim in ] in TES $S^{\text {ed }}$ om. TES $S^{\mathrm{var}}$

sermone est regnum dei sed

in uirtute ] uirtute $88^{*}$, in uirtutem 89

4:21 Present: 51545861757677788889 AMst PEL

quid $\left.54^{*}\right]+$ enim 58 
uultis in uirga ] uultis in uirgam 75

ueniam ad uos

an $\mathrm{AMst}^{\text {var }}$ ] aut $757689 \mathrm{AMst}^{\mathrm{ed}}$

in caritate

et spiritu AMst ${ }^{\mathrm{ADR}}$ ] spiritus quoque 7576 , spiritus uel spiritu quoque 77 , spirituque 89, spiritus et AMst ${ }^{\text {ed }}$, spiritus AMst ${ }^{\text {var }}$

mansuetudinis $(88)$

5:1 Present: $5^{1} 545^{861} 757677788889$ AMst PEL CAr SPE

omnino $\langle 78\rangle$

auditur inter uos AMst ${ }^{\mathrm{var}}$ ] auditur in uobis $\mathrm{AMst}^{\mathrm{ed}}$, dicitur inter uos CAr, inter uos auditur SPEvar

fornicatio et talis fornicatio 58 ] fornicatio et talis CAr

qualis ] quales $54 * 75$

nec inter gentes ] + nominatur $54^{\mathrm{C}} 58 \mathrm{CAr}$, + uel in gentibus $77,+$ est $\mathrm{AMst}^{\text {ed }}$ ita ut ] ita $75^{*}$, ut $\mathrm{SPE}^{\mathrm{var}}$, abs. CAr

uxorem patris aliquis habeat $\mathrm{AMst}{ }^{\mathrm{AD}}$ ] uxorem patris sui aliquis habeat $5^{8}$

88 , uxorem patris habeat aliquis 7778 , quis uxorem patris habeat $\mathrm{AMst}^{\mathrm{ed}}$, uxorem patris quis habeat $\mathrm{PEL}^{\mathrm{B}}$, uxorem patris sui habeat $P E L^{\mathrm{var}}$, uxorem patris sui habeat aliquis PEL ${ }^{\text {var }}, a b s$. CAr

5:2 Present: $5^{1} 545^{8} 61757677788889$ AMst PEL SPE

et uos inflati estis

et non ] om. $54^{*}$; + uos PEL var

magis ] om. $54^{*}$, potius $7576777889 \mathrm{AMst}^{\mathrm{AD}}$

luctum habuistis ] om. $54^{*}$

ut ] et $\mathrm{PEL}^{\mathrm{B}}$

tollatur $\left(88^{*}\right)$ ] tolleretur 757689 AMst PEL var

de medio uestrum qui

hoc opus $\left(54^{*}\right)$ ] opus hoc 77

fecit ] facit SPEvar

5:3 Present: $5^{1} 545^{8} 617576777888$ 89 AMst PEL sPE

ego quidem ] ego enim 7576 , ego quidem enim 77 , ego equidem 78 ; + paulus 58

absens $\mathrm{AMst}^{\mathrm{ADR}} \mathrm{PEL}^{\text {var }}$ ] quamuis absens 61, ut absens $75767778 \mathrm{PEL}^{\mathrm{AB}}$, absens sum $88^{\mathrm{C}}$, sicut absens $89 \mathrm{AMst}^{\mathrm{ed}}$

corpore praesens autem ${ }_{51} 54^{c} 5^{8} 61767788$ ] corpore $54^{*}$

spiritu ] om. $54^{*}$, spiritus 7576

iam iudicaui $(78)$ ] om. $54^{*} ;+$ eum 61 
ut ] om. $54^{*}$ AMstvar, tamquam 77

praesens 5154586176777888

eum qui ] qui 61, cum qui $P E L^{B}$

sic operatus est $\mathrm{AMst}^{\mathrm{var}}$ ] hoc admisit $\mathrm{AMst}^{\text {ed }}$, ita operatus est $\mathrm{AMst}{ }^{\mathrm{AD}}$

5:4 Present: 51545861757677788889 AMst PEL SPE

in nomine domini nostri ] in nomine domini 61

iesu christi ] iesu 7589

congregatis ] + uel conuenientibus $77,+$ a 89

uobis et

meo spiritu AMst ${ }^{\text {var }}$ ] spiritu meo 757689 AMst $^{\text {ed }}$, cum meo spiritu 77

cum uirtute domini ] + nostri $617576777889 \mathrm{AMst}^{\mathrm{R}} \mathrm{PEL}^{\mathrm{B}}$

iesu ] + christi 61767778 PEL $^{\mathrm{B}}$

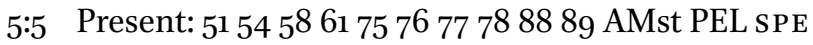

tradere huiusmodi AMst ${ }^{\text {var }}$ ] eum qui talis est tradere 61 PEL $^{\text {var }}$, tradere eum 77 , tradere eiusmodi 78 , tradere eiusmodi hominem 89 , tradere hunc AMst ${ }^{\text {ed }}$, cum quia talis est tradere $P E L^{B}$

satanae $\left.515458767788 \mathrm{SPE}^{\mathrm{var}}\right] \mathrm{om}$. SPE $\mathrm{E}^{\mathrm{ed}}$

in ] om. $\mathrm{SPE}^{\mathrm{var}}$

interitum carnis ut spiritus ] + eius $5^{8}$

saluus sit ] saluus fiat $\mathrm{AMst}{ }^{\mathrm{AD}}$

in die $\left.\mathrm{SPE}^{\mathrm{var}}\right]$ in diem $7576 \mathrm{AMst}^{\mathrm{var}} \mathrm{SPE}^{\mathrm{ed}}$

domini ] + nostri $51545861777^{8} 88^{*} 89$ AMst $^{\mathrm{var}} \mathrm{PEL}^{\mathrm{B}} \mathrm{SPE}^{\mathrm{var}}$

iesu ] + christi $5^{1} 545^{8} 61757^{6} 777^{8}(88) 89$ AMsted $^{\text {ed }} \mathrm{PLL}^{\mathrm{B}} \mathrm{SPE}^{\mathrm{var}}$

5:6 Present: 5154586175767778848889262 AMst PEL SPM SPE non $\mathrm{AMst}^{\mathrm{var}}$ ] fratres non 262, abs. $84 \mathrm{SPM}$, om. $\mathrm{AMst}^{\mathrm{ed}}$

bona ] abs. $84 \mathrm{SPM}$, bona est $\mathrm{AMst}^{\mathrm{R}}$, est bona $\mathrm{SPE}^{\mathrm{var}}$

gloriatio uestra 58 ] abs. 84 SPM, gloriatio $\mathrm{PEL}^{\mathrm{B}}$

nescitis ] fratres nescitis $8488^{\mathrm{C}}$

quia ] quod 78

modicum $\mathrm{SPM}^{\mathrm{var}}$ ] pusillum $\mathrm{SPM}^{\text {ed }}$

fermentum 84 ] fermentatum $S M^{\text {var }}$

totam massam ] abs. 84

corrumpit $6178 \mathrm{SPM}^{\mathrm{var}}$ ] fermentat $777^{\mathrm{gl}} \mathrm{SPM}^{\mathrm{ed}}$, abs. 84

5:7 Present: 51545861757677788889262 AMst PEL tes SPM SPE

expurgate $\left.\left(54^{*}\right)\right]$ + igitur $61262 \mathrm{PEL}^{\text {var }}$

uetus fermentum ut sitis noua 
consparsio ] conspersio $51545^{8} 7678$ AMst $^{\mathrm{var}} \mathrm{PEL}^{\mathrm{B}}$ SPE ${ }^{\mathrm{var}}$; + uel massa 77 sicut estis ] sicut TES ${ }^{\text {var }}$

azymi 515458617888262 ] azyma 89 TES $^{\text {var }}$

etenim $\mathrm{TES}^{\mathrm{var}} \mathrm{SPM}^{\mathrm{var}}$ ] nam et $\mathrm{TES}^{\mathrm{ed}} \mathrm{SPM}^{\mathrm{ed}}$

pascha nostrum immolatus est christus 617889262

5:8 Present: $5^{1} 545^{8} 61757677788889262$ AMst PEL TES SPM SPE

itaque

epulemur ] diem festum celebremus $75\langle 76\rangle 89 \mathrm{AMst}^{\mathrm{AD}}$, diem festum celebremus uel epulemur 77, festa celebremus AMst ${ }^{\text {ed }}$ TE $^{\text {ed }}$, laetemur PEL ${ }^{\text {var, }}$, die festa celebremus TES ${ }^{\mathrm{var}}$, epulemur festa celebremus TES ${ }^{\mathrm{var}}$, sollemnitatem celebremus SPM

non in fermento

ueteri ] uetere $75 \mathrm{PEL}^{\mathrm{A} *}$

neque $\mathrm{AMst}^{\mathrm{AD}}$ ] nec $61 \mathrm{AMst}^{\text {ed }} \mathrm{PEL}^{\mathrm{B}} \mathrm{TES}^{\mathrm{var}}$, non $\mathrm{AMst}^{\mathrm{var}}$

in fermento $\langle 61\rangle$

malitiae 51545876262 ] ueteri nec malitiae $\mathrm{PEL}^{\mathrm{B} *}$

et nequitiae $51545^{8} 76262$ ] om. $88^{*} \mathrm{SPM}^{\mathrm{var}}$; + uel fornicationis 77

sed in azymis sinceritatis et ueritatis 515458617888262

5:9 Present: $51545^{8} 61757677788889262$ AMst PEL sPE

scripsi uobis 89262 ] haec scripsi uobis 58

in epistula 6176777888262 ] in epistulam 75

ne ] non $757689 \mathrm{AMst}^{\mathrm{AD}} \mathrm{PEL}^{\text {var }}$, non uel ne 77 , nec $88^{\mathrm{C}}$

commisceamini fornicariis 54 (61) 262 AMst $^{\text {ed }}$ ] commisceri fornicariis 7576 $89 \mathrm{AMst}^{\mathrm{A}}$, commiscere fornicariis $\mathrm{AMst}^{\mathrm{D}}$

5:10 Present: $51545^{8} 61757677788889262$ AMst PEL sPE

non utique ] + uel omnino 77

fornicariis

huius mundi $88\left(\mathrm{PEL}^{\mathrm{B}}\right)$ ] mundi huius 77 , huiusmodi $88^{\text {alt }} \mathrm{PEL}^{\mathrm{var}}$; + inpudicis 61

aut auaris ] + aut maledicis 58

aut rapacibus aut $\langle 76\rangle]$ et rapacibus aut 757778

idolis seruientibus $5^{1} 5^{8}$ ] idolatris $757678 \mathrm{AMst}^{\mathrm{D}}$, idolatriis uel idolis seruientibus 77 , idolatriis $89 \mathrm{AMst}^{\mathrm{A}}$

alioquin ] alioqui 77

debueratis de hoc mundo ] debueratis de mundo 77 , de hoc mundo debueratis PELvar

exisse $\mathrm{AMst}^{\mathrm{var}}$ ] exire $5461262 \mathrm{AMst}^{\mathrm{ed}} \mathrm{PEL}^{\mathrm{B}}$, exire uel exisse 77 


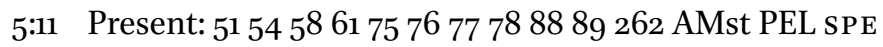

nunc autem scripsi uobis 89262 ] + in epistula $88 *$

non $\mathrm{AMst}^{\mathrm{var}}$ ] ne $78262 \mathrm{AMst}^{\mathrm{ed}}$, ut non $\mathrm{AMst}^{\mathrm{AD}}$

commisceri $547589 \mathrm{AMst}^{\mathrm{var}}$ ] commisceamini 78262 AMst $^{\text {ed }} \mathrm{SPE}^{\mathrm{var}}$, commiscere $P E L^{\text {var }}$ + uos 61 , + uos uel non commisceamini $77,+$ fornicariis 262 , + his $\mathrm{AMst}^{\mathrm{AD}}$

si is qui $54262 \mathrm{AMst}^{\text {var }}$ ] si quis $5^{1} 617576777889 \mathrm{AMst}^{\mathrm{ed}} \mathrm{PEL}^{\mathrm{B}} \mathrm{SPE}^{\mathrm{var}}$, qui $\mathrm{SPE}^{\mathrm{var}}$

\section{frater}

nominatur ] nominetur 757689 , nominatus PELvar $\mathrm{SPE}^{\mathrm{var}}$; + inter uos $88^{*}$ $89 \mathrm{AMst}^{\mathrm{R}}$

est ] et est 61 AMst $^{\text {ed }}$ PEL ${ }^{\text {var }}$, om. 757677788889 AMst $^{\text {var }}$ PEL $^{\text {B }}$; + aut $5^{1} 77$ 78

fornicator aut auarus 54 ] auarus aut fornicator 51 , fornicator 89

aut idolis seruiens 5158 ] aut idolorum cultor 75 76, aut idolatra 78 , om. 89;

+ aut idolorum cultor 77

aut maledicus ] aut maledictus 75

aut ebriosus aut rapax $\left.5461\langle 76\rangle\left(88^{*}\right)\right]$ aut rapax aut ebriosus AMst ${ }^{\text {var }}$

cum eiusmodi AMst ${ }^{\mathrm{AD}}$ ] cum huiusmodi $5458617778 \mathrm{AMst}^{\text {ed }}$

nec ] non uel nec 77, ne $\mathrm{AMst}^{\mathrm{R}}$

cibum sumere $262 \mathrm{AMst}^{\mathrm{var}}$ ] cibum quidem sumere $61 \mathrm{AMst}^{\mathrm{ed}} \mathrm{PEL}^{\mathrm{B}}$, comedere 77

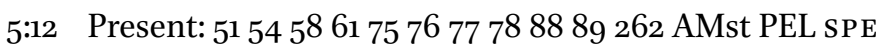

quid $\left.\mathrm{AMst}^{\mathrm{var}}\right]$ si 77 , quo $89 \mathrm{AMst}^{\mathrm{ed}}$

enim mihi 515458262$]+$ est 61

de his 58 ] de iis $\mathrm{AMst}^{\mathrm{R}}$

qui foris sunt iudicare

nonne de his $\left.5^{8}\right]$ non de his 75 , nonne eos $\mathrm{AMst}^{\mathrm{AD}}$

qui intus sunt

uos iudicatis ] iudicatis $5^{8} 75 \mathrm{PEL}^{\mathrm{var}}$, iudicatis uos $61^{*}$

5:13 Present: $51545^{8} 61647576777888$ 89 262 AMst PEL spe

nam eos qui ] qui autem $7576777889 \mathrm{AMst}^{\mathrm{AD}}$, nam ea quae $\mathrm{AMst}^{\mathrm{var}}$, lac. 64

foris sunt deus ] foris sunt dominus $5^{1} 5^{8} \mathrm{AMst}{ }^{\mathrm{var}}$, lac. 64

iudicabit (88) ] iudicauit $5489 \mathrm{AMst}^{\mathrm{var}}$, iudicat 7576 , diiudicabit $\mathrm{AMst}^{\mathrm{R}}$, lac.

64

auferte malum $\left.\left(75^{*}\right)\right]$ lac. 64

ex AMst ${ }^{\text {var }}$ ] a $61 \mathrm{AMst}^{\mathrm{ed}} \mathrm{PEL}^{\mathrm{B}}$, lac. 64, de $757689 \mathrm{AMst}^{\mathrm{AD}}$

uobis ipsis $\langle 64\rangle$ ] uobis 61, uobismet ipsis PEL var 


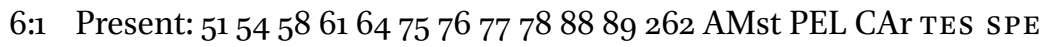
audet $\left.\left(75^{*}\right)\right]$ aude[1] $54^{*}$, lac. 64 , uidet 78 , habet TES ${ }^{\text {var }}$

aliquis $\mathrm{TES}^{\mathrm{var}}$ ] lac. 64, quisquam $\mathrm{AMst}^{\mathrm{AD}} \mathrm{TES}^{\mathrm{ed}}$

uestrum ] lac. 64, nostrum SPEvar

\pm aduersus alterum $\langle 64\rangle 75767889 \mathrm{AMst}^{\text {ed }} \mathrm{PEL}^{\mathrm{A}} \mathrm{TES}^{\mathrm{ed}}$, ad uel aduersus alterum 77 , aduersus alium $\mathrm{AMst}^{\mathrm{A}} \mathrm{TES}^{\mathrm{var}}$

habens negotium $5^{1}$ TES $^{\text {var }}$ ] negotium habens $64\left(75^{*}\right)^{\mathrm{C}} 7677^{\mathrm{C} *}(78) 262$ $\mathrm{AMst}^{\mathrm{AD}} \mathrm{TES}^{\mathrm{ed}}$, habens negotium habens $77^{*}$, habens CAr

aduersus alterum PEL ${ }^{\text {var }}$ ] aduersus aliquem 61, om. 647576777889 AMst $\mathrm{PEL}^{\mathrm{AB}}$ TES, aduersum alterum 88

iudicari TES ${ }^{\mathrm{var}}$ ] iudicare $88^{*} \mathrm{PEL}^{\mathrm{var}}$, iudicium CAr, disceptare $\mathrm{TES}^{\mathrm{ed}}$, discertare TES ${ }^{\mathrm{var}}$, disceptari TES $\mathrm{S}^{\mathrm{var}}$

apud iniquos $51545^{8} 262$ ] ab iniquis 64, apud iniustos $757689 \mathrm{AMst}^{\mathrm{AD}} \mathrm{TES}$, apud uel inter iniquos uel iniustos $77, a b s$. CAr; + iudices $\mathrm{TES}^{\mathrm{var}}$ et non ] abs. CAr

apud sanctos $5154^{*} 586475$ ] apud uel inter sanctos 77 , abs. CAr

6:2 Present: $5^{1} 545^{861} 64757677788889262$ AMst PEL TES SPE an TES ${ }^{\text {var }}$ ] aut 757689 AMst PEL $^{\mathrm{B}}$, om. TES ${ }^{\text {ed }}$

nescitis $\mathrm{AMst}^{\mathrm{AD}}$ ] ignoratis $\mathrm{AMst}^{\mathrm{ed}}$

quoniam TES ${ }^{\text {var }}$ ] quia $5^{1} 64757678 \mathrm{AMst}^{\mathrm{AD}}$ TES $^{\text {ed; }}$ + uel quia 77

sancti

de mundo ] de hoc mundo $515458617576\left(89^{*}\right)^{\mathrm{C}}$ AMst $^{\text {ed }}$ PEL $^{\mathrm{B}}$ TE $^{\mathrm{var}}$ SPE $^{\mathrm{var}}$, mundum 647778 TES $^{\text {ed }}$, de mundo hoc AMst ${ }^{\text {var }}$, hunc mundum AMst $^{\mathrm{R}}$ TES ${ }^{\mathrm{var}}$

\section{iudicabunt}

et ] abs. TES

si $\mathrm{AMst}^{\mathrm{ADR}} \mathrm{PEL}^{\text {var }}$ ] om. $61647576 \mathrm{AMst}^{\mathrm{ed}} \mathrm{PEL}^{\mathrm{AB}}$, abs. TES

in uobis $\mathrm{AMst}^{\mathrm{var}} \mathrm{PEL}^{\mathrm{B}}$ ] in nobis $64 \mathrm{AMst}^{\mathrm{ed}} \mathrm{PEL}^{\mathrm{A} *}$, abs. TES

iudicabitur ] iudicatur $64 \mathrm{AMst}^{\mathrm{R}}$, abs. TES

mundus AMst'var ] hic mundus $61757688^{\mathrm{C}} 89 \mathrm{AMst}^{\mathrm{ed}} \mathrm{PEL}^{\mathrm{B}} \mathrm{SPE}^{\mathrm{var}}$, abs. TES indigni estis $\mathrm{AMst} \mathrm{AD}^{\mathrm{AD}} \mathrm{PEL} \mathrm{L}^{\mathrm{var}}$ ] indigni ergo sunt $61 \mathrm{AMst}^{\mathrm{R}}$, indigni sunt 6489 , indigni sunt ergo AMst ${ }^{\text {ed }} \mathrm{PEL}^{\mathrm{AB}}$, abs. TES

qui de minimis ] huiusmodi qui etiam de minimis $61 \mathrm{AMst}^{\mathrm{ed}}\left(\mathrm{PEL}^{\mathrm{B}}\right)^{\mathrm{C}}$, iudiciorum minimorum $6475\langle 76\rangle 7789$, iudicium iniquorum $\mathrm{AMst}^{\mathrm{AD}}$, abs. TES

iudicetis PEL ${ }^{\text {var }}$ ] iudicent $61 \mathrm{AMst}^{\mathrm{ed}} \mathrm{PEL}^{\mathrm{AB}}$, om. $6475767789 \mathrm{AMst}^{\mathrm{AD}}$, iudicatis PEL var $\mathrm{SPE}^{\mathrm{var}}$, abs. TES 


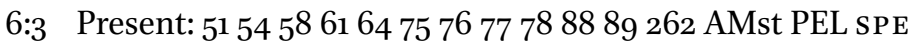

nescitis quoniam ] nescitis quia $64 \mathrm{AMst}^{\mathrm{AD}} \mathrm{PEL}^{\mathrm{B}}$

angelos iudicabimus $\left.61\left(89^{*}\right)\right]$ angelos iudicauimus $\langle 64\rangle 77$, angelos iudicamus 7576 , iudicabimus angelos $\mathrm{PEL}^{\mathrm{B}}$

quanto magis saecularia 51545888262 ] om. 6189 AMst PEL ${ }^{\mathrm{A}}$, nedum saecularia 64 , neque saecularia 7576

6:4 Present: 5154586164757677788889262 AMst PEL SPE saecularia $51545861\langle 64\rangle 7688262$ ] saecularium AMst $^{\mathrm{A}}$ igitur ] om. AMstvar

iudicia si habueritis 262 ] iudicia $88^{*}$

contemptibiles qui $\left.5478(89) 262\left(\mathrm{PEL}^{\mathrm{B}}\right)\right]$ eos qui contemptibiles $64 \mathrm{AMst}^{\mathrm{AD}}$ sunt in ecclesia $516176>8262$

illos ] hos 64, om. 89

\pm ad iudicandum $89 \mathrm{AMst}^{\mathrm{ed}}$, iudicandum $\mathrm{AMst}^{\mathrm{D}}$, iudicandos $\mathrm{AMst}^{\mathrm{A}}$

constituite ] conlocate 64 , constituit PEL ${ }^{\text {var }}$, constitue PEL ${ }^{\text {var }}$, instituite PELvar

ad iudicandum ] om. 647576777889 AMst; + hoc 61

6:5 Present: 5154586164757677788889262 AMst PEL SPE

ad ] ac $\mathrm{PEL}^{\mathrm{B}}$

uerecundiam $61 \mathrm{AMst}^{\mathrm{var}}$ ] reuerentiam $647576 \mathrm{AMst}^{\mathrm{ed}}$, confundendum 89, confusionem $\mathrm{AMst}{ }^{\mathrm{AD}}$

uestram ] uobis $6475767778 \mathrm{AMst}^{\mathrm{AD}}$, uos 89, uerum SPE $\mathrm{var}^{\mathrm{var}}$

dico ] haec dico 89

sic non est inter uos ] si non est inter uos $5^{1}$

sapiens quisquam $\mathrm{AMst}^{\mathrm{AD}}$ ] quisquam sapiens $64777888^{*} 89 \mathrm{AMst}^{\mathrm{ed}}$, sapiens 7576

qui possit iudicare inter 61 ] qui possit inter 64

fratrem suum ] fratrem et fratrem suum 58 , fratres $61 \mathrm{PEL}^{\mathrm{B}}$, proximum uel in medio uel medium fratrem suum 77 , proximum et fratrem eius 78 , fratrem suum et fratrem PEL ${ }^{\text {var; }}$ + iudicare 64

6:6 Present: 5154586164757677788889262 AMst PEL sPE

sed frater cum fratre 262 ] sed fratrem cum fratre 75

iudicio contendit $88^{*}$ ] iudicatur 64 , contenditis uel iudicio contendit 77 , iudicium contendit $S P E^{\mathrm{var}}$, contendit iudicio $\mathrm{SPE}^{\mathrm{var}}$

et hoc apud $\left.5154586475262\left(\mathrm{PEL}^{\mathrm{A}}\right)^{\mathrm{C}}\right]+$ uel inter 77

infideles $\left.75^{*}\right]$ + et non apud fratres 58 , + et non inter sanctos 77 , + et non apud sanctos 78 
6:7 Present: $51545^{8} 61647576\langle 77\rangle^{\dagger} 788889262$ AMst PEL TES SPE iam ] om. $75^{*}$

quidem ] + in uobis AMst ${ }^{\text {var }}$

omnino delictum 61 ] delictum in totum TES ${ }^{\text {ed }}$, delictum tantum TES $S^{\text {var }}$

est in uobis TES ${ }^{\text {var }}$ ] est $64 \mathrm{AMst}^{\mathrm{var}}$, est uobis $757689 \mathrm{AMst}^{\mathrm{AD}}$, in uobis est AMst $^{\text {ed }}$ TES $S^{\text {ed }}$

quod TES $\left.{ }^{\text {var }}\right]$ q[1-3] $54^{*}$, quia 5864757689 AMst $^{\mathrm{AD}} \mathrm{TES}^{\text {ed }}$

iudicia habetis $\langle 64\rangle$ ] iudicị̂s habetis $88^{*}$, iudicium habetis TE ${ }^{\mathrm{var}}$

inter uos ] uobiscum 64, cum inuicem $\mathrm{TES}^{\text {ed }}$, inuicem $\mathrm{TES}^{\mathrm{var}}$, aduersus inter uos TES ${ }^{\text {var }}$, in inuicem TES var

quare non magis ] cur non magis $P E L^{\text {var }}$

iniuriam ] iniquitatem 64

accipitis AMst ${ }^{\mathrm{var}} \mathrm{TES}^{\mathrm{var}}$ ] accipistis 61, patimini $64757689 \mathrm{AMst}^{\text {ed }}$ TES $^{\text {ed, }}$, accepistis $P E L^{B}$, patiemini TES ${ }^{\text {var }}$, sustinetis TES ${ }^{\text {var }}$

quare ] cur PEL ${ }^{\text {var }}$, aut quare TES ${ }^{\text {var }}$, et quare TES ${ }^{\text {var }}$

non magis ] non poti $[u] \mathrm{s} 64$

fraudem patimini $\left(88^{\mathrm{C}}\right) \mathrm{AMst}^{\mathrm{var}}$ ] in fraude patimini 54 , fraudem patiemini $61\left(88^{*}\right)$ PEL $^{\text {var }}$ TES $^{\text {var }}$, fraudamini 64757689 AMst $^{\text {ed }}$, et fraudamini TE $S^{\text {var }}$

6:8 Present: $5^{1} 545^{861647576\langle 77\rangle} 788889262$ AMst PEL TES SPE

sed 262 ] om. TES ${ }^{\text {var }} ;+$ et 58 TES ${ }^{\text {var }}$

uos ] om. 61; + magis 54, + fraudatis et 61757689 AMst $^{\text {ed }}$ PEL $^{B}$ TES ${ }^{\text {var }}$

iniuriam facitis ] iniquitatem facitis 64

et fraudatis $\mathrm{AMst}^{\mathrm{var}}$ ] om. $61757689 \mathrm{AMst}^{\mathrm{ed}} \mathrm{PEL}^{\mathrm{B}}$, et fraudem TES $\mathrm{S}^{\mathrm{var}}$

et hoc ] om. TES var $^{\text {tor }}$

fratribus TES $S^{\mathrm{var}}$ ] fratres $64 \mathrm{TES}^{\mathrm{ed}}$, circa fratres TES $\mathrm{S}^{\mathrm{var}}$

6:9 Present: $51545861647576\langle 77\rangle 788889262$ AMst PEL TES SPM SPE

an nescitis TES ${ }^{\mathrm{var}}$ ] aut nescitis $757689 \mathrm{AMst}^{\mathrm{AD}} \mathrm{TES}^{\mathrm{ed}}$, abs. SPM

quia PEL var TES $^{\text {var }}$ ] quoniam 61757689 PEL $^{\mathrm{AB}}$ TES $^{\text {ed }}$, abs. SPM

iniqui TES ${ }^{\text {var }}$ ] iniusti 64 TES $^{\text {ed }}$, abs. SPM

regnum dei non ] abs. SPM

possidebunt $61\left(88^{*}\right)$ TES $^{\text {var }}$ ] hereditabunt 64 , consequentur TES ${ }^{\text {ed }}$, consequuntur TES ${ }^{\text {var }}$, abs. SPM

nolite errare ] nolite ergo errare PEL ${ }^{\mathrm{var}}$, abs. TES

neque ] nam neque AMst ${ }^{\text {var }}$, numquid $P E L^{B}$

fornicarii 61262 ] fornicatores 64 TES $^{\mathrm{var}}$, inpudici 757689

$\dagger \quad$ 1Cor. 6:7-14. There is a blank space in VL 77 where these verses should be, implying that they were present in the Latin exemplar. 
neque idolis seruientes $515861\left(75^{*}\right) \mathrm{SPM}^{\mathrm{var}}$ ] neque idoli seruientes $64 \mathrm{PEL}^{\mathrm{A}}$, neque idola colentes $\mathrm{SP}^{\mathrm{ed}}$

neque adulteri neque molles $5^{8}$ ] neque molles neque adulteri S PM neque masculorum

concubitores $\langle 76\rangle$ TES $^{\mathrm{var}}$ ] cubitores $88^{*}$ TES $^{\mathrm{var}}$, concupitores PEL ${ }^{\mathrm{var}}$, adpetitores TES ${ }^{\text {ed }}$

6:10 Present: $51545861647576\langle 77\rangle 788889262$ AMst PEL TES SPM SPE neque fures neque $\mathrm{SPM}^{\mathrm{ed} 2}$ ] non fures non $\mathrm{SPM}^{\mathrm{ed}}$

auari 262 TES $^{\text {var }}$ ] fraudulenti TES $S^{\text {ed }}$, fraudulentes TES ${ }^{\text {var }}$, fraudatores TES ${ }^{\text {var }}$ neque ebriosi $61 \mathrm{SPM}^{\mathrm{var}}$ ] non ebriaci $\mathrm{SPM}^{\mathrm{ed}}$

neque $S \mathrm{PM}^{\mathrm{var}}$ ] om. PEL ${ }^{\mathrm{var}}$, non $\mathrm{SPM}^{\mathrm{ed}}$

maledici 61 ] maledicti $75 \mathrm{SPM}^{\mathrm{var}}$, om. PEL var

neque rapaces $\mathrm{TES}^{\mathrm{var}} \mathrm{SPM}^{\mathrm{var}}$ ] neque raptores $\mathrm{TE} \mathrm{S}^{\mathrm{ed}}$, non rapaces $S P \mathrm{M}^{\mathrm{ed}}, \mathrm{om}$. SPM ${ }^{\text {ed2 }}$

regnum dei

possidebunt 262 TES $^{\text {var }}$ ] non possidebunt 58 , consequentur TES ${ }^{\text {ed }}$, consequuntur TES var

6:11 Present: $51545^{8} 61647576\langle 77\rangle 788889262$ AMst PEL TES SPM

et ] sed (75) 7689

haec 515476262 ] om. 58 , hoc $\mathrm{AMst}^{\mathrm{R}}$

quidam PEL ${ }^{\mathrm{A}}$ ] quidem $5^{1} 617888262 \mathrm{AMst}^{\mathrm{var}} \mathrm{PEL}^{\mathrm{B}}$ TES ${ }^{\mathrm{var}}$, aliquando quidem 54 , quidem haec 58 , om. 757689 TES $^{\text {var }}$

fuistis ] fuistis aliquando 61, aliquando fuistis $757689 \mathrm{TES}^{\mathrm{var}}$

sed abluti estis 262] om. TES $\mathrm{S}^{\mathrm{var}}$

sed 262 ] om. $88^{\mathrm{C}} \mathrm{SPM}^{\mathrm{var}}$, et TES $\mathrm{S}^{\mathrm{var}}$

sanctificati estis $(78)] \mathrm{om}$. SPM $\mathrm{Sar}^{\mathrm{va}}$

sed iustificati estis $\left.(78) 262 \mathrm{SPM}^{\mathrm{var}}\right] \mathrm{om}$. $\mathrm{PEL}^{\mathrm{A} * \mathrm{~B}} \mathrm{TES}^{\mathrm{var}} \mathrm{SPM}^{\mathrm{ed}}$

in nomine domini

nostri AMst ${ }^{\text {var }}$ TES $^{\text {var }}$ ] dei nostri 51 SPM $^{\text {var }}$, om. 7576 AMst $^{\text {ed }}$ PEL $^{\text {var }}$ TES $^{\text {ed }}$

iesu christi ] om. SPM $^{\text {var; }}$ + deo et patri 262

et in $\mathrm{AMst}^{\mathrm{AD}}$ ] et $7889 \mathrm{AMst}^{\mathrm{ed}} \mathrm{PEL}^{\text {var }} \mathrm{TES}^{\mathrm{var}}$, abs. 262

spiritu ] abs. 262; + sancto TES ${ }^{\mathrm{var}}$

dei nostri ] abs. 262, dei mei PEL ${ }^{\mathrm{A} *}$, dei noster PEL ${ }^{\mathrm{AC}}$

6:12 Present: $5^{1} 545^{8} 61647576\langle 77\rangle 7^{8} 88$ 89 AMst PEL

omnia mihi licent $51545^{8}$ ] omnia mihi licita sunt 64 , om. AMstrar

sed non omnia ] om. AMst ${ }^{\mathrm{var}}$, et non omnia PEL ${ }^{\mathrm{var}}$

expediunt ] utilia sunt $61 \mathrm{PEL}^{\mathrm{var}}$, om. AMst ${ }^{\mathrm{var}}$, utilia $\mathrm{PEL}^{\mathrm{B}}$ 
omnia mihi licent $51545^{8}$ ] omnia mihi licita sunt 64

sed ego ] sed 78

sub nullius ] nullius 7576 , non $89 \mathrm{AMst}^{\mathrm{AD}}$

redigar potestate $61 \mathrm{AMst}^{\mathrm{var}}$ ] redigar a quibus $(75) 76$, redigar potestatem 88 $\mathrm{AMst}^{\text {ed }} \mathrm{PEL}^{\mathrm{A} *}$, redigar sub potestate ullius 89 , redigar sub potestate illius $\mathrm{AMst}^{\mathrm{AD}}$, potestate redigor PEL ${ }^{\text {var }}$

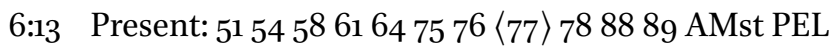

esca uentri 61 ] escae 7576

et uenter escis 6176 ] lac. 64

deus autem et $\langle 64\rangle$ ] deus autem $61 \mathrm{AMst}^{\mathrm{var}}$

hunc et ] lac. 64 , hanc et $757^{\mathrm{C}}$, haec et $89 \mathrm{PEL}^{\mathrm{B}}$, hoc et PEL var

haec ] hanc $5^{1} 54^{\mathrm{C}} 5^{8} 6188 \mathrm{AMst}^{\mathrm{var}} \mathrm{PEL}{ }^{\mathrm{var}}$, om. $54^{*}$, lac. 64, hunc $89 \mathrm{PEL}^{\mathrm{B}}$, illam AMst ${ }^{\mathrm{AD}}$, has PEL var

destruet corpus autem non 61 ] lac. 64

fornicationi 58 ] lac. 64 , fornicatione $88^{*}$, fornicatio $\mathrm{PEL}^{\mathrm{B}}$

sed domino ] sed dominus 61, sed deo AMstrar

et dominus corpori

6:14 Present: $51545861647576\langle 77\rangle 788889$ AMst PEL

deus uero ] deus autem $647576 \mathrm{AMst}^{\mathrm{AD}}$

et ] qui $61 \mathrm{PEL}^{\mathrm{B}}$, om. $89 \mathrm{PEL}^{\mathrm{var}}$

dominum ] om. 61 $\mathrm{PEL}^{\mathrm{B}}$, dominum iesum $\mathrm{AMst}^{\mathrm{AD}}$, deum PEL var

suscitauit $\langle 76\rangle]$ suscitabit 64

\pm dominum nostrum iesum christum 61 , dominum nostrum PEL $^{B}$

et nos ] nos PELvar; + quoque 61 PEL $^{\mathrm{AB}}$, + cum illo $\mathrm{AMst}^{\mathrm{AD}}$

suscitabit ] suscitauit $54^{*} 6489 \mathrm{AMst}^{\mathrm{var}} \mathrm{PEL}^{\text {var }}$, suscitat $757^{6}$

per uirtutem suam ] per potentiam suam 64

6:15 Present: 5154586164757677788889 AMst PEL TES SPM SPE

nescitis ] an nescitis $617778 \mathrm{PEL}^{\mathrm{B}}$

quoniam AMst ${ }^{\text {var }}$ TES $^{\text {var }}$ SPM $^{\text {var }}$ ] quia 647576777889 AMsted $^{\text {ed }}$ TES $^{\text {ed }}$ $\mathrm{SPM}^{\mathrm{ed}}$

corpora uestra membra 5875

christi sunt ] sunt christi 51586478 AMstvar $^{\mathrm{vaEL}}{ }^{\mathrm{var}} \mathrm{TES}^{\mathrm{var}} \mathrm{SPM}^{\mathrm{var}}$; + faciam membra meretricis absit 77

tollens 78 ] tolles $5^{8} \mathrm{SPM}^{\mathrm{var}}$, an tollens $777^{\mathrm{gl}}$, tollam $\mathrm{AMst}^{\mathrm{R}} \mathrm{TES}^{\mathrm{var}}$, auferens TES ${ }^{\text {ed }}$, aufers TES var $^{\text {var }}$

ergo membra christi $\left.75 \mathrm{TES}^{\mathrm{var}}\right]$ membra christi TES $\mathrm{S}^{\mathrm{ed}}$

faciam membra $\left(88^{*}\right)$ ] faciens membra $89 \mathrm{TES}^{\mathrm{var}}$, et faciam membra $\mathrm{AMst}^{\mathrm{R}}$, facies membra TEs ${ }^{\mathrm{var}}$ 
meretricis $6188 \mathrm{TES}^{\mathrm{var}}$ ] pọ̣ṇịçẹtricis $77,^{\dagger}$ fornicariae $\mathrm{TES} \mathrm{S}^{\mathrm{ed}}$, fornicatoriae $\mathrm{TES}^{\mathrm{var}}$, fornicationis TES $\mathrm{var}^{\mathrm{v}}$

absit TES ${ }^{\text {var }}$ ] om. TES ${ }^{\text {ed }}$

6:16 Present: $5^{1} 545^{8} 6164757677788889$ AMst PEL TES SPM SPE an ] om. $64 \mathrm{PEL}^{\mathrm{B}}$ SPM, aut $75767889 \mathrm{AMst}^{\mathrm{AD}}$ TES, an uel aut 77 nescitis quoniam qui ] nescitis quia qui 6475767889 AMst TES SPM adheret (54) 88 AMst $^{\text {ed }}$ ] se iungit 757689 , adheret uel iungit 77 , coniungit se $A M s t^{\mathrm{AD}} \mathrm{TES}^{\mathrm{var}}$, inheret $\mathrm{PEL}^{\mathrm{B}}$, conglutinantur TES $S^{\mathrm{ed}}$, glutinantur TE ${ }^{\mathrm{var}}$, iunguntur TES ${ }^{\mathrm{var}}$, se coniungunt TE ${ }^{\mathrm{var}}$, se iungunt TES ${ }^{\mathrm{var}}$, iungit Se SPM meretrici $6177 \mathrm{TES}^{\mathrm{var}}$ ] fornicariae TES ${ }^{\mathrm{ed}}$, fornicari TES $\mathrm{var}^{\mathrm{var}}$ unum corpus ] corporis unitate $\mathrm{PEL}^{\mathrm{B}}$ efficitur AMst ${ }^{\text {var }}$ SPM $^{\text {var }}$ ] est 647576777889 AMst $^{\mathrm{AD}}$ TES $^{\mathrm{var}}$ SPM $^{\mathrm{ed}}$, fit AMst $^{\text {ed }}$, connectitur PEL ${ }^{\mathrm{B}}$, sunt TES ${ }^{\text {ed }}$, facit TES ${ }^{\text {var }}$ \pm sicut in genesi dicit $\mathrm{PEL}^{\mathrm{B}}$ erunt ] erant PEL var enim $\left.S P E^{\mathrm{var}}\right]$ om. AMst $S P E^{\mathrm{ed}}$, autem TES ${ }^{\mathrm{var}}$ inquit $6475\left(88^{*}\right) 89$ TES $^{\text {var }}$ ] om. TES ${ }^{\text {ed }}$ SPM duo in carne una ] ambo in carnem unam TES $S^{\mathrm{var}}$

6:17 Present: $51545^{8} 61647576777888$ 89 AMst PEL TES SPM SPE qui autem ] qui enim TES ${ }^{\text {var }}$ adheret $A M s t^{e d} S P E$ ] se iungit 757689 , adheret uel iungit 77 , adiungit se $\mathrm{AMst}^{\mathrm{AD}} \mathrm{SPM}^{\mathrm{var}}$, se coniunxerint $\mathrm{TE} \mathrm{S}^{\mathrm{ed}}$, se coniungit $\mathrm{TE} \mathrm{S}^{\mathrm{var}}$, se coniunxerit TES ${ }^{\text {var }}$, iungit se $S P M^{\text {ed }}$

domino ] deo AMst ${ }^{\text {var }} \mathrm{TES}^{\mathrm{var}} \mathrm{SPM}^{\mathrm{var}}$, dominum TES ${ }^{\mathrm{var}}$ unus spiritus ] unum efficit spiritum TES ${ }^{\mathrm{var}}$, uno spiritu TES ${ }^{\mathrm{var}}$ est ] sunt TES $S^{\text {ed }}$, aliter unum corpus sunt TE $S^{\text {var }}$

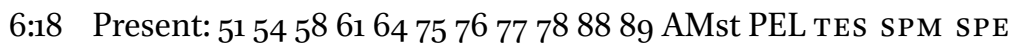
fugite fornicationem 58 ] fugite fornicatione $\mathrm{PEL}^{\mathrm{A}}$, abs. TES omne $\left.\left(64^{*}\right)\right]+$ enim $5^{1} 5^{8}$ peccatum ] delictum TES quodcumque 7788$]$ qu[em]cumque $64^{*}$, quod 89 TE $S^{\text {var }}$ fecerit ] peccauit $S \mathrm{PE}^{\mathrm{var}}$ homo extra corpus ] + suum $\mathrm{AMst}^{\mathrm{AD}}$ est ] peccat $\mathrm{AMst}^{\mathrm{AD}}$

$\dagger \quad$ 1Cor. 6:15. The copyist of VL 77 has written part of the Greek word below as the Latin gloss. 


\section{qui autem fornicatur in}

corpus suum ] corpus proprium 64, suum corpus 77 , carne sua TE S $^{\text {var }}$ peccat

6:19 Present: $5^{1} 545^{8} 6164757677788889$ AMst PEL TES $^{\dagger}$ FOR SPM SPE ${ }^{\dagger}$ an $\mathrm{SPM}^{\mathrm{var}}$ ] aut $757689 \mathrm{AMst}^{\mathrm{A}} \mathrm{SPM}^{\mathrm{ed}}$, abs. TES FOR

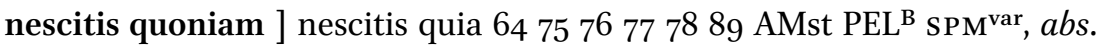
TES FOR

membra uestra ] corpus christi 61, corpus uestrum $647576777889 \mathrm{AMst}^{\mathrm{A}}$, corpora uestra AMst ${ }^{\text {ed }}$ PEL $^{\text {var }}$, corpus $\mathrm{PEL}^{\mathrm{B}}$, abs. TES FOR

templum ] abs. TES FOR; + dei 61 PEL $^{\mathrm{B}}$

est ] sunt $54^{\mathrm{C}} \mathrm{SPM}{ }^{\mathrm{var}} \mathrm{SPE}^{\mathrm{var}}$, om. 64757677 , abs. TES FOR

spiritus sancti $\left(54^{*}\right)$ ] om. $616475767778 \mathrm{AMst}^{\mathrm{A}} \mathrm{PEL}^{\mathrm{B}}$, eius 89 , spiritus eius SPE ${ }^{\mathrm{var}}, a b s$. TES FOR

qui in uobis est $\mathrm{AMst}^{\mathrm{AR}}$ ] quod in uobis est 61, in uobis 64, quod in uobis 75 76 PEL $^{\mathrm{B}}$, om. $88^{*}$ AMst ${ }^{\mathrm{ed}}$, abs. TES FOR

\pm spiritus sanctus est 61, spiritus sancti est 64, sancti spiritus est 757677 $\mathrm{PEL}^{\mathrm{B}}$, spiritus sancti $7889 \mathrm{AMst}^{\mathrm{A}}$

quem ] quod uel quem 77, quod $\mathrm{AMst}^{\mathrm{A}} \mathrm{PEL}^{\mathrm{B}}$, abs. TES FOR

habetis (78) ] est $\mathrm{AMst}^{\mathrm{A}}$, habet PEL ${ }^{\mathrm{var}}$, abs. TES FOR

a deo et ] deus et $\mathrm{AMst}^{\mathrm{A}}$, abs. TES FOR

non ] + enim FOR ${ }^{\text {var }}$

estis uestri ] sunt uestri $5^{8^{\mathrm{C}}}$

6:2O Present: $5^{1} 545^{86164757677788889 \text { AMst PEL TES }^{\dagger} \text { FOR SPM SPE }}{ }^{\dagger}$ empti enim estis $54\left(89^{*}\right)$ ] empti estis enim 77

pretio magno $\left.5154\left(64^{\mathrm{C}}\right) 89 \mathrm{AMst}^{\mathrm{var}} \mathrm{TES}^{\mathrm{var}} \mathrm{FOR}^{\mathrm{var}} \mathrm{SPM}^{\mathrm{var}}\right]$ magno $64^{*} \mathrm{TES}^{\mathrm{ed}}$ FOR $^{\text {ed }}$, pretio $75767778 \mathrm{AMst}^{\text {ed }}$ TES $^{\text {var }}$ SPM $^{\text {ed }}$, magno pretio TES ${ }^{\text {var }}$ FOR $^{\text {var }}$ $\mathrm{SPM}^{\mathrm{ed}}$, magno sanguinis christi pretio TES ${ }^{\mathrm{var}}$

glorificate $\mathrm{AMst}^{\mathrm{AD}} \mathrm{TES}^{\mathrm{var}} \mathrm{FOR}^{\mathrm{var}}$ ] clarificate $\mathrm{AMst}^{\mathrm{ed}} \mathrm{TES}^{\mathrm{ed}} \mathrm{FOR}^{\mathrm{ed}}$

et ] ergo 64, om. 7576

portate $\mathrm{SPM}^{\mathrm{var}}$ ] om. 647576 , tollite $\mathrm{SPM}^{\mathrm{ed}}$

deum SPM ${ }^{\text {ed2 }} \mathrm{SPE}^{\mathrm{var}}$ ] dominum $6189 \mathrm{AMst}^{\mathrm{var}} \mathrm{PEL}^{\mathrm{var}} \mathrm{TES}^{\mathrm{var}} \mathrm{FOR}^{\mathrm{var}} \mathrm{SPM}^{\mathrm{ed}}$ $S P E^{\text {ed }}$

in corpore TES $S^{\text {ed }}$ om. $88^{*}$, in corde $\mathrm{TES}^{\mathrm{var}}$

uestro PEL var $]$ om. 88* AMst $^{\text {var }}$ PEL $^{\mathrm{B}}$

$\dagger \quad 1$ Cor. 6:19-20. These verses are attested twice in TES, once as an addition in certain manuscripts (3.63.2) and once in the body of the text (3.11.30). They also occur twice in the editorial text of SPM. 
7:1 Present: 5154586164757677788889 AMst PEL CAr TEs SPE

de quibus autem $\mathrm{AMst}^{\mathrm{var}}$ ] de his autem quae $64 \mathrm{AMst}^{\mathrm{ed}}$, de iis $\mathrm{AMst}^{\mathrm{R}}, a b s$. TES

scripsistis $6478\langle\mathrm{CAr}\rangle]$ abs. TES; + mihi $51545^{8} 617576777^{8} 88^{\mathrm{C}} 89$ AMst PEL $^{\mathrm{AB}}$ CAr SPE

bonum est homini ] bonum est hominem TES ${ }^{\text {var }}$

mulierem non tangere $\left.61\left(64^{*}\right) \mathrm{AMst}^{\mathrm{var}} \mathrm{TES}^{\mathrm{var}}\right]$ mulierem non contingere $\mathrm{AMst}^{\mathrm{ed}} \mathrm{TES}^{\mathrm{ed}}$, non tangere mulierem $\mathrm{SPE}^{\mathrm{var}}$

7:2 Present: 51545861647576777888 89 AMst PEL TES SPE propter $\mathrm{AMst}^{\mathrm{AD}}$ ] propter autem 77 , tamen propter AMsted fornicationes 58 ] fornicationem 51546177788889 AMst PEL $^{\mathrm{A}} \mathrm{TES} \mathrm{S}^{\mathrm{var}} \mathrm{SPE}^{\mathrm{var}}$, incontinentiam PEL $^{\mathrm{B}} \mathrm{TES}^{\mathrm{var}}$

autem unusquisque $\mathrm{AMst} \mathrm{AD}^{\mathrm{AD}}$ ] unusquisque $77 \mathrm{AMst}^{\mathrm{ed}}$

suam uxorem habeat $\mathrm{TES} \mathrm{S}^{\mathrm{var}}$ ] uxorem suam habeat $5864 \mathrm{AMst}^{\mathrm{R}} \mathrm{TES}^{\mathrm{ed}}$, suam uxorem accipiat PEL ${ }^{\text {var }}$, uxorem habeat suam TE $S^{\text {var }}$

et unaquaeque suum uirum habeat $\left.5154^{c} 5^{8}(61) 75^{*} 76 \mathrm{PEL}^{\mathrm{var}} \mathrm{TES} \mathrm{S}^{\mathrm{var}}\right]$ et unaquaeque uirum suum habeat $64 \mathrm{TES}^{\mathrm{ed}}$, om. $7778 \mathrm{AMst}^{\mathrm{var}} \mathrm{PEL}^{\mathrm{A}}$

7:3 Present: 51545861647576777888 89 AMst PEL TES SPE uxori uir debitum reddat $\left.\left(88^{*}\right)\left(\mathrm{PEL}^{\mathrm{A}}\right)\right]$ et uxori uir debitum reddat $\mathrm{TE} \mathrm{S}^{\mathrm{var}}$ similiter autem $\mathrm{AMst}^{\text {var }}$ ] similiter aut $88 \mathrm{PEL}^{\mathrm{B}}$, similiter $\mathrm{AMst}^{\text {ed }}$ TES et uxor uiro $\left(77^{*}\right)$

7:4 Present: 51545861647576777888 89 AMst PEL TES SPE mulier TES $S^{\text {var }}$ ] uxor 89 TES $^{\text {ed; }}+$ autem AMst ${ }^{\text {var }}$ sui corporis TES ${ }^{\text {var }}$ ] corporis sui TE $S^{\text {ed }}$ potestatem non habet ] potestatem non habeat $5464 \mathrm{AMst}^{\mathrm{R}} \mathrm{TES}^{\mathrm{var}}$, non habet potestatem $7778 ;+$ uualtit $^{\dagger} 78$ sed uir ] et uir PEL var similiter autem et $\mathrm{AMst}^{\mathrm{AD}}$ ] similiter et $89 \mathrm{AMst}^{\text {ed }} \mathrm{PEL}^{\mathrm{B}}$ TES uir sui corporis $\mathrm{TES}^{\mathrm{var}}$ ] uir proprii corporis 78 , uir corporis sui TES ${ }^{\text {ed }}$ potestatem non habet ] potestatem non habeat $5464 \mathrm{AMst}^{\mathrm{R}} \mathrm{TES}^{\mathrm{var}}$, non habet potestatem 7778 sed mulier 54 TES $\left.^{\text {var }}\right]$ om. PEL var, sed uxor TES ${ }^{\text {ed }}$

$\dagger \quad{ }_{1}$ Cor. 7:4. The meaning of this word is unclear. 
7:5 Present: $51545^{8} 61647576777888$ 89 AMst PEL TES SPE

nolite ] noli TES ${ }^{\text {var }}$

fraudare inuicem ] fraudari inuicem 54, inuicem fraudare 64, fraudare in inuicem PEL var

nisi forte $\left.\mathrm{AMst}^{\mathrm{var}} \mathrm{TES}^{\mathrm{var}}\right]$ nisi $64 \mathrm{AMst}^{\mathrm{ed}} \mathrm{TES}^{\mathrm{ed}}$

ex consensu (64) TES ${ }^{\text {var }}$ ] ex conuentione et TES ${ }^{\text {ed }}$

ad tempus ut ] ut ad tempus TES ${ }^{\text {var }}$

uacetis orationi et iterum 58 ] uacetis oratione et iterum $\mathrm{AMst}^{\mathrm{A}}$

reuertimini $\mathrm{AMst}^{\mathrm{var}}$ TES $^{\mathrm{var}}$ ] om. $61647576777889 \mathrm{AMst}^{\mathrm{ed}} \mathrm{PEL}^{\mathrm{B}}$ TES $^{\mathrm{ed}}$, redite $\mathrm{TES}^{\mathrm{var}}$, conuertemini TES ${ }^{\mathrm{var}}$, reuertamini $S P \mathrm{E}^{\mathrm{var}}$

in id ipsum $\mathrm{AMst}^{\mathrm{AD}}$ ] ad id ipsum $64757689 \mathrm{AMst}^{\text {ed }}$ TES $^{\text {ed }}$, ad ipsum $\mathrm{TES}^{\mathrm{var}}$ \pm conuertemini 61 , estote 64 , reuertimini uel sitis 77 , reuertimini $78 \mathrm{AMst}^{\text {ed }}$ $\mathrm{PEL}^{\mathrm{B}}$, sitis $89 \mathrm{AMst}^{\mathrm{AD}}$

ne ] ut ne 7778

temptet uos ] uos temptet 647589 AMst TES $^{\text {var }}$, temptet uel uret uos 77 satanas propter $5^{1} 5^{8}$

incontinentiam $\mathrm{AMst}^{\mathrm{AD}}$ ] intemperantiam $647576 \mathrm{AMst}^{\text {ed }} \mathrm{PEL}^{\mathrm{B}}$

uestram TES ${ }^{\text {var }}$ ] om. TES ${ }^{\text {ed }}$ + et inanis sit labor uester TES $S^{\text {var }}$

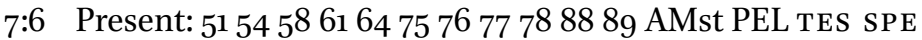

hoc autem TES $\left.{ }^{\text {var }}\right]$ hoc TES $S^{\text {ed }}$

$\operatorname{dico}\langle 64\rangle]$ om. AMst ${ }^{\mathrm{var}} ;+$ uobis TES $\mathrm{S}^{\mathrm{var}}$

secundum

indulgentiam 58 TES $^{\text {var }}$ ] ueniam 64 TES $^{\text {ed }}$, consilium 757678 TES $^{\text {var }}$, conscientiam uel consilium 77 , indulgentiam consilium 89

non secundum $\langle 64\rangle]$ et non secundum PEL var

imperium TES $^{\text {var }}$ ] iussum TES ${ }^{\text {ed }}$

7:7 Present: $51545^{8} 61647576777888$ 89 AMst PEL TES SPE

uolo autem AMst ${ }^{\text {var }}$ ] uolebam autem $\mathrm{AMst}^{\mathrm{ed}}$, uolo $\mathrm{TES}^{\mathrm{var}}$, nam uolo TES $\mathrm{S}^{\mathrm{var}}$ omnes homines

esse sicut ] esse sicut et $757677 \mathrm{TES}^{\mathrm{ed}}$, esse secundum AMst ${ }^{\mathrm{var}}$, sic esse ut TES var $^{2}$

me ipsum TES $\left.{ }^{\text {var }}\right]$ me TES ${ }^{\text {ed }}$

sed unusquisque proprium $\langle 64\rangle\left(88^{*}\right)$

habet donum AMst ${ }^{\text {var }}$ ] donum habet $5^{8} 6489$ AMst $^{\text {ed }}$ PEL ${ }^{\text {var }}$, habet charisma TES $S^{\text {ed }}$, habeat donum TES ${ }^{\text {var }}$

ex $\mathrm{AMst}^{\mathrm{R}}$ ] a $6475767789 \mathrm{AMst}^{\mathrm{ed}}$ TES

deo ] domino $75^{*}$

alius quidem ] lac. 64, alius $757689 \mathrm{AMst}^{\mathrm{AD}} \mathrm{TES}^{\mathrm{ed}}$, unus autem TES ${ }^{\mathrm{var}}$ 
sic ] lac. 64, sicut TES ${ }^{\text {var }}$

alius uero ] alius autem $5^{8} 7576 \mathrm{AMst}^{\mathrm{AD}} \mathrm{TES}^{\mathrm{ed}}$, alius quidem 64, et alius 89 , alius non TES ${ }^{\text {var }}$

sic ] sicut TES var

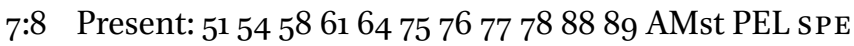

dico autem

non nuptis $\mathrm{AMst}^{\mathrm{var}}$ ] innuptis $61\langle 64\rangle \mathrm{AMst}^{\mathrm{ed}}$, his qui sine uxoribus sunt 75 $7689 \mathrm{AMst}^{\mathrm{AD}}$, his qui sine uxoribus sunt uel non nuptis 77

et uiduis bonum

est illis si ] esse illis si 64, illis si AMst ${ }^{\text {var }} \mathrm{SPE}^{\mathrm{var}}$

sic maneant ] sic permaneant $5^{1} 61 \mathrm{AMst}^{\mathrm{var}} \mathrm{PEL}^{\mathrm{B}} \mathrm{SPE}^{\mathrm{var}}$, sic permanserint $5^{8}$

$\langle 64\rangle 78 \mathrm{AMst}^{\mathrm{ed}}$, sic manserint 757689 , permanserint sic 77 , ita permanserint $\mathrm{AMst}^{\mathrm{AD}}$, sic permanent $\mathrm{PEL}^{\mathrm{var}}$, sic maneat $\mathrm{SPE}^{\mathrm{var}}$

sicut et ego

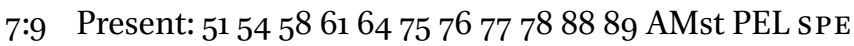

quod si ] si uero 77 , si autem $\mathrm{AMst}^{\mathrm{AD}}$

non se continent $\left.A M s t^{A D} S E^{\text {var }}\right]$ se non continent $58\langle 64\rangle 78 \mathrm{AMst}^{\text {ed }} \mathrm{PEL}^{\mathrm{var}}$ $S_{P E}{ }^{\text {ed }}$, non continent se 6177 , se non continet AMst ${ }^{\text {var }}$, non continent PEL $^{B}$

nubant ] nubat $\mathrm{AMst}^{\mathrm{var}}$, inibant $\mathrm{PEL}^{\mathrm{B}}$, nubent $\mathrm{SPE}^{\mathrm{var}}$

melius est enim AMst ${ }^{\text {var }}$ ] melius est $6178 \mathrm{AMst}^{\text {ed }}$, melius enim est 76 , melius enim 77

nubere $\left(88^{*}\right)$

quam uri $\left.\left(\mathrm{PEL}^{\mathrm{B} *}\right)\right]$ qua[1] uiri $54^{*}$, lac. 64 , quam mori $\mathrm{PEL}^{\mathrm{var}}$, qua uri $\mathrm{PEL}^{\mathrm{BC}}$

7:10 Present: 51545861647576777888 89 AMst PEL TES SPM SPE

his autem $58(78)]$ iis autem $\mathrm{AMst}^{\mathrm{R}}$

qui TES var ] quae TES ${ }^{\text {ed }}$

matrimonio iuncti sunt $\mathrm{TES}^{\mathrm{var}}$ ] sunt in coniungio 64, in matrimonio iuncti sunt $88^{\mathrm{C}}$, matrimonio uincti sunt $\mathrm{AMst}^{\mathrm{var}}$, nupserunt $\mathrm{TES}^{\mathrm{ed}}$; + uel nubentibus 77

praecipio $51545861\langle 64\rangle 76(88)]$ denuntio $77 \mathrm{AMst}^{\mathrm{R}}$

non ego sed dominus

uxorem ] mulierem 64

a uiro non $\langle 64\rangle]$ uiro non TES ${ }^{\text {var }}$

discedere TES $\left.{ }^{\mathrm{var}}\right][\ldots]$ cedere 64, separari TES ${ }^{\mathrm{ed}}$, recedere $\mathrm{TES}^{\mathrm{var}} \mathrm{SPM}^{\mathrm{var}}$, discere $\mathrm{SPM}^{\mathrm{var}}$ 


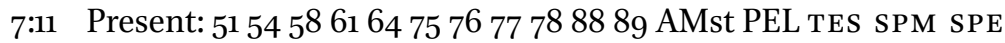
quod si ] si autem et 77 , si autem TES ${ }^{\text {ed }}$, sed et si TES ${ }^{\text {var }}$ discesserit TES $S^{\mathrm{var}}$ ] discederit 61 , recesserit $64 \mathrm{TES}^{\mathrm{ed}} \mathrm{SPM}^{\mathrm{var}}$ manere innuptam $\langle 64\rangle]$ manere innupta PEL ${ }^{\mathrm{var}} \mathrm{TES}^{\mathrm{var}}$ aut $]$ om. $54^{*}$, aut ce $[r]$ te TES ${ }^{\text {var }}$ uiro suo reconciliari AMst ${ }^{\text {var }}$ ] a uiro suo reconciliari $54^{*} 88$, uiro reconciliari $7576777889 \mathrm{AMst}^{\text {ed }} \mathrm{PEL}^{\mathrm{B}} \mathrm{TES}^{\mathrm{var}} \mathrm{SPM}^{\text {ed }}$, uiro reconciliare $\mathrm{AMst}^{\mathrm{AD} *}$, reconciliari uiro $\mathrm{TES} S^{\text {ed }}$, reconciliare a uiro $\mathrm{TES}^{\mathrm{var}}$, uiro reconcilietur $\mathrm{SPM}^{\mathrm{var}}$ et $\langle 64\rangle$ ] nam $\mathrm{AMst}^{\mathrm{AD} *}$, aut $\mathrm{TES}^{\mathrm{var}}$, ut $\mathrm{SPM}^{\mathrm{var}}$ uir uxorem $\langle 64\rangle \mathrm{AMst}^{\mathrm{var}} \mathrm{TES}^{\mathrm{var}}$ ] uirum uxorem $777^{8} \mathrm{AMst}^{\mathrm{ed}} \mathrm{PEL}^{\mathrm{B}} \mathrm{TES}^{\text {ed }}$ ne AMst ${ }^{\text {var }}$ TES $^{\text {var }}$ ] non $5^{1} 545^{8} 617778$ AMsted $^{\text {ed }}$ EL $^{\mathrm{B}}$ TES $^{\text {ed }}$ SPM $^{\text {var }}$ SPE $^{\text {ed, }}$, om. SPE ${ }^{\mathrm{var}}$

dimittat $\left.\mathrm{AMst}^{\mathrm{var}} \mathrm{TES}^{\text {var }}\right]$ dimittere $7778 \mathrm{AMst}^{\text {ed }} \mathrm{PEL}^{\mathrm{B}} \mathrm{TES}^{\mathrm{ed}}$, demittat $\mathrm{PEL}^{\mathrm{A}}$, remittere $\mathrm{TES}^{\mathrm{var}}$, dimitteret $\mathrm{TE} \mathrm{S}^{\mathrm{var}}$

7:12 Present: 51545861647576777888 89 AMst PEL CAr SPE

nam ceteris $6 r$ ] ceteris autem 6475767778 89, abs. CAr

ego dico ] lac. 64, abs. CAr

non dominus ] abs. CAr

si quis frater $\left.(78)^{\dagger}\right]$ si quis $\mathrm{PEL}^{\mathrm{B}}$

uxorem habet 54 ] habet uxorem $61\langle 64\rangle$, uxorem habeat AMstvar

infidelem ] [...] delem 64, non fidelem PEL ${ }^{\text {var }}$

et haec 51545876 ] et hac 78 , et hic 89, abs. CAr

consentit ] consen[...] 64, consensit 75, abs. CAr

habitare ] lac. 64, habere PEL var, abs. CAr

cum illo ] lac. 64, cum illa $89^{*}$, abs. CAr

non dimittat 5488 ] non demittat $61 \mathrm{PEL}^{\mathrm{A}}$, lac. 64, abs. CAr

illam ] lac. 64, eam PEL var, abs. CAr; + uir PEL var

7:13 Present: 51545861757677788889 AMst PEL $^{\dagger}$ SPE

et si qua mulier ] et mulier si qua 77 , et qua mulier $89^{*}$; + fidelis $P E L^{\text {var }}$

habet ] habeat AMst ${ }^{\mathrm{var}}$

uirum infidelem $\langle 76\rangle]$ uirum non fidelem $\mathrm{PEL}^{\mathrm{B}}$

et hic ] et haec PEL var

consentit habitare cum

illa non ] ea non $78 \mathrm{AMst}^{\mathrm{AD}}$

dimittat uirum $\mathrm{AMst}^{\mathrm{AD}}$ ] relinquat uirum 61 $\mathrm{AMst}^{\mathrm{ed}} \mathrm{PEL}^{\mathrm{B}}$, demittat uirum PEL

$\dagger{ }_{1}$ Cor. 7:12. In VL 78, the Greek tis has been written in place of quis.

$\dagger{ }_{1}$ Cor. 7:13. This verse is missing from three of the secondary manuscripts of PEL. 
7:14 Present: 515458617576777888 89 AMst PEL SPE

sanctificatus ] sanctificatur 78

est enim AMstrar $]$ est $61 \mathrm{AMst}^{\text {ed }}$, enim $75^{*} 78$, enim est $75^{\mathrm{C}}{ }^{6} 6$

uir infidelis $\left.\left\langle{ }_{76}\right\rangle\right]$ uir PEL ${ }^{\text {var }}$

in muliere fideli AMst ${ }^{\mathrm{AD}}$ ] per mulierem fidelem $5^{1} 545^{8} 61$, in uxore fidei

$75^{*}$, in uxore fideli $75^{\mathrm{C}} 7689 \mathrm{AMst}^{\mathrm{ed}} \mathrm{PEL}^{\mathrm{B}}$, muliere uel uxore fideli 77 , per uxorem fidelem $\mathrm{AMst}^{\mathrm{R}}$

et sanctificata ] et sanctificatus $77^{*}$

est mulier infidelis ] mulier fidelis AMst ${ }^{\text {var }}$

per uirum fidelem ] in fratre 75767778 , per maritum fidelem $89 \mathrm{PEL}^{\mathrm{B}}$, in uiro fideli $A M s t^{\text {ed }} S P E^{\text {ed }}$, a uiro fideli AMstrar, ex uiro fideli $A M s t^{\mathrm{R}}$, in uirum fidelem $\mathrm{SPE}^{\mathrm{var}}$

alioquin ] alioqui 77

filii uestri inmundi essent 7576 ] filii uestri inmundi sunt 7778

nunc autem ] nunc uero 78

sancti sunt ] sanctificati sunt 61

7:15 Present: 515458617576777888 89 AMst PEL SPE

quod si ] + autem 77

infidelis discedit ] infidelis discederit $\mathrm{AMst}^{\mathrm{AD}}$, infidelis descendit AMstrar discedat ] om. $\mathrm{PEL}^{\mathrm{B}}$

non est enim ] non est $75767778 \mathrm{AMst}^{\mathrm{AD}}$, non enim 88

seruituti $A M s t^{\mathrm{AD}}$ ] om. AMsted, seruitute PEL ${ }^{\mathrm{var}} \mathrm{SPE}^{\mathrm{var}}$

subiectus $\mathrm{AMst}^{\mathrm{AD}}$ ] deditus $61 \mathrm{PEL}^{\mathrm{B}}$, om. AMst $^{\mathrm{ed}}$

frater aut soror ] frater uel soror $77 \mathrm{AMst}^{\mathrm{AD}}$; + seruituti subiectus AMsted

in eiusmodi AMstvar ${ }^{\text {var }}$ in huiusmodi 5461 AMsted $^{\text {ed }}$, in talibus AMst ${ }^{\mathrm{AD}}$

in pace autem $\left(77^{*}\right)^{\mathrm{C}}$ ] in pacem autem 7576 , in pace enim 7889 , nam in pace PEL ${ }^{\text {var }}$; uel enim 77

uocauit nos ] uocauit uos PEL var

deus ] dominus 54

7:16 Present: 515458617576777888 89 AMst PEL SPE

unde enim scis mulier ] quid enim scis mulier 75767778 AMst

si uirum saluum facies $A M s^{A D R}$ ] si uirum saluum facias $\mathrm{AMst}^{\mathrm{ed}} \mathrm{PEL}^{\mathrm{var}}$

aut unde ] om. $54^{*}$, aut quid $7576777889 \mathrm{AMst}^{\mathrm{AD}}$

scis uir si ] om. $54^{*}$

mulierem AMst ${ }^{\mathrm{ADR}}$ ] om. 54*, uxorem 617576777889 AMst $^{\mathrm{ed}}$

saluam facies AMst $\left.{ }^{\mathrm{ADR}}\right] \mathrm{om} .54^{*}$, saluam facias AMst ${ }^{\text {ed }}$

7:17 Present: 515458617576777888 89 AMst PEL SPE

nisi ] om. $61 \mathrm{AMst}^{\mathrm{AD}}$ 
unicuique ] unumquemque PEL ${ }^{\text {var }}$, om. $\mathrm{PEL}^{\mathrm{var}}$; + autem 61, + et $\mathrm{AMst}^{\mathrm{R}}$ sicut ] om. PEL var

diuisit dominus ] deus diuisit $5^{8} \mathrm{AMst}^{\mathrm{var}} \mathrm{SPE}^{\mathrm{var}}$, dominus partitus est 61 $\mathrm{PEL}^{\mathrm{B}}$, diuisit deus $88 \mathrm{AMst}^{\mathrm{R}}$, om. PEL ${ }^{\text {var }}$; + et $547778 \mathrm{PEL}^{\mathrm{var}} \mathrm{SPE}^{\mathrm{var}}$ unumquemque 77 ] unumque 61 sicut uocauit ] sicut uocabit 75 deus $\mathrm{AMst}^{\mathrm{var}}$ ] dominus $61 \mathrm{AMst}^{\mathrm{ed}}$, dominus deus 77 ita ambulet ] sic ambulet $\mathrm{AMst} \mathrm{AD}^{\mathrm{AD}}$

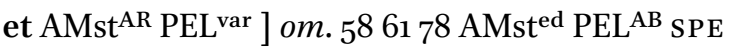
sic ] sicut $545^{86178} 88$ AMst PEL SP E, ita 75767789 in ] ubique in PEL $^{\mathrm{B}}$ omnibus ecclesiis $5^{1} 61$ ] ecclesiis omnibus 7576777889 AMst $^{\mathrm{A}}$, ecclesiis hominibus $\mathrm{AMst}^{\mathrm{D}}$, omnibus et ecclesiis $\mathrm{SPE}^{\mathrm{var}}$ doceo ] praecipio $A M s t^{A D}$

7:18 Present: $5^{1} 545^{8} 61757677788889$ AMst PEL sPE

circumcisus 61

aliquis ] qui 7576 , om. $\mathrm{SPE}^{\mathrm{var}}$

uocatus est ] uocatus 7778

non adducat praeputium in praeputio 51545861767778 (88)

aliquis $\mathrm{AMst}^{\mathrm{AD}}$ ] quis $\mathrm{AMst}^{\text {ed }} \mathrm{PEL}^{\mathrm{B}}$

uocatus est ] uocatus 7778

non ] om. $5^{8}$

circumcidatur ] circumdatur $5^{8 *}$

7:19 Present: $5^{1} 545^{8} 61647576777888$ 89 AMst PEL sPE circumcisio nihil est 515461 ] lac. 64 et ] lac. 64, om. 777889 praeputium nihil est 515458617688 ] lac. 64, om. 7778 sed ] lac. 64, om. PEL ${ }^{\mathrm{A}}$ obseruatio $5^{8}$ ] lac. 64, serua 7576 , om. PELA mandatorum $\langle 64\rangle$ ] mandatum 7576 , om. PEL $^{\mathrm{A}}$ dei ] om. PEL ${ }^{\mathrm{A}}$ \pm necessaria est $61 \mathrm{PEL}^{\mathrm{B}}$, bene facit 75

7:20 Present: 51545861647576777888 89 AMst PEL sPE unusquisque ] + autem PEL var in qua uocatione $\left.5^{8}\langle 64\rangle \mathrm{AMst}^{\mathrm{AD}}\right]$ in ea uocatione in qua $61 \mathrm{AMst}^{\mathrm{ed}}$, in uocatione qua 7778

uocatus est $\langle 64\rangle]$ uocati estis $\mathrm{AMst}^{\mathrm{var}}$ 
in ea $\mathrm{AMst}^{\text {var }}$ ] in ipsa $61757689 \mathrm{AMst}^{\text {ed }} \mathrm{PEL}^{\mathrm{B}}$

permaneat ] permanet $\mathrm{PEL}^{\mathrm{B}}$

\pm apud deum 6189 PEL $^{\mathrm{AB}}$

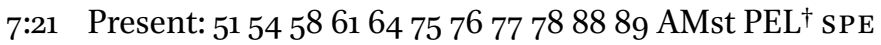
seruus uocatus es $\langle 64\rangle]$ seruus uocatus est $75^{*}$, om. PEL var non ] nihil $757689 \mathrm{AMst}^{\mathrm{AD}} \mathrm{PEL}^{\text {var }}$, om. PEL ${ }^{\text {var }}$

sit tibi curae 51545888 ] sit tibi cura $61 \mathrm{AMst}^{\mathrm{var}}$, ad te pertineat $64 \mathrm{AMst}^{\mathrm{AD}}$, cures 757689 , tibi sit curae $7778 \mathrm{PEL}^{\mathrm{var}}$, tibi cura PEL ${ }^{\mathrm{var}}$, om. PEL ${ }^{\mathrm{var}}$, sit sibi cura SPEvar

\pm de libertate 61, quod PEL var

sed et si AMst ${ }^{\text {var }}$ ] sed si 617778 AMst $^{\text {ed }}$, lac. 64

potes liber fieri ] [liber] potes fieri 64, potes fieri liber fieri $89^{*}$, potest liber fieri AMst ${ }^{\mathrm{var}}$, potes fieri liber PEL var magis utere 54 ] om. PEL var

7:22 Present: $51545^{8} 61647576777888$ 89 AMst PEL sPE qui enim in domino uocatus est $\langle 64\rangle]$ qui enim in domino est 89 seruus libertus

est domini ] est [...] 64, domini est 7778 , est dei $\mathrm{AMst}^{\mathrm{D}}$ similiter $\langle 64\rangle]$ + et $586189 \mathrm{AMst}^{\text {ed }} \mathrm{PEL}^{\mathrm{A}}$, + autem et 75767778 qui liber uocatus est seruus $\langle 64\rangle$ est christi $\langle 64\rangle]$ christi est 77 78, christi PEL ${ }^{\text {var }}$

7:23 Present: $5^{1} 545^{861} 647576777888$ 89 AMst PEL sPE pretio empti estis nolite fiere $51545^{8}\langle 64\rangle 7576^{*} 788889$ AMst PEL serui ] om. 61, lac. 64 hominum

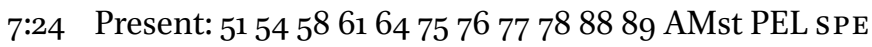
unusquisque ] om. PEL ${ }^{\mathrm{A}}$ \pm fratres $61647576777889 \mathrm{AMst}^{\mathrm{ed}}$, frater $\mathrm{AMst}^{\mathrm{var}} \mathrm{PEL}^{\mathrm{B}}$ in quo ] lac. 64, in $\mathrm{q}_{7}$, in qua uocatione $\mathrm{AMst}{ }^{\mathrm{AD}}$, om. $\mathrm{PEL}^{\mathrm{A}}$ uocatus est $\left.\langle 64\rangle\left(88^{*}\right)\right]$ om. PEL $^{\mathrm{A}}$

fratres ] frater $5^{1} 5^{\mathrm{C}} 88 \mathrm{sPE}$, fratres $54^{*}$, om. 61647576777889 AMst PEL in hoc ] in eo $6478 \mathrm{AMst}^{\mathrm{ed}}$, in ea $\mathrm{AMst}^{\mathrm{AD}}$, om. $\mathrm{PEL}^{\mathrm{A}}$

$\dagger{ }_{1}$ Cor. 7:21. In the Pseudo-Jerome tradition of PEL, this verse is followed by the text and exegesis of 1 Cor. 12:16. 
maneat ] permaneat $647888 \mathrm{SPE}$, permanent AMst ${ }^{\mathrm{var}}$, om. PEL $\mathrm{PL}^{\mathrm{A}}$ apud deum 5158757888 ] apud dominum 64, om. PEL ${ }^{\mathrm{A}}$

7:25 Present: $3^{2} 5^{1} 545^{8} 61647576777^{8} 84^{\dagger} 8889251$ AMst PEL CAr SPM SPE de ] e 54, lac. 64, fratres de 84251 uirginibus autem $\langle 64\rangle]$ uirginibus $3284 \mathrm{SPM}^{\text {var }}$, autem uirginibus $77 ;+$ ex $77^{*},+[6-10] 88^{*},+\operatorname{do}[2-3] 88^{\mathrm{C}}$

praeceptum domini $\left.\langle 32\rangle 5^{1} 545^{8} 61767778\langle\mathrm{CAr}\rangle\right]$ domini praeceptum $\mathrm{SPM}^{\mathrm{var}}$

non habeo $\langle 64\rangle$ ] lac. 32 , abs. 84

consilium autem ] consilio autem $\mathrm{SPE}^{\mathrm{var}}$, abs. $84 \mathrm{CAr}$

do ] abs. 84 CAr, datam AMstrar

tamquam 77 ] abs. 84 CAr, sicut AMst ${ }^{\mathrm{AD}}$ SPM, quam AMst ${ }^{\text {var }}$

misericordiam consecutus $\langle 64\rangle]$ abs. $84 \mathrm{CAr}$, consecutus misericordiam $\mathrm{PEL}^{\mathrm{B} *} ;+\operatorname{sum} 61^{\mathrm{C}} \mathrm{PEL}^{\mathrm{var}}$

a domino ] a deo $5^{1} 64 \mathrm{AMst}^{\mathrm{AD}}$, abs. $84 \mathrm{CAr}$, om. SPM, abs. CAr ut ] om. 77, abs. $84 \mathrm{CAr}$ sim fidelis ] fide[lis ...] 64, fidelis sim 7576 PEL $^{\text {var }}$, fidelis esse 77, abs. 84 CAr, fidelis essem $89 \mathrm{AMst}^{\mathrm{AD}} \mathrm{SPM}$

7:26 Present: $3^{2} 5^{1} 545^{8} 6164757677788889$ 251 AMst PEL SPM SPE existimo ergo ] ergo existimo 51 , existimo enim ergo $54^{*}$, existimo enim 58 617576 , [exi]stimo itaque 64, existimo ego 78, om. $\mathrm{PEL}^{\mathrm{B}}$

hoc bonum $\langle 32\rangle]$ bonum hoc 89 , bonum PEL ${ }^{\mathrm{A}} \mathrm{SPM}^{\mathrm{var}}$, om. $\mathrm{PEL}^{\mathrm{B}}$ esse propter ] lac. 64, propter $\mathrm{PEL}^{\mathrm{B}}$ instantem $\mathrm{AMst}^{\mathrm{AD}}$ ] lac. 64, presentem 89 AMst $^{\text {ed }} \mathrm{SPM}$ necessitatem $\langle 64\rangle$

quoniam $\langle 32\rangle$ ] lac. 64, quia 7576777889 AMst SPM, quam $\mathrm{PEL}^{\mathrm{B}}$ bonum est $\langle 32\rangle \mathrm{AMst}^{\mathrm{var}} \mathrm{SPM}^{\mathrm{var}}$ ] optimum est $61757689 \mathrm{AMst}^{\mathrm{ed}} \mathrm{SPM}^{\mathrm{ed}}$, lac. 64

homini sic esse 54 ] lac. 64, hominem sic esse 75 76, sic esse homini AMst ${ }^{\text {var }}$

7:27 Present: $3^{2} 5^{1} 545^{8} 61757677788889$ 251 AMst PEL SPE

alligatus $\mathrm{AMst}^{\mathrm{AD}}$ ] uinctus $61 \mathrm{AMst}^{\mathrm{ed}} \mathrm{PEL}^{\mathrm{B}}$

es uxori $\left(61^{*}\right)$ PEL $\left.{ }^{A C B C}\right]$ est uxori 75 PEL $^{A * B *}$

noli $\left.\langle 32\rangle \mathrm{AMst}^{\mathrm{AD}}\right]$ ne $77 \mathrm{AMst}^{\mathrm{ed}}$

$\dagger{ }_{1}$ Cor. 7:25. This verse appears twice in VL 84, once for St Agnes and once for St Euphemia. 
quaerere $51545^{8} 767888 \mathrm{AMst}^{\mathrm{AD}}$ ] quaere 77 , quae[...] 251, quaesieris $\mathrm{AMst}^{\mathrm{ed}}$, quereri SPEvar

solutionem $5889 *$ ] + uel quaerere resolutionem PEL ${ }^{\text {var }}$

solutus es ] solutus 6178 , solutus est 75

ab uxore $(75)(89)$

noli quaerere $\left.\left\langle 3^{2}\right\rangle 5^{1} 545^{8} 7688 \mathrm{AMst}^{\mathrm{AD}}\right]$ ne quaesieris $61 \mathrm{AMst}^{\text {ed }} P E L^{B}$, ne quaere 77 , ne quaeras 78

uxorem $\langle 251\rangle$ ] lac. 32

7:28 Present: $3^{2} 5^{1} 545^{8} 61757677788889$ 251 AMst PEL sPE

si autem ] si autem et 7778 , et si 89

acceperis uxorem ] acciperis uxorem $6189 \mathrm{SPE}^{\mathrm{var}}$, uxorem acceperis $\mathrm{AMst}{ }^{\mathrm{AD}}$ non peccasti et si $\left.\left\langle 3^{2}\right\rangle\right]$ non peccasti si AMst ${ }^{\text {var }}$

nupserit uirgo $326189\langle 251\rangle]$ nupserit uirga $\mathrm{PEL}^{\mathrm{A}}$

non peccauit $\mathrm{AMst}^{\mathrm{var}}$ ] non peccabit $32547888^{*} \mathrm{AMst}^{\mathrm{ed}} \mathrm{PEL}^{\mathrm{var}}$

tribulationem $\left.58 \mathrm{AMst}^{\mathrm{var}}\right]$ tribulationes $\mathrm{AMst}^{\mathrm{ed}}$

tamen ] autem 77

carnis habebunt $\mathrm{AMst}^{\mathrm{var}}$ ] habebunt carnis $61 \mathrm{AMst}^{\mathrm{ed}}$, in carne uel carnis habebunt 77

huiusmodi $\left.\left(\mathrm{PEL}^{\mathrm{A} *}\right)\right]$ om. 89

ego autem uobis parco 54

7:29 Present: $3^{2} 5^{1} 545^{8} 61757677788889$ 251 AMst PEL TES SPM SPE

hoc itaque dico $\mathrm{SPM}^{\mathrm{var}}$ ] hoc autem dico $7576777889 \mathrm{AMst}^{\mathrm{AD}} \mathrm{SPM}^{\mathrm{ed}}$, abs.

TES, hoc enim dico SPM ${ }^{\text {var }}$ + uel itaque 77

fratres ] abs. TES; + quia $757677,+$ qui $\mathrm{AMst}^{\mathrm{AD}}$

tempus ] + enim TES ${ }^{\text {var }}$

breue est (89) $\mathrm{AMst}^{\mathrm{AD}} \mathrm{TES}^{\mathrm{var}}$ ] breuiatum est $7576 \mathrm{AMst}^{\mathrm{ed}}$, breue uel breuiatum est 77 , collectum est $\mathrm{TES}^{\mathrm{ed}}$; + et $61 \mathrm{AMst}^{\mathrm{R}}$

reliquum est $546175 \mathrm{TES}^{\mathrm{var}}$ ] reliquum superest 88 , reliquum 89 , superest ergo TES ${ }^{\text {ed }}$

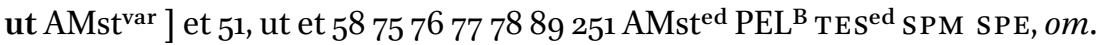
TESvar

qui habent ] + uel habentes 77

uxores ] maiores TES ${ }^{\text {var }}$

tamquam non habentes sint $5875^{*} \mathrm{AMst}^{\mathrm{AD}}$ TES $\left.^{\text {var }}\right]$ sint tamquam non habentes 61 , tamquam non habentes $7778 \mathrm{SPM}^{\mathrm{var}}$, ita sint ac si non habentes $\mathrm{AMst}^{\mathrm{ed}}$, ita sint ac si non habeant $\mathrm{PEL}^{\mathrm{B}}$, quasi non habentes sint $\mathrm{TE} \mathrm{S}^{\mathrm{ed}}$ 
7:30 Present: 3251545861757677788889 251 AMst PEL Tes SPM SPE et qui flent ] et qui flentes 7576 , et qui fluent $P_{E L}{ }^{A *}$, et qui plangent TES ${ }^{\text {var; }}$ + uel flentes 77

tamquam $75^{*} 77(88)$ TES $^{\text {var }}$ ] quasi TES $S^{\text {ed }}$

non flentes ] non plangentes TES ${ }^{\mathrm{var}}$; + sint $\mathrm{AMst}^{\mathrm{AD}} \mathrm{SPM}$

et qui gaudent ] et gaudentes 77

tamquam $\left.75^{*} 77 \mathrm{TES}^{\mathrm{var}}\right]$ quasi TES ${ }^{\text {ed }}$

non gaudentes ] + sint SPM

et qui emunt ] abs. SPE; + uel ementes 77

\pm tamquam non ementes et qui possident TES $S^{\text {var }}$

tamquam $75^{*} 77$ ] om. $\mathrm{PEL}^{\mathrm{B}}$, quasi TES, abs. SPE

non possidentes 61251 ] abs. SPE

7:31 Present: $3^{2} 5^{1} 545^{8} 61757677788889251$ AMst PEL TES SPM SPE et qui ] qui $\mathrm{PEL}^{\mathrm{B}}$

utuntur hoc mundo $54 \mathrm{AMst}^{\mathrm{ADR}}$ ] utuntur hunc mundum $75 \mathrm{SPE}^{\mathrm{var}}$, utuntur uel utentes mundum hunc 77 , utuntur huic mundo 78 , utantur de hoc mundo 89, utuntur hoc mundum 251, utuntur hoc saeculo AMsted, utitur hoc saeculo AMst ${ }^{\text {var }}$, hoc mundo utuntur TEs ${ }^{\text {ed }}$, utuntur de hoc mundo TES var

tamquam non $\left.75^{*} 77 \mathrm{TES}^{\mathrm{var}}\right]$ quasi non TES $S^{\text {ed }}$

utantur 54 ] abutentes uel abutantur 77 , utatur AMst ${ }^{\text {var }}$, utuntur PEL var SPEvar

praeterit 51545861777888 ] praeteriit $7576 \mathrm{TES}^{\mathrm{var}}$, praeteribit TES ${ }^{\mathrm{var}}$ enim ] om. TES var

figura $\mathrm{SPM}^{\mathrm{var}}$ ] habitus $7576 \mathrm{SPM}^{\mathrm{ed}}$, figura habitus 89, fuga $\mathrm{TES} \mathrm{S}^{\mathrm{var}}$, scisma TES ${ }^{\text {var }}$

huius mundi ] mundi huius 77

7:32 Present: 3251545861757677788889 251 AMst PEL TES SPM SPE uolo autem uos $\mathrm{AMst}^{\mathrm{AD}}$ ] uos autem uos 51, uos autem uolo 61, uolo uos 75 76777889 AMst $^{\text {ed }}$ PEL $^{\mathrm{B}}$ SPM, abs. TES

sine sollicitudine esse $\left.545861\left(88^{*}\right)\right]$ abs. TES

qui $88 \mathrm{TES}^{\mathrm{var}}$ ] si quis $88^{\mathrm{alt}}$, om. TES $\mathrm{S}^{\mathrm{ed}} \mathrm{SPM}^{\mathrm{var}}$, quia qui $\mathrm{SPE}^{\mathrm{var}}$, quia $\mathrm{SPE}^{\mathrm{var}}$ sine uxore est $32(76)\left(88^{*}\right)$ TES $\left.^{\text {var }}\right]$ caelebs TES ${ }^{\text {ed }}$, om. SPM ${ }^{\text {var }}$

sollicitus est 586178 ] cogitat AMst ${ }^{\text {ed }} \mathrm{PEL}^{\mathrm{A}}$ TES, cogitet AMstrar, om. SPM $^{\mathrm{var}}$ quae 515458617678 TES $\left.^{\text {var }}\right]$ ea quae TES ${ }^{\text {ed }}$

domini sunt ] sunt huius mundi $61^{*}$, sunt dei $61^{\mathrm{C}} 7889 \mathrm{TES}$ var, sunt domini $757677 \mathrm{TES}^{\mathrm{ed}}$; + et cogitat 54 , + et AMst ${ }^{\mathrm{var}}$

quomodo placeat deo $\mathrm{AMst}^{\mathrm{var}}$ ] quomodo placeat domino 7576 , om. AMsted SPM $^{\mathrm{var}}$ 
7:33 Present: $3^{2} 51545^{8} 61757677788889$ 251 AMst PEL tes SPM SPE qui autem ] quae autem TEs ${ }^{\mathrm{var}}$

cum uxore est $\mathrm{TES}^{\mathrm{var}}$ ] nubit uel habet uxorem 77 , matrimonio iunctus est 78 $\mathrm{TES}^{\mathrm{var}}$, uxorem habet $\mathrm{AMst}^{\mathrm{AD}} \mathrm{SPM}$, matrimonium contraxit $\mathrm{TES}^{\mathrm{ed}}$, matrimonium coniunxit TES ${ }^{\mathrm{var}}$

sollicitus est $586{ }^{8} 78$ ] cogitat 7576 TES SPM, sollicitat $777^{88^{\mathrm{gl}}}$

quae sunt 51545861767888 ] ea quae sunt TES ${ }^{\text {ed }}$

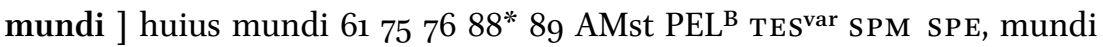
huius TES ${ }^{\text {ed }}$

\section{quomodo placeat uxori $\left(88^{*}\right)$}

7:34 Present: 3251545861757677788889251 AMst PEL TES SPM SPE et $\mathrm{AMst}^{\mathrm{var}} \mathrm{PEL}^{\mathrm{var}} \mathrm{TES}^{\mathrm{var}}$ ] om. $3^{2} 6175767789 \mathrm{AMst}^{\text {ed }} \mathrm{PEL}^{\mathrm{AB}} \mathrm{TES}^{\text {ed }} \mathrm{SPM}$ diuisus est $\mathrm{PEL}{ }^{\text {var }}$ ] om. $32 \mathrm{TES}^{\text {ed }}$, diuisa est $6775767789 \mathrm{AMst}^{\text {ed }} \mathrm{PEL}^{\mathrm{AB}} \mathrm{TES}^{\text {var }}$ $\mathrm{TES}^{\mathrm{var}} \mathrm{SPM}^{\mathrm{var}}$, diuisa 78 , diuisus est diuisa est $\mathrm{AMst}^{\mathrm{var}}$, separata est $\mathrm{SPM}^{\mathrm{ed}}$; + sicut TES $\mathrm{S}^{\mathrm{var}},+$ sic TES $\mathrm{S}^{\mathrm{ed}}$

et AMstvar PEL ${ }^{\text {var }}$ ] om. ${ }^{1} 1617889$ AMst $^{\text {ed }}$ PEL $^{\mathrm{AB}}$ TES $^{\text {var }}$ SPM

mulier innupta et uirgo $251 \mathrm{AMst}^{\mathrm{var}} \mathrm{PEL}^{\text {var }}$ ] uirgo quae innupta est 32 , mulier et uirgo $5_{1} \mathrm{PEL}^{\text {var }}$, mulier et uirgo quae non est nupta $61 \mathrm{SPM}^{\mathrm{ed}}$, mulier et uiro quae innupta est $75^{*} 89$, mulier et uirgo quae innupta est $75^{\mathrm{C}} 76 P E L^{A} \mathrm{SPM}^{\mathrm{var}}$, mulier et uirgo quae non est nupta uel innupta 77 , mulier et uirgo inmaculata $78^{*}$, mulier innupta et uirgo inmaculata $78^{\mathrm{C}}$, mulier et uirgo quae autem non est nupta AMsted, mulier et uirgo quae nupta est $\mathrm{AMst}^{\mathrm{AD}}$, mulier et uirgo innupta $\mathrm{PEL}^{\mathrm{B}} \mathrm{TES}^{\mathrm{ed}}$, mulier et uir ut innupta TES $S^{\mathrm{var}}$

cogitat AMst ${ }^{\mathrm{var}}$ ] cogitat uel sollicitat 77, sollicita est AMsted

\pm quae est nupta cogitat $\mathrm{AMst}^{\mathrm{A}}$, quae est nupta sollicita est $\mathrm{AMst}^{\mathrm{D}}$, quae sunt domini nam quae est nupta sollicita est $\mathrm{AMst}^{\mathrm{var}}$

quae $51545^{8} 61767888$ ] ea quae $77 \mathrm{TES}$, de his quae AMsted, de iis quae $\mathrm{AMst}^{\mathrm{R}}$

domini sunt (32) ] sunt domini 6175767778 PEL $^{\mathrm{B}}$ TES, dei sunt SPM ut sit sancta et PEL ${ }^{\text {var }}$ ] ut sit sancta 5161251 PEL $^{\mathrm{AB}} \mathrm{TES}^{\mathrm{var}}$ SPM $^{\mathrm{var}}$ corpore et spiritu

quae autem $51545^{8} 61767778 \mathrm{AMst}^{\mathrm{AD}}$ ] nam quae $\mathrm{AMst}^{\mathrm{ed}}$, quae uero $\mathrm{AMst}^{\mathrm{R}}$

nupta est 89251 TES $^{\mathrm{var}}$ ] innupta est $88^{*}$, nupsit TES ${ }^{\text {ed }}$, nubit TES $S^{\text {var }}$ cogitat ] om. 75 , cogita[1]t $89^{*}$; + de his TE sed $^{\text {ed }}$

quae sunt 515458617688 ] om. 75

mundi $\left.\langle 76\rangle \mathrm{TES}^{\mathrm{var}} \mathrm{SPM}^{\mathrm{var}}\right]$ om. 75 , huius saeculi 89, mundi huius AMstvar TES $S^{\text {ed }}$, huius mundi AMsted $\mathrm{PEL}^{\mathrm{B}} \mathrm{SPM}^{\mathrm{ed}}$, saeculi huius $\mathrm{AMst} \mathrm{AD}^{\mathrm{AD}}$ 
quomodo placeat ] om. 75

uiro ] om. $54^{*} 75$, uiro suo $58 \mathrm{SPE}$ var

7:35 Present: $3^{2} 51545861757677788889251$ AMst PEL SPE

porro hoc $\mathrm{AMst} \mathrm{var}^{\mathrm{var}}$ ] hoc $6175^{*} 89 \mathrm{PEL}^{\mathrm{B}}$, hoc autem ${ }_{75}{ }^{\mathrm{C}} 76777^{8} \mathrm{AMst}^{\mathrm{ed}}$

ad utilitatem uestram ] ad uestram utilitatem $75767778 \mathrm{AMst}^{\mathrm{AD}}$, ad uestram ipsorum utilitatem AMst ${ }^{\text {ed }}$

dico non ut laqueum 88

uobis iniciam 546777 ] iniciam uobis 89 PEL $^{B}$

sed ad id quod honestum est 88 ] sed ad honestum 77 , sed ut id quod honestum est $\mathrm{AMst}^{\mathrm{R}}$

et quod $\mathrm{AMst}^{\mathrm{R}} \mathrm{PEL} \mathrm{Lar}^{\mathrm{var}}$ om. $32 \mathrm{AMst}^{\mathrm{ed}} \mathrm{PEL} \mathrm{LB}^{\mathrm{AB}}$, ut sit 61 , et $757^{6} 78$, uel sed ad id quod 77 , ad 89

facultatem praebeat $\left.51545888 \mathrm{AMst}^{\mathrm{R}} \mathrm{PEL}^{\mathrm{var}}\right]$ om. $3^{2} 78 \mathrm{AMst}^{\mathrm{ed}} \mathrm{PEL}^{\mathrm{AB}}$, frequens oratio uestra 61 , praesente 7576 , honestum est et 77 , facilitatem praebeat $88^{a l t}$, praestolandum 89 , honestum $\mathrm{AMst}^{\mathrm{AD}}$

sine inpedimento $\left.5158 \mathrm{AMst}^{R} \mathrm{PEL}^{\mathrm{var}}\right]$ om. $3^{2} 61757689 \mathrm{AMst}^{\mathrm{ed}} \mathrm{PEL}^{\mathrm{AB}}$, bene stabile 7778

dominum AMst ${ }^{\mathrm{R}}$ ] om. $32 \mathrm{AMst}^{\mathrm{ed}} \mathrm{PEL}^{\mathrm{AB}}$, domino 5475767778 89 PEL $^{\text {var, }}$ ad dominum 61, christo $\mathrm{AMst}^{\mathrm{A}}$

obseruandi $\left.\langle 58\rangle \mathrm{PEL}^{\text {var }}\right]$ om. $3^{2} \mathrm{AMst}^{\mathrm{ed}} \mathrm{PEL}^{\mathrm{AB}}$, obsecrandi $512{ }^{1} \mathrm{AMst}^{\mathrm{R}}$ $\mathrm{SPE}^{\mathrm{var}}$, consecrandi 54 , sine ulla occupatione 61 , non recedentes 7576 , inadducibiliter 7778 , intente 89

7:36 Present: $3^{2} 51545^{8} 61757677788889$ 251 AMst PEL SPE

si quis autem ] quod si quis 7576 , si autem quis $777^{8}$

turpem se ] se turpem $\mathrm{AMst}^{\mathrm{var}}$, turpe $\mathrm{PEL}^{\mathrm{B}}$, turpem $\mathrm{PEL}^{\mathrm{var}}$

uideri existimat ] existimat $75767778 \mathrm{PEL}^{\mathrm{B}}$, uideri $[t]$ existimat $89^{*}$, uideri aestimat $\mathrm{AMst}^{\mathrm{var}}$, uiderit $\mathrm{AMst} \mathrm{t}^{\mathrm{ed}}$, existimat uidere $\mathrm{AMst}^{\mathrm{AD}}$, uidere existimat PEL ${ }^{\text {var }}$ + uideri 58

super $\mathrm{AMst}^{\mathrm{var}}$ ] in $757689^{*} \mathrm{AMst}^{\mathrm{AD}}$, super uel circa 77 , supra $\mathrm{AMst} \mathrm{t}^{\mathrm{ed}}$ PEL $^{\mathrm{A}}$

uirgine sua $\mathrm{AMst}^{\mathrm{A}} \mathrm{SPE}^{\mathrm{var}}$ ] uirginem suam 6175767788 251 $\mathrm{AMst}^{\text {ed }} \mathrm{PEL}^{\mathrm{B}}$ $S P E^{\text {ed }}$, uirginitatem suam $89^{*}$

quod ] et quod 51, si $75767789^{*} \mathrm{AMst}^{\mathrm{ed}}$, eo quod $89^{\mathrm{C} 2}$, qui $\mathrm{AMst}^{\mathrm{D} *}$, quia $\mathrm{AMst}^{\mathrm{D}}$

sit superadulta ] supra quam matura sit $757^{6}\left(89^{*}\right)^{\mathrm{C} 1}$, sit adulta $89^{\mathrm{C} 2}$, sit ultra pubertatem $\mathrm{AMs} \mathrm{t}^{\mathrm{ed}}$, supramatura sit $\mathrm{AMst}{ }^{\mathrm{AD}}$

et ita oportet fieri $\mathrm{AMst}^{\mathrm{AD}}$ ] et sic oportet fieri $\mathrm{AMst}^{\mathrm{ed}}$

quod uult faciat $\left(\mathrm{PEL}^{\mathrm{A}}\right)^{\mathrm{C}}$ ] quod faciat uult $\mathrm{AMst}^{\mathrm{var}}$ 
non peccat $\left.(54) \mathrm{PEL}^{\text {var }}\right]+\operatorname{si}^{2} 5^{1} 54^{\mathrm{C}} 5^{8} 61757_{7} 68889251$ AMst PEL $^{\mathrm{AB}}$ SPEvar

nubat ] nubet $\mathrm{PEL}^{\mathrm{B}}$

7:37 Present: $3^{2} 5^{1} 545^{8} 61757677788889251$ AMst PEL SPE

nam qui $A M s t^{\mathrm{R}}$ ] nam quis $54^{*}$, qui autem $7576777889 \mathrm{AMst}^{\mathrm{ed}}$

statuit in corde suo $\left(54^{*}\right)$

firmus AMst'var ] om. 7576777889 AMst $^{\text {ed }} \mathrm{PEL}^{\mathrm{B}}$, stabilis $\mathrm{AMst}^{\mathrm{AD}}$; + est 61

non ] om. PEL ${ }^{\mathrm{B}}$

habens necessitatem potestatem (88)

autem $\mathrm{AMst}^{\mathrm{A}}$ ] om. $88^{*} \mathrm{AMst}^{\mathrm{ed}} \mathrm{PEL} \mathrm{L}^{\text {var }}$

habet ] habens $5^{1} 5^{8} 7889^{\mathrm{C} 2} 25^{1} \mathrm{PEL}^{\mathrm{var}}$, habe[12] 88*, om. PEL var

suae uoluntatis 515458757688 ] de sua uoluntate 77 AMst $^{\text {ed }}$, propriae uoluntatis 78 , uoluntatis suae $\mathrm{AMst} \mathrm{AD}^{\mathrm{AD}}$

et hoc iudicauit ] et hoc statuit (75) $76777889 \mathrm{AMst}^{\mathrm{AD}}$, et hoc decreuit AMsted $^{\text {ed }}$

in corde suo ] in suo corde AMstvar

seruare ] ut seruet 757689 AMst $^{\mathrm{AD}}$

uirginem suam ] suam uirginem 7778 , uirginitatem suam $89 *$ AMst $^{\text {var }}$

bene facit ] om. 75 , bene faciat $78^{*}$

7:38 Present: $3^{2} 5^{1} 545^{8} 61757677788889$ 251 AMst PEL SPM SPE

igitur et $\mathrm{SPM}^{\mathrm{var}}$ ] itaque et $757689 \mathrm{AMst}_{\mathrm{SPM}}^{\mathrm{ed}}$, om. $777888^{*} \mathrm{SPM}^{\mathrm{var}}$

qui matrimonio iungit $\left(88^{\mathrm{C}}\right) \mathrm{SPM}^{\mathrm{var}}$ ] qui nupto dat 7576 , om. $777888^{*}$, qui

nuptum dat $89 \mathrm{AMst}^{\mathrm{ed}} \mathrm{SPM}^{\mathrm{ed}}$, qui dat nuptum $\mathrm{AMst}^{\mathrm{var}} \mathrm{SPM}^{\mathrm{var}}$

uirginem suam bene facit ] om. $777888^{*}$, uirginem suam $88^{\mathrm{C}}$

et qui non ] qui non $5^{8}$

iungit $61 \mathrm{SPM}^{\text {var }}$ ] dat nupto 7576 , dat nuptum $777889 \mathrm{AMst}^{\mathrm{ed}}$, dat $\mathrm{AMst}^{\mathrm{var}}$ $\mathrm{SPM}^{\mathrm{ed}}$

melius facit

7:39 Present: $5^{1} 545^{8} 61757677788889251$ AMst PEL TES SPE

mulier AMst ${ }^{\text {var }}$ ] uxor AMst ${ }^{\text {ed }}$

alligata est $A M s^{\text {var }}$ TES $^{\text {var }}$ ] uincta est $61 \mathrm{AMst}^{\text {ed }} \mathrm{PEL}^{\mathrm{var}(\mathrm{ed})} \mathrm{TES}^{\mathrm{ed}}$, uincta uel alligata est 77 , iuncta est $P E L^{\mathrm{B}}$, uicta est $\mathrm{TES}^{\mathrm{var}}$

\pm legi $5^{1} 545^{861} 777888251$ AMst $^{\text {var }}$ PEL $^{\mathrm{B}} \mathrm{TES}^{\mathrm{var}} \mathrm{SPE}^{\mathrm{var}}$, lege AMst ${ }^{\text {ed }}$

quanto tempore ] quamdiu 89 AMst TES; + uel quamdiu $77,+$ iuncta est legi $\mathrm{PEL}^{\mathrm{B} *}$

uir eius uiuit $\mathrm{AMst} \mathrm{AD}^{\mathrm{AD}}$ ] uiuit uir eius $6175767778 \mathrm{AMst}^{\mathrm{ed}} \mathrm{TES}$

quod si AMst ${ }^{\mathrm{ADR}}$ ] om. $76^{*}$, si autem 7778 AMst $\mathrm{Ad}^{\mathrm{ed}} \mathrm{TES}$ 
dormierit uir eius $\left.A M s t^{\mathrm{R}}\right]$ dormitionem uir eius acciperit $\left\langle{ }_{75}\right\rangle\left\langle 7^{\mathrm{C}^{*}}\right\rangle$, om. $76^{*}$, dormitionem acceperit uir $89^{*}$, mortuus fuerit uir eius $\mathrm{AMst}^{\mathrm{ed}}$, dormitionem acciperit uir eius $\mathrm{AMst}^{\mathrm{AD}}$, dormierit $\mathrm{TES}^{\mathrm{ed}}$, mortuus fuerit TES $^{\text {var }}$, dormiuerit uir eius TES ${ }^{\text {var }}$

liberata est ] libera est $\mathrm{AMst}^{\mathrm{ADR}}$, libera facta est TE $\mathrm{S}^{\mathrm{var}}$

\pm a lege uiri $51545^{8} \mathrm{TES}^{\mathrm{var}}$, a lege $25^{1}$

cui ] ut cui TES ${ }^{\text {var }}$

uult (75) TES ${ }^{\text {var }}$ ] uolet TES ${ }^{\text {ed }}$, uoluerit TES ${ }^{\text {var }}$

nubat tantum in domino

7:40 Present: 51545861757677788889 251 AMst PEL TES SPE

beatior TES ${ }^{\text {var }}$ ] felicior TES $S^{\text {ed }}$

autem erit ] autem est 757677 78, est autem 89, erit autem PEL var

si sic ] qui sic 58 , sic sic $88^{*}$ PEL $^{\mathrm{A}}$

permanserit ] manserit TES ${ }^{\mathrm{var}}$

secundum meum consilium puto (51) ] abs. TES

autem ] enim $61 \mathrm{AMst}^{\mathrm{ADR}}$, om. $75^{*} \mathrm{PEL}^{\mathrm{B}}$, abs. TES

quod et ego AMst ${ }^{\text {var }}$ ] et ego autem $75^{*}$, et ego $75^{\mathrm{C}} 767789^{*} \mathrm{AMst}^{\text {ed }} \mathrm{PEL}^{\mathrm{B}}$, et me AMst ${ }^{\mathrm{R}}$, et ego quod PEL ${ }^{\text {var }}$, ego quod PEL ${ }^{\mathrm{var}}$, abs. TES

spiritum dei ] abs. TES

habeo $S E^{\text {var }}$ ] habeam $5^{1} 545^{8} 617825^{1} \mathrm{AMst}^{\mathrm{var}} \mathrm{PEL}^{\mathrm{var}} \mathrm{SPE}{ }^{\mathrm{ed}}$, habere $75^{\mathrm{C}} 7^{6}$ $\mathrm{AMst}^{\mathrm{R}}$, abs. TES; + in christo iesu domino nostro 251

8:1 Present: $5^{1} 545^{8} 61757677788889$ AMst PEL CAr SPE

de his autem quae idolis 51545861767888

sacrificantur $\mathrm{AMst} \mathrm{AD}^{\mathrm{AD}}$ ] immolantur $586178 \mathrm{AMst}^{\mathrm{ed}} \mathrm{PEL}^{\mathrm{B}} \mathrm{CAr}$, immolantur uel sacrificantur 77

scimus quia ] scimus quoniam $5^{1} 75767788 \mathrm{PEL}^{\mathrm{A}}$, scimus quod PEL ${ }^{\mathrm{var}}$, abs. CAr

omnes ] omnem SPE ${ }^{\mathrm{var}}$, abs. CAr

scientiam 5888 ] abs. CAr; + huius modi PEL ${ }^{\text {var }}$, + huius mundi PELvar

habemus scientia 545888 ] abs. CAr; + autem $54^{\mathrm{C}} 61$, + enim $\mathrm{PEL}^{\mathrm{B}}$

inflat caritas ] abs. CAr

uero ] autem $61^{*} 7576$, uero uel autem 77, om. 78 , abs. CAr

aedificat 5154587688 ] abs. CAr

8:2 Present: $51545^{8} 61757677788889$ AMst PEL TES SPE

si quis TES ${ }^{\mathrm{var}}$ ] si quis autem $5^{1} 5^{8} 7888 \mathrm{SPE}^{\mathrm{var}}$, quod si quis $617576 \mathrm{AMst} \mathrm{t}^{\mathrm{ed}}$ $\mathrm{PEL}^{\mathrm{B}}$, si autem uel quod si quis 77, si quis enim 89, sed si quis AMst ${ }^{\mathrm{var}}$, si qui TES ${ }^{\text {ed }}$ 
se existimat scire aliquid 54 ] existimat se scire aliquid 58 , se existimat cognouisse aliquid $7576 \mathrm{AMst}^{\mathrm{D}}$, se existimat cognoscere uel scire aliquid 77 , putat se scire aliquid 78 , existimat cognouisse se aliquid 89 , existimat se scire AMst ${ }^{\text {ed }}$, cognouisse se existimat aliquid $\mathrm{AMst}^{\mathrm{A}}$, existimat se aliquid scire $A M s t^{\mathrm{R}}$, se existimet scire aliquid $P E L^{v a r}$, se putat scire aliquid TES ${ }^{\text {ed }}$, putat scire aliquid TES ${ }^{\mathrm{var}}$, se scire aliquid existimat $\mathrm{SPE}^{\mathrm{var}}$; + in uobis TES $S^{\text {var }}$

nondum ] nos dum hoc TES var

cognouit quemadmodum $\mathrm{TES}^{\mathrm{var}}$ ] quemadmodum scit TES $\mathrm{S}^{\mathrm{ed}}$

oporteat ] oportet $61 \mathrm{PEL}^{\mathrm{B}}$, debeat TES ${ }^{\mathrm{var}}$

eum scire TES ${ }^{\text {var }}$ ] scire 75767778 89* AMst TES ${ }^{\text {ed }}$, eum PEL ${ }^{\text {var }}$

8:3 Present: 51545861757677788889 AMst PEL SPE

si quis autem $\mathrm{AMst}^{\mathrm{var}}$ ] si autem quis 7778 , si qui autem $88^{*}$, si uero quis AMst $^{\text {ed }}$

diligit deum hic cognitus est 61

ab eo ] ab illo $75767778 \mathrm{AMst}^{\mathrm{AD}}$, a deo 89

8:4 Present: $5^{1} 545^{8} 6175767778848889$ AMst PEL SPM SPE

de ] fratres de 84

escis (61) $76 \mathrm{AMst}^{\mathrm{AD}}$ ] esca 7778 , cibis $\mathrm{AMst}^{\mathrm{ed}}$

autem $\mathrm{AMst}^{\mathrm{var}} \mathrm{SPM}^{\mathrm{var}}$ ] ergo $7778 \mathrm{SPM}^{\mathrm{ed}}$, om. 84 , igitur $\mathrm{AMst}^{\mathrm{ed}}$

quae idolis immolantur $5154586188 \mathrm{AMst}^{\mathrm{var}} \mathrm{SPM}^{\mathrm{var}}$ ] sacrificiorum 757677

$7889 \mathrm{AMst}^{\mathrm{AD}} \mathrm{SPM}^{\mathrm{var}}$, simulacris immolatorum AMsted, eorum quae idolis immolantur $\mathrm{PEL}^{\mathrm{B}}$, sacrificiorum quae idolis immolantur PEL ${ }^{\text {var, }}$, sacrificatorum $\mathrm{SPM}^{\mathrm{ed}}$

scimus ] abs. 84; + autem $51,+$ enim AMst $^{\text {ed }}$

quia nihil est 5154 ] quia nihil 7576 , abs. 84

idolum 51586 AMst $^{\text {var }}$ ] abs. 84, simulacrum AMst ${ }^{\text {ed }}$

in mundo PEL var $\mathrm{SPM}^{\mathrm{var}}$ ] om. $6189 \mathrm{AMst}^{\mathrm{ed}} \mathrm{PEL}^{\mathrm{AB}} \mathrm{SPM}^{\mathrm{ed}}$, in saeculo 7576 , abs. 84, in hoc mundo AMst ${ }^{\mathrm{AD}}$, in mundum $\mathrm{SPE}^{\mathrm{var}}$

et quod AMst ${ }^{\text {var }}$ ] eo quod 54, et quia $78 \mathrm{AMst}^{\text {ed }}$ PEL ${ }^{\text {var }}$ SPM, abs. $84 \mathrm{SPE}$ nullus AMst ${ }^{\mathrm{var}}$ ] nemo $757689^{*}$ AMst $^{\text {ed }}$ SPM, abs. 84 SPE, non est $89^{\mathrm{C} 2}$ deus nisi unus ] abs. $84 \mathrm{SPE}$; deus $61 \mathrm{SPM}^{\mathrm{var}}$

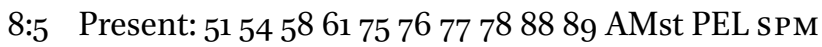

nam et si ] etenim si 78 , nam et $88^{*}$, licet $\mathrm{AMst}^{\mathrm{ed}}$, nam etsi $\mathrm{AMst}^{\mathrm{AD}} \mathrm{SPM}$

sunt qui ] sint qui $757689 \mathrm{AMst}$, om. SPM ${ }^{\mathrm{var}}$

dicantur ] dicuntur $61 \mathrm{AMst}^{\mathrm{AD}} \mathrm{PEL}^{\mathrm{B}} \mathrm{SPM}^{\mathrm{var}}$

dii $\mathrm{AMst}^{\mathrm{AD} *}$ ] dei SPM ${ }^{\mathrm{var}}$; + et domini $7576 \mathrm{AMst}^{\mathrm{ed}}$ 
siue in caelo 515458767788$]+$ si $88^{*}$

siue in terra

siquidem $\mathrm{AMst}^{\mathrm{R}}$ ] sicut enim 7576 , sicut $7778 \mathrm{AMst}^{\mathrm{AD}}$, sicuti 89, om. AMst $\mathrm{t}^{\mathrm{ed}}$ $\mathrm{PEL}^{\mathrm{B}}, a b s$. SPM

sunt dii multi et domini multi $\left.75 \mathrm{AMst}^{\mathrm{ADR}}\right]$ om. AMst ${ }^{\mathrm{ed}} \mathrm{PEL}^{\mathrm{B}}$, abs. SPM

8:6 Present: $5^{1} 545^{8} 61757677788889$ AMst PEL sPM

nobis tamen ] nobis autem $54 \mathrm{AMst}^{\mathrm{AD}} \mathrm{PEL}^{\mathrm{B}}$, sed nobis 75767778 , nobis 89 unus ] + est 757689 sPM $^{\text {ed }}$

deus pater ex quo omnia $\left.S \mathrm{PM}^{\mathrm{var}}\right]+$ nobis $S \mathrm{PM}^{\mathrm{ed}}$

et nos in ] et nos per 89

illum ] illo $5^{1} 5^{8} \mathrm{AMst}^{\text {ed }} \mathrm{PEL}^{\mathrm{A}}$, ipsum $757689 \mathrm{AMst}^{\mathrm{var}} \mathrm{SPM}^{\mathrm{ed}}$, ipsum uel ipso 77 , ipso $78 \mathrm{AMst}^{\mathrm{D}} \mathrm{SPM}^{\mathrm{var}}$

et unus ] et unus est 61

dominus iesus christus $\mathrm{AMst}{ }^{\mathrm{AD}}$ ] dominus iesus $75 * 78 \mathrm{AMst}^{\mathrm{ed}} \mathrm{PEL}^{\mathrm{B}}$

per quem omnia et nos

per ipsum ] in ipso $89 \mathrm{AMst}^{\mathrm{D}}$, in ipsum $\mathrm{AMst}^{\mathrm{var}}$

8:7 Present: $51545^{86175767778} 88$ 89 AMst PEL sPE

sed non in omnibus est ] sed non in omnibus 7576 , sed non est in omnibus SPE ${ }^{\mathrm{var}}$

scientia $5458\langle 61\rangle 88$

quidam autem $\mathrm{AMst}^{\mathrm{AD}}$ ] quidam enim 89 , aliqui autem $\mathrm{AMst}^{\mathrm{ed}}$, nam aliqui $\mathrm{AMst}^{\mathrm{R}}$

conscientia $54^{*} 58$ ] conscientiam hunc $54^{c}$, cum scientia 6188 , in conscientia $75767778 \mathrm{PEL}^{\mathrm{A}}$, in conscientiam 89, om. AMst ${ }^{\mathrm{ed}}$, consuetudine $\mathrm{AMst}^{\mathrm{AD}}$, cum conscientia sua PEL ${ }^{\text {var }}$

usque nunc ] adhuc 61, usque adhuc $7576777889 \mathrm{PEL}^{\mathrm{B}}$, idoli usque adhuc $\mathrm{AMst}^{\mathrm{AD}}$, adhuc conscientia $\mathrm{AMst}^{\mathrm{ed}}$, adhuc scientia $\mathrm{AMst}^{\mathrm{var}}$

idoli $5154 \mathrm{AMst}^{\mathrm{R}}$ ] idoli hunc 58 , simulacri $\mathrm{AMst}{ }^{\mathrm{ed}}$, om. AMst ${ }^{\mathrm{AD}} \mathrm{PEL}^{\mathrm{B}}$, idolo $\mathrm{PEL}^{\mathrm{var}} \mathrm{SPE}^{\mathrm{var}}$

quasi ] qua $\mathrm{AMst}{ }^{\mathrm{var}}$, om. $\mathrm{AMst}^{\mathrm{AD}}$

idolothytum $5^{1} 54\langle 61\rangle 7888$ ] idolaticum 58 , idolo immolatum 757689

$\mathrm{AMst}^{\mathrm{D}}$, simulacro immolatum AMst ${ }^{\mathrm{ed}}$, idolum immolatum $\mathrm{AMst}^{\mathrm{A}}$; + uel idolo immolatum 77

manducant ] + uel edunt 77

et conscientia 58

ipsorum ] eorum $7576777888^{*} 89$ AMst $^{\mathrm{AD}} \mathrm{PEL}^{\text {var }}$, aliorum $\mathrm{AMst}^{\mathrm{ed}}$, illorum AMst $^{\text {var }}$

cum sit infirma ] cum adhuc sit infirma AMst ${ }^{\text {var }}$ 
polluitur $\left(88^{*}\right) \mathrm{AMst}^{\mathrm{D}}$ ] inquinatur $6175767789 \mathrm{PEL}^{\mathrm{B}}$, inquinabitur 78 , coinquinatur $\mathrm{AMst}^{\mathrm{ed}}$, contaminatur $\mathrm{AMst}^{\mathrm{AD} *}$, coinquinabitur $\mathrm{AMst}^{\mathrm{var}}$

8:8 Present: 515458617576777888 89 AMst PEL TES SPE esca autem nos ] om. $\mathrm{AMst}^{\mathrm{AD}}$, cibus nos TES $\mathrm{S}^{\mathrm{ed}}$, esca TES $\mathrm{S}^{\mathrm{var}}$ non commendat deo 5488 neque ] nec TES ${ }^{\text {var, }}$, quae TES ${ }^{\text {var }}$; enim $5^{1} 5^{8} 61757^{6} 77788889$ AMst PEL $^{\text {B }}$ SPE var

si non $\mathrm{AMst}^{\mathrm{AD}}$ ] si $58617576777889 \mathrm{AMst}^{\mathrm{ed}} \mathrm{PEL}^{\mathrm{B}} \mathrm{TES}$

manducauerimus $\mathrm{AMst}^{\mathrm{AD}}$ ] ederimus $\mathrm{AMst}^{\mathrm{ed}}$, edimus $\mathrm{AMst}^{\mathrm{var}}$, manducabimus TES ${ }^{\text {var }}$

deficiemus ] abundabimus 617677 AMst $^{e d}$ PEL $^{\mathrm{B}}$ TES ${ }^{\text {ed }}$, abundauimus 7578 AMst $^{\text {var }}$ PEL $^{\text {var }}$ TES $^{\text {var }}$, abundamus 89 TES $^{\text {var }}$, deficiamus AMst ${ }^{\mathrm{AD}}$ neque ] quae TE $S^{\text {var }}$ + enim $75^{*}$ si ] + non 617576777889 AMst $^{\text {ed }} \mathrm{PEL}^{\mathrm{B}}$ TES manducauerimus $\mathrm{AMst}^{\mathrm{AD}} \mathrm{TES}^{\mathrm{var}}$ ] ederimus $\mathrm{AMst}^{\mathrm{ed}} \mathrm{TES}^{\mathrm{ed}}$, manducauimus TES var

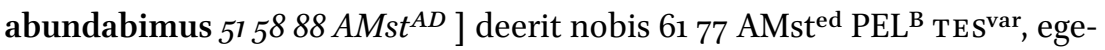
mus 757689 , deficiemus $78 \mathrm{PEL}^{\text {var }}$, abundauimus PEL ${ }^{\mathrm{var}}$, egebimus TES ${ }^{\text {ed }}$

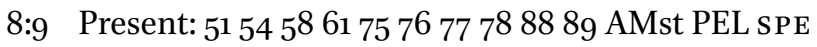

uidete autem ] uidete tamen $\mathrm{AMst}^{\mathrm{ed}}$, uidete ergo $\mathrm{AMst}^{\mathrm{AD}}$ ne forte

haec licentia uestra $51545^{8} \mathrm{AMst}^{\mathrm{D}}$ ] licentia uestra haec 75767789 , licentia uestra $\mathrm{AMst}^{\mathrm{ed}}$, hac licentia uestra $\mathrm{SPE}^{\mathrm{var}}$

offendiculum fiat $\mathrm{AMst}^{\mathrm{AD}}$ ] offensio fiat $\mathrm{AMst}^{\mathrm{ed}}$

infirmibus $\mathrm{PEL}^{\mathrm{var}} \mathrm{SPE}^{\mathrm{var}}$ ] infirmis $51545^{8} 61777888 \mathrm{AMst}^{\mathrm{PEL}} \mathrm{AB}^{\mathrm{AB}} \mathrm{SP}^{\mathrm{ed}}$

8:10 Present: 515458617576777888 89 AMst PEL SPE

si enim quis uiderit ] nam si quis uiderit $757689 \mathrm{AMst}^{\mathrm{AD}}$

eum qui habet ] te qui habet $7576\left(\mathrm{AMst}^{\mathrm{AD}}\right)$, habentem 7778

scientiam 54 ] conscientiam 58 , scientia et $75^{*}$

in idolio recumbentem $5^{1} 5^{8}$ ] in idolo recumbentem 54617588 AMstrar $^{\text {var }}$ $\mathrm{PEL}^{\mathrm{B}} \mathrm{SPE}^{\mathrm{var}}$, recumbentem in idolio SPEvar

nonne conscientia eius 58 ] nonne scientia eius $54^{*} 61^{*}$

cum sit infirma ] infirmantis uel cum sit infirma uel infirmus 77 aedificabitur 51545876 ] aedificatur $77 \mathrm{SPE}^{\mathrm{var}}$; + idolis 61 ad manducandum ] ad edendum $\mathrm{PEL}^{\mathrm{B}}$ idolothyta 51547888 ] idolatica $58 P E L^{B}$, immolatum 61, immolata 757689 $\mathrm{AMst}^{\mathrm{AD}}$, idolothyta uel immolata 77 , de simulacris AMst ${ }^{\mathrm{ed}}$ 
8:11 Present: 51545861757677788889 AMst PEL SPE

et peribit ] et perit $757689 \mathrm{AMst}^{\mathrm{var}}$

infirmus $\mathrm{AMst} \mathrm{t}^{\mathrm{AD}}$ ] qui infirmus est $6177 \mathrm{AMst}^{\mathrm{ed}} \mathrm{PEL}^{\mathrm{B}}$, quia infirmus est AMst ${ }^{\text {var }}$

in tua scientia $\left.5^{C}\right]$ in tua conscientia $5^{1} 54\left\langle 5^{*}\right\rangle 6188 \mathrm{AMst}^{\mathrm{var}} \mathrm{PEL}{ }^{\mathrm{var}} \mathrm{SPE}^{\mathrm{var}}$ frater propter quem ] frater pro quo $61 \mathrm{AMst}^{\mathrm{ed}}$, om. $\mathrm{AMst}^{\mathrm{AD}}$

christus mortuus est ] mortuus est christus 61, christus est mortuus AMst ${ }^{\mathrm{R}}$, om. AMst ${ }^{\mathrm{AD}}$

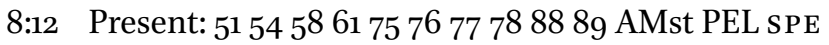

sic autem ] si autem $5475^{*} \mathrm{AMst}^{\mathrm{var}}$, sit 58

peccantes ] peccatores $S P E^{\text {var }}$

in ] inter 61

fratres ] fratribus 58

et percutientes $5888 \mathrm{AMst}^{\mathrm{var}}$ ] percutientes $7576777889 \mathrm{AMst}^{\mathrm{ed}} \mathrm{PEL}^{\mathrm{B}}$

conscientiam eorum $545^{8}$ ] conscientiam ipsorum $6189 \mathrm{AMst}^{\mathrm{AD}} \mathrm{PEL}^{\mathrm{B}}$, ipsorum conscientiam 7576 , eorum conscientiam $77\left(78^{*}\right)^{\mathrm{C}}$, conscientiam illorum AMst ${ }^{\text {ed }}$

infirmam in christo ] infirmam in christum $5^{1} 78 \mathrm{AMst}^{\mathrm{D}} \mathrm{SPE}$

peccatis ] peccantes $88 *$

8:13 Present: 51545861757677788889 AMst PEL SPE

quapropter $\langle 61\rangle$ ] propter quod 757689 AMst, propter quod uel quapropter

77

si esca $7677 \mathrm{PEL}^{\text {var }}$ ] si $88^{*}$ AMst ${ }^{\text {var }} \mathrm{PEL}^{\text {var }}$

scandalizat fratrem 5861 ] scandalizat $\mathrm{PEL}^{\mathrm{B}}$

meum AMst ${ }^{\mathrm{AR}}$ ] om. $75^{*} 7778 \mathrm{AMst}^{\mathrm{ed}} \mathrm{PEL}^{\mathrm{B}}$, nostrum PEL var

non manducabo $\mathrm{AMst}^{\mathrm{AD}}$ ] non edam $\mathrm{AMst}^{\mathrm{ed}}$

carnem ] carnes 58 , om. $\mathrm{AMst}^{\mathrm{D} *}$

in aeternum 5154587688

ne fratrem ] ut non fratrem $5^{8} 7576777889 \mathrm{AMst}^{\mathrm{AD}}$

meum AMst ${ }^{\mathrm{AD}} \mathrm{PEL}^{\mathrm{var}}$ ] om. $7576777889 \mathrm{AMst}^{\mathrm{ed}} \mathrm{PEL}^{\mathrm{AB}}$, eum 88*

scandalizem 61 ] scandalizarem AMst ${ }^{\mathrm{var}}$

9:1 Present: 515458617576777888 89 AMst PEL CAr

non ] nonne $77^{\mathrm{C}}$

sum liber ] sum apostolus 617576777889 AMst PEL $^{\mathrm{B}}$

non ] nonne 77

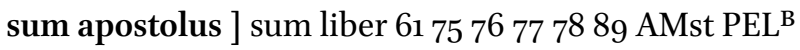

nonne ] non 7576 
iesum dominum nostrum uidi ] christum iesum dominum nostrum uidi $5^{1}$ $5^{8} 77788889 \mathrm{AMst}^{\mathrm{AD}} \mathrm{PEL}^{\mathrm{B}}$, dominum iesum christum uidi 54 , iesum christum dominum nostrum uidi $617576 \mathrm{CAr}$

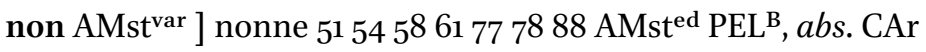
opus meum uos estis in domino ] abs. CAr

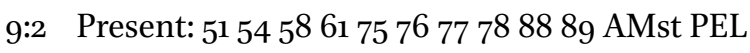
si aliis ] et si aliis $5154586188 \mathrm{AMst}^{\mathrm{R}} \mathrm{PEL}^{\mathrm{B}}$

non sum apostolus ] non solum apostolus 58 , apostolus non sum 89 sed tamen uobis ] si tamen uobis 51 , uobis tamen 61, uerumtamen uobis $\mathrm{AMst}^{\mathrm{ed}}$, uobis $\mathrm{AMst}^{\mathrm{AD}}{ }^{\mathrm{A}}$, tamen uobis $\mathrm{PEL}^{\mathrm{A}}$, at tamen uobis $\mathrm{PEL}^{\mathrm{B}}$, sed tamen uobiscum PEL var

sum nam signaculum

apostolatus mei 61 ] mei apostolatus 7778

uos estis in domino ] uos estis $7576 \mathrm{AMst}^{\mathrm{var}}$

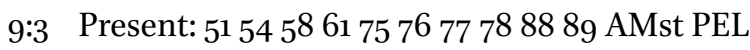

mea defensio 61$]+$ uel excusatio 77

apud eos qui $515878 \mathrm{AMst}^{\mathrm{R}}$ ] ad eos qui $75768889 \mathrm{AMst}^{\mathrm{ed}} \mathrm{PEL}^{\mathrm{var}}$

me interrogant $\left(88^{*}\right)$ ] interrogant me $61 \mathrm{PEL}^{\mathrm{var}}$, me iudicant $7778 \mathrm{AMst}^{\mathrm{AD}}$, me diiudicant interrogant 89

haec est 51545876

9:4 Present: $545^{8} 61757677^{\dagger} 7888$ 89 AMst PEL SPE

numquid non habemus potestatem 54 ] numquid potestatem non habemus 61

\section{manducandi et bibendi}

9:5 Present: 51545861757677788889 AMst PEL sPe

\pm aut $\mathrm{AMst}^{\mathrm{AD}}$

numquid non habemus potestatem 5477 (88)

sororem mulierem $\left(75^{*}\right) \mathrm{AMst}^{\mathrm{AD}}$ ] mulierem sororem 58 , mulieres 6177 (78)

89 AMst $^{\text {ed }}$ PEL $^{\mathrm{A}}$, mulierem $88^{\mathrm{C}} \mathrm{AMst}^{\mathrm{var}}$

circumducendi $(89)]$ circumcidendi $58^{\dagger}$

sicut et ] sic et $54^{*}$, et AMst ${ }^{\mathrm{var}}$

ceteri apostoli 61 ] alii apostoli 78

$\dagger \quad 1$ Cor. 9:4. This verse is duplicated in VL 77 .

$\dagger{ }_{1}$ Cor. 9:5. The abbreviation in VL 58 could also be read as circumducendi. 
et fratres ] fratres 61, et frater 88

domini et cephas 54757888

9:6 Present: $5^{1} 545^{8} 617576777888$ 89 AMst PEL sPe

aut ] an $\mathrm{AMst}^{\mathrm{AD}}$

solus ego ] solum ego $\mathrm{AMst}^{\mathrm{A}}$, ego solus $\mathrm{PEL}^{\mathrm{B}}$

et barnabas 61 ] aut barnabas PEL var

non habemus potestatem 54 (89) ] hoc habemus potestatem AMst ${ }^{\text {var }}$

hoc ] haec 51, non 75767789 AMst, om. PEL ${ }^{\mathrm{B}}$

operandi

9:7 Present: $51545^{861} 7576777884888925^{\dagger}$ 271 AMst PEL SPE

quis militat ] fratres quis militat $8425^{1271}$; + sine 61, + in 271

suis stipendiis 271 ] stipendiis suis AMst ${ }^{\text {var }}$

umquam $5^{8}$ ] aliquando $61 \mathrm{PEL}^{\mathrm{B}}$, abs. 84; + aut $\mathrm{AMst}{ }^{\mathrm{AD}}$

quis ] qui $5^{1} 88^{*}$, abs. 84

plantat uineam 61 ] plantauit uineam $5^{1} \mathrm{AMst}^{\mathrm{var}}$, abs. 84, plata[1] uineam $88^{*}$

et fructum ] et fructus $5^{1} \mathrm{AMst}^{\mathrm{D}}$, et de fructum 547589251 , et de fructu $5^{8}$

617688271 AMst $^{\text {ed }}$ SPE, abs. 84

eius non ] abs. 84, non PEL var

edit $\left(76^{*}\right) 25^{1} \mathrm{AMst}^{\mathrm{var}}$ ] edet $548925^{2}{ }^{2} 21 \mathrm{AMst}^{\mathrm{ed}} \mathrm{PEL}^{\mathrm{B}} \mathrm{SPE} \mathrm{E}^{\mathrm{var}}$, edat $61 \mathrm{SPE}^{\mathrm{var}}$, manducabit uel edit 77 , manducat $78, a b s .84$

\pm et bibit 75767778

quis ] abs. 84, qui $89 *$

pascit ] abs. 84, pascet $89 \mathrm{PEL}^{\mathrm{B}}$

gregem et de lacte] gregem ouium et de lacte $61 \mathrm{AMst}^{\mathrm{ed}}\left(\mathrm{PEL}^{\mathrm{B}}\right), a b s .84$

gregis AMst ${ }^{\text {var }}$ ] gregis ipsius 75 76, ipsius 77, eius $78 \mathrm{AMst}^{\mathrm{AD}} \mathrm{PEL}^{\text {var }}, a b s .84$, earum AMsted

non manducat $25^{1} \mathrm{AMst}^{\mathrm{var}}$ ] non percipit $757689 \mathrm{AMst}^{\mathrm{ed}}$, non edit 7778 , abs. 84 , non comedit $25^{2}$

9:8 Present: $51545^{8} 61757677788889251271$ AMst PEL SPE

numquid secundum hominem $5477(78)$

haec dico $545^{8} 76$ ] dico haec 51 , dico $78 \mathrm{AMst}^{\mathrm{var}}$, hoc dico $271 \mathrm{SPE}^{\mathrm{var}}$, haec loquor AMst ${ }^{\text {ed }}$, loquor $\mathrm{PEL}^{\mathrm{B}}$

an et lex ] an si et lex 7778 , numquid et lex $\mathrm{AMst}^{\mathrm{R}}$

$\dagger \quad$ 1 Cor. 9:7-12. These verses appears in two lections in VL 251: differences are noted by superscript numbers. 
haec non 545876 ] non haec ${ }_{51} \mathrm{PEL}^{\mathrm{var}}$, haec $61777^{8} 89$, non $271 \mathrm{AMst}^{\mathrm{AD}}$, eadem $\mathrm{AMst}^{\text {ed }}$, haec eadem $\mathrm{AMst}^{\mathrm{var}}$, eadem non $\mathrm{AMst}^{\mathrm{R}}$, hoc $\mathrm{PEL}^{\mathrm{B}}$, om. PEL ${ }^{\text {var }}$, hoc non $\mathrm{SPE}^{\mathrm{var}}$

dicit ] loquitur $\mathrm{AMst} \mathrm{AD}^{\mathrm{AD}}$

9:9 Present: 51545861757677788889251271 AMst PEL SPE

scriptum est enim in lege mosi ] scriptum est enim in lege moysi $51545^{8}$ $6188251271 \mathrm{PEL}^{\mathrm{A}} \mathrm{SPE}$, scriptum est enim $75767778 \mathrm{PEL}^{\mathrm{B}}$, in lege enim scriptum est $89 \mathrm{AMst}^{\mathrm{ed}}$, in moysi enim lege enim scriptum est $\mathrm{AMst}{ }^{\mathrm{AD}}$ non alligabis $27^{2} 271$ ] non camum mittes 7576 , non alligabitis AMstvar, non infrenabis $\mathrm{AMst}^{\mathrm{AD}}$, non ligabis $\mathrm{AMst}^{\mathrm{R}}$

os 54271 ] om. 7576

boui trituranti ] bouis triturantis $271 \mathrm{AMst}^{\mathrm{R}}$

numquid de bubus $251^{2}$ ] numquid de bobus $54^{C} 5861757677251^{1} \mathrm{AMst}^{\mathrm{var}}$ SPE ${ }^{\text {var }}$

cura est deo ] est cura deo $5^{8}$

9:10 Present: $51545^{8} 61757677788889251271$ AMst PEL SPE an ] aut potius AMsted, an potius AMstrar

propter nos ] propter uos $\mathrm{PEL}^{\mathrm{B}}$

utique dicit $\mathrm{AMst} \mathrm{AD}^{\mathrm{AD}}$ ] haec utique dicit 58 , haec dicit utique 61, haec dicit 89, hoc dicit 271, dicit AMsted, utique haec dicit PEL ${ }^{\mathrm{B}}$, hoc utique dicit SP $\mathrm{E}^{\mathrm{var}}$; + propter nos 61

nam propter nos 88 ] pro nos utique 75 , pro nobis utique 76 , propter nos enim $777889 \mathrm{AMst}^{\text {ed }}$, nam propter nos utique $271 \mathrm{SPE}^{\mathrm{var}}$, propter nos utique $\mathrm{AMst}^{\mathrm{AD}}$, nam propter uos $\mathrm{PEL}^{\mathrm{B}}$

scripta sunt $25^{2} 271$ ] scriptum est 6175767778 89 AMst; + haec 51

quoniam ] quoniam ipse 61 , quia $7576 \mathrm{AMst}^{\mathrm{AD}}$, qua 89 , quoniam qui 271 AMsted

debet in spe qui arat arare $\left.S P E^{\text {var }}\right]$ in spe qui arat $61(89)$, in spe qui arat debet arare $757678 \mathrm{PEL}^{\mathrm{B}}$, debet qui in spe debet qui arat debet arare $77,^{\dagger}$ in spe arat $\mathrm{AMst}^{\mathrm{ed}}$, debet in spe arare qui arat $\mathrm{AMst}^{\mathrm{AD}} \mathrm{SPE}^{\mathrm{ed}}$

et $\mathrm{AMst}^{\mathrm{var}}$ ] om. $89 \mathrm{AMst}^{\text {ed }}$

qui triturat $271 \mathrm{AMst}^{\mathrm{AD}}$ ] triturat debet $61 \mathrm{AMst}^{\mathrm{var}}$, triturans 77 , debet triturans 89 , triturans debet $\mathrm{AMst}^{\mathrm{ed}}$, qui triturat debet $\mathrm{PEL}^{\mathrm{B}}$

in spe fructus percipiendi (271) ] spei suae fructum percipere 6175767789 $\mathrm{AMst}^{\mathrm{ed}} \mathrm{PEL}^{\mathrm{B}}$, sub spe percipiendi triturat $\mathrm{AMst}{ }^{\mathrm{AD}}$

$\dagger \quad$ 1Cor. 9:10. The Latin translator in VL 77 has confused the segmentation of the Greek. 
9:11 Present: $51545^{8} 61757677788889251271$ AMst PEL sPE si PEL ${ }^{\text {var }}$ ] si ergo 61, om. $89 \mathrm{PEL}^{\mathrm{B}}$

nos uobis spiritalia $5^{1} 5477$

seminauimus ] seminabimus 271 ; + non $75\left\langle 89^{*}\right\rangle \mathrm{AMst}^{\mathrm{AD}} \mathrm{PEL}^{\mathrm{B}}$

magnum est ] magnum 757677

si ] ut 271

nos AMst ${ }^{\mathrm{var}}$ ] om. $7888 \mathrm{AMst}^{\mathrm{ed}} \mathrm{PEL}^{\mathrm{var}}$ SPE

carnalia uestra metamus $P E L^{v a r} S P E^{\text {var }}$ ] uestra carnalia metamus 546175 7677788889271 AMst PEL ${ }^{A B} S_{P E}{ }^{\text {ed }}$

9:12 Present: $5^{1} 545^{8} 61757677788889251271$ AMst PEL SPE si alii

potestatis uestrae $\left.515458 \mathrm{PEL}^{\mathrm{var}}\right]$ uestrorum potestatem 7576 , uestrae potestatis 7778 , uestram potestatem 89 , potestati uestrae $271 \mathrm{PEL}^{\mathrm{B}}$, potestate uestra $\mathrm{AMst}^{\mathrm{ed}}$, uestri potestatem $\mathrm{AMst}^{\mathrm{AD}}$

participes sunt ] habent $757689 \mathrm{AMst}^{\mathrm{AD}}$, utuntur AMst ${ }^{\mathrm{ed}}$; + quare $51545^{8}$ $617888^{*} \mathrm{SPE}^{\mathrm{var}}$

non potius nos ] nos non potius 61, non magis nos $7576 \mathrm{AMst}^{\mathrm{var}}$, non magis uel potius nos 77 , nonne potius nos $88^{\mathrm{C}}$, nonne magis nos $\mathrm{AMst}^{\mathrm{ed}}$ sed non usi sumus $61 \mathrm{AMst}^{\mathrm{D}}$ ] sed nos usui sumus 271, sed non sumus usi $\mathrm{AMst}^{\mathrm{ed}}$

hac potestate 88 ] de hac potestate 58 , potestate hac 7778 , hac potestatem $251^{1}$, hanc potestatem $\mathrm{AMst}^{\mathrm{AD} *} \mathrm{PEL}^{\text {var }}$

sed omnia ] om. 271, omnia uero $\mathrm{AMst}^{\mathrm{R}}$

sustinemus ] sufferimus $7576777889 \mathrm{AMst}^{\mathrm{AD}}$, om. 271, toleramus AMst ${ }^{\text {ed }}$ ne quod ] ut non 77 (78), nec quod $\mathrm{PEL}^{\mathrm{B}}$

offendiculum $\mathrm{AMst}^{\mathrm{AD}}$ ] offendemus uel offendiculum 77 , impedimentum AMsted

demus euangelio christi 545861 ] demus euangelio dei 78

9:13 Present: $5^{1} 545^{8} 61757677788889251271$ AMst PEL sPE

nescitis quoniam ] nescitis quia $\mathrm{AMst}^{\mathrm{AD}}$

qui ] hii qui 58 , om. $271^{*} \mathrm{PEL}^{\mathrm{B}}$ *

in sacrario $\mathrm{AMst}{ }^{\mathrm{AD}}$ ] in sacrariis $6175767789 \mathrm{AMst}^{\mathrm{ed}} \mathrm{PEL}^{\mathrm{B}}$

operantur ] om. 271

quae $51545^{8} 77$ ] om. 757689271 AMst $^{\text {ed }}$, ea quae AMst ${ }^{\mathrm{AD}}$

de sacrario $\left(54^{*}\right)$ ] in sacrario $61^{*}$, om. 78 271, de sacrariis $\mathrm{PEL}^{\mathrm{B}}$

sunt $\mathrm{AMst}^{\mathrm{AD}}$ ] om. $757689271 \mathrm{AMst}^{\mathrm{ed}}$, desunt 78

edunt 61 AMst $^{\mathrm{AD}}$ ] edent 89 , uiuunt $\mathrm{AMst}^{\mathrm{ed}}$

qui ] et qui $5^{1} 545^{8} 271 \mathrm{AMst} P \mathrm{LL}^{\mathrm{var}} \mathrm{SPE}^{\mathrm{var}}$ 
altario ] altari $5188^{*} \mathrm{AMst}^{\mathrm{var}}$, de altario $\mathrm{PEL}^{\mathrm{var}}$

deseruiunt 271 ] seruiunt AMst ${ }^{\mathrm{var}} \mathrm{PEL}^{\mathrm{var}}$; + uel deseruientes 77

cum ] de 6177271 AMst $^{\text {var }} \mathrm{PEL}^{\mathrm{B}}$, om. AMst ${ }^{\mathrm{ed}}$

altario ] altari $5^{1} 88^{*} \mathrm{AMst}^{\mathrm{var}}$

participantur ] compartiuntur uel participant 77 , participant $89 \mathrm{AMst}^{\mathrm{A}}$

PEL var

9:14 Present: 51545861757677788889251271 AMst PEL SPE

ita et ] ita $88^{*} \mathrm{AMst}^{\mathrm{AD}} \mathrm{PEL}^{\mathrm{B}} \mathrm{SPE}^{\mathrm{var}}$; + nos 271

dominus $\left.\mathrm{AMst}^{\mathrm{AD}}\right]+$ iesus AMst ${ }^{\mathrm{ed}}$

ordinauit 54271 ] disposuit $\left(75^{*}\right)^{\mathrm{C}} 7689 \mathrm{AMst}^{\mathrm{AD}}$, disposuit uel ordinauit 77 , or $[2-4]$ dinauit $88^{*}$

his qui $58 \mathrm{AMst}^{\mathrm{AD}}$ ] eos qui AMsted, eis qui $\mathrm{AMst}^{\mathrm{var}}$

euangelium 545861 ] euangelio 271

adnuntiant $51545^{8} 7788$ ] denuntiant AMst' ${ }^{\text {var }}$; ut $77^{*} 89$

de euangelio uiuere 5861271 ] de euangelio uiuant $757689 \mathrm{AMst}^{\mathrm{AD}}$

9:15 Present: 51545861757677788889251 271 AMst PEL SPE

ego autem ] ego his $\mathrm{AMst}{ }^{\mathrm{AD}}$

nullo horum ] nihil horum 61 PEL ed, nullo eorum $757^{6}$, non 77 78, nihilorum $271 \mathrm{PEL}^{\mathrm{B}}$

usus sum 61 ] iussus sum $75^{*}$; + nihil uel nullum horum 77 , + ullo horum 78 non scripsi autem haec 5154587789251 ] non autem scripsi haec 7576271 $\mathrm{AMst}^{\mathrm{ed}}$, non his haec scripsi AMst ${ }^{\mathrm{AD}}$

ut ita fiant in me $\mathrm{AMst}^{\mathrm{AD}}$ ] ut ita in me fiant $5^{1} 5^{8} \mathrm{AMst}^{\mathrm{var}}$, ut ita fiat in me (75) $76 \mathrm{AMst}^{\mathrm{ed}}$

bonum est enim mihi $5458271 \mathrm{AMst}^{\mathrm{var}}$ ] bonum est mihi ${ }_{51} 61 \mathrm{AMst}^{\mathrm{ed}} \mathrm{PEL}^{\text {var }}$, bonum enim mihi est 7576777889

magis mori quam $\mathrm{AMst}^{\mathrm{var}}$ ] magis mori $89 \mathrm{AMst}^{\mathrm{ed}} \mathrm{PEL}^{\mathrm{B}}$

ut AMstrar ${ }^{\text {var }}$ om. $75767788^{*} 89$ AMsted $^{\text {ed }}$ PL $^{\mathrm{B}}$

gloriam meam ] gloria mea 271, gloriam autem meam $\mathrm{PEL}^{\mathrm{B}}$

quis AMst ${ }^{\mathrm{var}}$ ] nemo $757689 \mathrm{AMst}^{\mathrm{ed}} \mathrm{PEL}^{\mathrm{B}}$

euacuet $A M s t^{\text {var }}$ ] euacuat 61 , exinaniet 7576 , euacuat uel exinaniat 77 , euacuauit 89, euacuabit AMst $^{\text {ed }}$ PEL $^{\mathrm{B}}$

9:16 Present: 51545861757677788889 271 AMst PEL CAr SPE

nam si ] si enim $7576777889 \mathrm{AMst}^{\mathrm{AD}}$, nam 271 , nam et si SPE $\mathrm{E}^{\mathrm{var}}$

euangelizauero $545^{8} 61$ (78) $\left.271 \mathrm{AMst}^{\mathrm{var}}\right]$ euangelizem $75767789 \mathrm{AMst}^{\mathrm{ed}}$

$\mathrm{PEL}^{\mathrm{B}} \mathrm{CAr}$, scandalizauero PEL ${ }^{\text {var }}$

non est mihi 515458271 ] non mihi PEL var 
gloria AMstvar ] gratia $75767778 \mathrm{AMst}^{\mathrm{ed}}$, gloriatio $\mathrm{AMst}^{\mathrm{R}}$ necessitas enim ] necessitas AMstrar, abs. CAr mihi incumbit uae enim 515458617677 (78) 271 ] abs. CAr mihi est $51545^{8} 271$ ] mihi 61 , mihi erit $78 \mathrm{AMst}^{\mathrm{AD}}$, erit mihi AMsted, est mihi $\mathrm{AMst}^{\mathrm{var}}$, om. PEL ${ }^{\mathrm{var}}$, abs. CAr; + uel erit 77 si non ] abs. CAr euangelizauero $545861271 \mathrm{AMst}^{\mathrm{var}}$ ] euangelizem 757689 , euangelizem uel non euangelizauero 77, praedicauero euangelium AMst ${ }^{\mathrm{ed}}$, abs. CAr

9:17 Present: 51545861757677788889 271 AMst PEL sPE si enim ] nam si 757689 AMst $^{\text {ed }}$, nam et si AMstrar uolens ] + uel cupiens 77 hoc ago mercedem habeo si autem inuitus dispensatio 58271 mihi credita est 515458271 ] credita est mihi 77 ; + per fidem quae est in christo iesu domino nostro amen $271^{\dagger}$

9:18 Present: 51545861757677788889 AMst PEL SPE quae 515458617688 ] qua $77^{*}$, quid $77^{C}$

est ergo $\left(88^{*}\right)$ ] igitur erit 7576 , ergo erit $777^{8} \mathrm{AMst}^{\text {ed }}$, igitur est $89 \mathrm{AMst}^{\mathrm{AD}}$, est enim PELA, ergo est PEL var

merces mea PEL ${ }^{\text {var }}$ ] mea merces $61 \mathrm{PEL}^{\mathrm{B}}$, mihi merces $75767778 \mathrm{AMst}^{\mathrm{AD}}$, mea mercis $\mathrm{AMst}^{\mathrm{ed}}$, mercis mea AMst ${ }^{\text {var }} \mathrm{PEL}^{\mathrm{A}}$ ut ] si 89 euangelium praedicans $5154586188 \mathrm{AMst}^{\mathrm{var}}$ ] euangelizans 75767778 $\mathrm{AMst}^{\mathrm{AD}}$, euangelium adnuntians $89 \mathrm{AMst}^{\mathrm{ed}}$ sine sumptu ponam $\mathrm{AMst}^{\mathrm{R}}$ ] sine sumptu efficiam (75) (76) $89 \mathrm{AMst}^{\mathrm{ed}}$ $\mathrm{PEL}^{\mathrm{B}}$

euangelium 545861$]+$ christi $587778 \mathrm{SPE}$

ut non abutar ] ut abutar $S \mathrm{PE}^{\mathrm{var}}$

potestate mea ] potestatem meam $89 \mathrm{PEL}^{\mathrm{var}}$

in euangelio $545861\left(88^{*}\right)$

9:19 Present: 51545861757677788889 AMst PEL SPE nam cum

liber essem 54 ] libera sim $75^{*}$, liber $\operatorname{sim} 75^{\mathrm{C}} 7689$ AMsted $S P E^{\text {var }}$, sim liber AMst $^{\text {var }}$ ex ] ab $7576777889 \mathrm{AMst}^{\mathrm{AD}}$

$\dagger 1$ Cor. 9:17. This is a standard conclusion to a lection in VL 271. 
omnibus ] hominibus $\mathrm{SPE}^{\mathrm{var}}$

omnium ] in omnium $75^{*}$, in omnibus $75^{\mathrm{C}} 76$, omnibus 77 , hominibus 89 , om. $\mathrm{AMst}^{\mathrm{AD}}$

me seruum ] seruum me 6189

feci ] fecit $P E L^{B}$

ut plures $\left(88^{*}\right)$ ] ut omnes $\mathrm{AMst} \mathrm{AD}^{\mathrm{AD}}$

lucri facerem AMstrar $]$ lucri faciam $6175\langle 76\rangle 89$ AMsted, lucrificarem $_{77}$ SPEvar

\pm ad fidem christi 58

9:20 Present: $51545^{8} 61757677788889$ AMst PEL SPE

et factus sum ] fui 7576 , et fui 89 ; + uel fui 77

iudaeis tamquam iudaeus $515458617888 \mathrm{AMst}^{\mathrm{var}}$ ] iudaeis quasi iudaeus 75 $\left(76^{*}\right)^{c} 89 \mathrm{AMst}^{\mathrm{ed}}$, quasi iudaeus iudaeis 77

ut iudaeos lucrarer $51545888 \mathrm{AMst}^{\mathrm{AD}}$ ] ut iudaeos lucrificarer 61 , ut iudaeos lucri facerem 75767889 AMst $^{\text {ed }}$, ut iudaeos lucrificarem 77 , ut iudaeis lucrum facerem AMst ${ }^{\mathrm{var}}$, ut lucrarer iudaeos $\mathrm{SPE}^{\mathrm{var}}$

his 58 ] om. 78 , iis AMst ${ }^{\mathrm{R}}$

qui sub lege sunt ] qui sub lege 7778

quasi ] quasi uel tamquam 77

sub lege essem cum 54 ] sub lege cum 757677

ipse non essem sub lege ] ipse sub lege non essem 54 , ipse sub lege non sum

61 , non essem ipse sub lege 7778 , ipse non sim sub lege $89 \mathrm{AMst}^{\mathrm{ed}} \mathrm{PEL}^{\mathrm{B}}$, ipse non sub lege essem AMst ${ }^{\mathrm{AD}}$; + sed sub gratia SPE ${ }^{\text {ed }}$

ut eos qui ] ut qui 7576 , ut his qui $89^{*}$

sub lege erant $\left(89^{*}\right) \mathrm{AMst}^{\mathrm{AD}}$ ] sub lege sunt $61 \mathrm{AMst}^{\mathrm{ed}} \mathrm{PEL}^{\mathrm{B}}$, sunt sub lege 75 76

lucri facerem ] lucri faciam $61 \mathrm{PEL}^{\mathrm{B}}$, lucrarem $\left(75^{*}\right)^{\mathrm{C}} 76$, lucrarer $777^{8}$

9:21 Present: 51545861757677788889 AMst PEL SPE

his 58 ] iis $\mathrm{AMst}^{\mathrm{R}}$, om. PEL $^{\mathrm{B}}$

qui sine lege erant ] qui sine lege sunt 61 (75) 767778 89 AMst $\mathrm{PEL}^{\mathrm{B}}$

tamquam $77 \mathrm{AMst}^{\mathrm{AD}}$ ] sicut 75 76, quasi $\mathrm{AMst}^{\mathrm{ed}}$

sine lege $(51)\left(75^{*}\right)$

essem 54 ] om. $757_{6} 68$, sim PEL $^{\text {B }}$

\pm ut lucri facerem eos qui sine lege sunt PEL ${ }^{\text {ed }}$, ut lucri facerem eos qui sine lege erant $\mathrm{PEL}^{\mathrm{A}}$

cum sine lege dei non essem 54 ] cum sine lege dei non sim $617576 \mathrm{PEL}^{\mathrm{B}}$, cum non sim sine lege dei 77 , cum non essem sine lege dei 78 , cum sine lege non essem dei 89 AMst 
sed in lege ] sed sub lege PEL ${ }^{\mathrm{A}}$

essem christi ] sim christi 61757689 PEL $^{B}$

ut ] om. PELA

lucri facerem ] lucrificarem 61 SPE ${ }^{\mathrm{var}}$, om. 757689 AMst PELA, lucrarer 77 78, lucri faciam $\mathrm{PEL}^{\mathrm{B}}$

eos qui sine lege ] eos qui sub lege AMstvar, om. PEL ${ }^{\mathrm{A}}$

erant $\mathrm{AMst}^{\mathrm{AD}}$ ] sunt $61777^{8} \mathrm{AMst}^{\text {ed }} \mathrm{PEL}^{\mathrm{B}}$, om. PEL $^{\mathrm{A}}$

\pm lucri facerem $757689 \mathrm{AMst}^{\mathrm{ed}}$, lucrificarem $\mathrm{AMst}^{\mathrm{A}}$

9:22 Present: 51545861757677788889 AMst PEL SPE

factus sum ] et factus sum 61, fui $757689 \mathrm{AMst}^{\mathrm{AD}}$, fui autem et 77 , factus sum autem et 78 , factus $88^{*} \mathrm{SPE}^{\mathrm{var}}$

infirmis ] infirmus $6188^{*} \mathrm{AMst}^{\mathrm{AD}}$, infirmibus $757689 ;+$ tamquam 7778 $\mathrm{AMst}^{\mathrm{AD}}$

infirmus ut infirmos ] infirmis ut infirmos 61

lucri facerem ] lucri faciam $61 \mathrm{AMst}^{\mathrm{var}} \mathrm{PEL}^{\mathrm{B}}$, lucrificarem 7788 , lucrarer $\mathrm{AMst}^{\mathrm{AD}}$

omnibus omnia factus sum ] omnibus factus sum omnia 75767778 AMst, omnia omnibus factus sum $\mathrm{PEL}^{\mathrm{B}}$

ut omnes ] et omnes $75^{*}$

facerem saluos $\mathrm{SPE}^{\mathrm{var}}$ ] saluos facerem $5^{8} 777^{8} \mathrm{AMst}$, lucri faciam 61, saluos faciam $7576 \mathrm{PEL}^{\mathrm{B}}$, lucri facerem $89 \mathrm{SPE}^{\text {ed }}$, faciam saluos $\mathrm{PEL}^{\mathrm{var}}$

9:23 Present: 515458617576777888 89 AMst PEL SPE

omnia autem facio ] omnia facio $54 \mathrm{PEL}^{\mathrm{var}}$

propter euangelium 545861 ] propter $89 ;+$ christi $88^{\mathrm{C}}$

ut particeps $546 \mathrm{r}$ ] ut participes $5^{8} \mathrm{AMst}^{\mathrm{var}}$, ut socius $757689 \mathrm{AMst}^{\mathrm{AD}}$, ut uel conscius consocius uel particeps 77

eius efficiar 54 ] efficiar $61^{*}$

9:24 Present: 5154586175767778848889 AMst PEL tes FOR SPE

nescitis ] fratres scitis 84

quod AMstvar ${ }^{\text {ver }}{ }^{\text {var }}$ ] quoniam $75767789 \mathrm{AMst}^{\text {ed }} \mathrm{PEL}^{\mathrm{B}} \mathrm{FOR}^{\mathrm{var}} \mathrm{SPE}^{\mathrm{ed}}$, quia $84 \mathrm{AMst}^{\mathrm{AD}}$ TES FOR ${ }^{\mathrm{ed}}$

hii qui $\left.51777^{8} 88^{C} P E L^{\text {var(ed) }}\right]$ qui $757^{6} 8489{\text { AMst } \text { PEL }^{B} \text { TES }}^{\text {ed }}$ FOR, duo TES $^{\mathrm{var}}$, om. TES ${ }^{\mathrm{var}}$

in stadio ] + suo 84

currunt ] + uel currentes $77, a b s .84$

omnes quidem currunt ] abs. 84, omnes currunt PEL var

sed unus $\mathrm{AMst}^{\mathrm{AD}} \mathrm{FOR}^{\mathrm{var}}$ ] unus autem $75767789 \mathrm{AMst}^{\mathrm{ed}} \mathrm{PEL}^{\mathrm{var}} \mathrm{FOR}^{\mathrm{var}}$, abs.

84, unus tamen $\mathrm{TES}^{\text {ed }} \mathrm{FOR}^{\mathrm{ed}}$, unus quidem TES ${ }^{\mathrm{var}}$ 
accipit brauium $7577\left(78^{*}\right)$ ] bradium accipit 61, abs. 84, accepit brauium 89 $P E L^{A}$, accipit palmam AMsted $\mathrm{TES}^{\mathrm{ed}}$ FOR, accepit palmam $\mathrm{AMst}^{\mathrm{var}}$, accipit coronam TES ${ }^{\text {var }}$, accipit bradium TES ${ }^{\mathrm{var}}$

\pm ego autem dico uobis $617778 \mathrm{PEL}^{\mathrm{B}}$

sic currite ut ] abs. 84

conprehendatis 51587788 ] omnes conprehendatis $6189 \mathrm{PEL}^{\mathrm{B}} \mathrm{TES}^{\mathrm{var}}$, abs.

84 , occupetis TES ${ }^{\text {ed }} \mathrm{FOR}^{\text {ed }}$, adprehendatis omnes FOR ${ }^{\mathrm{var}}$

9:25 Present: 515458617576777888 89 AMst PEL tes FOR SPM SPE

omnis FOR ${ }^{\text {var }}$ ] omnes 6175 , abs. TES FOR ${ }^{\text {ed }}$

autem qui ] enim qui $61 \mathrm{AMst}^{\mathrm{AD}} \mathrm{FOR}^{\mathrm{var}} \mathrm{SPE}^{\mathrm{var}}$, abs. TES FOR ${ }^{\mathrm{ed}}$

in agone contendit $\left.\left(88^{*}\right)\right]$ in agone contendunt 61, agonizat uel in agone contendit 77, agone contendit PEL ${ }^{\mathrm{var}}$, abs. TES FOR ${ }^{\text {ed }}$, agonizatur FOR ${ }^{\mathrm{var}}$ ab omnibus ] abs. TES FOR ${ }^{\text {ed }}$, in omnibus FOR ${ }^{\mathrm{var}}$, om. SPM ${ }^{\mathrm{var}}$; + uel omnia 77

se abstinet (76) $\mathrm{AMst}^{\mathrm{var}} \mathrm{SPM}^{\mathrm{var}}$ ] se abstinent 61 , abstinet $\mathrm{AMst}^{\mathrm{ed}}$, continens est $\mathrm{AMst}^{\mathrm{A}} \mathrm{FOR}^{\mathrm{var}} \mathrm{SPM}^{\mathrm{ed}}$, abstinens est $\mathrm{AMst}^{\mathrm{D}}$, abs. TES FOR ${ }^{\mathrm{ed}}$; + ab omnibus SPM ${ }^{\text {var }}$

et illi quidem ] illi quidem 61757677 , om. PEL ${ }^{\mathrm{A}}$

ut ] om. $54^{*}$ PEL ${ }^{\mathrm{A}}$

corruptibilem coronam 5861 ] incorruptibilem coronam $54^{*}$ TES $^{\text {var }}$, om. PEL ${ }^{\mathrm{A}}$

accipiant ] accipiunt $54^{*}$, accipiạnt $54^{\mathrm{C}}$, om. PEL ${ }^{\mathrm{A}}$

nos FOR $\left.{ }^{\text {var }}\right]$ om. PEL ${ }^{\mathrm{A}}$, uOS TES ${ }^{\mathrm{var}} \mathrm{FOR}^{\text {ed }}$

autem ] om. PEL $^{\mathrm{A}}$, uero TES ${ }^{\mathrm{var}}$, tamen TES $^{\mathrm{var}}$

incorruptam $61 \mathrm{AMst}^{\mathrm{var}}$ ] incorruptibilem $757689 \mathrm{AMst}^{\mathrm{ed}} \mathrm{TES}^{\mathrm{var}} \mathrm{FOR}^{\mathrm{var}}$, incorruptibilem uel incorruptam 77, om. PEL ${ }^{\mathrm{A}}$

9:26 Present: $51545^{8} 61757677788889$ AMst PEL SPM SPE

ego igitur 61 ] ego quidem $757^{6} 77$, ego autem PEL var

sic curro ] si curro $8889^{*}$

non quasi ] ut $61 \mathrm{PEL}^{\mathrm{B}}$

in incertum ] incertum 61757689 , in incerto $\mathrm{SPE}^{\mathrm{var}}$

sic pugno ] sic pu[2-3]o $88^{*}$, sic enitor $\mathrm{AMst}^{\mathrm{R}}$, sic certo $\mathrm{PEL}^{\mathrm{B}}$

non quasi PEL $\left.{ }^{\text {var }}\right]$ non ut $P E L^{\text {ed }}$

aerem PEL ${ }^{\text {var }}$ ] aeram 75 , aera $76 \mathrm{AMst}^{\mathrm{R}} \mathrm{PEL}^{\mathrm{AB}}$; + uel aera 77

uerberans AMst ${ }^{\text {var }}$ SPM $^{\text {var }}$ ] caedens 757689 AMst $^{\text {ed }}$ PEL $^{\text {ed }}$ SPM $^{\text {ed }}$, caedens uel uerberans 77 , credens $\left(\mathrm{PEL}^{\mathrm{B}}\right)$ 
9:27 Present: $51545^{8} 61757677788889$ AMst PEL SPM SPE

sed castigo $\left.\mathrm{SPM}^{\mathrm{var}}\right]$ sed liuidum facio $757^{6} 89^{C} A M s t^{A D} \mathrm{SPM}^{\text {ed }}$, libido facio

89 ; , subiugans $\mathrm{SPM}^{\mathrm{var}}$; + uel liuidum facio 77

corpus meum et ] meum corpus et 77

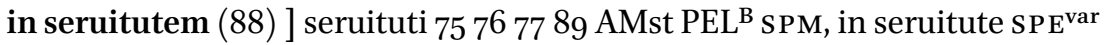

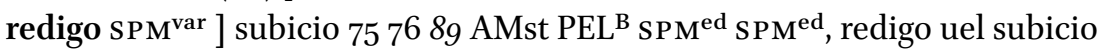

77

ne forte cum AMst ${ }^{\mathrm{AD}}$ ] ne forte $77 \mathrm{AMst}^{\mathrm{ed}} \mathrm{SPM}^{\mathrm{var}}$, ne dum SPM ${ }^{\text {ed }}$

praedicauerim $5154586176(88) \mathrm{AMst}^{\mathrm{AD}}$ ] praedicans $77 \mathrm{AMst}^{\mathrm{ed}} \mathrm{SPM}^{\mathrm{var}}$, praedicant $S \mathrm{PM}^{\mathrm{var}}$

ipse reprobus efficiar $\left(54^{*}\right) 88$ ] ipse reprobus inueniar AMst $\mathrm{SPM}^{\mathrm{var}}$

10:1 Present: 5154586175767778848889 251 AMst PEL CAr TES SPE nolo enim ] nolo autem 78 PEL ${ }^{\text {var }} \mathrm{CAr}$, fratres nolo 84, nolo ergo PEL var uos ] om. 61

ignorare fratres $(78)]$ ignorare $84 \mathrm{TES}^{\mathrm{var}}$, fratres ignorare $\mathrm{AMst}^{\mathrm{var}}$

quoniam ] quod $75768489 \mathrm{AMst}^{\mathrm{AD} *}$, quoniam uel quod 77 , quia $\mathrm{AMst}^{\text {ed }}$

TES

patres nostri omnes sub nube ] patres [abs.] 84

fuerunt ] erant 7576 , abs. 84

et omnes AMst ${ }^{\mathrm{var}}$ ] et omnes per $75767789 \mathrm{AMst}^{\mathrm{ed}}$, abs. 84 CAr TES SPE

mare transierunt ] mare transirent 88 , abs. 84 CAr TES SPE

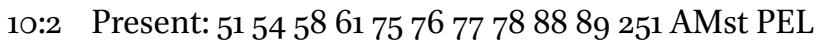

et omnes in mose ] et omnes in moyse $5154587888251 \mathrm{AMst}^{\mathrm{var}} \mathrm{PEL}^{\mathrm{var}}$, et omnes in moysen $6175767789 \mathrm{PEL}^{\mathrm{AB}}$, et omnes in mosen $\mathrm{AMst}^{\mathrm{ed}}$

baptizati sunt in 5461

nube et in mari ] mari et in nube 7778 , nube et in mare $89 \mathrm{PEL}^{\mathrm{var}}$

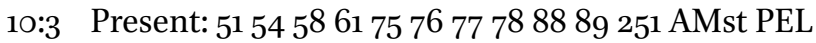

et omnes eandem escam spiritalem ${ }_{51} 61$ (75) (78) 89

manducauerunt $\mathrm{AMst}^{\mathrm{var}}$ ] ederunt $\mathrm{AMst}^{\mathrm{ed}}$

10:4 Present: 51545861757677788889251 AMst PEL

et omnes eundem potum spiritalem biberunt $57\left(54^{*}\right)$

bibebant 251 ] om. 61, bibant $75^{*}$

autem AMst $^{\text {var }}$ ] enim 7576777889 AMsted $^{\text {ed }}$ LLar $^{\text {var }}$

de spiritali ${ }_{51} \mathrm{AMst}^{\mathrm{AD}}$ ] spiritalia 75 , spiritali 76 , de $\mathrm{AMst}^{\mathrm{ed}}$

consequenti $\mathrm{AMst}^{\mathrm{D}}$ ] consequente $5^{1} 5^{8} 88$, sequenti $7576777889 \mathrm{AMst}^{\mathrm{ed}}$, sequente $\mathrm{AMst}^{\mathrm{R}}$ 
eos petra $A M s t^{R}$ ] se petra $757^{6}$, petra 777889 , spiritali petra $A M s t^{\text {ed }}$ petra autem ] petra enim $\mathrm{AMst}^{\mathrm{var}}$, petra uero $\mathrm{AMst}^{\mathrm{AD}}$ erat christus

10:5 Present: 51545861757677788889251 AMst PEL SPE sed non in ] sed non 7576 PEL $^{\text {var }}$, sed in AMstvar

pluribus eorum ] omnibus illis 757689 , pluribus illorum AMst ${ }^{\mathrm{A}}$, plures eorum SPEvar

beneplacitum est ] uoluntas fuit 757689 , beneplacuit 77 78, bene sensit $\mathrm{AMst}^{\mathrm{ed}}$, beneplacitum fuerit $\mathrm{AMst}^{\mathrm{AD}}$

deo $\left.\mathrm{AMst}^{\mathrm{AD}}\right]$ dei $75\langle 76\rangle 89$, deus $7778 \mathrm{AMst}^{\mathrm{ed}}$, om. AMst ${ }^{\mathrm{var}}$

nam prostrati sunt ] prostrati enim sunt (75) $7689 \mathrm{AMst}^{\mathrm{ed}}$, prostrati sunt enim 7778 , om. $88^{*}$, protracti enim sunt $\mathrm{AMst}^{\mathrm{A} *}$

in deserto 61 ] om. $88^{*}$

10:6 Present: $5^{1} 545^{8} 61757677788889251$ AMst PEL SPE

haec autem 51545876 ] + omnia $88^{*}$

in figura (88) ] in figuram $7576 \mathrm{AMst}^{\mathrm{var}} \mathrm{PEL}^{\mathrm{A}}$

facta sunt nostri ut non ] nostri facta sunt ut non 61 (75) 76777889 AMst simus ] efficiamur 7576 , om. $89 \mathrm{AMst}^{\mathrm{AD}}$, sumus $\mathrm{PEL}^{\mathrm{B}}$; + nos 75767778

concupiscentes $\mathrm{AMst}^{\mathrm{var}}$ ] concupiscamus $89 \mathrm{AMst}^{\mathrm{AD}}$, desiderantes $\mathrm{AMst}^{\mathrm{ed}}$ malorum AMst ${ }^{\text {var }}$ ] malum $75^{*}$, mala 89 AMst $^{\text {ed }}$

sicut et illi concupierunt $\mathrm{AMst}^{\mathrm{var}}$ ] sicut et illi desiderauerunt $\mathrm{AMst}^{\mathrm{ed}}$

10:7 Present: 51545861757677788889251 AMst PEL SPE

neque ] nec $757678 \mathrm{AMst}^{\mathrm{AD}}$, ne uel nec 77

idolorum cultores ] idolatrae $5^{1} 54^{C} 5^{8 P E L^{B}} \mathrm{SPE}$, idolatr[1-2] $54^{*}$, idolatriae $61 \mathrm{PEL}^{\mathrm{var}}$, idolatres uel idolorum cultores 77 , idolatres $7888 \mathrm{SPE}^{\mathrm{var}}$, idola colamus $89 \mathrm{AMst}^{\mathrm{AD}}$, idolatrii 251, simulacris seruientes $\mathrm{AMst}^{\mathrm{ed}}$, idololatriae $P E L^{A}$

efficiamini ] efficiamur $7576 \mathrm{PEL}^{\mathrm{B}}$, effici uel efficiamini 77, om. 89 AMst sicut ] om. 77 78; + et 51

quidam ] om. 7778 , aliqui $\mathrm{PEL}^{\mathrm{B}}$

ex ipsis ] ex illis 757689 AMst $^{\mathrm{AD}}$, om. 77 78, illorum AMst Ad $^{\text {ed }}$

quemadmodum ] sicut $7576777889 \mathrm{AMst}^{\mathrm{ed}}$, sicuti AMst ${ }^{\mathrm{AD}}$, om. AMstrar scriptum est 251 ] + in exodo PELvar

sedit ] om. 77

populus manducare et bibere et 251

surrexerunt ludere ] surrexit ludere $S \mathrm{PE}^{\mathrm{var}}$, resurrexerunt ludere $S P E^{\mathrm{var}}$ 


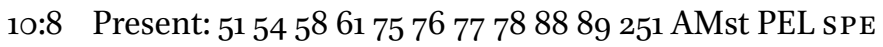
neque fornicemur 251 ] neque fornicemus AMstvar sicut quidam ] sicut 77 ; + fornicati sunt PEL var ex ipsis ] ex illis 7576 89, ipsorum uel ex ipsis 77 , illorum AMst fornicati sunt ] om. PEL var et ceciderunt 61$]+$ in $58,+$ ab SPE $E^{\mathrm{var}}$ una die uiginti tria milia 7589 ] + hominum $6189251 \mathrm{AMst}^{\mathrm{ed}} \mathrm{PEL}^{\mathrm{B}}$

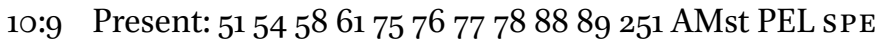
neque temptemus christum ] nec temptemus christum 757689 sicut quidam ] sic quidam $S P E^{\text {var }}$ eorum AMstrar $]$ illorum 89 AMst $^{\mathrm{var}}$, om. AMst ${ }^{\text {ed }}$ temptauerunt et a serpentibus perierunt

10:10 Present: 51545861757677788889251 AMst PEL SPE neque ] ne $\mathrm{AMst}^{\mathrm{A}}$

murmuraueritis 6189251 ] murmuremur 75 76, murmurauerunt 77 sicut quidam eorum $\mathrm{AMst}^{\mathrm{var}}$ ] ex ipsis $61 \mathrm{AMst}^{\mathrm{ed}} \mathrm{PEL}^{\mathrm{B}}$, ex illis $757689 \mathrm{AMst}^{\mathrm{AD}}$ murmurauerunt et perierunt ab exterminatore 6189251

10:11 Present: 51545861757677788889251 AMst PEL SPE haec autem omnia $\left.51545876 \mathrm{AMst}^{\mathrm{var}}\right]$ omnia autem haec 7778 , haec omnia autem $\mathrm{AMst}^{\mathrm{ed}}$, haec autem SPE ${ }^{\mathrm{var}}$ in figura contingebant illis 61 ] in figura facta sunt illis $\mathrm{AMst}^{\mathrm{R}}$ scripta sunt autem ] scripta sunt uero $\mathrm{AMst}^{\mathrm{R}}$, scripta sunt $\mathrm{SPE}^{\mathrm{var}}$ ad ] in 58 correptionem 75 ] corruptionem $54^{*}$, correctionem 58251 AMstrar, commo- $^{\text {va }}$ nitionem $\mathrm{AMst}^{\mathrm{A}}$, admonitionem $\mathrm{AMst}^{\mathrm{D}}$ nostram in quos ] nostram in quo PELvar fines ] fine[2] $54^{*}$, finis $6189 \mathrm{PEL}^{\mathrm{B}}$ saeculorum deuenerunt $51545^{8} 88$ ] saeculorum deuenit 61757677 (78) $\mathrm{PEL}^{\mathrm{B}}$

10:12 Present: 51545861757677788889251 AMst PEL TES SPE itaque $\mathrm{AMst}^{\mathrm{AD}}$ ] propterea et $\mathrm{AMst}{ }^{\mathrm{ed}}$, propterea $\mathrm{AMst}^{\mathrm{var}}$, et TES $\mathrm{S}^{\mathrm{ed}}$, om. TES $^{\mathrm{var}}$ qui se existimat ] qui existimat se $58 \mathrm{PEL}^{\mathrm{A}}$, qui te putant 75 , qui se putat 76 89 AMst $^{\text {TES }}{ }^{\text {ed }}$, qui se putat uel existimat 77 , qui se sperat $\mathrm{TES}^{\mathrm{var}}$ stare uideat ne cadat 
10:13 Present: 51545861757677788889251 AMst PEL TES SPE temptatio uos non $\left.7888^{*}\langle 251\rangle\right]$ temptatio nos non 58 adprehendat $\left.5154^{c} 58767889251 \mathrm{TES}^{\mathrm{var}}\right]$ adprehendit 75 PEL $^{\mathrm{A}} \mathrm{TES}^{\mathrm{var}}$, occupauit TES ${ }^{\text {ed }}$, occupabit TES ${ }^{\text {var }}$, occupet TES ${ }^{\text {var }}$

nisi humana fidelis

autem ] om. 61, itaque SPEvar

deus ] + est $515888\langle 251\rangle$ PEL SPE

qui non TES $\left.{ }^{\mathrm{var}}\right]$ non $\mathrm{TES}^{\mathrm{ed}}$

patietur $545878251 \mathrm{SPE}^{\text {var }}$ ] patitur $6177 \mathrm{TES}^{\mathrm{var}} \mathrm{SPE}^{\text {ed }}$, patiatur AMstvar; + uel non dimittit 77

uos temptari super 251 ] uos temptari supra $5154 \mathrm{AMst}^{\mathrm{var}} \mathrm{PEL}^{\mathrm{var}} \mathrm{SPE}^{\mathrm{var}}$ id quod $\left(54^{*}\right) \mathrm{AMst}^{\mathrm{AD}} \mathrm{TES}^{\mathrm{var}}$ ] quam AMst ${ }^{\mathrm{ed}}$, quod $\mathrm{TES}^{\mathrm{ed}}$, illud quod $\mathrm{TES} \mathrm{S}^{\mathrm{var}}$ potestis $\langle 251\rangle]$ non potestis 75 , non potestis sustinere 7778 , non potestis sufferre 89, ferre potestis TES ${ }^{\text {var }}$

sed faciet $\mathrm{TES}^{\mathrm{var}}$ ] sed faciat $\mathrm{AMst}^{\mathrm{var}} \mathrm{PEL}^{\mathrm{var}} \mathrm{SPE}^{\mathrm{var}}$, et faciet TES $S^{\text {ed }}$ cum temptatione 545878 ] contemptationem 89

etiam ] [2] iam $54^{*}$

prouentum ] prouentu PEL ${ }^{\mathrm{var}} \mathrm{SPE}^{\mathrm{var}}$, euadendi facultatem $\mathrm{TES}^{\mathrm{ed}}$, prouentum facultatem $\mathrm{TES}^{\mathrm{var}}$, iuuandi facultatis $\mathrm{TES}^{\mathrm{var}}$ ut $\left.\left\langle 54^{\mathrm{C}}\right\rangle\right] \mathrm{om} .54^{*}$

possitis 61 ] possetis $\mathrm{PEL}^{\mathrm{A}} \mathrm{SPE}^{\mathrm{var}}$; + uel posse 77

sustinere $\mathrm{AMst}^{\mathrm{AD}} \mathrm{TES}^{\mathrm{var}}$ ] tolerare $\mathrm{AMst}^{\mathrm{ed}} \mathrm{TES}^{\mathrm{ed}}$; + tolerare $\mathrm{AMst}^{\mathrm{var}}$ \pm in christo iesu domino nostro 251

10:14 Present: 51545861757677788889251 AMst PEL CAr SPE

propter quod AMst ${ }^{\mathrm{var}}$ ] propter $77, a b s .251$, quapropter $\mathrm{AMst}^{\mathrm{ed}}$ carissimi 515488

mihi $58 \mathrm{AMst}^{\mathrm{AD}}$ ] om. $515489251 \mathrm{AMst}^{\mathrm{ed}}$, mei 75767778

fugite ab idolorum ${ }_{57}(54) 58(88)$

cultura ] cultu 7576 , seruitute $\mathrm{AMst}^{\mathrm{AD}}$

10:15 Present: 51545861757677788889251 AMst PEL

ut ] sicut $7576777889 \mathrm{AMst}^{\mathrm{AD}}$, quasi AMsted

prudentibus $\mathrm{AMst}^{\mathrm{AD}}$ ] prudentes $757689 \mathrm{AMst}^{\mathrm{ed}} \mathrm{PEL}^{\mathrm{B}}$

loquor uos ] uobis dico 7576 , dico $7778 \mathrm{AMst}^{\mathrm{AD}}$, uos $89 \mathrm{AMst}^{\mathrm{ed}} \mathrm{PEL}^{\mathrm{B}}$

iudicate ] ipsi iudicate $5^{1}\left(54^{*}\right)^{\mathrm{C}} 5^{8} 88$, iudicate uos $75767778 \mathrm{AMst}^{\mathrm{D}}$, iudicate nos $\mathrm{AMst}^{\mathrm{A}}$

quod dico ] quae dico AMstrar 
10:16 Present: 51545861757677788889251 AMst PEL

calicem $88^{\text {alt }}$ ] calix $5^{1} 5^{\mathrm{C}} 5^{8} 61757^{6} 777^{8} 888925^{1} \mathrm{AMst}^{\mathrm{var}} \mathrm{PEL}^{\mathrm{B}}$, cali[2] $54^{*}$

benedictionis cui (54) ] benedictionis quem 6175767778 89 AMst PEL ${ }^{\mathrm{B}}$

benedicimus ] benedicimur AMst ${ }^{\mathrm{var}}$, benediximus $\mathrm{PEL}^{\mathrm{B}}$

nonne communicatio 5461

sanguinis christi est ] est sanguinis christi $61 \mathrm{AMst}^{\mathrm{AD}}$, corporis christi est 77 , sanguinis christi 89, sanguinis domini est AMsted, sanguinis est domini AMst $^{\text {var }}$

et $\langle 75\rangle$ ] om. $767778 \mathrm{AMst}^{\mathrm{PEL}}{ }^{\mathrm{B}}$

panis AMstrar ${ }^{\text {van }}$ pan 7778 AMsted $^{\text {ed }}$

quem frangimus $\left(88^{*}\right)$ ] frangimus AMstrar

nonne participatio 5861251 ] nonne communicatio 7576777889 AMst

corporis domini est ] corporis christi est $\mathrm{AMst}^{\mathrm{AD}}$

10:17 Present: 5154586175767778878889 251 AMst PEL

quoniam unus panis ] abs. 87, quia unus panis AMst; + et $5861 \mathrm{PEL}^{\mathrm{var}}$

unum corpus multi sumus (75) ] abs. 87

omnes PEL ${ }^{\text {var }}$ ] omnesque $54^{\mathrm{C}} 25^{\mathrm{C}} \mathrm{PEL}^{\mathrm{A}}$, nam omnes $61 \mathrm{PEL}^{\mathrm{B}}$, omnes enim

7576777889 AMst, fratres omnes 87

quidem ] qui $5154^{*} 8788 \mathrm{PEL}^{\mathrm{var}}$, om. $54^{\mathrm{C}} 617576777889{ }_{25}{ }^{\mathrm{C}}$ AMst PEL ${ }^{\mathrm{AB}}$ de uno pane ] + et de uno calice $51545^{861} 757689251 \mathrm{AMst}^{\mathrm{PEL}}{ }^{\mathrm{B}}$, + et uno calice (77) 78, + comedimus PEL var

participamur ${ }_{51} \mathrm{AMst}^{\mathrm{var}}$ ] participantur 58, percipimus 757689 , participamus uel percipimus 77, participamus 8725 AMst $^{\text {ed }}$ \pm unum corpus sumus christi 58

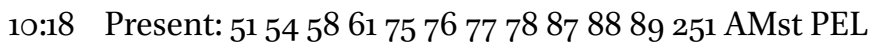

uidete ] uidite 251 ; + enim $6188^{*}$

israhel secundum carnem nonne qui 6176

edunt 61 ] manducant 75 (76) 89 AMst $^{\mathrm{AD}}$; + uel edentes 77

hostias 88 ] sacrificia $7576\left(7^{*}\right)^{\mathrm{C}} 89 \mathrm{AMst}^{\mathrm{AD}}$, sacrificia uel hostias 77

participes $51\langle 87\rangle 251$ ] participes sunt uel communicantes 77 , socii $\mathrm{AMst}^{\mathrm{AD}}$ sunt altaris 75 ] altaris sunt 7778

10:19 Present: 5154586175767778878889 251 AMst PEL SPM

quid ergo dico (75) ] quid ergo 76

quod $\mathrm{AMst}^{\text {var }}$ ] quia $757_{6} \mathrm{AMst}^{\text {ed }}$, quo 87 , quasi 89 , om. AMst $^{\mathrm{AD}}$

idolis $\left.5158 \mathrm{AMst}^{\mathrm{var}}\right]$ simulacro $7576 \mathrm{AMst}^{\text {ed }}$, idolo 89, om. AMst $\mathrm{AD}^{\mathrm{AD}}$

immolatum $5^{8}$ ] om. $\mathrm{AMst}^{\mathrm{AD}}$ 
sit aliquid 54 ] est aliquid $75767889 \mathrm{PEL}^{\mathrm{B}}$, sit est aliquid 77 , aliquid est

$\mathrm{AMst}^{\mathrm{ed}}$, aliquid sit $\mathrm{AMst}^{\mathrm{var}}$, om. AMst $^{\mathrm{AD}}$; + aut idolum sit aliquid 87 aut ] non 757677788789 AMst PEL $^{\mathrm{B}} \mathrm{SPM}$

quod AMst ${ }^{\text {var }}$ ] quia AMst ${ }^{\text {ed }}$

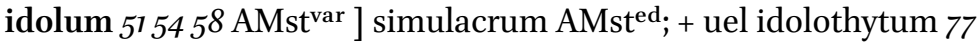
sit aliquid $5487 \mathrm{AMst}^{\mathrm{var}}$ ] fuit aliquid $78^{*}$, est aliquid $\mathrm{AMst}^{\mathrm{ed}}$

10:20 Present: 5154586175767778878889 251 AMst PEL SPM SPE sed quae 5154586176778788

immolant gentes $58 \mathrm{AMst}^{\mathrm{var}}$ ] gentes immolant 517778 , sacrificant 7576 AMst ${ }^{\text {ed }}$ SPM, immolant PEL ${ }^{\mathrm{B}}$

daemoniis $51545^{8} 617677788788^{C} 25^{1} \mathrm{SPE}^{\mathrm{var}}$ ] demones $88^{*}$, daemonibus SPE ${ }^{\text {ed }}$

immolant 5887 ] sacrificant $7576 \mathrm{sPM}$

et non deo $A M s t^{\text {var }} \mathrm{PEL}^{\mathrm{var}}$ ] et non domino $5^{8}$ 61, et non deo sacrificant $\mathrm{AMst}^{\mathrm{AD}}$, om. AMst $\mathrm{Ad}^{\mathrm{ed}} \mathrm{PEL} \mathrm{AB}^{\mathrm{AB}} \mathrm{SPM}$

nolo autem uos $\mathrm{AMst}^{\mathrm{var}}$ ] nolo uos $75768789 \mathrm{AMst}^{\mathrm{ed}} \mathrm{PEL}^{\mathrm{B}}$

socios fieri daemoniorum 51545861 (87) (88) ] daemoniorum socios fieri

75767778 , participes fieri daemoniorum AMst ${ }^{\mathrm{ed}}$, fieri socios daemoniorum $\mathrm{AMst}^{\mathrm{AD}}$, socios fieri daemonum PEL ${ }^{\mathrm{var}}$, socios esse daemoniorum SPM $^{\text {var }}$

non potestis calicem domini bibere et calicem daemoniorum 5154586176 777887

10:21 Present: $5^{1} 545^{861} 75767778878889$ 251 AMst PEL SPM SPE non potestis ] om. AMst $\mathrm{AD}^{\mathrm{AD}}$

mensae domini $5154^{c} 5876788788$ ] mensa domini $54^{*}$, mensaeque domini $\mathrm{AMst}^{\mathrm{AD}}$

participes esse 5488251 ] participare 7576788789 AMst $^{\mathrm{AD}}$ SPM, participare uel participari 77 , participari $88^{\text {alt }}$, communicare AMst ${ }^{\text {ed }}$ et mensae daemoniorum 515458617677788788

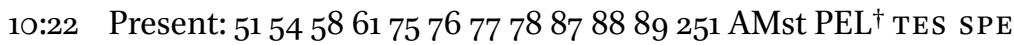
an (87) $\mathrm{AMst}^{\text {var }} \mathrm{PEL}^{\mathrm{var}}$ ] aut $757689 \mathrm{AMst}^{\mathrm{ed}}$, an uel aut 77 , ut $\mathrm{AMst}^{\mathrm{A}}$, ipsi PELgl, abs. TES; + domino PEL $^{\mathrm{B}}$

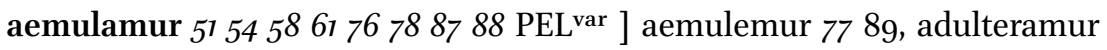
$\mathrm{PEL}^{\text {var }}$, adulamur PEL ${ }^{\text {var }}$, me zelauerunt $\mathrm{PEL}^{\mathrm{gl}}$, abs. TES

$\dagger{ }_{1}$ Cor. 10:22. Pelagius notes an alternative reading, which is indicated as PEL ${ }^{\text {gl }}$. 


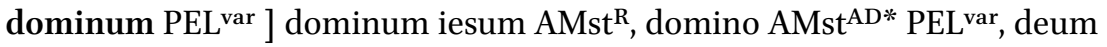
PEL ${ }^{\mathrm{A}}$, in non deo PEL ${ }^{\mathrm{gl}}$, om. $\mathrm{PEL}^{\mathrm{B}}$, abs. TES

numquid fortiores 587788 ] abs. TES

illo sumus ] illos sumus 87 , abs. TES

omnia licent 61 ] omnia mihi licent $5^{1} 5^{8} 88^{*} \mathrm{AMst}^{\mathrm{R}} \mathrm{TES}^{\mathrm{var}} \mathrm{SPE}^{\mathrm{var}}$, om. 78 PEL $^{\mathrm{B}}$

sed non omnia ] om. $5878 \mathrm{PEL}^{\mathrm{B}}$; + mihi $\mathrm{AMst}^{\mathrm{R}}$

expediunt ] aedificant 5 , om. $5878 \mathrm{PEL}^{\mathrm{B}}$

10:23 Present: $5^{1} 545^{8} 61757678878889251$ AMst PEL TES SPE omnia licent 61 ] omnia mihi licent $5_{1} 88^{*} \mathrm{SPE}^{\mathrm{var}}$, om. $5^{8}$ sed non omnia aedificant 54587688 ] sed non omnia expediunt 5178

10:24 Present: $5^{1} 545^{8} 6175767778878889$ 251 AMst PEL SPE nemo quod suum est ] nemo quod est 58 , om. AMst ${ }^{\text {var }}$ quaerat 5158788788 ] quo erat $54^{*}$, quaerit 7576 , om. AMstvar sed quod alterius ] om. AMstvar

10:25 Present: $5^{1} 545^{8} 6175767778878889$ 251 AMst PEL sPE omne quod ] omnia quae $61 \mathrm{PEL}^{\mathrm{B}}$, omnem quod 87 in macello 54 ] in macellum 7576 uenit ] ueniunt $61 \mathrm{PEL}^{\mathrm{B}}$, uenit id est uenditur id est a $\mathrm{u}[\ldots]$ ueneo 77 , ueniat 78 , uenditur 87 , uenundatur 89 , ueneunt PEL ${ }^{\text {ed }}$ manducate nihil $5154 \mathrm{AMst}^{\mathrm{AD}}$ ] edite nihil $7778 \mathrm{AMst}^{\mathrm{ed}}$ interrogantes ] disquaerentes $\mathrm{AMst}^{\mathrm{var}}$, discernentes $\mathrm{AMst}^{\mathrm{AD}}$ propter conscientiam 58

10:26 Present: $5^{1} 545^{8} 6175767778878889$ 251 AMst PEL CAr SPE ${ }^{\dagger}$ domini est $\mathrm{AMst}^{\mathrm{AD}}$ ] est enim $61 \mathrm{AMst}^{\text {ed }} \mathrm{PEL}^{\mathrm{A}}$, enim est $757678 \mathrm{AMst}^{\mathrm{var}}$, enim 77 terra et plenitudo eius

10:27 Present: $51545^{8} 6175767778878889$ 251 AMst PEL sPE ${ }^{\dagger}$ si quis uocat uos ] si quis autem uocat uos $\mathrm{AMst}^{\mathrm{AD}}$ infidelium ] ex infidelibus 617576777889 AMst PEL var \pm ad cenam $51545^{8} 617576777889$ AMst PEL $^{\mathrm{B}}$ et uultis ire $\mathrm{AMst}^{\mathrm{var}}$ ] et uolueritis ire 7778 , et uult e ire 87 , et itis $89 \mathrm{AMst}^{\mathrm{ed}}$, et ire uolueritis $\mathrm{AMst}^{\mathrm{AD}}$, et ibitis $\mathrm{PEL}^{\mathrm{B}}$

$\dagger \quad$ 1Cor. 10:26-27. These verses are omitted from SPE due to eyeskip. 


\section{omne quod}

uobis adponitur $5^{1} 5^{8} 6187$ ] ante uos ponitur 7589 , ponitur ante uos 76 , adpositum fuerit uobis 7778 , adpositum uobis fuerit $\mathrm{AMst}^{\text {ed }}$, adponitur ante uos $\mathrm{AMst}^{\mathrm{AD}}$, adpositum fuerit $\mathrm{AMst}^{\mathrm{var}}$, adponitur uobis $P E L^{B}$

manducate $\mathrm{AMst}{ }^{\mathrm{AD}}$ ] edite $7778 \mathrm{AMst}^{\text {ed }}$ nihil interrogantes 5154 ] nihil disquirentes AMst ${ }^{\mathrm{ed}}$, nihil discernentes $\mathrm{AMst}^{\mathrm{AD}}$

propter conscientiam $\left.545^{8}\left(25^{1}\right) \mathrm{AMst}^{\mathrm{ADR}}\right]$ om. AMst $\mathrm{od}^{\mathrm{ed}}$

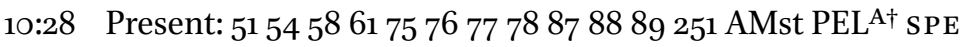

si quis autem $A M s t^{\text {var }}$ ] si autem quis 77 , si quis [3-5] 88*, si uero aliquis AMst $^{\mathrm{ed}}$; + uobis AMst ${ }^{\mathrm{AD}}$

dixerit hoc ] hoc 78

immolaticium $58 \mathrm{AMst}^{\text {ed }}$ ] immolatum $54 \mathrm{AMst}^{\mathrm{AD}} \mathrm{PEL}^{\mathrm{var}} \mathrm{SPE}^{\mathrm{var}}$, sacrificatum 61, immolatium $88 P E L^{\text {var }}$, immolantium $\mathrm{SPE}^{\mathrm{var}}$

est idolis $5^{1} 5^{8}$ (88) $\mathrm{PEL}^{\mathrm{var}}$ ] est 7576 (78) 89 AMst $^{\text {ed }} \mathrm{PEL}^{\mathrm{A}}$, idolis est 77 , et AMst $^{\text {var }}$

nolite manducare ] nolite edere uel ne edite 77

propter illum qui (88) $\mathrm{AMst}^{\mathrm{R}} \mathrm{PEL}^{\text {var }}$ ] om. $7778 \mathrm{AMst}^{\mathrm{ed}} \mathrm{PEL}^{\mathrm{A}}$

indicauit $\mathrm{AMst}^{\mathrm{R}} \mathrm{PEL}^{\text {var }}$ ] manducauit 58 , manducabit $61^{*}$, iudicabit $61^{\mathrm{C}}$, nuntiauit 75 76, om. 7778 AMst $^{\text {ed }}$ PEL $^{\mathrm{A}}$

et propter $\mathrm{AMst}^{\mathrm{R}} \mathrm{PEL}^{\text {var }}$ ] propter $75767778 \mathrm{AMst}^{\text {ed }} \mathrm{PEL}^{\mathrm{A}}$

conscientiam $545^{8}$ ] [2-4] conscientiam 88*; + eius PEL ${ }^{\text {var }}$

10:29 Present: $5^{1} 545^{8} 6175767778878889$ 251 AMst PEL SPE

conscientiam autem 5888251 ] consciam autem 87

dico non tuam (87) ] non dico tuam AMst ${ }^{\text {var }}$

sed alterius ut quid enim libertas 5478

mea ] nostra 61

iudicatur ] iudicabitur 78 , iudicetur 88

ab alia conscientia $54 \mathrm{AMst}^{\mathrm{var}} \mathrm{PEL} \mathrm{L}^{\mathrm{var}}$ ] ab aliena conscientia $5^{1} 5^{8} \mathrm{AMst}^{\mathrm{var}}$,

ab infideli conscientia 6175767789 AMst $^{\text {ed }}$ PEL $^{\mathrm{AB}}$

10:30 Present: 5154586175767778878889 251 AMst PEL sPE

si AMst ${ }^{\text {var }}$ ] si enim $5^{1} 5^{8} 78$ 251, si ergo $618788^{\mathrm{C}} 89 \mathrm{AMst}^{\mathrm{ed}} \mathrm{PEL}^{\mathrm{B}}$

ego AMst ${ }^{\text {var }}$ ] om. $618788^{\mathrm{C}} 89$ AMst $^{\text {ed }}$ PEL $^{\mathrm{B}}$

cum AMst ${ }^{\mathrm{var}}$ ] om. 75767789 AMst $^{\text {ed }}$

$\dagger{ }_{1}$ Cor. 10:28. The whole verse is missing from $\mathrm{PEL}^{\mathrm{B}}$. 
gratia 5825 AMst $^{\text {var }}$ ] gratiam 89 , gratiae AMst ${ }^{\text {ed }}$

participo $\mathrm{PEL}^{\mathrm{var}}$ ] participor $58 \mathrm{PEL}^{\mathrm{B}}$, percipio $757689 \mathrm{AMst}^{\mathrm{var}} \mathrm{PEL}^{\mathrm{A}}$, particeps sum AMst ${ }^{\text {ed }}$

quid ] quid adhuc $61 \mathrm{PEL}^{\mathrm{B}}$, cur 251, quare adhuc PEL var

blasphemor 6178 ] blasphemur 87 , blasphemamur PEL var

pro eo quod AMst ${ }^{\text {var }}$ ] pro quo ego $757677 \mathrm{AMst}^{\text {ed }}$, pro quo 89

gratias ago 251] bene gratulor uel gratias ago 77

10:31 Present: $5^{1} 545^{861} 75767778878889$ 251 AMst PEL SPM SPE

siue ] si $7576 \mathrm{AMst}^{\mathrm{D}}$

ergo AMst ${ }^{\text {var }}$ SPM $^{\text {var }}$ ] uero ergo 7576 , igitur AMst ${ }^{\text {ed }}$, om. sPM $^{\text {ed }}$

manducatis siue (87) AMst $^{\text {var }}$ ] editis siue AMst ${ }^{\text {ed }}$

bibitis 75 ] bibetis 6187251 AMst $^{\text {var }} \mathrm{SPM}^{\mathrm{var}}$, bibatis SPM${ }^{\mathrm{var}}$

uel SPM ${ }^{\text {var }}$ ] siue 54617576 (77) 89 AMst PEL $^{\mathrm{B}}$ SPM $^{\text {ed }}$, aut 87

aliud quid facitis $5487251 P E L^{\text {var }} \mathrm{SPM}^{\mathrm{var}}$ ] aliquid faciatis 61, aliquid facitis 75 7689 AMst $^{\text {ed }} \mathrm{PEL}^{\mathrm{AB}}$, faciatis aliquid 77 , aliud aliquid facitis $\mathrm{AMst}^{\mathrm{var}}$, facitis aliquid $A M s t^{A D} S_{P M}{ }^{e d}$, aliquid quod facitis PEL ${ }^{\text {var }}$, aliud quod facitis $\mathrm{SPM}^{\mathrm{var}}$

omnia in gloriam dei ] omnia in dei gloriam 58 , omnia in gloria dei $8725^{1}$ $\mathrm{AMst}^{\mathrm{var}} \mathrm{PEL}^{\mathrm{B}}$

facite $87 \mathrm{AMst}^{\mathrm{var}} \mathrm{SPM}^{\mathrm{var}}$ ] facitis 7576 , om. $7778 \mathrm{AMst}^{\mathrm{ed}} \mathrm{PEL}^{\mathrm{B}} \mathrm{SPM}^{\mathrm{ed}}$

10:32 Present: $51545^{8} 61757677788889$ AMst PEL SPE

sine offensione estote $\left.\left(\mathrm{PEL}^{\mathrm{A}}\right)\right]+$ et $7576 \mathrm{AMst}^{\mathrm{A}}$

iudaeis et 51545861767888

gentilibus ] gentibus $5^{861} 7888 \mathrm{PEL}^{\mathrm{var}} \mathrm{SPE}$, graecis $75767789 \mathrm{AMst}^{\mathrm{PEL}} \mathrm{AB}^{\mathrm{AB}}$ et ecclesiae dei 51545861767888 ] et ecclesia dei $\mathrm{SPE}^{\mathrm{var}}$

10:33 Present: $51545^{8} 617576777888$ 89 AMst PEL SPE

sicut et ego $\mathrm{AMst}^{\mathrm{var}}$ ] et sicut et ego 77 , sicut ego AMst ${ }^{\text {ed }}$

per omnia omnibus ] omnibus per omnia $6175767889 \mathrm{AMst}^{\mathrm{PEL}}{ }^{\mathrm{B}}$, omnibus

per uel secundum omnia 77 , post omnia omnibus $\mathrm{SPE}^{\mathrm{var}}$

placeo ] placebo PELvar

non quaerens quod mihi 515458767888 ] + uel meum 77

utile est sed quod ] utile est sed AMst ${ }^{\text {var }}$

multis ut salui fiant

11:1 Present: $5^{1} 545^{8} 617576777888$ 89 AMst PEL CAr SPE

imitatores mei estote 5461 ] + fratres CAr

sicut et ego christi $\left(88^{*}\right)$ 
11:2 Present: $5^{1} 545^{8} 617576777888$ 89 AMst PEL

laudo autem uos ] laudo uos autem $\mathrm{PEL}^{\mathrm{B}}$, laudo enim uos $P E L^{\text {var }}$

fratres ] om. AMst ${ }^{\mathrm{AD}}$

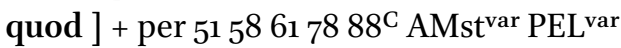

omnia ] omnium PEL ${ }^{\mathrm{B}}$; + [...] $54^{*}$

mei AMst ${ }^{\text {var }}$ PEL $^{\text {var }}$ ] mea 757689 AMst $^{\text {ed }}$ PEL $^{\mathrm{A}}$, meorum PEL $^{\mathrm{B}}$

memores estis AMst ${ }^{\mathrm{var}}$ ] memoria tenetis 75 76, memoramini 77 , memoria retinetis $89 \mathrm{AMst}^{\mathrm{ed}}$

et sicut $\mathrm{AMst}{ }^{\mathrm{AD}}$ ] et quomodo $\mathrm{AMst}^{\mathrm{ed}}$; + ubique $75777^{\mathrm{gl}} 89 \mathrm{AMst}^{\mathrm{ed}} \mathrm{PEL}^{\mathrm{B}}$

tradidi $\mathrm{AMst} \mathrm{AD}^{\mathrm{AD}}$ ] trado $\mathrm{AMst}^{\mathrm{ed}} \mathrm{PEL}^{\mathrm{B}}$, om. AMst $^{\mathrm{var}}$

uobis $\mathrm{AMst}^{\mathrm{AD}}$ ] om. $7789 \mathrm{AMst}^{\text {ed }} \mathrm{PEL}^{\mathrm{B}}$

praecepta mea 515458617888 ] traditionem meam 7576 , traditiones meas

7789 AMst

tenetis ] ne tenetis 77

11:3 Present: $5^{1} 545^{8} 61757677788889$ AMst PEL TES FOR

uolo autem $\mathrm{AMst}^{\mathrm{AD}}$ ] uolo $7789 \mathrm{AMst}^{\mathrm{ed}}$, abs. TES FOR

uos scire quod omnis $\mathrm{AMst}^{\mathrm{AD}}$ ] uos scire quia omnis $757677 \mathrm{AMst}^{\mathrm{ed}}$, abs.

TES FOR

uiri caput ] caput uiri TES FOR

christus est ] est christus $5^{8} \mathrm{AMst}^{\mathrm{var}}$, christi est 75 , est christi PEL ${ }^{\text {var }}$, christus TES FOR

caput autem 89 ] abs. TES FOR; + christi 77

mulieris uir ] abs. TES FOR

caput uero $\mathrm{AMst}^{\mathrm{AD}}$ ] caput autem $77 \mathrm{AMst}^{\mathrm{ed}} \mathrm{PEL}^{\mathrm{B}}$, caput FOR, abs. TES

christi deus ] abs. TES

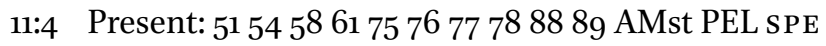

omnis uir orans ] omnis autem uir orans PEL var

aut prophetans 61 ] et prophetans 88 , uel prophetans 89

uelato capite ] supra caput habens uelamen 61, uelatum caput habens

77

deturpat ] deturpet 61, dehonestat $\mathrm{AMst}^{\mathrm{AD}}$

caput suum 89

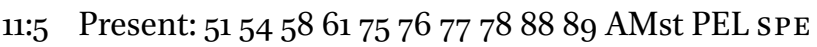

omnis autem mulier orans ] omnis mulier orans $\mathrm{SPE}^{\mathrm{var}}$

aut ] uel $757677 \mathrm{AMst}^{\mathrm{AD}}$

prophetans 61 ] prophetizans $757^{6} \mathrm{C}$

non uelato capite ] non uelato $54^{*}$, inuelato capite $S P E^{\text {var }}$ 
deturpat $88^{*}\left(\mathrm{PEL}^{\mathrm{A} *}\right)^{\mathrm{C}}$ ] deturpeat 61 , dehonestat $89 \mathrm{AMst}^{\mathrm{AD}} \mathrm{PEL}^{\mathrm{B}}$, confundit AMst ${ }^{\text {ed }}$; uel dehonestat 77

caput $\operatorname{suum}\left(76^{*}\right) 89$

unum est enim ] unum enim est 77 , abs. SPE

atque ] ac $51586178 \mathrm{PEL}^{\mathrm{var}}$, at $5488^{*}$, et ipsum atque $757^{6} 89$, atque idipsum $77 \mathrm{AMst}^{\mathrm{ed}}$, et idipsum ac $\mathrm{AMst}^{\mathrm{AD}}$, abs. SPE

si decaluetur ] decaluata sit 7576 , si decaluata sit 77 , decaluata 89 , ut decaluata $\mathrm{AMst}^{\mathrm{ed}}$, si sit decaluata $\mathrm{AMst}^{\mathrm{AD}}$, ut decaluetur $\mathrm{AMst}^{\mathrm{var}}$, abs. SPE

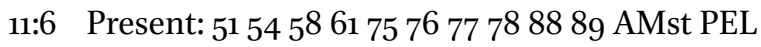

nam si ] si enim $757677 \mathrm{AMst}^{\mathrm{AD}}$, quod si 89 , si autem $\mathrm{AMst}^{\text {ed }}$

non uelatur mulier

et tondeatur ] tondeatur $5^{1} 54^{\mathrm{C}} 5^{8} \mathrm{AMst}^{\mathrm{ed}} \mathrm{PEL}^{\mathrm{B}}$, [2] t[1]deatur $54^{*}$, et tundeatur 88 , tundatur $\mathrm{AMst} \mathrm{AD}^{\mathrm{AD}}$

si uero ] quod si $757689 \mathrm{AMst}^{\mathrm{AD}}$, si autem $\mathrm{AMst}^{\text {ed }}$

turpe est mulieri tonderi ] turpe est tonderi mulieri 58 , turpe est mulieri tondere $61 \mathrm{AMst}^{\mathrm{var}}$, turpe est mulieri tunderi 88 , turpe est mulieri decaluari $\mathrm{PEL}^{\mathrm{B}}$, mulieri turpe est tondere $\mathrm{PEL}^{\mathrm{var}}$

aut decaluari ] uel decaluari 77 , aut detonderi $P E L^{B}$

uelet caput suum $\mathrm{AMst}^{\mathrm{AD}}$ ] uelet caput uel uelet se 77 , uelet caput $89 \mathrm{AMst}^{\mathrm{ed}}$ PEL $^{\mathrm{B}}$

11:7 Present: 515458617576777888 89 AMst PEL

uir quidem ] uir enim $7576 \mathrm{AMst}$

non debet uelare caput 89$]+$ suum $58617888 \mathrm{PEL}^{\mathrm{var}}$

quoniam ] cum $7576 \mathrm{AMst}^{\mathrm{AD}}$, cum sit (89) $\mathrm{AMst}^{\mathrm{ed}}$

imago et gloria $51545^{8}$ ] gloria $\mathrm{AMst} \mathrm{AD}^{\mathrm{AD}}$

est dei ] dei sit $7576 \mathrm{AMst}^{\mathrm{AD}}$, dei $7789 \mathrm{AMst}^{\mathrm{ed}} \mathrm{PEL}^{\text {var }}$, dei est 78 ; + a principio uel per initium 77

mulier autem ] mulier enim $\mathrm{AMst}^{\mathrm{AD}}$

gloria uiri est ] uiri gloria est 58 , gloria est uiri $88 \mathrm{PEL}^{\mathrm{B}}$

11:8 Present: 51545861757677788889 AMst PEL

non enim ] non enim est 757677 89, non est $A M s t^{\text {ted }}$, non est enim AMst ${ }^{\text {tar }}$ uir ] om. 61

ex muliere est ] de muliere $757689 \mathrm{AMst}^{\mathrm{AD}}$, ex muliere $77 \mathrm{AMst}^{\mathrm{ed}}$ sed mulier ex uiro ] sed mulier propter uirum 7576 , sed mulier de uiro 89 $\mathrm{AMst}^{\mathrm{AD}}$ 
11:9 Present: $5^{1} 545^{8} 617576777888$ 89 AMst PEL

etenim non est ] non est $\mathrm{AMst}^{\mathrm{AD}}$

creatus ] om. PEL var

uir ] homo 89 PEL $^{B}$

propter mulierem sed mulier propter uirum (78)

11:10 Present: $5^{1} 545^{8} 617576777888$ 89 AMst PEL

ideo ] propter hoc $757689 \mathrm{AMst}^{\mathrm{AD}}$, propterea uel ideo 77 , propterea $\mathrm{AMst}^{\mathrm{ed}}$, et ideo PELvar

debet mulier ] mulier debet $\mathrm{AMst}^{\text {var }}$ PEL var

potestatem habere ] uelamen habere $5^{8} \mathrm{AMst}^{\mathrm{R}}$

supra caput ] super caput $777889 \mathrm{AMst}^{\mathrm{var}} \mathrm{PEL}^{\mathrm{B}}$

\pm et 51 , suum 58 , etiam AMst ${ }^{R}$

propter angelos 61

11:11 Present: $5^{1} 545^{8} 617576777888$ 89 AMst PEL

uerumtamen neque 5154

uir sine muliere ] mulier sine uiro 75767778 89, muliere sine uiro AMst

neque ] nec AMstrar

mulier sine uiro ] uir sine muliere 7576777889 AMst

in domino ] in deum PELvar

11:12 Present: $5^{1} 545^{8} 61757677788889$ AMst PEL

nam sicut mulier ] sicut enim mulier $\mathrm{AMst}^{\mathrm{AD}}$

de ] ex 617576

uiro ] + est 61 AMst $^{\text {var }}$

ita et uir ] ita et 54 , sic et uir $\mathrm{AMst}^{\mathrm{AD}}$

per ] ex $61^{*}$, de 89

mulierem $\left.\left(78^{*}\right)\right]+$ uir $54,+$ est 61

omnia autem ex deo

11:13 Present: $5^{1} 545^{8} 617576777888$ 89 AMst PEL

uos ipsi ] in uobis ipsis 54, inter uos ipsos 77, apud uosmetipsos AMst ${ }^{\mathrm{AD}}$ iudicate decet $(76)\left(78^{*}\right)$ ] iudicate dece[1] $54^{*}$, iudicate decetne $\mathrm{AMst}^{\mathrm{R}}$ mulierem non uelatam ] mulierem non uelato capite $89 \mathrm{AMst}^{\mathrm{AD}}$ orare deum ] orare dominum AMst ${ }^{\text {var }}$

11:14 Present: $5^{1} 545^{8} 617576777888$ 89 AMst PEL

nec ] nonne autem 77 , om. AMst ${ }^{\text {var }}$

ipsa natura docet uos ] ipsa natura uos docet $5^{1} \mathrm{AMst}^{\mathrm{R}}$ 
quod $\mathrm{AMst} \mathrm{AD}^{\mathrm{AD}}$ ] om.51, quoniam $5^{8} 88^{\mathrm{C}}$, quia $75767789 \mathrm{AMst}^{\mathrm{ed}} \mathrm{PEL}^{\mathrm{var}}$, qui PELvar

uir quidem si comam 54 ] uir quidam si comam $78^{*} \mathrm{PEL}^{\mathrm{var}}$ nutriat AMst ${ }^{\text {var }}$ ] habeat 61, nutriat uel habeat 77, habuerit AMst ${ }^{\text {ed }}$ ignominia ] om. AMst ${ }^{\text {var }}$ est illi ] illi est 77, om. AMst ${ }^{\mathrm{var}}$

11:15 Present: $51545^{8} 61757677788889$ AMst PEL

mulier uero ] mulier autem 6175767789 AMst, mulier h $78^{*}$, mulier $78^{\mathrm{C}}$ si comam $54(78)$ ] si capillos $\mathrm{PEL}^{\mathrm{B}}$ nutriat (89) ] habeat $61 \mathrm{AMst}^{\mathrm{AD}} \mathrm{PEL}^{\mathrm{B}}$, habuerit $\mathrm{AMst}^{\mathrm{ed}} ;+[\ldots] 61^{*}$ gloria est illi ] gloria illi est 77 quoniam ] quoniam quidem 61 $\mathrm{PEL}^{\mathrm{B}}$, quia $75767789 \mathrm{PEL}^{\mathrm{A}}$, quae $\mathrm{AMst}^{\mathrm{AD}}$, omnes AMst ${ }^{\mathrm{var}}$

capilli pro uelamine $\left(88^{*}\right)$ AMst $\left.{ }^{\text {var }}\right]$ coma pro uelamine $757677\left(89^{*}\right)^{\mathrm{C}}$ AMst $^{\text {ed }}$

ei dati sunt $A M s t^{\text {var }}$ ] est 757689 , data est ei 77 , data est $A M s t^{e d}$, sunt $P E L^{B}$, dati sunt ei PEL var

11:16 Present: 51545861757677788889 AMst PEL CAr si quis autem ] si autem quis 77 uidetur contentiosus esse nos 54586188 talem ] tal[2] $54^{*}$ consuetudinem non habemus neque 61 ] consuetudinem non habemus CAr ecclesiae dei $617677 \mathrm{PEL}^{\mathrm{var}}$ ] ecclesia dei $51545^{8} 788889 \mathrm{AMst}^{\mathrm{var}} \mathrm{PEL}^{\mathrm{AB}}$, abs. CAr

11:17 Present: $5^{1} 545^{8} 61757677788889$ AMst PEL SPE

hoc autem

praecipio 5154587888 ] praecipiens 617576 , praecipiens uel adnuntians 77 , pronuntio $\mathrm{AMst}^{\mathrm{AD}}$, principio $\mathrm{AMst}^{\mathrm{var}}$, pretio $\mathrm{PEL}^{\mathrm{B}}$

non laudans ] non laudo 6175767789

quod non in melius ] quod non melius $\mathrm{AMst}^{\mathrm{A}}$ sed in deterius $\mathrm{AMst}^{\mathrm{var}}$ ] sed in peius $\mathrm{AMst}^{\mathrm{ed}}$ conuenitis ] uenistis 89

11:18 Present: $51545^{8} 61757677788889$ AMst PEL SPE primum quidem $\mathrm{AMst}^{\mathrm{var}}$ ] primum enim $\mathrm{AMst}^{\mathrm{ed}}$ conuenientibus ] uenientibus 78 uobis ] + in unum SPEvar 
in ecclesia $6176 \mathrm{AMst}^{\mathrm{var}}$ ] in ecclesiam $54^{*} 88 \mathrm{AMst}^{\mathrm{ed}} \mathrm{PEL}^{\mathrm{var}} \mathrm{SPE}^{\mathrm{var}}$; + dei 58 audio

scissuras AMst ${ }^{\mathrm{var}}$ ] scisma 61 , scissurae 75 , scismata $7778 \mathrm{AMst}^{\mathrm{AD}} \mathrm{PEL}^{\mathrm{B}}$, dissensiones AMsted

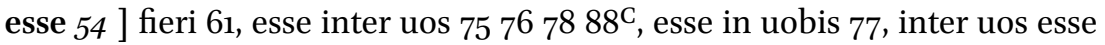
$S P E^{\text {var }}$

et ] om. PEL var

ex parte credo AMstrar ] ex parte aliqua credo $75\langle 76\rangle 7789 \mathrm{AMst}^{\mathrm{AD}}$, partim credo AMst ${ }^{\text {ed }}$

11:19 Present: $51545^{8} 61757677788889$ AMst PEL tes SPE

nam oportet (78) ] oportet enim 75767789 AMst SPE ${ }^{\text {var }}$, oportet TES

et $\mathrm{SPE}^{\mathrm{var}}$ ] om. $5^{1} 546188^{\mathrm{C}} \mathrm{PEL}^{\mathrm{var}} \mathrm{TES}^{\mathrm{var}} \mathrm{SPE}^{\text {ed }}$

hereses $6 r(75) 7677 \mathrm{TES}^{\mathrm{var}}$ ] heresis $\mathrm{TES}^{\mathrm{ed}}$; + in uobis AMst ${ }^{\mathrm{AD}}$

esse ] fieri $\mathrm{TES}^{\mathrm{var}}$

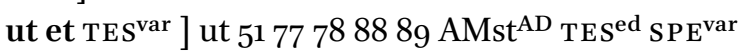

qui probati sunt TES $S^{\mathrm{var}}$ ] qui probati $54^{*}$, probati $7576777889 \mathrm{AMst}^{\mathrm{AD}} \mathrm{TES}^{\mathrm{ed}}$ manifesti fiant $\mathrm{TES}^{\mathrm{var}}$ ] manifesti sint $\mathrm{TES}^{\mathrm{ed}}$

in uobis ] inter uos 757689 AMst, in nobis TES ${ }^{\mathrm{var}}$, in uos TES ${ }^{\mathrm{var}}$

11:2O Present: 51545861757677788889262 AMst PEL SPE

\pm primum quidem 61 , fratres 262

conuenientibus ] conuentibus 262

ergo uobis ] uobis $6175767789 \mathrm{AMst}^{\mathrm{AD}} \mathrm{PEL}^{\mathrm{B}}$, igitur uobis $\mathrm{AMst}^{\mathrm{var}}$, uobis igitur AMsted

in unum ] in id ipsum uel in unum 77

iam non est ] iam non $617789 \mathrm{AMst}^{\mathrm{var}}$, non 7576

dominicam cenam manducare 61777889 SPE

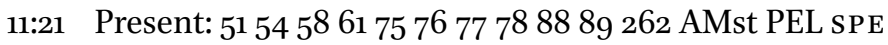

unusquisque enim ] unusquisque AMst ${ }^{\text {var }}$

suam $\mathrm{AMst}^{\mathrm{AD}}$ ] uestrum propriam AMsted

cenam praesumit $5154586176(77) 788889\langle S P E\rangle]$ ] cenam praesumat 262 PEL ${ }^{\text {var }}$

ad manducandum AMst ${ }^{\mathrm{var}}$ ] in manducando $7589 \mathrm{AMst}^{\mathrm{ed}}$, in manducandum 77

et alius quidem esurit 6177

alius autem AMstrar $]$ alius quidem 262, alius AMsted, et alius AMst ${ }^{\mathrm{R}}$; + quidem PEL var

ebrius est ] + uel ebriatur 77 
11:22 Present: $5^{1} 545^{8} 61757677788889262$ AMst PEL sPE

numquid domos non habetis 6177 (262)

ad manducandum AMst ${ }^{\mathrm{var}}$ ] ut manducetis 61, manducare 77, ad edendum $\mathrm{AMst}^{\mathrm{ed}}$

et bibendum $\langle 76\rangle$ ] et ad bibendum 58 , et bibatis 61 , et bibere 77

aut ecclesiam dei 5161262

contemnitis $5154^{c} 5^{8} 6188^{c} 89$ ] confunditis AMst ${ }^{\mathrm{var}}$, contaminatis PEL var et confunditis ] et confundetis AMstrar $\mathrm{SPE}^{\mathrm{var}}$

eos qui non habent 262 ] eos qui non habentur 54, non habentes 757689

AMst, qui non habetis uel non habentes 77

quid dicam uobis laudo uos

in hoc non laudo ] sed in hoc non laudo $5^{8}$

11:23 Present: $51545^{8} 6175767778848889262$ AMst PEL

ego enim ] et ego enim 58 , ego autem $61 \mathrm{PEL}^{\text {var }}$, fratres ego 84

accepi a domino $6175^{c} 84$ ] accepi domino 54 , accepi a deo $75^{*} 77$

quod et ] abs. 84, quod etiam $\mathrm{AMst}^{\mathrm{D}}$, quod PEL ${ }^{\text {var }}$

tradidi uobis ] abs. 84, uobis tradidi PEL var

quoniam ] quia $7589 \mathrm{AMst}^{\mathrm{AD}}$, quod $77, a b s .84$

dominus iesus ] dominus noster iesus christus $5_{1}$ PEL var, dominus iesus christus $58 \mathrm{AMst}^{\mathrm{AD}}$, abs. 84

in qua nocte tradebatur ] abs. 84

accepit panem ] accepit autem panem $54^{*}$, accipit panem 61, abs. 84

11:24 Present: $5^{1} 545^{8} 61757677788889262$ AMst PEL

et ] om. PEL var

gratias agens ] gratias egit et $6189 \mathrm{PEL}^{\mathrm{B}}$, gratias cum egisset 7576 , benedixit et $\mathrm{AMst}^{\mathrm{AD}}$; + benedixit ac 58

fregit et dixit ] fregit dicens $\mathrm{AMst}{ }^{\mathrm{AD}}$

\pm accipite et manducate $5^{1} 5^{8262} \mathrm{AMst}^{\mathrm{R}} P E L^{\text {var }}$, accipite et manducare $88^{\mathrm{C}}$ hoc est corpus meum ] hoc meum est corpus 77

pro uobis ] quod pro uobis tradetur $5^{1} 545^{8} 7888262 \mathrm{AMst}^{\mathrm{R}} \mathrm{PEL}^{\mathrm{B}}$, quod tradidi pro uobis 61, quod pro uobis frangitur $7576 \mathrm{AMst}^{\mathrm{ed}} \mathrm{PEL}^{\mathrm{var}}$, quod pro uobis frangetur $777^{\mathrm{gl}}$, quod pro uobis confringetur 89 , quod pro uobis est $\mathrm{AMst}{ }^{\mathrm{AD}}$, quod pro uobis traditur PEL var

hoc facite ] + quotienscumque feceritis $54^{c}$

in meam commemorationem $58(75)]$ in commemorationem meam 89 , in mei commemorationem AMst, in mea commemoratione PEL var 
11:25 Present: 51545861757677788889262 AMst PEL

similiter AMst ${ }^{\text {var }}$ ] simili modo AMsted; + autem 54

et calicem postquam cenauit dicens $6 r(75) 767778262$ ] et calicem post cenam dicens 89 , et calicem postquam cenatum est dicens AMst

hic calix $\mathrm{AMst}^{\mathrm{AD}}$ ] hic est calix $617_{2}{ }_{262}$, his est calix 75 , hoc poculum AMsted, poculum AMstrar

nouum testamentum ] noui testamenti $61 \mathrm{AMst}^{\mathrm{AD}}$

est ] om. 61262

in meo sanguine ] in meo sanguinem $89^{*}$, in meum sanguinem $89^{\mathrm{C} *}$

hoc facite ] hoc facit 76

quotienscumque 54617788

bibetis AMst ${ }^{\mathrm{A}}$ ] biberitis $5^{8} 262 \mathrm{AMst}^{\mathrm{R}} \mathrm{PEL}^{\mathrm{var}}$, bibitis $75767889 \mathrm{AMst}^{\mathrm{ed}}$ $\mathrm{PEL}^{\mathrm{B}} ;+$ eum 61

in meam ] in mei AMst

commemorationem $5^{8}(88)$ ] recordationem uel commemorationem 77 , commemoratione $\mathrm{AMst}{ }^{\mathrm{AD}}$

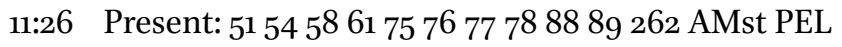

quotienscumque 5461777888 ] quotiensque $76^{*}$, om. AMsted, quotiens $\mathrm{AMst}^{\mathrm{ADR}}$

enim $\mathrm{AMst}^{\mathrm{R}}$ ] om. $61 \mathrm{AMst}^{\mathrm{ed}} \mathrm{PEL}^{\text {var }}$

manducabitis $\mathrm{AMst}^{\mathrm{AD}}$ ] manducatis $77^{*}$, manducaueritis $262 \mathrm{PEL}^{\mathrm{var}}$, om. $\mathrm{AMst}^{\mathrm{ed}}$, comederitis $\mathrm{AMst}^{\mathrm{R}}$

panem hunc et $\left.\mathrm{AMst}^{\mathrm{ADR}}\right]$ om. AMst $^{\mathrm{ed}}$

calicem $\mathrm{AMst}^{\mathrm{AD}}$ ] calicem istum $61262 \mathrm{PEL}^{\mathrm{var}}$, om. AMsted, de poculo $\mathrm{AMst}^{\mathrm{R}}$

bibetis AMst ${ }^{\mathrm{AD}}$ ] bibitis 757789 , biberitis $262 \mathrm{AMst}^{\mathrm{R}}$, om. AMst $^{\mathrm{ed}}$ mortem domini ] mortem christi 61

adnuntiatis 7789 ] adnuntiabitis $51545861767888262 \mathrm{AMst}^{\mathrm{ADR}} P E L^{B}$, adnuntiantes AMst ${ }^{\mathrm{ed}}$, adnuntietis $\mathrm{PEL}^{\mathrm{A}}{ }^{*}$

donec ueniat ] + in claritate de caelis 262

11:27 Present: $5^{1} 545^{8} 61757677788889262$ AMst PEL CAr TES SPE

itaque quicumque ] quicumque $\mathrm{TES}^{\mathrm{ed}}$, qui $\mathrm{TES}^{\mathrm{var}}$

manducauerit $262 \mathrm{AMst}^{\mathrm{AD}} \mathrm{TES}^{\mathrm{var}}$ ] ederit $\mathrm{AMst}^{\mathrm{ed}} \mathrm{TES}^{\mathrm{ed}}$, ediderit $\mathrm{TES}^{\mathrm{var}}$, edent TES ${ }^{\mathrm{var}}$, comederit TES ${ }^{\mathrm{var}}$

panem $\mathrm{AMst}^{\mathrm{AD}}$ ] panem hunc $58 \mathrm{AMst}^{\mathrm{ed}}$, hunc panem 61, om. $262 \mathrm{PEL}^{\text {var }}$ uel biberit ] aut biberit $75767778 \mathrm{AMst}^{\text {ed }}$ TES $^{\text {ed }}$, et biberit 89 AMst $^{\text {var }} \mathrm{CAr}$ TES $^{\text {var }}$, om. PEL ${ }^{\mathrm{B}}$

calicem ] om. $\mathrm{PEL}^{\mathrm{B}}$, sanguinem TES $\mathrm{sar}^{\mathrm{var}}$ 
domini indigne ] om. $\mathrm{PEL}^{\mathrm{B}}$, abs. CAr reus ] domino reus $\mathrm{AMst}^{\mathrm{ed}}$, abs. CAr erit ] erit et $\mathrm{PEL}^{\mathrm{B}}$, om. $\mathrm{SPE}^{\mathrm{var}}$, abs. $\mathrm{CAr}$ corporis et sanguinis ] corpori et sanguine TES ${ }^{\mathrm{var}}$, abs. CAr domini ] om. AMst $^{\mathrm{AD}}$, christi $\mathrm{TES}^{\mathrm{var}}$, abs. CAr

11:28 Present: 51545861757677788889262 AMst PEL $^{\mathrm{A} \dagger}$ SPE probet autem ] probat autem PEL ${ }^{\mathrm{var}}$ se ipsum homo $\mathrm{AMst}^{\mathrm{D}}$ ] se homo $61262 \mathrm{AMst}^{\text {ed }} \mathrm{PEL}^{\mathrm{var}}$, se ipse homo $\mathrm{AMst}^{\mathrm{A}}$

et sic de pane ] et sic pane $54^{*}$

illo AMst ${ }^{\mathrm{R}}$ ] om. $75767789 \mathrm{AMst}^{\mathrm{ed}}$

edat ] manducet $757689 \mathrm{AMst}^{\mathrm{AD}}$, manducat $\mathrm{PEL}^{\mathrm{var}}$

et de calice bibat $\mathrm{AMst}^{\mathrm{var}}$ ] et de poculo bibat $\mathrm{AMst}^{\mathrm{ed}}$

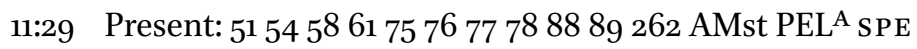

qui enim ] quicumque enim $61 \mathrm{PEL}^{\text {var }}$

manducat ] est uel manducat 77

et bibit ] et bib[1]t $54^{*}$, et bibet 75262

indigne $\left.\left(77^{*}\right) \mathrm{AMst}^{\mathrm{var}} \mathrm{PEL}^{\mathrm{var}}\right]$ om. AMst ${ }^{\text {ed }} \mathrm{PEL}^{\mathrm{A}}$

iudicium sibi ] + ipsi AMst ${ }^{\text {ed }}$

manducat et bibit non

diiudicans ] iudicans 61 PELvar, discernens 75767789 AMst

corpus ] + domini $51545^{8} 61757677788889262$ AMst PEL ${ }^{\text {var }}$ SPE $^{\mathrm{var}}$, + christi SPEvar

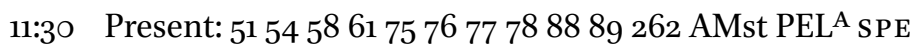
ideo ] ideoque 58 , propterea $75767789 \mathrm{AMst}^{\mathrm{AD}}$

inter uos multi $\left.\mathrm{AMst}^{\mathrm{AD}}\right]$ multi in uobis $\mathrm{AMst}^{\mathrm{ed}}$

infirmes PEL ${ }^{\mathrm{A} *}$ ] infirmi $51545861757677788889262 \mathrm{AMst}^{\mathrm{AD}} \mathrm{PEL}^{\mathrm{AC}} \mathrm{SPE}$, inualidi AMst ${ }^{\text {ed }}$

et inbecilles $67_{7} 888$ ] et aegri $757689 \mathrm{AMst}^{\mathrm{var}}$, et aegri uel inbecilles 77 , et aegroti AMsted

et dormiunt multi ] et dormiunt $\mathrm{AMst}^{\mathrm{AD}}$

11:31 Present: 51545861757677788889262 AMst PEL ${ }^{A}$ SPE

quod si ] si autem 77

$\dagger{ }_{1}$ Cor. 11:28. PEL $^{\mathrm{B}}$ jumps from 1 Cor. 11:28 to 15:3. 
nosmet ipsos $262 \mathrm{PEL}^{\text {var }}$ ] nos ipsos $75767789 \mathrm{AMst}^{\mathrm{AD}} \mathrm{PEL}^{\text {var }}$

diiudicaremus 61 PEL $^{\text {var }} \mathrm{SPE}^{\mathrm{var}}$ ] iudicaremus $75767789262 \mathrm{AMst}^{\mathrm{var}} \mathrm{PEL}^{\mathrm{var}}$ $\mathrm{SPE}^{\mathrm{ed}}$, discerneremus AMsted, diiudicaremur $P E L^{\text {var }}$

non utique ] om. PEL var

iudicaremur ] iudicaremus 78 89, diiudicaremur PEL ${ }^{\text {var }}$, om. PEL var

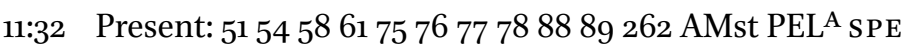

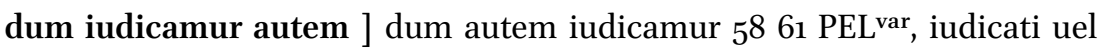
dum uel cum iudicamur autem 77, cum iudicamus autem AMst ${ }^{\text {var }}$, dum iudicamur PEL var

a domino corripimur (88) ] a domino corr[2-3]pimur $75^{*}$

ut non cum ] ne cum $7576 \mathrm{AMst}^{\mathrm{AD}}$, ut non $\mathrm{AMst}^{\mathrm{var}}$

hoc mundo damnemur $\left.515861\left(88^{*}\right)\right]$ mundo hoc damnemur 77

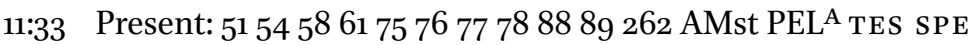

itaque fratres mei AMst ${ }^{\mathrm{var}}$ ] itaque fratres $89 \mathrm{AMst}^{\mathrm{ed}} \mathrm{PEL}^{\mathrm{var}}$, abs. TES

cum conuenitis ] conuenientes $757677 \mathrm{AMst}^{\mathrm{A}}$, dum conuenitis 262, cum uenitis AMst ${ }^{\mathrm{var}} \mathrm{TES}^{\mathrm{var}}$, conuenientibus uobis $\mathrm{AMst}^{\mathrm{D}}$, cum conueneritis PEL $^{\text {var }}$ TES $^{\text {var }}$, cum ueneritis PEL ${ }^{\text {var }}$, cum conuenietis TES ${ }^{\text {var }}$

ad manducandum inuicem

expectate $\left(5^{8}\right) 7^{6}$ ] expectantes $\mathrm{TES}^{\mathrm{var}}$

11:34 Present: $5^{1} 545^{8} 61757677788889262$ AMst PEL ${ }^{A}$ TES SPE

si quis ] si qui TES ${ }^{\text {var }}$, qui TES ${ }^{\text {var }}$ + autem 89 TES $^{\text {var }}$

esurit 61 ] esuriuit TES ${ }^{\mathrm{var}}$, esurierit TES ${ }^{\mathrm{var}}$

domi ] in domo uel domi 77, domui Tes var

manducet ut non ] manducet et non AMst ${ }^{\text {var }}$ TES $^{\text {var }}$

in AMst $^{\text {var }}$ ] ad 6175767789 AMsted PEL $^{\text {var }}$ TES $^{\text {var }}$

iudicium ] iudicio AMst ${ }^{\mathrm{var}} \mathrm{SPE}^{\mathrm{var}}$

conueniatis ] conuenitis 262 , ueniatis TES ${ }^{\text {var }}$

cetera autem 61 ] abs. TES

cum uenero $P E L^{\text {var }}$ ] cum uenerim $P E L^{\mathrm{A}}$, abs. TES

disponam ] uobis disponam $54^{\mathrm{C}}$, ordinabo 75767789 , disponam uobis 262 , abs. TES

12:1 Present: 515458617375767778848889 AMst $^{\dagger}$ PEL $^{\mathrm{A}}$

de spiritalibus 51 (88) ] fratres de spiritalibus 73 , abs. 84

$\dagger \quad$ 1Cor. 12:1. Vogels' edition of AMst erroneously has uolo for nolo in this verse. 
autem ] om. 73 77, abs. 84; + fratres $84 \mathrm{AMst}^{\mathrm{AD}}$

nolo uos ignorare

fratres ] om. $7384 \mathrm{AMst}^{\mathrm{AD}}$

12:2 Present: $5^{1} 545^{8} 6173757677788488$ 89 AMst PEL A $^{\mathrm{A}}$

scitis ] nescitis PEL var; + autem PELA, + enim PEL var

quoniam AMst ${ }^{\text {var }}$ ] quia $5^{1} \mathrm{AMst}^{\text {ed }}$

cum gentes essetis $\mathrm{AMst}^{\mathrm{var}} \mathrm{PEL}^{\mathrm{var}}$ ] gentes eratis $61757689 \mathrm{AMst}^{\text {ed }} \mathrm{PEL}^{\mathrm{A}}$, cum gentes eratis 77 , gentes $\langle a b s\rangle$.

ad simulacra muta $\left.51\left(54^{*}\right) \mathrm{PEL}^{\mathrm{var}}\right]$ simulacrorum formae similes $61 P E L^{A}$, idolorum sine uoce 7576 , ad simulacrorum formationes $77, a b s$. 84 , idolorum formae 89, simulacrorum forma $\mathrm{AMst}^{\mathrm{ed}}$, ad simulacrorum formam $\mathrm{AMst}^{\mathrm{var}}$, ad idola muta AMst ${ }^{\mathrm{AD}}$, ad simulacra PEL var; + euntes 61757689 AMst $^{\text {ed }}$ PEL var

prout ducebamini ] prout decebamini 75, abs. 84, quam praecipites ferebamini $\mathrm{AMst}^{\mathrm{AD}}$

euntes ] om. 61757689 AMst PEL ${ }^{\text {var }}$, abs. 84

12:3 Present: 5154586173757677788889 AMst PEL $^{\mathrm{A}} \mathrm{SPM}^{\dagger}$

ideo SPM ${ }^{\text {var }}$ ] propter quod $7576 \mathrm{AMst} \mathrm{SPM}^{\text {ed }}$, ideoque 89 ; + uel propter quod 77

notum uobis facio ] notesco uobis 77 , uobis facio notum $78^{*}$, facio uobis notum $78^{\mathrm{C}}$, notum facio uobis $\mathrm{AMs} \mathrm{t}^{\mathrm{AD}}$

quod $\mathrm{AMst}^{\mathrm{AD}} \mathrm{SPM}^{\mathrm{ed} 2}$ ] quia $75767789 \mathrm{AMst}^{\mathrm{ed}} \mathrm{SPM}^{\mathrm{ed}}$

nemo in spiritu dei ] nemo in spiritu $\mathrm{SPM}^{\mathrm{var}}$

loquens $\left(73^{*}\right)$ AMst $^{\text {var }}$ SPM $^{\text {var }}$ ] om. 61757677 AMst $^{\text {ed }}$ PEL $^{\text {var }}$ SPM $^{\text {ed }}$

$\operatorname{dicit}\left(73^{*}\right)$ ] dicat $\mathrm{AMst}^{\mathrm{AD}}$ *

anathema iesu $5473 \mathrm{AMst}^{\mathrm{var}}$ ] anathema iesum $61757677 \mathrm{AMst}^{\mathrm{ed}} \mathrm{SPM}^{\mathrm{var}}$

et nemo potest dicere $(75)$

dominus iesus SPM ${ }^{\mathrm{var}}$ ] dominum iesum 6175767789 AMst PEL $^{\mathrm{A}}$ SPM $^{\mathrm{ed}}$, christum dominum iesum $\mathrm{SPM}^{\mathrm{ed} 2}$

nisi in spiritu sancto

12:4 Present: 5154586173757677788889 AMst PELA CAr SPM

diuisiones 6175

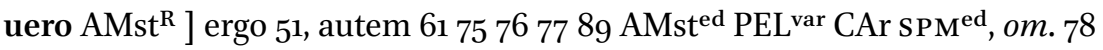
SPM ${ }^{\text {var }}$

$\dagger{ }_{1}$ Cor. 12:3-6. These verses are quoted twice in SPM. 
gratiarum sunt 58 ] donationum sunt $7576 \mathrm{AMst}^{\mathrm{AD}} \mathrm{SPM}$, donationes sunt

89 ; + uel donationum sunt 77

idem autem ] idem uero AMst ${ }^{\mathrm{AD}} \mathrm{SPM}$, om. CAr; + et 61

spiritus ] dominus $88^{*}$

12:5 Present: $51545^{8} 6173757677788889$ AMst PEL ${ }^{A}$ SPM

et diuisiones 6175 ] om. $88^{*}$

ministrationum sunt $\left.78 \mathrm{AMst}^{\mathrm{var}} \mathrm{SPM}^{\mathrm{var}}\right]$ om. $7388^{*}$, ministeriorum sunt 75 7677 AMst $^{\text {ed }}$ spM $^{\text {ed }}$, sunt 89

idem autem $\mathrm{AMst} \mathrm{AD}^{\mathrm{AD}}$ ] om. $7388^{*}$, idem uero $7576 \mathrm{AMst}^{\mathrm{ed}} \mathrm{SPM}$, et idem 77 dominus ] om. $7388^{*}$, deus $75 \mathrm{AMst}^{\mathrm{var}}$, christus $\mathrm{AMst}{ }^{\mathrm{AD}}$

12:6 Present: $5^{1} 545^{8} 6173757677788889$ AMst PEL $^{\mathrm{A}}$ SPM

et diuisiones 61 ] om. 73

operationum sunt 58 ] ministeriorum sunt 89

idem uero $\mathrm{SPM}^{\mathrm{var}}$ ] idem autem 5478 AMst PEL ${ }^{\mathrm{var}}$, sed idem $\mathrm{SPM}^{\text {ed }}$

deus $\mathrm{SPM}^{\mathrm{var}}$ ] dominus $73 \mathrm{SPM}^{\mathrm{ed}}$

qui operatur omnia in omnibus $\left(88^{*}\right)$

12:7 Present: $5^{1} 545^{8} 6173757677788889$ AMst PEL ${ }^{A}$ SPM

unicuique

autem ] om. AMst ${ }^{\text {var }}$, quidem SPM ${ }^{\text {var }}$ + nostrum 89 PEL $^{\mathrm{A}}$ SPM

datur ] data est 89; + autem AMstvar

manifestatio spiritus $545^{8}$

ad utilitatem ] lac. $76^{*}$, om. PELvar

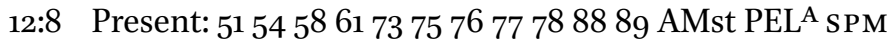

alii quidem ] alii autem $61 \mathrm{AMst}^{\mathrm{AD}} \mathrm{PEL}^{\text {var(ed) }} \mathrm{SPM}$, alii enim $757^{\mathrm{C}} 89$, et alii quidem 78 , cuidam enim AMst ${ }^{\text {ed }}$, lac. $76^{*}$; + nobis PEL ${ }^{\text {var }}$

per spiritum datur ] datur per spiritum 6189 AMst PEL ${ }^{\text {var(ed) }}$ SPM, per spiritum datur per spiritum 73 , lac. $76^{*}$

sermo sapientiae $51545^{8} 7^{6} 7788$ ] sapientiae sermo $61 \mathrm{PEL}^{\mathrm{var}}$, sapientiae

AMst ${ }^{\text {var }}$, sermo scientiae SPM ${ }^{\text {var }}$, lac. $76^{*}$; + ad utilitatem PEL var

alii autem ] alii $757689 \mathrm{AMst}^{\mathrm{var}} \mathrm{PEL}^{\mathrm{var}} \mathrm{SPM}^{\mathrm{var}}$, alii uero $\mathrm{AMst}^{\mathrm{ed}} \mathrm{SPM}^{\mathrm{ed}}$

sermo scientiae $51545^{8} 76$ PEL $\left.^{\text {var(ed) }}\right]$ scientiae PEL ${ }^{A}$, sermo sapientiae $\mathrm{SPM}^{\mathrm{var}}$

secundum eundem spiritum

12:9 Present: $51545^{8} 6173757677788889$ AMst PEL ${ }^{A}$ SPM

alteri ] alii 75767789 AMst PEL var SPM 
fides $\mathrm{AMst}^{\mathrm{AD}}$ ] gratia curationum $\mathrm{AMst}^{\mathrm{ed}}$ in eodem ] in uno AMst spiritu ] spiritum 73

alii gratia $58 \mathrm{AMst}^{\mathrm{AD}} \mathrm{SPM}^{\mathrm{var}}$ ] alii dona uel gratiae $77^{*}$, alii dona uel gratia $77^{\mathrm{C}}$, alii gratiae $7889 \mathrm{PEL}^{\text {var }} \mathrm{SPM}^{\text {ed }}$, alii $\mathrm{AMst}^{\text {ed }}$

sanitatum $78 \mathrm{AMst}^{\mathrm{AD}} \mathrm{PEL}^{\mathrm{A} *}$ ] curationum $61 \mathrm{PEL}^{\text {var }} \mathrm{SPM}^{\text {var }}$, sanitatem 75 , sanitatum curationum $7^{8 \mathrm{gl}}$, fides AMsted, sanitatium PEL ${ }^{\mathrm{AC}} \mathrm{SPM}^{\mathrm{ed}}$ in uno spiritu $(75) 78$ ] in eodem spiritu $777^{\mathrm{gl}} \mathrm{AMst}$

12:10 Present: 51545861737576777888 89 AMst PELA SPM alii operatio 5873 ] alii operationis AMstrar uirtutum $78 \mathrm{SPM}^{\mathrm{var}}$ ] uirtutis $757^{6} 777^{\mathrm{gl}} 89 \mathrm{SPM}^{\mathrm{ed}}$ alii prophetatio $\mathrm{AMst}{ }^{\mathrm{AD}}$ ] alii prophetia $5^{1}{ }_{54}{ }^{\mathrm{C}} 586175767788 A M s^{\text {ed }} \mathrm{PEL}^{\text {var }}$ $\mathrm{SPM}^{\mathrm{var}}$, alii prophe[3] $54^{*}$

alii discretio $5861 \mathrm{SPM}^{\mathrm{var}}$ ] alii separatio $757689 \mathrm{SPM}^{\mathrm{ed}}$ spirituum (78) SPM ${ }^{\text {var }}$ ] spiritum 6173 , spiritus $\mathrm{SPM}^{\mathrm{ed}}$ alii genera linguarum $\mathrm{AMst}^{\mathrm{AD}}$ ] alii interpretatio sermonum $\mathrm{AMst}^{\mathrm{var}}$, om. AMsted alii ] + autem 77 interpretatio sermonum 5878 (88) $\mathrm{AMst}^{\mathrm{AD}} \mathrm{SPM}^{\mathrm{var}}$ ] interpretatio linguarum $7576777^{\mathrm{gl}} 89 \mathrm{AMst}^{\mathrm{ed}}$, genera linguarum $\mathrm{AMst}^{\mathrm{var}}$, interpretationes linguarum $\mathrm{SPM}^{\mathrm{ed}}$

12:11 Present: 5154586173757677788889 AMst PEL $5^{\mathrm{A}}$ SPM

haec autem omnia 51545876 (77) ] omnia autem haec $\mathrm{AMst}^{\mathrm{ed}} \mathrm{SPM}^{\mathrm{ed}}$, omnia haec AMstvar SPM ${ }^{\text {var }}$

operatur 73 ] operantur AMst ${ }^{\text {var }}$

unus atque idem spiritus diuidens 7375

singulis $78 \mathrm{SPM}^{\text {var }}$ ] unicuique $7576 \mathrm{AMst}^{\mathrm{ed}} \mathrm{SPM}^{\mathrm{ed}}$, unicuique uel singulis 77 , unicuique $7^{\mathrm{gl}}$, se unicuique 89 , om. $\mathrm{AMst}^{\mathrm{AD}}$, propria $\mathrm{SPM}^{\mathrm{var}}$ prout uult

12:12 Present: 51545861737576777888 89 AMst PEL ${ }^{\mathrm{A}}$

sicut enim ] sicut autem 61 PEL $^{\text {var }}$

corpus unum est

et membra AMst ${ }^{\mathrm{var}}$ PEL $\left.{ }^{\mathrm{var}}\right]$ membra autem $75767789 \mathrm{AMst}^{\text {ed }} \mathrm{PEL}^{\mathrm{A}}$

habet multa ] multa habet 51, [4] multa $54^{*}$

omnia autem membra 78 ] omnia membra 7378

corporis $\mathrm{AMst}^{\mathrm{AD}}$ ] de corpore $61 \mathrm{PEL}^{\mathrm{var}}$, de corpore uno $757689 \mathrm{PEL}^{\mathrm{var}(e d)}$, ex uno corpore AMsted 
cum sint multa unum ] multa cum sint unum $\mathrm{AMst}^{\mathrm{AD}}$ corpus sunt ] est corpus $75767789 \mathrm{AMst}^{\mathrm{AD}}$, cor[4-6] 88*, sunt corpus AMsted

ita et christus ] ita et spiritus 73

12:13 Present: $5^{1} 545^{8} 6173757677788889$ AMst PEL ${ }^{\mathrm{A}}$

etenim ] nam et 7576 , nam 89

in ] om. 757689 PEL var

uno spiritu ] spiritu uno $61 \mathrm{PEL}^{\mathrm{var}}$, spiritu 77 89, om. PEL ${ }^{\text {var }}$

omnes nos ] nos omnes $75767789 \mathrm{AMst}^{\text {ed }} \mathrm{PEL}^{\mathrm{var}(\mathrm{ed})}$, omnes AMst ${ }^{\mathrm{var}}$

in unum corpus ] in uno spiritu PEL var

baptizati sumus 5473 ] sumus baptizati 61 PEL var

siue iudaei siue 51545861737678

gentiles 78 ] serui 54 , graeci $617576777^{g l} 89$ AMst PEL var(ed)

siue 73 ] om. AMst ${ }^{\mathrm{var}}$

serui 73 ] gentiles 54, liberi 61, om. AMstvar, libera PEL var(ed)

siue liberi 73 ] siue serui 61 PEL $^{\text {var(ed) }}$

et ] om. PEL ${ }^{\text {var }}$ + enim 73

omnes ] + in $54^{\mathrm{C}} 78$

unum spiritum PEL var(ed) $]$ uno spiritu $5^{1} 54^{\mathrm{C}} 58737888^{\mathrm{C}} \mathrm{PEL}^{\mathrm{A}}$

potati sumus ] potauimus $617589 \mathrm{AMst}^{\text {ed }} \mathrm{PEL}^{\text {var(ed) }}$, potauimus uel potabimus uel potauimur 77 , optauimus $\mathrm{AMst}^{\mathrm{var}}$, potamus $\mathrm{AMst} \mathrm{AD}^{\mathrm{AD}}$

\pm per iesum christum dominum nostrum 73 , et omnes uno spiritu potati sumus PEL var

12:14 Present: 51545861757677788889 AMst PEL ${ }^{A}$

nam et ] etenim $75767789 \mathrm{AMst}^{\mathrm{AD}}$

corpus non est unum membrum sed multa 88

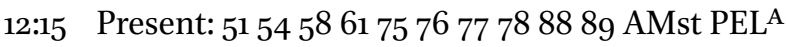

si dixerit pes

quoniam non sum manus ] quia non sum manus 89 AMst

non sum de corpore ] non sum ex corpore 757689 AMst $^{\mathrm{AD}}$

non ] et $\mathrm{AMst}^{\mathrm{var}}$, num $\mathrm{AMst}^{\mathrm{R}}$

ideo non est ] propterea non est $75767789 \mathrm{AMst}^{\mathrm{ed}}$, ideo propterea non est AMst ${ }^{\text {var }}$

de corpore ] ex corpore $757689 \mathrm{AMst}^{\mathrm{AD}}$, de uel ex corpore 77 
12:16 Present: $51545^{8} 61757677788889$ AMst PEL $^{\mathrm{A} \dagger}$

et si dixerit ] si dixerit $7576 \mathrm{AMst}^{\mathrm{ed}}$, et dixerit $\mathrm{AMst}^{\mathrm{var}}$

auris ] auricula 757689 AMst PEL $^{\text {var }}$, auricula uel auris 77

quia ] quoniam $61 \mathrm{PEL}^{\mathrm{var}}$, om. PEL ${ }^{\mathrm{var}}$

non sum oculus 54

non sum de corpore ] om. 51, non sum ex corpore 75767789 AMst $^{\mathrm{AD}}$ PEL $^{\text {var }}$ non ] num $\mathrm{AMst}^{\mathrm{R}}$

ideo $78 \mathrm{AMst}^{\mathrm{AD}}$ ] propterea $7^{\mathrm{gl}} \mathrm{AMst} \mathrm{t}^{\mathrm{ed}}$

non est de corpore ] non est ex corpore $75767789 \mathrm{AMst}^{\mathrm{AD}}$

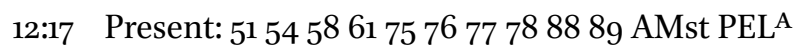

si totum corpus oculus 54 ] si totum corpus est oculus PEL var

ubi auditus ] ubi est auditus $61 \mathrm{AMst}^{\mathrm{var}} \mathrm{PEL} \mathrm{L}^{\mathrm{var}}$

si totum $\left.\left(\mathrm{PEL}^{\mathrm{A} *}\right)^{\mathrm{C}}\right]$ si totus $5^{1} 54$

auditus ] auditur AMst ${ }^{\text {var }}$

ubi odoratus] ubi adoratus 54, ubi est odoratus $61 \mathrm{AMst}^{\mathrm{var}}$

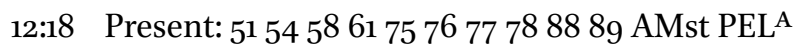

nunc autem ] non hunc autem PEL var

posuit deus membra $88 \mathrm{AMst}^{\text {var }}$ ] deus posuit membra $757677 \mathrm{AMst}^{\mathrm{ed}}$

unumquodque eorum in corpore $\langle 51\rangle 88$ ] in corpore unumquodque eorum

58 , singula quoque eorum in corpore 7576 , singulorum in corpore 89 ,

unumquodque in corpore AMst ${ }^{\mathrm{var}}$, singula eorum corpori $\mathrm{AMst}^{\mathrm{AD}}$

sicut uoluit ] prout uoluit 757689 AMst $^{\mathrm{AD}}$ PEL $^{\text {var }}$

12:19 Present: $5^{1} 545^{8617576777888 ~ 89}$ AMst PEL $^{\mathrm{A}}$

quod si essent $54 \mathrm{AMst}^{\text {var }}$ ] si autem fuissent $\mathrm{AMst}^{\mathrm{ed}}$

omnia unum membrum ubi corpus 7588

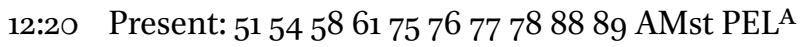

nunc autem ] nunc uero AMst ${ }^{\text {var }}$

multa quidem membra 88 ] multa membra 61757689 PEL var $^{\text {va }}$

unum autem corpus ] sed unum corpus 757689

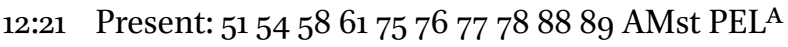

non potest ] + autem $5^{1} 5^{8} 757688 \mathrm{AMst}^{\text {ed }} \mathrm{PEL}^{\mathrm{A}}$

dicere oculus manui $54 \mathrm{AMst}^{\text {var }}$ ] oculus dicere manui $5^{8} 75767778 \mathrm{AMst}^{\text {ed }}$

$\dagger \quad 1$ Cor. 12:16. In the Pseudo-Jerome tradition of PEL, this verse and its exegesis appear after 1Cor. 7:21. 
opera tua non indigeo 78 ] opere tua non indigeo 54 , opera tua non desidero $61 \mathrm{PEL}^{\text {var(ed) }}$, non est mihi necessaria 7576 , necessitatem tuam uel tui non habeo 77 , necessitatem tui non habeo $7^{\mathrm{g}}$, non es mihi necessaria 89 $\mathrm{AMst}^{\mathrm{AD}}$, opera tua non egeo $\mathrm{AMst}^{\mathrm{var}}$, operam tuam non desidero PEL $\mathrm{Par}^{\mathrm{var}}$ aut iterum caput pedibus 89 ] aut iterum caput dicere pedibus AMstvar non estis mihi necessarii 515478 ] non desidero operam uestram 7576 89, necessarium uos non habeo 77 , non estis necessarium uos $78^{\mathrm{gl}}$, non desidero opera uestra $\mathrm{AMst}^{\mathrm{AD}}$, non estis mihi necessarium $\mathrm{AMst}^{\mathrm{var}}$, nec estis mihi necessarii PEL var

12:22 Present: $51545^{8617576} 77788889$ AMst PEL ${ }^{\mathrm{A}}$

sed multo magis ] sed magis 89

quae uidentur membra 51545861767888 ] quae putantur membra $\mathrm{AMst}^{\mathrm{AD}}$ corporis infirmiora esse ] corpus infirmiora esse $54^{*}$

necessariora sunt ] necessaria sunt $75767788^{*} 89 \mathrm{AMst}^{\mathrm{var}}$, magis necessaria sunt $\mathrm{AMst}^{\mathrm{D}}$

12:23 Present: 51545861757677788889 AMst PEL ${ }^{\mathrm{A}}$

et quae putamus 515458617688

ignobiliora membra esse corporis 88 ] ignobiliora esse membra corporis $515861757677 \mathrm{AMst}^{\mathrm{ed}}$, ignobiliora membra corporis esse 54, inferiora membra corporis esse $\mathrm{AMst}^{\mathrm{var}}$, inhonestiora esse corporis $\mathrm{AMst}^{\mathrm{AD}}$, membra corporis ignobiliora esse PEL var

his honorem abundantiorem circumdamus 54586188 AMst $^{\mathrm{AD}}$ ] honorem his abundantiorem circumdamus 51 , his honorem abundantiorem circumdamur 77 , his abundantiorem honorem circumdamus $A M s t^{e d}$, his maiorem honorem circumdamur AMst ${ }^{\mathrm{var}}$, his abundantiorem circumdamus honorem PEL var

et quae $51545^{8} 61767888^{\circ}$ ] et $\mathrm{q}\left[\mathrm{o}^{-1}\right] 88^{*}$, et abundantiora et 89 , et $\mathrm{AMst}{ }^{\mathrm{AD}}$ inhonesta sunt ] uerecundiora $757689 \mathrm{AMst}^{\mathrm{AD}}$, inhonesta uel turpia 77 nostra ] uestra $89^{*}$, nobis AMst ${ }^{\mathrm{var}}$; + sunt 77

abundantiorem honestatem $54\left(7^{*}\right) 88$ ] abundantiorem honorem 51 $A M s^{e d}$, abundantiorem 58 , honestatem abundantiorem $61757^{6} 77$, reuerentiam abundantiorem $\mathrm{AMst}^{\mathrm{var}}$, maiorem honestatem $\mathrm{AMst}^{\mathrm{AD}}$

habent

12:24 Present: 51545861757677788889 AMst PELA SPE

honesta autem $\mathrm{AMst}^{\mathrm{var}}$ ] quae autem honesta sunt $61 \mathrm{AMst}^{\mathrm{ed}} \mathrm{PEL}{ }^{\mathrm{var}(e d)}$, honestiora autem 7576 , quae autem sunt honesta $\mathrm{AMst}^{\mathrm{AD}}$, et quae honesta $\mathrm{AMst}^{\mathrm{var}}$, abs. SPE 
nostra ] abs. SPE; + membra 58

nullius egent ] ullius egent 58 , nulli egent $\mathrm{AMst}^{\mathrm{var}}$, non indigent $\mathrm{AMst}^{\mathrm{AD}}$, abs. SPE; + honorem 7576

sed deus temperauit corpus $\left.\left(5^{*}\right)\right]$ sed temperauit corpus PEL ${ }^{A *}$

ei ] si 75 76, eius AMstvar, om. AMst ${ }^{\mathrm{AD}} \mathrm{PEL}^{\text {var }}$

cui deerat $\mathrm{AMst}^{\mathrm{var}}$ ] cui deest $61 \mathrm{AMst}^{\mathrm{ed}} \mathrm{PEL}^{\mathrm{var}(\mathrm{ed})}$

abundantiorem $5154586188 A M$ sted $^{\text {ed }}$ ] plus $75767789 \mathrm{AMst}^{\mathrm{AD}}$, ampliorem $\mathrm{AMst}^{\mathrm{var}}$

tribuendo honorem ] tribuendo uel abundantiorem dando honorem 77, retribuendo honorem $\mathrm{AMst}^{\mathrm{var}}$, honorem tribuendo $\mathrm{AMst}^{\mathrm{var}}$, honestatem tribuendo et honorem PEL var

12:25 Present: 51545861757677788889 AMst PEL $5^{\mathrm{A}}$ SPE

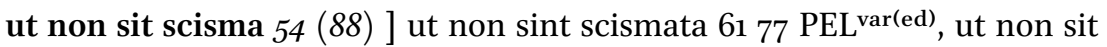
scissura 757689 , ut non sit separatio AMst $\mathrm{AD}^{\mathrm{AD}}$

in corpore sed $\left.\left(78^{*}\right)\right]+$ in $5^{1} 5^{861} 77^{88^{C}}$ AMsted $^{\text {PEL }}{ }^{\text {var }}$ SPE, + ut 54

id ipsum ] eadem $\mathrm{AMst}^{\mathrm{AD}}$, ipsa AMst ${ }^{\text {var }}$; + sint 77

pro inuicem ] inuicem pro se $\mathrm{AMst}{ }^{\mathrm{AD}}$

sollicita sint membra $5^{8}$ ] sollicita sunt membra 6178 , cogitent membra $\mathrm{AMst}^{\mathrm{AD}}$

12:26 Present: 51545861757677788889 AMst PEL $5^{\mathrm{A}}$ SPE

et si quid ] siue PEL ${ }^{\text {var }}$

patitur unum membrum ] patimur unum membrum $61^{*}$

conpatiuntur omnia membra 587778 ] conpatiantur omnia membra 7576

siue gloriatur $\mathrm{AMst}^{\mathrm{var}} \mathrm{PEL}^{\mathrm{var}} \mathrm{SPE}^{\mathrm{var}}$ ] siue glorificatur $54^{*} 6189 \mathrm{AMst}^{\mathrm{ed}} \mathrm{PEL}^{\mathrm{A}}$

$\mathrm{SPE}^{\text {ed }}$, siue glorificetur 7576 , siue inlustratur $\mathrm{AMst}^{\mathrm{AD}}$

unum membrum

congaudent omnia membra $(76)$ ] congaudeant omnia membra $\mathrm{AMst}^{\mathrm{R}}$

12:27 Present: 515458617576777888 89 AMst PEL ${ }^{A}$ SPE

uos autem ] uos 89

estis corpus christi ] corpus estis christi 77

et membra ] et membrum AMst $\mathrm{t}^{\mathrm{var}}$

de membro ] om. $61 \mathrm{PEL}^{\mathrm{var}}$, ex membro 75 76, ex parte $7789 \mathrm{AMst}^{\mathrm{AD}}$

12:28 Present: 51545861757677788889 AMst PELA ${ }^{A}$ SP

et quosdam quidem ] et quosdam $7576 \mathrm{AMst}^{\mathrm{R}}$

posuit deus ] posuit $S E^{\text {var }}$

in ecclesia ${ }_{51} 61$ ] in ecclesiam AMstrar PEL ${ }^{\text {var }}$ 
primum apostolos ] primo apostolos $\mathrm{AMst}^{\mathrm{AD}}$, primum quidem apostolos AMstvar

secundo prophetas 515861 ] secundum prophetas $757788^{*}$ PEL $^{\text {var }}$

tertio doctores ] tertio magistros $757689 \mathrm{AMst}^{\mathrm{AD}}$, tertio magistros uel doctores 77

deinde uirtutes

exin PEL var ] exinde $5^{1} 5^{\mathrm{C}} 5^{8} 78 \mathrm{AMst}^{\mathrm{var}} \mathrm{SPE}^{\mathrm{var}}$, om. $75767789 \mathrm{AMst}^{\mathrm{var}} \mathrm{PEL}^{\mathrm{A}}$, deinde AMsted

gratias curationum $58 \mathrm{AMst}^{\mathrm{var}} \mathrm{PEL}^{\mathrm{var}}$ ] gratia curationum $54 * \mathrm{SPE}^{\mathrm{ed}}$, donationes sanitatium 75 , donationes sanitatum 76 , donationes uel gratias sanitatum 77, sanitatum donationes 89, gratiam curationum AMsted $\mathrm{SPE}^{\mathrm{var}}$, gratia $\mathrm{AMst}^{\mathrm{var}}$, donationum sanitates $\mathrm{AMst}^{\mathrm{AD}}$, om. $\mathrm{PEL}^{\mathrm{A}}$

opitulationes 5888 ] adiutoria 757689 , om. AMst ${ }^{\mathrm{AD}}$, gubernationes opitulationes SPEvar; + uel adiutoria 77

gubernationes $\left.5^{8}\left\langle{ }_{76}\right\rangle \mathrm{SPE}^{\mathrm{var}}\right]$ om. AMst ${ }^{\mathrm{var}} \mathrm{SPE}^{\mathrm{ed}} ;+$ et $\mathrm{AMst}^{\mathrm{var}}$

genera linguarum $\left.P E L^{\text {var }}\right]$ om. $\mathrm{PEL}^{\mathrm{A}}$

\pm interpretationes sermonum $58 \mathrm{AMst}^{\mathrm{ed}} \mathrm{SPE}^{\mathrm{var}}$, dona 89 , interpretatio sermonum AMst ${ }^{\text {var }}$

12:29 Present: $5^{1} 545^{8} 617576777888$ 89 AMst PELA SPE

numquid omnes apostoli 77 ] $\mathrm{om} .54^{*}$

numquid 77 ] lac. $78^{*}$

omnes prophetae numquid 5154586176777888

omnes doctores ] omnes magistri $757689 \mathrm{AMst}^{\mathrm{AD}}$, magistri 77

12:30 Present: $51545^{8} 61757677788889$ AMst PEL ${ }^{\text {var† }}$ SPE

numquid omnes uirtutes $54 * 77$ ] num omnes uirtutes $\mathrm{AMst}^{\mathrm{R}}$

numquid omnes 54 * $77 \mathrm{AMst}^{\mathrm{var}}$ ] num omnes AMst $\mathrm{Ad}^{\mathrm{ed}}$

gratiam $5^{8}$ ] donationes 757689 , dona uel gratias 77 , gratiarum AMst ${ }^{\text {var }}$, donationem $\mathrm{AMst}^{\mathrm{AD}}$

habent curationum 58 ] habent sanitatium 75 89, habent sanitatum 76 $\mathrm{AMst}^{\mathrm{AD}}$, habent sanitatum uel curationum 77

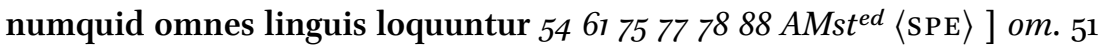
AMstvar

numquid omnes (54) 77 ] num omnes 88

interpretantur 89 ] interpetrantur 75

$\dagger{ }_{1}$ Cor. 12:30. The absence of this verse from PELA may be deliberate, because the previous verse ends et reliqua. 
12:31 Present: 51545861757677788889 AMst PEL $^{\text {A }}$ SPM SPE

aemulamini autem $515861 \mathrm{SPM}^{\text {var }}$ ] aemulamini $\mathrm{SPM}^{\mathrm{ed}}$

charismata $5158617888 \mathrm{AMst}^{\text {ed }} \mathrm{SPM}^{\mathrm{var}}$ ] dona $75767789 \mathrm{AMst}^{\mathrm{AD}} \mathrm{SPM}^{\mathrm{ed}}$

maiora SPM $^{\text {var }}$ ] meliora $5^{1} 545^{8} 617576778889$ AMst PEL $^{\text {var(ed) }}$ SPM $^{\text {ed }}$ $S P E^{\text {var }}$

et ] om. 75767789 AMst $^{\text {var }}$

adhuc ] + magis AMst ${ }^{\text {ed }}$

excellentiorem $\left.58 \mathrm{sPM}^{\mathrm{var}}\right]$ maiorem $757689 \mathrm{sPM}^{\mathrm{ed}}$

uiam uobis demonstro $\left.\left(88^{*}\right) \mathrm{AMst}^{\mathrm{var}} \mathrm{SPM}^{\mathrm{var}}\right]$ uiam uobis demonstrabo

61 PEL ${ }^{\text {var }}$, uiam demonstro uobis 77 , uiam nobis demonstro 89, uobis uiam demonstro AMst ${ }^{\text {ed }} \mathrm{PEL}^{\mathrm{A}} \mathrm{SPM}^{\text {ed }} \mathrm{SPE}^{\mathrm{ed}}$, uobis uiam demonstrabo $S P E^{\mathrm{var}}$

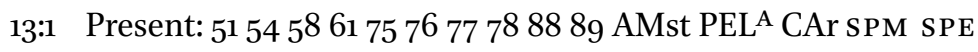

si linguis hominum loquar ] si linguis loquar $\mathrm{SPM}^{\mathrm{var}}$

et angelorum $61 \mathrm{SPM}^{\text {var }}$ ] aut angelorum $\mathrm{SPM}^{\mathrm{ed}}$

caritatem autem non habeam ] caritatem non habeam AMst ${ }^{\mathrm{var}}$, abs. CAr

factus sum 78 AMst $\left.^{\text {var }} \mathrm{SPM}^{\mathrm{var}}\right]$ in unum sum 7576 , unum sum $777^{\mathrm{gl}} 89$

AMst ${ }^{\text {ed }}$ PEL ${ }^{\text {var(ed) }}$ SPM $^{\text {ed }}$, abs. CAr

uelut 58 ] ut $7576 \mathrm{AMst}^{\mathrm{AD}}$ sPM, aut 89, abs. CAr

aes $51545877 \mathrm{PEL}^{\mathrm{var}} \mathrm{SPE}^{\mathrm{var}}$ ] aeramentum 61757689 AMst $P E L^{A} \mathrm{SPM}^{\mathrm{SP}} \mathrm{E}^{\mathrm{ed}}$, abs. CAr

sonans AMst ${ }^{\text {var }}$ ] resonans AMst ${ }^{\mathrm{ed}}$, abs. CAr

aut cymbalum tinniens 5488 ] abs. CAr

13:2 Present: $5^{1} 545^{8} 61757677788889$ AMst PEL $^{\text {A }}$ TES SPM SPE

et si habuero AMst ${ }^{\mathrm{var}}$ TES $^{\mathrm{var}}$ ] et si habeam 75767789 AMst $^{\text {ed }}$ SPM, abs.

TES ${ }^{\text {ed }}$

prophetiam et 5861 ] abs. TES

nouerim AMst ${ }^{\mathrm{var}}$ ] sciero $\mathrm{AMst} \mathrm{t}^{\mathrm{ed}} \mathrm{SPM}^{\mathrm{var}}$, nouero $\mathrm{AMst}^{\mathrm{A}}, \mathrm{sciam} \mathrm{AMst}^{\mathrm{R}}, a b s$.

TES

mysteria omnia 54586188 AMst $^{\text {var }}$ ] omnia mysteria AMst ${ }^{\text {ed }}$, omnia sacramenta SPM ${ }^{\mathrm{var}}$, abs. TES

et $\mathrm{AMst}^{\text {var }}$ ] et si $58 \mathrm{AMst}^{\text {ed }}$, abs. TES

omnem scientiam $54 \mathrm{AMst}^{\mathrm{var}}$ ] omnem scientiam habuero 58 , habeam omnem scientiam AMst ${ }^{\text {ed }}$, abs. TES

\pm caritatem autem non habeam nihil mihi prodest $\mathrm{AMst}^{\mathrm{ed}}$, et caritatem non habeam nihil mihi prodest $\mathrm{AMst}{ }^{\mathrm{AD}}$, caritatem non habeam nihil mihi prodest AMst ${ }^{\text {var }}$

et ] et si $5^{1} 5461757^{6} 7788^{\mathrm{C}} 89$ AMst PEL TES ${ }^{\text {ed }}$ SPM SPEvar, om. TES $S^{\text {var }}$ 
habuero ] om. $5^{8}$ TES $^{\text {var, }}$, habeam $6175767789 \mathrm{AMst}^{\mathrm{AD}} \mathrm{PEL}^{\mathrm{var}(\mathrm{ed})}$; + in me TES var

omnem fidem ita ut montes transferam (54) ] fidem ita ut montem transferam TES ${ }^{\mathrm{var}}$

caritatem autem non $\mathrm{AMst}^{\mathrm{AD}} \mathrm{TES}^{\mathrm{var}}$ ] om. $\mathrm{AMst}^{\mathrm{ed}}$, agapem autem non TES Sed $^{\text {. }}$

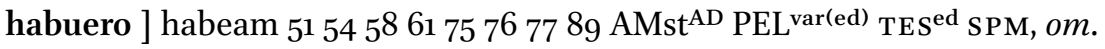
AMst $^{\text {ed }}$, haberem TES ${ }^{\text {var }}$

nihil sum $5154 \mathrm{AMst}^{\mathrm{AD}}$ ] nihil mihi prodest 61 , om. AMst $^{\text {ed }}$, nihil mihi prode est PEL ${ }^{\text {var(ed) }}$, nihil proficio TES ${ }^{\text {var }}$

13:3 Present: $5^{1} 545^{8} 61757^{6} 777^{8} 88$ 89 AMst PEL ${ }^{\mathrm{A}}$ TES SPM SPE

et si $S M^{\text {var }}$ ] et $89^{*}$, si SPM ${ }^{\text {ed }}$

distribuero AMst ${ }^{\mathrm{AD}} \mathrm{SPM}^{\mathrm{var}}$ ] om. $757689 \mathrm{PEL}^{\mathrm{var}}$ TES, erogauero AMst ${ }^{\mathrm{ed}}$ $\mathrm{SPM}^{\text {ed }}$, donauero PEL ${ }^{\text {var }}$

\pm omnes facultates meas $61 \mathrm{PEL}^{\mathrm{A}}$, omnes facultates uel substantias meas 77 , substantiam meam SPM ${ }^{\text {var }}$

in cibos $\mathrm{SPM}^{\mathrm{var}}$ ] om. AMst PEL ${ }^{\mathrm{var}} \mathrm{TES}^{\mathrm{var}} \mathrm{SPM}^{\text {ed }}$, et PEL ${ }^{\mathrm{var}}$, in cibo TES ${ }^{\mathrm{var}}$ pauperum $\mathrm{TES}^{\mathrm{var}} \mathrm{SPM}^{\mathrm{var}}$ ] om. $757689 \mathrm{AMst}^{\mathrm{P}} \mathrm{LL}^{\mathrm{var}} \mathrm{TES}^{\mathrm{ed}} \mathrm{SPM}^{\text {ed }}$

\pm diuisero 757689 , distribuero PEL ${ }^{\text {var }}$ TES

omnes facultates meas $\left.78 \mathrm{PEL}^{\mathrm{var}}\right]$ om. $6177 \mathrm{PEL}^{\mathrm{A}} \mathrm{SPM}^{\mathrm{var}}$, omnem substantiam meam 7576 AMst SPM ${ }^{\text {ed }}$, omnes substantias meas $7^{8 \mathrm{gl}}$, omnem meam 89, omnia mea PEL ${ }^{\text {var }}$ TES; + pauperibus PEL ${ }^{\text {var }}$ TES $^{\text {var }}$ SP M $^{\text {ed }}$

et si tradidero ] et tradidero 757689

corpus meum ut (78) ] corpus meum ita ut $51545^{8} 617888 \mathrm{PEL}^{\mathrm{var}(\mathrm{ed})} \mathrm{SPM}^{\mathrm{var}}$ ardeam ] ardeat $\mathrm{TES}^{\mathrm{var}} \mathrm{SPE}^{\mathrm{var}}$; + igni PEL ${ }^{\mathrm{var}}$

caritatem $\mathrm{AMst}^{\mathrm{AD}} \mathrm{TES}^{\mathrm{var}}$ ] om. AMst $^{\text {ed }}$, agapem $\mathrm{TES}^{\text {ed }}$

autem non $\mathrm{AMst}^{\mathrm{AD}}$ ] non 88 , om. $\mathrm{AMst}^{\mathrm{ed}}$

habuero PEL ${ }^{\text {var }}$ ] habeam 546175767789 AMst $^{\mathrm{AD}}$ PEL $^{\mathrm{A}}$ TES SPM, om. AMsted

nihil mihi prodest $5154 \mathrm{SPM}^{\mathrm{var}}$ ] nihil prodest mihi 7576 , nihil proficio $\mathrm{TES}$ ed, nihil sum TES ${ }^{\text {var }}$, nihil mihi prodeest SPM $^{\text {ed }}$

13:4 Present: $5^{1} 545^{8} 61757677788889$ AMst PEL ${ }^{\mathrm{A}}$ TES SPM SPE caritas TES ${ }^{\mathrm{var}}$ ] agape TES ${ }^{\text {ed }}$

patiens $545888 \mathrm{AMst}^{\mathrm{AD}}$ ] magnanima $\mathrm{AMst} \mathrm{ed}^{\mathrm{ed}} \mathrm{TES}^{\mathrm{ed}} \mathrm{SPM}^{\mathrm{var}}$, magna anima TES $^{\text {var }}$, magna enim TES ${ }^{\text {var }}$

est ] et 89; + agape TES ${ }^{\text {ed }}$ + caritas TES ${ }^{\mathrm{var}} \mathrm{SPM}^{\mathrm{var}}$

benigna est $\mathrm{AMst}^{\mathrm{AD}}$ ] iocunda est $\mathrm{AMst}^{\mathrm{ed}}$, patiens est $\mathrm{TES}^{\mathrm{var}}$

caritas $54 \mathrm{TES}^{\mathrm{var}}$ ] agape $\mathrm{TES}^{\mathrm{ed}}$ 
non aemulatur 51545861767788$]+$ caritas 75767789 TES $^{\text {var }}$ SPM $^{\text {ed }}$, + agape TES ${ }^{\text {ed }}$

non agit perperam $S \mathrm{PM}^{\mathrm{var}}$ ] non egit perperam $54^{*}$, non est perpera 7576 $\mathrm{SPM}^{\mathrm{ed}}$, non perperat uel non perperam agit 77 , perperam non agit 89 , non inflatur $\mathrm{AMst}^{\mathrm{ed}}$, non perperam agit $\mathrm{AMst}^{\mathrm{AD}}$, non agit perpere $\mathrm{TE} \mathrm{S}^{\mathrm{var}}$ non inflatur $\mathrm{AMst}{ }^{\mathrm{AD}}$ ] non perperam agit $\mathrm{AMst}^{\mathrm{e}}$, non agit perperam AMst $^{\text {var }}$ + non dehonestatur TES ${ }^{\text {var }}$

13:5 Present: 51545861757677788889 AMst PEL ${ }^{A}$ TES SPM SPE non est ambitiosa $515888 \mathrm{AMst}^{\mathrm{var}} \mathrm{TES}^{\mathrm{var}}$ ] non ambitiosa est $757689 \mathrm{AMst}^{\text {ed }}$

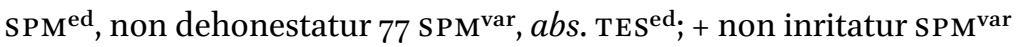
non quaerit quae sua sunt $\left.5154^{c} 5876777888 \mathrm{TES}^{\mathrm{var}}\right]$ non quaerit quae sunt sua $54^{*}$, non quaerit sua uel propria $77, a b s$. TES $S^{\text {ed }}$ non inritatur 5154587677788889 ] om. TES ${ }^{\mathrm{var}} \mathrm{SPM}^{\mathrm{var}}$ non cogitat malum $\left.\left(88^{*}\right)\right]$ om. $\mathrm{SPM}^{\mathrm{var}}$

13:6 Present: $5^{1} 545^{8} 61757677788889$ AMst PEL $^{\mathrm{A}}$ TES SPM SPE non gaudet

super $\left.\mathrm{SPM}^{\mathrm{var}}\right]$ in $\mathrm{AMst}^{\mathrm{ed}}$, om. AMst ${ }^{\mathrm{var}} \mathrm{SPM}^{\mathrm{ed}}$

iniquitatem SPM ${ }^{\mathrm{var}}$ ] iniquitate $5^{8} 7778 \mathrm{AMst}_{\mathrm{SPM}} \mathrm{M}^{\mathrm{ed}}$, iniustitiam TES congaudet autem $54 \mathrm{SPM}^{\text {var }}$ ] conlaetatur autem TES, gaudet autem SPM ${ }^{\text {ed }}$ ueritati $\mathrm{TES}^{\mathrm{var}} \mathrm{SPM}^{\mathrm{var}}$ ] in ueritate $\mathrm{TES} \mathrm{S}^{\mathrm{ed}}$, in ueritatem $\mathrm{TES}^{\mathrm{var}}$, ueritate $S \mathrm{PM}^{\mathrm{ed}}$

13:7 Present: $5^{1} 545^{8} 61757677788889$ AMst PEL $^{\text {A }}$ TES SPM SPE

omnia suffert $54 \mathrm{TES}^{\mathrm{var}}$ ] omnia sufferit 75 , omnia tolerat AMst, omnia diligit TES ${ }^{\text {ed }}$ om. SPM ${ }^{\text {var }}$ + omnia sperat SPM ${ }^{\text {ed }}$

omnia credit omnia sperat $S \mathrm{PM}^{\mathrm{var}}$ ] omnia credit $\mathrm{AMst}^{\mathrm{var}} \mathrm{SPM}^{\mathrm{ed}}$ omnia sustinet $75 \mathrm{AMst}^{\mathrm{AD}}$ ] omnia suffert $\mathrm{AMst}^{\mathrm{ed}}$ \pm omnia diligit $61 P E L^{\text {var }}$, omnia sperat $\mathrm{AMst}^{\mathrm{var}}$

13:8 Present: $5^{1} 545^{8} 61757677788889$ AMst PEL $^{\text {A }}$ TES SPM SPE caritas numquam $77\left(89^{*}\right)$ TES $^{\text {var }}$ ] agape numquam TES $S^{\text {ed }}$ excidit $88^{\mathrm{C}} \mathrm{SPE}^{\mathrm{var}}$ ] excidet $5175767889 \mathrm{PEL}^{\mathrm{var}} \mathrm{TES}^{\mathrm{var}} \mathrm{SPM}^{\mathrm{var}} \mathrm{SPE}^{\mathrm{ed}}$, excedit $6188^{*} 88^{\text {alt }} \mathrm{AMst}^{\mathrm{var}} \mathrm{PEL}{ }^{\mathrm{var}}$, cadit $\mathrm{AMst}^{\mathrm{AD}}$, cadet $\mathrm{SPM}^{\mathrm{var}}$, decidit $\mathrm{SPE}^{\mathrm{var}}$; + uel cadit 77

siue ] abs. TES SPM SPE; + autem AMst ${ }^{\mathrm{ed}}$, + ergo AMst ${ }^{\mathrm{AD}}$ prophetiae 515458617688 ] abs. TES SPM SPE euacuabuntur ] euacuntur $54^{*}$, abolebuntur $757689 \mathrm{AMst}^{\mathrm{AD}}$, abolebuntur uel euacuabuntur 77, uacuabuntur PEL ${ }^{\text {var }}$, abs. TES SPM SPE siue linguae cessabunt 51545876 ] abs. TES SPM SPE 
siue scientia $545^{8}$ ] siue scientiae $6177 \mathrm{AMst}^{\mathrm{var}} \mathrm{PEL}^{\mathrm{A}}$, abs. TES SPM SPE destruetur $\mathrm{AMst}^{\mathrm{AD}}$ ] destruatur 58 , destruentur $6177 \mathrm{PEL}^{\text {var }}$, euacabitur $\mathrm{AMst}^{\mathrm{ed}}$, euacuabantur AMstrar, abs. TES SPM SPE

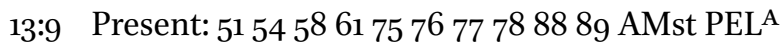
ex parte enim cognoscimus ] scimus $757689 \mathrm{AMst}^{\mathrm{var}} \mathrm{PEL}^{\mathrm{var}}$, scimus uel cognoscimus 77 et ex parte prophetamus $61 A M s t^{e d}$ ] prophetemur 75 , prophetabimus AMstrar

13:10 Present: 51545861757677788889 AMst PEL $^{\mathrm{A}}$

cum autem uenerit ] cum uenerit autem $\mathrm{AMst}^{\mathrm{AD}}$ quod perfectum est ] perfectum 77 euacuabitur $\left(54^{*}\right)$ AMst $^{\text {var }}$ ] destruetur $757^{*}$, destruentur $7^{\mathrm{C}} 89 \mathrm{AMst}^{\mathrm{AD}}$, destruentur uel euacuabuntur 77, euacuabuntur AMst ${ }^{\text {ed }}$, euacabuntur PEL var(ed)

quod ex parte est AMstrar ] quae ex parte sunt $757689 \mathrm{AMst}^{\text {ed }}$, ea quae ex parte 77 , illa quae ex parte sunt $\mathrm{AMst}^{\mathrm{AD}}$, ea quae ex parte sunt $\mathrm{PEL}^{\text {var(ed) }}$

13:11 Present: 51545861757677788889 AMst PEL ${ }^{\mathrm{A}}$

cum essem paruulus 54 ] cum eram paruulus 757689 , cum eram uel essem paruulus 77

loquebar ut paruulus ] ut paruulus loquebar $6175767789 \mathrm{AMst}^{\mathrm{var}} \mathrm{PEL}^{\mathrm{var}(\mathrm{ed})}$, quasi paruulus loquebar AMst ${ }^{\text {ed }}$

sapiebam ut paruulus ] ut paruulus sapiebam $6175767789 \mathrm{PEL}^{\text {var(ed) }}$, quasi paruulus sapiebam AMsted, sicut paruulus sapiebam $\mathrm{AMst}^{\mathrm{AD}}$

cogitabam ut paruulus ] ut paruulus cogitabam 6175767789 PEL ${ }^{\text {var(ed), }}$ quasi paruulus cogitabam $\mathrm{AMst}^{\mathrm{ed}}$, sicut paruulus cogitabam $\mathrm{AMst}^{\mathrm{AD}}$, om. PEL ${ }^{\mathrm{A}}$

quando ] quando autem 5154587888 , cum $617576 \mathrm{AMst}^{\text {ed }} \mathrm{PEL}^{\text {var(ed) }}$, cum autem 7789 , at ubi $\mathrm{AMst}^{\mathrm{R}}$

\section{factus sum uir}

euacuaui quae erant paruuli 51545888 ] quae paruuli erant disposui 61 $\mathrm{PEL}^{\text {var(ed) }}$, quae paruuli erant deposui $75767789 \mathrm{AMst}^{\mathrm{D}}$, ea quae paruuli erant destruxi $\mathrm{AMst}^{\mathrm{ed}}$, qui et paruuli erant destruxi $\mathrm{AMst}^{\mathrm{A} *}$, ea quae paruuli erant deposui $\mathrm{AMst}^{\mathrm{AC}}$, quae paruuli erant euacuaui $\mathrm{PEL}^{\mathrm{var}}$

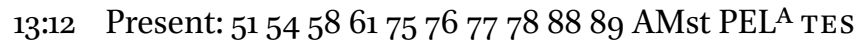

uidemus ] uidimus PEL ${ }^{\text {var }}$ TES $^{\text {var }}$ + enim AMst $^{\mathrm{AD}}$

nunc ] nunc uel modo 77, om. TES ${ }^{\text {var }}$ 
per speculum ] in speculum TES ${ }^{\text {var }}$; et 61 PEL $^{\text {var }}$ TES ${ }^{\text {var }}$

in enigmate 61757789 ] per enigmate TES ${ }^{\mathrm{var}}$

tunc ] nunc uideamus TES ${ }^{\text {var }}$

autem $\mathrm{AMst}^{\mathrm{AD}}$ ] uero $\mathrm{AMst} \mathrm{ed}^{\mathrm{ed}}$ om. PEL var

facie ad faciem 88 TE $^{\text {var }}$ ] faciem ad faciem 757677 TE $^{\text {ed }}$

nunc $\mathrm{AMst}^{\mathrm{AD}}$ ] modo AMst ${ }^{\mathrm{ed}}$; + autem 89 TES $^{\text {var }}$

cognosco ex parte $\mathrm{AMst}^{\mathrm{AD}} \mathrm{TES}^{\mathrm{var}}$ ] scio ex parte $75767789 \mathrm{AMst}^{\mathrm{ed}} \mathrm{TES}^{\text {ed }}$

tunc autem $\mathrm{AMst}^{\mathrm{AD}}$ ] tunc $61 \mathrm{PEL}^{\mathrm{var}}$, tunc uero AMsted, et nunc TES ${ }^{\mathrm{var}}$; + ex

parte 77

cognoscam ] cognoscar TES ${ }^{\text {var }}$

sicut TES $S^{\text {var }}$ ] sicuti TES ${ }^{\text {ed }}$

et TES ${ }^{\text {var }}$ ] om. 6175 AMst $^{\text {A }}$ PEL $^{\text {var }}$ TES $^{\text {ed }}$; + ego 757689 TES $^{\text {ed }}$, + ego super 77 cognitus sum 75

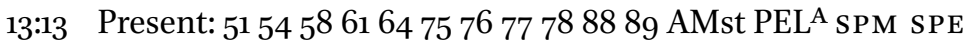

nunc autem manet $\mathrm{SPM}^{\mathrm{var}}$ ] nunc autem manent $51545888 \mathrm{AMst}^{\mathrm{AD}} \mathrm{SPM}^{\mathrm{var}}$ $\mathrm{SPE}^{\mathrm{var}}$, manet 61 PEL ${ }^{\mathrm{var}}$, manet autem $75767789 \mathrm{AMst}^{\mathrm{ed}}$, manent autem nunc AMst ${ }^{\mathrm{R}}$, manent autem AMst ${ }^{\text {var }}$ SPM $^{\text {ed }}$

fides spes caritas $\left.\langle 64\rangle \mathrm{AMst}^{\mathrm{var}} \mathrm{SPM}^{\mathrm{var}}\right]$ spes fides caritas $61 \mathrm{AMst}^{\text {ed }} \mathrm{PEL}^{\mathrm{var}}$ $\mathrm{SPM}^{\mathrm{ed}} \mathrm{SPE}^{\mathrm{var}}$

tria haec 5154586176 ] tria autem haec $\mathrm{SPM}^{\mathrm{var}}$

maior autem ] maior $\mathrm{AMst}^{\mathrm{AD}} \mathrm{SPM}^{\text {var }}$

his est ] horum est $5889 \mathrm{AMst}^{\mathrm{ed}} \mathrm{SPM}^{\mathrm{ed}}$, horu[m ... 64, horum $7576 \mathrm{sPM}^{\text {var, }}$, his uel horum 77, eorum est AMst ${ }^{\text {var }}$, est horum $\mathrm{AMst}^{\mathrm{AD}}$, his PEL var caritas ] lac. 64

14:1 Present: $51545^{8} 61647576777888$ 89 AMst PEL ${ }^{A}$ SPM SPE sectamini caritatem $\langle 64\rangle]$ sectamini autem caritatem $S P E^{\text {var }}$ aemulamini spiritalia 5154586188 ] aemulamini autem spiritalia $\langle 64\rangle 7576$ 777889 , abs. SPM SPE

magis autem ] abs. SPM SPE

ut prophetetis $A M s t^{e d}$ ] ut probetis $54^{*}$, ut prophetizetis 58 , ut prophetis 61 $78^{*}$ AMst $^{\text {var }}, \mathrm{u}[\ldots] 64$, abs. SPM SPE

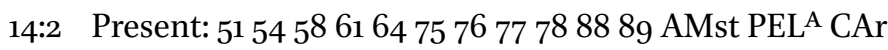

qui enim ] quia enim $\mathrm{AMst}^{\mathrm{var}}$, nam qui $\mathrm{AMst}^{\mathrm{R}}$

loquitur lingua $\left.\mathrm{AMst}^{\mathrm{var}}\right]$ loquitur linguis $77^{\mathrm{C}} \mathrm{AMst}^{\mathrm{ed}}$

non hominibus loquitur sed deo ] [...] loqu[i] tur sed deo 64

nemo enim $\langle 64\rangle]$ abs. CAr

audit ] audẹt $54^{*}$, audịt $54^{\mathrm{C}}$, lac. 64 , abs. CAr 
spiritu ] spiritus 515475767789 AMst $^{\mathrm{A}}$ PEL var, lac. 64, abs. CAr autem loquitur $\langle 64\rangle]$ autem loqui $\mathrm{PEL}^{\mathrm{var}}$, abs. CAr mysteria $545^{8} 6188^{*}$ ] mysterium $\mathrm{AMst}^{\mathrm{AD}}$, abs. CAr

14:3 Present: 5154586164757677788889 AMst PEL $^{\mathrm{A}}$

nam qui ] qu[...] 64, si enim qui 77 , qui autem $\mathrm{AMst}^{\mathrm{AD}}$, qui enim $\mathrm{AMst}^{\mathrm{ed}}$ prophetat hominibus loquitur $67\langle 64\rangle]+\operatorname{ad} 515458617888^{\mathrm{C}} \mathrm{AMst}^{\mathrm{ed}} \mathrm{PEL}^{\mathrm{var}}$ aedificationem $51545^{8}\langle 64\rangle 7_{688^{c}} \mathrm{PEL}^{\mathrm{var}}$ ] aedificatione $\mathrm{PEL}^{\mathrm{var}}$

et exhortationem 5458617888 ] uel consolationem $77^{\mathrm{C}}$, et exhortatione $\mathrm{PEL}^{\mathrm{var}}$; + uel consolationem $77^{*}$

et consolationes PEL ${ }^{\text {var }}$ ] et consolationem 5154586175767889 AMst PEL $^{\mathrm{A}}$, et cons [8-9] 64, et exhortationem 77 , et consolatione PEL var

14:4 Present: $5^{1} 545^{8} 6164757^{6} 777_{8} 8488$ 89 AMst PEL ${ }^{\mathrm{A} \dagger}$

qui ] fratres qui 84, om. PEL ${ }^{\text {var }}$ + enim 54

loquitur lingua ] loquitur linguis 84

semet ipsum AMst ${ }^{\text {var }}$ ] se ipsum 586475767789 AMst $^{\text {ed }}$

aedificat 515458767788 ] lac. 64 , abs. 84

qui autem $\left.\mathrm{AMst}^{\mathrm{AD}}(61)\right]$ qui $54^{*}$, qui uero $77 \mathrm{AMst}^{\mathrm{ed}}$, quiș autem $78^{*}$, abs. 84 prophetat 61 ] abs. 84

ecclesiam $6178(88)$ ] ecclesiam dei 515877 , eccles[...] 64, abs. 84

aedificat 5154587688 ] lac. 64 , abs. 84

14:5 Present: 5154586164757677788889 AMst PEL ${ }^{\mathrm{A}}$

uolo autem ] uolo enim 7576 , uolo 89

omnes uos loqui linguis $\left.\mathrm{AMst}{ }^{\mathrm{AD}} \mathrm{PEL}^{\mathrm{var}}\right]$ uos loqui linguis ${ }_{51} \mathrm{PEL}^{\mathrm{A}}$, uos linguis loqui omnes 61 PEL var, uos omnes lingu[is loqui] 64, uos omnes loqui linguis 8889 AMst $^{\text {ed }} \mathrm{PEL}^{\text {var }}$

magis autem $\langle 64\rangle$

prophetare $6 \mathrm{r}$ ] ut prophetetis $647^{6} 77^{8} 9^{\mathrm{C}} \mathrm{AMst}$, ut prophetis $7589^{*}$

nam maior est ] maior est enim $61757689 \mathrm{AMst}^{\text {ed }} \mathrm{PEL}^{\mathrm{var}}$, lac. 64, maior enim est 77 , maior est $\mathrm{AMst}^{\mathrm{D} *}$

qui prophetat quam qui $6 \mathrm{r}$ ] qui prophetat quam $\mathrm{AMst}^{\mathrm{var}}$

loquitur linguis ] lin[...] 64, loquitur lingua $77^{*} 89 \mathrm{AMsted}$, lingua loquitur $\mathrm{AMst}^{\mathrm{var}}$, linguis loquitur PEL var

nisi si ] nisi $5^{1} 545^{8} 617888 \mathrm{AMst}^{\text {ed }} \mathrm{PEL}$, lac. 64, nisi qui 77 , nisi aliquis $\mathrm{AMst}^{\mathrm{AD}}$

$\dagger{ }_{1}$ Cor. 14:4. The second half of this verse is absent from the Pseudo-Jerome tradition of PEL. 
forte ut ] forte $5154^{\mathrm{C}} 7888^{*}$, lac. 64 , om. 75767789 AMst, ut forte PEL var interpretetur 7589 ] lac. 64, interpretatur PEL var, om. PEL var ut ecclesia aedificationem 54586176 ] ut aedificationem 51, lac. 64, om. AMst ${ }^{\mathrm{var}}$

accipiat ] lac. 64, habeat accipiat 77, om. AMstvar

14:6 Present: 51545861757677788889 AMst PEL ${ }^{\mathrm{A}}$

nunc autem AMst ${ }^{\mathrm{var}}$ ] nunc AMst ${ }^{\text {ed }}$, nunc uero AMstrar

fratres si uenero ad uos linguis loquens 54

quid uobis ] quid 61 PEL var

prodero ] prode ero 7589 , proderit 76 PEL var

nisi si PEL ${ }^{\text {var }}$ ] nisi $5^{1} 5^{8} 617576778889$ AMst $^{\mathrm{ADR}} \mathrm{PEL}^{\mathrm{A}}$, si non $\mathrm{AMst}^{\mathrm{ed}}$ uobis loquar $\mathrm{AMst}^{\mathrm{AD}}$ ] loquar uobis $\mathrm{AMst}^{\mathrm{ed}}$, loquor uobis $\mathrm{AMst}^{\mathrm{var}}$ aut ] om. $61757689 \mathrm{AMst}^{\mathrm{AD}} \mathrm{PEL}^{\text {var }}$

in reuelatione 58 ] in reuelationem $617576 \mathrm{PEL}^{\mathrm{var}}$, ad apertionem 89 aut ] ut 75 , aut et PEL var; + in 5154587778 AMst PEL var

scientia $5888 \mathrm{AMst}^{\mathrm{ADR}}$ ] scientiam 89, notitia AMsted aut ] + in $5^{8} 77$ AMst PEL var

prophetia 5861 ] prophetiam 89 aut in doctrina $\left.\mathrm{PEL}^{\mathrm{var}}\right]$ aut doctrina $5^{1} 6188^{\mathrm{C}} \mathrm{PEL}^{\mathrm{A}}$, aut doctrinam 89

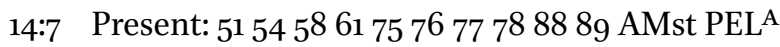

tamen quae 51545861767788 ] tamen qui $75 \mathrm{AMst}^{\mathrm{A}}$, tamen qua 89 sine anima sunt $\left.\left(\mathrm{PEL}^{\mathrm{A} *}\right)^{\mathrm{C}}\right]$ sine anima 77 , animam non habent $\mathrm{AMst}^{\mathrm{AD} *}$ uocem dantia ] uocem dant 75 , uocem dantia sunt $\mathrm{AMst}^{\mathrm{AD}}{ }^{\mathrm{A}}$ siue tibia siue cithara 51588889 nisi $\mathrm{AMst}^{\mathrm{var}}$ ] si $\mathrm{AMst}^{\mathrm{ed}}$, si non $\mathrm{AMst}^{\mathrm{AD}}$ distinctionem (75)

sonituum ] sonitus $5^{1} 5^{8} 6189^{\mathrm{C}} \mathrm{PEL}^{\mathrm{var}(\mathrm{ed})}$, sonitum $54^{*} 88$, soni 7576 , et phthongis 77 , sonuus $89^{*}$, sonus AMst ${ }^{\text {ed }}$, per sonos $\mathrm{AMst}^{\mathrm{AD}}$

dederint $\mathrm{AMst}^{\mathrm{var}}$ ] dent 757689 , dederunt 77 , non dederint AMst ${ }^{\text {ed }}$, non dederunt $\mathrm{AMst}^{\mathrm{R}}$, dederit $\mathrm{PEL} \mathrm{L}^{\text {var }}$

quomodo scietur $\mathrm{AMst} \mathrm{AD}^{\mathrm{AD}}$ ] quomodo cognoscetur $\mathrm{AMst}^{\mathrm{ed}}$ quod ] id quod 54586188 PEL $^{\text {var }}$, aut quod PEL var

canitur ] per tibiam canitur 617576 AMst PEL var, tibicinaturale uel quod per tibiam canitur 77 , per tibia canitur 89, tibia canitur PEL ${ }^{\text {var(ed) }}$

aut quod ] uel quod $7576 \mathrm{AMst}^{\mathrm{AD}}$

citharizatur 51546189 ] + uel citharizatum 77 


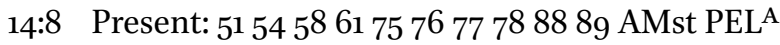

etenim si ] nam si $757_{6} \mathrm{AMst}^{\mathrm{AD}}$, etenim uel nam si 77 , nam et si 89 , et si enim si AMstrar

\section{incertam uocem}

det tuba 5478 ] de tuba $5^{1}\left(88^{*}\right)$, om. 75 , tuba dederit 7689 AMst ${ }^{\text {ed }}$, sapix uel tuba dederit 77 , dederit tuba AMstrar, tuba det $78^{{ }^{\mathrm{gl}}} \mathrm{PEL}{ }^{\mathrm{var}}$

quis ] quomodo quis $61 \mathrm{PEL}{ }^{\text {var }}$, om. $75 ;+[\ldots] 88^{*}$

parabit se ] parauit se 5489 , praeparauit se $6 r^{*}$, praeparabit se $6 r^{c} \mathrm{PEL}^{\mathrm{var}}$, om. 75 , se praeparabit AMst ${ }^{\text {ed }}$, se parabit AMstvar

ad bellum] om. 75, ad proelium AMsted, bellum AMst ${ }^{\text {var }}$

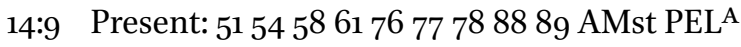

ita et uos ] ita et nos PEL var

per linguam nisi manifestum sermonem ] quomodo scietur id quod dicitur

nisi 58 , per linguam nisi significantem sermonem 7689 AMst, per linguam nisi significantem uel significatum sermonem 77

dederitis ] + uos 58

quomodo scietur ] om. 58 , quomodo intellegatur $\mathrm{AMst} \mathrm{AD}^{\mathrm{AD}}$

id quod dicitur 54 ] per linguam manifestum sermonem 58 , quod dicitur 76 $89 \mathrm{PEL}^{\text {var, }}$, locutio uel quod dicitur 77 , quid loquimini AMst ${ }^{\text {ed }}$, quod loquamini $\mathrm{AMst}^{\mathrm{var}}$, quid dicitur $\mathrm{AMst} \mathrm{AD}^{\mathrm{AD}}$

eritis enim in

aera loquentes ] aere loquentes $8888^{\text {alt } \dagger} \mathrm{AMst}^{\mathrm{var}}$

14:10 Present: 515458617677788889 AMst PEL ${ }^{A}$

tam multa $\mathrm{AMst}^{\mathrm{R}}$ ] tanta $61 \mathrm{PEL}^{\text {var(ed) }}$, tantum 76, om. $7789 \mathrm{AMst}^{\mathrm{var}}$, multa AMsted

ut puta ] ut pote $77 ;+$ quanta $\mathrm{AMst}^{\mathrm{AD}}$

genera linguarum sunt 78 ] linguarum genera sunt $61 \mathrm{PEL}^{\mathrm{var}}$, genera sunt 76 , genera uocum id est linguarum sunt 77 , genera uocum sunt $78^{\mathrm{gl}}$, genera sunt linguarum PEL var

in mundo ] in hoc mundo 5154586176788889 AMst PEL ${ }^{\text {var }}$

et nihil 5154 ] + eorum 6177 PEL $^{\text {var }}$

sine uoce est ] sine uoce 76 AMst PEL var

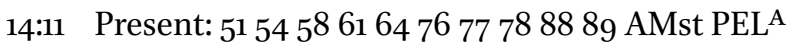

si ergo ] lac. 64, igitur si $89 \mathrm{AMst}^{\mathrm{AD}}$

$\dagger{ }_{1}$ Cor. 14:9. The text of the alternative reading in VL 88 is not clear: it may just be ere. 
nesciero ] lac. 64, ignorem $76 \mathrm{AMst}^{\mathrm{AD}}$

uirtutem uocis ero ] lac. 64

ei cui loquor ] cui loquor 51, lac. 64, loquenti 77, ei qui loquitur 89

barbarus ] lac. 64

et qui $\mathrm{AMst} \mathrm{AD}^{\mathrm{AD}}$ ] et is qui $6177 \mathrm{AMst}^{\mathrm{ed}} \mathrm{PEL}^{\mathrm{var}}$, [e]t ille qui 64, et his qui 89

loquitur mihi $5^{1}$ ] loquitur uel loquens mihi 77 , loqui loquitur mihi 88 , mecum loquitur PEL var

barbarus $\langle 64\rangle$

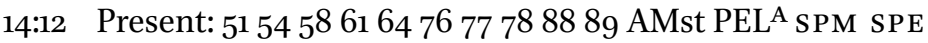
sic ] lac. 64, ita $76 \mathrm{AMst}^{\mathrm{AD}}$, sic uel ita 77 , abs. SPM SPE

et uos quoniam ] lac. 64, et uos quia AMst ${ }^{\mathrm{var}}$, om. $\mathrm{SPM}^{\mathrm{var}}$, $\langle a b s$.$\rangle quoniam$ SPE

aemulatores estis 5154586176777888$]$ [aem] ulatores estote 64 , om. SPM ${ }^{\text {var }}$ spirituum ] spiritalium 61 $\langle 64\rangle \mathrm{PEL}^{\text {var(ed) }} \mathrm{SPM}^{\mathrm{ed}}$, spiritum 76 , spiritus $\mathrm{AMst}^{\mathrm{AD}}$, om. SPM $^{\mathrm{var}}$; + uel spiritalium 77

ad ] om. $54 \mathrm{AMst}^{\mathrm{AD}} \mathrm{sPM}^{\mathrm{var}}$, lac. 64

aedificationem ecclesiae $51545861\langle 64\rangle 76(78) 88] \mathrm{om}$. $\mathrm{SPM}^{\mathrm{var}}$

quaerite ut $5154\langle 58\rangle 61\langle 64\rangle 7678] \mathrm{om}$. $\mathrm{SPM}^{\mathrm{var}}$

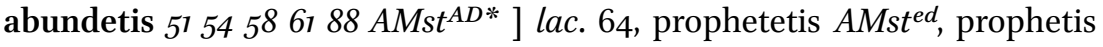
$\mathrm{AMst}^{\mathrm{var}}$, om. $\mathrm{SPM}^{\mathrm{var}}$

14:13 Present: $5^{1} 545^{8} 616476777888$ 89 AMst PEL ${ }^{A}$ CAr

et ideo ] propterea 64, om. $76 \mathrm{AMst}^{\text {var }}$, propter quod 7789 , ideo AMst ${ }^{\text {ed }} \mathrm{CAr}$ qui loquitur ] om. AMst ${ }^{\text {var; }}+$ uel loquens 77

lingua oret ut interpretetur $\langle 64\rangle 77\left(78^{*}\right) 8889$

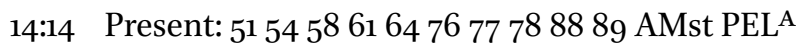

nam si $\mathrm{AMst}^{\mathrm{R}}$ ] si enim 6476 , si $7789 \mathrm{AMst}^{\text {ed }} \mathrm{PEL}^{\text {var }}$

orem ] orauero 61647677 AMst PEL ${ }^{\text {var }}$, oret 89

lingua ] lac. $64 ;+$ mea 89

spiritus meus orat $\langle 64\rangle$

mens autem $\langle 64\rangle]$ sed mens $76 \mathrm{AMst}^{\mathrm{AD}}$, manus autem $\mathrm{AMst}^{\text {tar }}$

mea sine fructu est $\left(54^{*}\right)\langle 64\rangle$

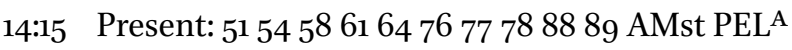

quid ergo est $\mathrm{AMst} \mathrm{AD}^{\mathrm{AD}}$ ] quid est ergo $89^{*} \mathrm{AMst}^{\mathrm{ed}}$, quid est PEL ${ }^{\text {var }}$

orabo $\left.\left(5^{8}\right)\right]+$ et $88^{*}$

spiritu ] + id est uoce 77

orabo et $\langle 64\rangle]$ orabo PELvar 
mente ] mentem 89

psallam spiritu ] om. $54^{*}$, psalmum dicam spiritu 89 AMst; + id est uoce 77 psallam $\langle 64\rangle$ ] om. $54^{*}$, psalmum dicam 89 AMst

et mente $\langle 64\rangle$ ] om. $54^{*}$, et mentem 89

14:16 Present: $51545^{861647677788889 \text { AMst PEL }}{ }^{\mathrm{A}}$

ceterum si 61 ] alioquin si 64

benedixeris spiritu $\langle 76\rangle]$ benedixeritis spiritu $5^{1} 61$ PEL ${ }^{\text {var }}$, bene[...] 64

qui AMst ${ }^{\text {var }}$ ] quis $51545^{8} 7788^{\mathrm{C}} 89 \mathrm{AMst}^{\mathrm{ed}} \mathrm{PEL}^{\text {var(ed) }}$, quid 61 PEL ${ }^{\mathrm{var}}$, lac. 64 supplet locum idiotae 5458647788 ] supplebit locum idiotae 51

quomodo $\langle 64\rangle(88)$ ] aut quomodo 54

dicet $\mathrm{AMst}^{\mathrm{R}}$ ] dicit $5488 \mathrm{AMst}^{\mathrm{ed}} \mathrm{PEL}$, lac. 64, dicent 89

amen super ] [a]men in 64 , amen supra 89

tuam benedictionem ] tua benedictione 54 , tuam gratiam uel benedictionem 77 , tuam eucharistiam AMst ${ }^{\mathrm{AD}}$

quoniam ] quam 51, quoniam quidem $617789 \mathrm{AMst}^{\mathrm{AD}} \mathrm{PEL}^{\mathrm{var}(\mathrm{ed})},[\ldots] \mathrm{em} 64$, quia AMst $\mathrm{ed}^{\mathrm{ed}}$

quid dicas nescit $54^{*}$ ] nescit quid dicas $6176 \mathrm{AMst}^{\text {ed }} \mathrm{PEL}^{\text {var(ed) }}$, nescit quid dicis 64 77, quid dicis nescit 88, nescit quid dices 89, nescit quid dicet AMst var

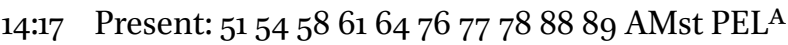

nam tu quidem ] tu quidem $\langle 64\rangle 76 \mathrm{PEL}^{\mathrm{var}}$

bene gratias agis $\left.58\langle 64\rangle(77)\left(88^{*}\right)\right]$ bene gratias ages PEL var

sed alter ] sed alius 647789 AMst

non aedificatur $515458\langle 64\rangle 7688$ ] qui aedificatur AMst ${ }^{\text {var }}$

14:18 Present: $5^{1} 545^{8} 6164757677788889$ AMst PELA

gratias ago $][\ldots]$ ago 64 , lac. 75 , gratias autem PEL var

deo ] lac. 75 , om. 78 ; + meo 58 AMst $^{\text {ed }}$

quod ] om. 64, quia $\mathrm{AMst}^{\mathrm{AD}}$

omnium uestrum $\langle 64\rangle$

lingua ] magis lingua $61 \mathrm{AMst}^{\mathrm{AD}} \mathrm{PEL}^{\text {var(ed) }}$, lac. 64, linguam aliis 7576 , lingua magis 77 , magis linguam $P E L^{\text {var }}$

loquor ] lac. 64, loquar $757^{\mathrm{C}} \mathrm{PEL}$ var

14:19 Present: $5^{1} 545^{8} 61757677788889$ AMst PEL $^{\mathrm{A}}$

sed ] om. AMst $\mathrm{D}^{\mathrm{D}}$

in ecclesia 6178 ] in ecclesiam PEL var

uolo ] magis uolo $54^{\mathrm{C}} 5^{8}$ 
quinque uerba ] quinque sermones uel uerba 77

sensu meo loqui $\mathrm{AMst}{ }^{\mathrm{AD}}$ ] loqui per sensum meum $7576 \mathrm{AMst}^{\mathrm{R}}$, loqui qui-

dem mente mea 77 , loqui sensu meo 78 , loqui quidem sensu meo $7^{\mathrm{gl}}$, loqui $89 \mathrm{AMst}^{\text {ed }}$, cum sensu meo loqui PEL ${ }^{\text {var }}$, loqui meo sensu PEL var

\pm per legem $6189 \mathrm{AMst}^{\text {ed }} \mathrm{PEL}^{\text {var(ed) }}$

ut et ] ut $54^{*} 61$, om. PEL var

alios instruam ] alios aedificem AMst, om. $\mathrm{PEL}^{\mathrm{var}}$

quam decem milia ] quam multa milia $757689 \mathrm{AMst}^{\mathrm{AD}}$

uerborum ] sermonum uel sermones 77 , sermonum $\mathrm{AMst}^{\mathrm{AD}}$, om. $\mathrm{PEL}^{\text {var }}$ in lingua ] om. $51 ;+$ obscura $58,+$ uerborum PEL var

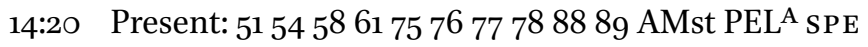

fratres ] + mei 5478

nolite pueri effici sensibus sed malitia paruuli ${ }_{57}(54) 58758889$

estote ] sitis 58 , om. PEL var

sensibus autem perfecti estote (88*) ] ut sensibus perfecti sitis 61757677 7889 AMst, ut sensibus perfecti PEL ${ }^{\text {var(ed) }}$

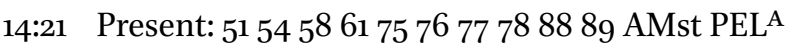

in lege ] + enim 58618889 AMst $^{\text {ed }}$ PEL $^{\mathrm{A}}$

scriptum est ] scriptura est $\mathrm{AMst}^{\mathrm{R}}$

quoniam in aliis linguis $\mathrm{AMst}^{\mathrm{AD}}$ ] quia in aliis linguis $\mathrm{AMst}^{\mathrm{ed}}$

et ] om. $\mathrm{AMst}^{\mathrm{AD*}}$; + in $757677 \mathrm{AMst}^{\mathrm{ed}}$

labiis aliis ] labiis $89 \mathrm{PEL}^{\mathrm{var}}$, aliis labiis $\mathrm{AMst}^{\mathrm{var}}$, om. $\mathrm{AMst}^{\mathrm{AD} *}$

loquar ] loquor PEL var

populo huic ] populo 75, plebi huic $\mathrm{AMst}^{\mathrm{AD}}$

et nec sic $\mathrm{AMst}^{\mathrm{ADR}}$ ] et ut sic 58 , et nondum $75767789 \mathrm{AMst}^{\text {ed }} \mathrm{PEL}^{\mathrm{var}}$

exaudient me ] audient me 58 , exaud[ient me $] 75$, exaudiet me $7788^{*} 89$, me

exaudiet $\mathrm{AMst}^{\mathrm{ed}}$, me audient $\mathrm{AMst}^{\mathrm{R}}$, me exaudient $\mathrm{AMst}^{\mathrm{var}}$

dicit dominus

14:22 Present: 51545861757677788889 AMst PEL $^{\mathrm{A}}$

itaque linguae in 51545877

signum sunt $(75)]$ signo sunt $88 \mathrm{AMst}^{\mathrm{var}}$, signos $\mathrm{AMst}^{\mathrm{var}}$

non $\left.\mathrm{AMst}^{\mathrm{AD}}\right]+$ utique $\mathrm{AMst}^{\mathrm{ed}}$

fidelibus ] his qui credunt 757689 AMst $^{\text {ed }}$, iis qui credunt $\mathrm{AMst}^{\mathrm{R}}$; + uel credentibus 77

sed infidelibus ] sed non credentibus 75767789 AMst; + uel infidelibus 77 prophetia $A M s t^{e d} \mathrm{PEL}^{\mathrm{var}}$ ] prophetiae $5158617888 \mathrm{AMst}^{\mathrm{var}} \mathrm{PEL}^{\mathrm{A}}$

autem non infidelibus $\mathrm{AMst}^{\mathrm{AD}}$ ] autem non incredulis $\mathrm{AMst}^{\mathrm{ed}}$ 
sed fidelibus $\mathrm{AMst} \mathrm{tD}^{\mathrm{AD}}$ ] sed infidelibus $54^{*}$, sed his qui credunt AMst ${ }^{\mathrm{ed}}$, sed iis qui credunt $\mathrm{AMst}^{\mathrm{R}}$, om. PEL var

14:23 Present: 515458617576777888 89 AMst PEL A $^{\mathrm{A}}$

si ergo $\mathrm{AMst}^{\mathrm{AD}}$ ] si $75767789 \mathrm{AMst}^{\mathrm{ed}}$

conueniat uniuersa ] conuenerit uniuersa 75 (76) $7789 \mathrm{AMst}^{\text {ed }}$, uniuersa conuenerit $\mathrm{AMst}^{\mathrm{AD}}$, conueniant uniuersa PEL var

ecclesia in unum $5^{1} 6188$ ] + uel in id ipsum 77

et omnes linguis loquantur ] et loquantur linguis omnes 7576 , et omnes loquantur linguis 77 , et linguis loquantur omnes $89 \mathrm{AMst}^{\mathrm{AD}}$, et loquantur omnes linguis AMst ${ }^{\text {ed }}$, et omnes linguas loquantur PEL var

intrent $\mathrm{AMst}^{\mathrm{AD}}$ ] introeant $\mathrm{AMst}^{\mathrm{ed}}$, intret $\mathrm{PEL} \mathrm{L}^{\text {var }}$

autem $\mathrm{AMst}^{\mathrm{AD}}$ ] autem et $54 \mathrm{AMst}^{\mathrm{ed}} \mathrm{PEL}{ }^{\mathrm{var}}$, et $\mathrm{AMst}^{\mathrm{var}}$, om. AMst $^{\mathrm{R}}$

idiotae aut infideles $51545^{8}\left(61^{*}\right) 767888$ ] idiotae aut infidelis 75 PEL $^{\text {var, }}$, idiotae $89 \mathrm{AMst}^{\mathrm{ed}}$, imperiti aut infideles $\mathrm{AMst}^{\mathrm{AD}}$, infideles et idiotae $\mathrm{AMst}^{\mathrm{R}}$, idiotae uel infideles PEL ${ }^{\mathrm{A}}$

nonne ] non AMst ${ }^{\text {var }}$ PEL ${ }^{\text {var }}$, om. PEL var

dicent ] dicit 89, om. PEL var

quod insanitis ] quid insanitis 51 , quia insanitis 75767789 AMst, numquid insanitis PEL var

14:24 Present: 51545861757677788889 AMst PEL ${ }^{A}$

si autem omnes prophetent 61 ] quod si omnes prophetent AMst ${ }^{\text {var }}$

intret autem ] introierit autem AMst ${ }^{\text {var }}$

quis ] aliquis 61 AMst PEL var

infidelis uel idiota $51545^{8} 88$ ] infidelis aut idiota $617576777889^{\mathrm{C}} \mathrm{PEL}^{\mathrm{var}}$, infidelis aut idiotae 89 , infidelis aut imperitus $\mathrm{AMst}^{\mathrm{AD}}$, idiota uel infidelis PEL var(ed)

conuincitur ] conuincetur 54, increpatur AMst ${ }^{\text {ed }}$, arguitur AMst ${ }^{\mathrm{AD}}$

ab omnibus ] ab hominibus PEL var

diiudicatur PELvar(ed) ] deiudicatur 61 PEL ${ }^{\mathrm{A}}$, interrogatur 757677 89, redarguitur AMst ${ }^{\text {ed }}$, iudicatur AMst ${ }^{\mathrm{AD}}$

ab omnibus

14:25 Present: $51545^{8} 61647576777888$ 89 AMst PEL A $^{\mathrm{A}}$

occulta 58 ] lac. 64

\pm etiam 54757689 , quoque $\mathrm{AMst}{ }^{\mathrm{AD}}$, autem PEL ${ }^{\text {var }}$

cordis eius $\left(78^{*}\right)$ ] lac. 64 , cordi eius $88 ;+$ ei $88^{\mathrm{C}}$

manifesta fiunt AMst ${ }^{\text {var }}$ ] manifesta sunt 51, manifesta fient $547576 \mathrm{AMst}^{\mathrm{ed}}$, lac. 64, manifesta fiant 89 
et ita ] et tunc 5461757689 AMst PEL ${ }^{\text {var }}$, lac. 64

cadens in faciem ] cadens in terram $61 \mathrm{PEL}^{\mathrm{var}},[\ldots] \mathrm{f}[$ aciem $] 64$

adorabit $88^{*}$ ] lac. 64 , adorauit 7589 , orabit $\mathrm{AMst}^{\mathrm{D} *}$

deum ] d $[\ldots] 64$, dominum PEL var

pronuntians $51545^{8}(61)$ ] lac. 64 , adnuntians $757677^{*} 89$, pernuntians $77^{\mathrm{C}}$

quod ] lac. 64, quia $\mathrm{AMst}^{\mathrm{D}}$

uere ] u[3] 64, uero 89

deus in uobis est ] deus in uobis sit $51545^{8} 6178 \mathrm{PEL}^{\mathrm{A}}, \mathrm{d}[\ldots]$ 64, deus in nobis sit 88 , deus est in uobis 89 PEL $^{\text {var(ed) }}$, in uobis deus sit $\mathrm{AMst}^{\mathrm{R}}$

14:26 Present: $5^{1} 545^{8} 61647576777888$ 89 AMst PEL ${ }^{A}$ CAr SPM SPE

quid ergo est $54 \mathrm{SPM}^{\text {var }}$ ] quid ergo $5^{1} 61 \mathrm{PEL}^{\text {var }}$, lac. 64, quid est ergo AMst ${ }^{\mathrm{ed}}$, quid igitur est $\mathrm{AMst}^{\mathrm{A}}$, quid est igitur $\mathrm{AMst}^{\mathrm{R}}, a b s . \mathrm{SPM}^{\mathrm{ed}}$

fratres $\mathrm{SPM}^{\mathrm{var}}$ ] lac. 64, abs. $\mathrm{SPM}^{\mathrm{ed}}$

$\operatorname{cum}\langle 64\rangle]$ dum $5^{8}$

conuenitis ] lac. 64, uenitis $\mathrm{AMst}^{\mathrm{var}}$, conueneritis $\mathrm{AMst}^{\mathrm{AD}}$; + in unum 61

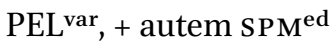

unusquisque uestrum ] lac. 64, unusquisque $\mathrm{AMst}^{\mathrm{AD}} \mathrm{PEL}^{\mathrm{var}}$

psalmum habet $\langle 64\rangle 88$ ] canticum habet $\mathrm{AMst}^{\mathrm{R}}$, om. PEL ${ }^{\mathrm{var}}, a b s$. CAr

doctrinam habet PEL ${ }^{\text {var(ed) }}$ ] lac. 64, om. PEL ${ }^{\mathrm{A}}$, abs. CAr

apocalypsin habet $5^{1} 5458617888$ ] reuelationem habet $\langle 64\rangle 75\langle 76\rangle 77$ $\mathrm{AMst}^{\mathrm{ed}}$, adapertionem habet $\mathrm{AMst}{ }^{\mathrm{AD}}$, om. sPM, abs. CAr

linguam habet $\mathrm{PEL}^{\mathrm{var}}$ ] lac. 64, linguam habent 75, interpretationem habet $89 \mathrm{PEL}^{\text {var(ed) }} \mathrm{SPM}^{\mathrm{var}}$, om. PEL ${ }^{\mathrm{A}} \mathrm{SPM}^{\mathrm{ed}}$, abs. CAr

interpretationem habet $5^{8}\langle 64\rangle 7588 \mathrm{PEL}^{\mathrm{var}}$ ] linguam habet $89 \mathrm{PEL}^{\text {var(ed) }}$ SPM $^{\text {var }}$, om. PEL ${ }^{\mathrm{A}}$, abs. CAr

omnia ad aedificationem fiant $515458\langle 64\rangle 767788$ ] abs. CAr; + uobis s PM ${ }^{\text {ed }}$

14:27 Present: 51545861647576777888 89 AMst PEL $^{\mathrm{A}}$

siue ] lac. 64, si AMst ${ }^{\text {var }}$ + enim AMst ${ }^{\text {ed }}$

lingua quis loquitur ] lingua quis loquatur 54, lac. 64

secundum duos $][\ldots] \mathrm{d}[\ldots]$ 64, per binos $757689 \mathrm{AMst}^{\mathrm{AD}}$, per duos AMst $\mathrm{t}^{\text {ed }}$ aut ut ] aut $5^{1} 54^{*} 5^{8} 6175767888 \mathrm{AMst}^{\text {ed }} \mathrm{PEL}$, lac. 64, siue $\mathrm{AMst}{ }^{\mathrm{AD}}$, ut $\mathrm{AMst}^{\mathrm{var}}$

multum tres ] lac. 64, multum uel plurimum tres 77

et $\langle 64\rangle]$ om. PEL var

per partes $\mathrm{AMst}^{\mathrm{AD}}$ ] lac. 64, particulatim $\mathrm{AMst}^{\mathrm{ed}}$, per particulatim $\mathrm{AMst}^{\mathrm{var}}$ et $\left.\left(75^{*}\right) \mathrm{AMst}^{\mathrm{var}}\right]$ lac. 64 , om. $88^{\mathrm{C}} \mathrm{AMst}^{\mathrm{AD}}$, ut $89 \mathrm{AMst}^{\mathrm{ed}}$

unus interpretetur $75 * 88$ ] lac. 64 


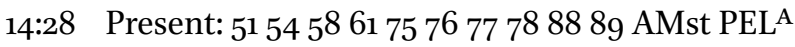
si autem $\mathrm{AMst}^{\mathrm{AD}}$ ] si 89, quod si AMsted non fuerit interpres ] non erit interpres PEL ${ }^{\text {var }}$ taceat in ecclesia 6177 ] taceat in ecclesiam 7576 sibi autem ] si autem $54^{*}$, sibi PEL var loquatur et deo

14:29 Present: 51545861757677788889 AMst PEL ${ }^{\mathrm{A}}$ prophetae 51545861757688 ] + autem $77 \mathrm{AMst}^{\text {ed }} \mathrm{PEL}^{\mathrm{var}}$ duo aut tres ] duo uel tres $6175767789 \mathrm{AMst}^{\mathrm{ADR}} \mathrm{PEL}^{\mathrm{var}}$ dicant ] loquantur 757677 AMst et ceteri $61 \mathrm{AMst}^{\mathrm{AD}}$ ] et alii $\mathrm{AMst}{ }^{\mathrm{ed}}$; + uel alii 77 diiudicent ] examinant 61, exaniment 75 , examinent 7689 PEL ${ }^{\text {var }}$, deiudicent uel examinent 77, examinent uel interrogent AMst, interrogent PEL var(ed)

14:30 Present: 51545861757677788889 AMst PEL ${ }^{\mathrm{A}}$ quod si ] quod si $54^{\mathrm{C}}$, quod alii 58 , si alii 77 reuelatum fuerit sedenti prior taceat

14:31 Present: 51545861757677788889 AMst PEL ${ }^{A}$ potestis enim omnes per singulos prophetare PEL ${ }^{\text {var }}$ ] per singulos omnes prophetare $6 r$ $89 \mathrm{AMst}^{\text {ed }} \mathrm{PEL}^{\text {var(ed) }}$, per singulos prophetare $\mathrm{AMst}^{\mathrm{var}} \mathrm{PEL}^{\mathrm{A}}$ ut omnes discant ] ut omnes dicant $54^{*}$, et omnes discant 89 et omnes exhortentur 546177788889 ] exhortent AMst ${ }^{\mathrm{var}}$, consolentur AMst ${ }^{\mathrm{AD}}$

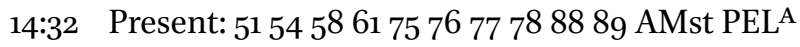
et spiritus prophetarum prophetis $\left\langle 5^{8}\right\rangle{ }^{6} \mathrm{r} A M s t$ subiecti sunt ] subiectus est $5^{1}\left\langle 5^{8}\right\rangle 6175767789$ AMst PEL ${ }^{\text {var(ed) }}$, subiecti $[3-4] 54^{*}$

14:33 Present: $51545^{8} 61757677788889$ AMst $^{\dagger}$ PEL $^{\mathrm{A}}$

non enim est ] non enim $5189 \mathrm{AMst}^{\mathrm{A}} \mathrm{PEL}^{\mathrm{var}}$, non est enim AMsted $\mathrm{PEL}^{\mathrm{var}}$, non est PEL var dissensionis 5861

$\dagger{ }_{1}$ Cor. 14:33. The first half of this verse is missing from five manuscripts of AMst. 
deus ] deus est $89 \mathrm{PEL}^{\text {var }}$, deus res $\mathrm{AMst} \mathrm{AD}^{\mathrm{AD}}$, auctor $\mathrm{AMst} \mathrm{t}^{\mathrm{R}}$, res $\mathrm{AMs} \mathrm{t}^{\mathrm{ed}}$ sed pacis sicut in omnibus ecclesiis ${ }_{51} 61$ ] ecclesiis PEL ${ }^{\text {var }}$ sanctorum ] om. AMst ${ }^{\mathrm{var}}$; + doceo $51545^{8} 777_{888} \mathrm{AMst}^{\mathrm{PEL}}{ }^{\mathrm{A}}$

14:34 ${ }^{\dagger}$ Present: $51545^{861^{\dagger}} 75^{\dagger} 76^{\dagger} 77788889$ AMst PEL ${ }^{\mathrm{A}}$ TES SPE mulieres (78) ] + uestrae $617576777^{\text {gl }} 89$ AMst $^{\text {ed }}$ PEL $^{\text {var(ed) }}$ TES $^{\text {ed }}$, + uero AMst $^{\text {var }}$

in ecclesiis taceant $5^{176}$ ] in ecclesia taceant $5^{8} \mathrm{AMst}^{\mathrm{var}} \mathrm{TES}^{\mathrm{var}}$, taceant in ecclesia 67 PEL $^{\text {var }}$, taceant in ecclesiis PEL ${ }^{\text {var(ed) }}$

non enim $\langle 77\rangle]$ abs. TES

permittitur ] permittetur PEL ${ }^{\text {var }}$, abs. TES

eis loqui sed ] illis loqui sed 757689 AMst SPE, abs. TES

subditas esse $54(75)$ ] esse in silentio AMsted, subiectas esse in silentio $\mathrm{AMst}^{\mathrm{AD} *}$, subditas esse in silentio $\mathrm{AMst}^{\mathrm{R}}$, abs. TES

sicut et lex dicit ] sicut lex dicit $61 \mathrm{AMst}^{\mathrm{var}} \mathrm{PEL}^{\mathrm{var}} \mathrm{SPE}$, abs. TES

14:35 Present: $51545^{8} 61757677788889$ AMst PEL ${ }^{\mathrm{A}}$ TES SPE

si quid autem 54 ] si quid 61 PEL var, si quae autem quid $\mathrm{TES}^{\mathrm{ed}}$, si quis autem qui TES ${ }^{\mathrm{var}}$, si quid autem quae TES ${ }^{\mathrm{var}}$, si autem TES $\mathrm{S}^{\mathrm{var}}$

uolunt discere $\left(88^{*}\right)$ ] uolunt dicere $54^{*}$, discere uolunt 7576777889 AMst $\mathrm{TES}^{\text {ed }}$, dicere uoluit TES ${ }^{\mathrm{var}}$; + usque hic PEL var $\dagger$

domi ] domo uel domi 77

uiros suos interrogent ] suos uiros interrogent 7576 77, uiros interrogant $\mathrm{PEL}^{\text {var }}$, interrogent uiros suos $\mathrm{SPE}^{\mathrm{var}}$

turpe est enim ] turpe enim est 77 (78), abs. TES

mulieri 78 PEL $^{\mathrm{var}}$ ] mulieribus $61757^{6} 777^{\mathrm{gl}} 89 \mathrm{AMst}^{\mathrm{ed}} \mathrm{PEL}^{\mathrm{A}}$, mulieres $\mathrm{AMst}^{\mathrm{AD} *}, a b s$. TES

loqui in ecclesia ] in ecclesia loqui $5^{1} 5^{8} 61 \mathrm{AMst}^{\text {ed }} \mathrm{PEL}^{\text {var(ed), in ecclesiis }}$ loqui $75767789 \mathrm{AMst}^{\mathrm{var}}$, abs. TES; + doceo 61

14:36 Present: 51545861757677788889 AMst PEL $5^{\mathrm{A}}$ SPE

an a uobis $61^{\text {alt }} \mathrm{AMst}^{\mathrm{ADR}}$ ] aut a uobis $61 \mathrm{AMst}^{\text {ed }} \mathrm{PEL}^{\mathrm{var}(\mathrm{ed})}$

uerbum ] sermo 75767789 AMst $^{\mathrm{AD}} \mathrm{PEL}^{\text {var }}$

$\dagger$ 1Cor. 14:34-35. These verses appear after 14:40 in VL 61, 75, 76 and all manuscripts of AMst (but not the printed editions, including $\mathrm{AMst}^{\mathrm{R}}$ ).

$\dagger{ }_{1}$ Cor. 14:35. The addition usque hic in PEL ${ }^{\mathrm{var}}$ is likely to be a note rather than part of the biblical lemma. 
dei ] om. 61 PEL var

processit $\mathrm{AMst}^{\mathrm{AD}}$ ] profectum est $\mathrm{AMst}^{\mathrm{ed}}$

aut in uos solos peruenit ] aut in uos solos deuenit $6189 \mathrm{AMst}^{\mathrm{ed}} \mathrm{PEL}^{\mathrm{A}} \mathrm{SPE}^{\mathrm{ed}}$, aut in uobis solis deuenit $7576 \mathrm{AMst}^{\mathrm{AD}}$, an in uos deuenit solos 77 , in uos solos peruenitur $78^{*}$, in uos solos peruenit $78^{\mathrm{C}}$, aut in uos solus peruenit PEL var, aut in uos solus euenit SPEvar

14:37 Present: $51545^{861757677788889 \text { AMst PEL }}$ A $^{\mathrm{A}}$

si quis uidetur $\mathrm{AMst} \mathrm{AD}^{\mathrm{AD}}$ ] si quis existimatur $\mathrm{AMst}^{\mathrm{ed}}$, si quis exaestimatur AMst tvar $^{\text {at }}$

propheta esse aut spiritalis $5^{1} 5^{861}$ ] propheta esse uel spiritalis $\mathrm{AMst} \mathrm{AD}^{\mathrm{AD}}$ cognoscat ] agnoscat 54 , cognoscet AMst $^{\mathrm{R}}$

quae scribo uobis $\left.51545^{8} 767778 \mathrm{PEL}^{\mathrm{var}(\mathrm{ed})}\right]$ quae uobis scribo $61 \mathrm{PEL}^{\mathrm{var}}$, om. PELA

quia domini

sunt mandata $\mathrm{AMst}^{\mathrm{R}}$ ] sunt $75767789 \mathrm{AMst}^{\mathrm{ed}} \mathrm{PEL}^{\mathrm{var}}$, mandata sunt 78 , est mandatum $\mathrm{AMst}^{\mathrm{AD}}$

14:38 Present: 51545861757677788889 AMst PEL ${ }^{\mathrm{A}}$

si quis autem ] si autem quis 77 , si quis PEL var

ignorat PEL ${ }^{\text {ed }}$ ] ignorant 75 ; + haec PEL ${ }^{\mathrm{A}}$

ignorabitur ] ignoratur 757689

14:39 Present: $51545^{8} 61757677788889$ AMst PEL $^{\mathrm{A}}$

itaque fratres $\mathrm{AMst} \mathrm{t}^{\mathrm{AD}}$ ] propter quod fratres $\mathrm{AMst}^{\mathrm{ed}} ;+$ mei $5^{8}$

aemulamini prophetare $51545^{8} 6176777888 A M s t^{A D}$ ] aemulationem habete profetandi AMst ${ }^{\text {ed }}$

et loqui ] + in $7576,+$ autem 77

linguis

nolite prohibere 5488 ] ne prohibete 77 , non prohibeatis $\mathrm{AMst}^{\mathrm{AD}}$

14:40 ${ }^{\dagger}$ Present: $5^{1} 545861^{\dagger} 75^{\dagger} 76^{\dagger} 77788889 \mathrm{AMst}^{\dagger} \mathrm{PEL}^{\mathrm{A}}$

omnia autem AMst ${ }^{\text {var }}$ ] omnia enim $88 \mathrm{AMst}^{\text {ed }} \mathrm{PEL}^{\text {var }}$ + uestra $61 \mathrm{PEL}^{\text {var(ed) }}$ honeste 788889 ] honesta 61

et secundum ordinem fiant ] et secundum [5] fiant 54 *, fiant 6189 PEL $^{\text {var, }}$, et ordine fiant 75 , et ordinate fiant 76 ; + in uobis $51545^{8} 88^{*}$

$\dagger 1$ Cor. 14:40. Verses 34-35 appear after 14:40 in VL 61, 75, 76 and AMst. 
15:1 Present: $515458617576777884888925^{\dagger}{ }^{\dagger}$ AMst PEL ${ }^{\mathrm{A}}$ CAr

\pm fratres 84

notum autem uobis facio (78) ] notum uobis facio $54 * 84 \mathrm{AMst}^{\mathrm{R}}$, notum enim uobis facio 7576 , notesco autem uobis $777^{\mathrm{gl}}$, notum autem uobis facimus $25^{2}$, notum autem facio uobis AMst ${ }^{\mathrm{AD}}$

fratres ] om. 84; + quoniam $61 \mathrm{PEL}^{\text {var(ed) }}$, quia AMst ${ }^{\text {ed }}$

euangelium 545861 ] abs. CAr

quod praedicaui 515458617888 ] quod euangelizaui $75767789 \mathrm{AMst}^{\mathrm{AD}}$, abs. $84 \mathrm{CAr}$

uobis quod et accepistis in quo et statis 61 ] abs. $84 \mathrm{CAr}$

15:2 Present: $5^{1} 545^{8} 617576777^{8} 8889251$ AMst PEL $^{\mathrm{A}}$

per quod et $\left(25^{2}\right)$

saluamini ] salui efficimini 757689 AMst $^{\text {ed }}$, salui efficiemini AMst ${ }^{\mathrm{ADR}}$

qua ratione $58(251) \mathrm{AMst}^{\mathrm{AD}}$ ] quo sermone $\mathrm{AMst}^{\mathrm{ed}}$, quod et sermone $\mathrm{AMst}^{\mathrm{R}}$; + ante $6188^{*}$ PEL $^{\text {var(ed) }}$, + et 75

praedicauerim uobis 5154586188 ] euangelizaui uobis 7576 (77) $89 \mathrm{AMst}^{\mathrm{AD}}$, adnuntiaui uobis AMst ${ }^{\text {ed }}$

si AMst ${ }^{\mathrm{AD}}$ ] om. $617576778925^{2}$ AMst $^{\mathrm{ed}} \mathrm{PEL}^{\mathrm{var}}$, lac. 88*

tenetis $\mathrm{AMst}{ }^{\mathrm{AD}}$ ] retinetis 58 , debetis tenere $61758925^{2}$ AMst $^{\text {ed }}$ PEL $^{\text {var, }}$, debetis sustinere 76 , debetur tenere 77 , lac. $88^{*}$

nisi si $\left.\left\langle 54^{*}\right\rangle \mathrm{PEL}^{\mathrm{var}}\right]$ nisi $5^{1}$ 54 $^{\mathrm{C}} 5^{8} 617576777^{8} 88^{\mathrm{C}}$ 251AMst PEL ${ }^{\mathrm{A}}$, lac. $88^{*}$ frustra ] sine causa $75768925^{1} \mathrm{AMst}^{\mathrm{AD}}$, sine causa uel frustra 77 , forte sine causa AMst ${ }^{\text {ed }}$

credidistis $25^{2}$

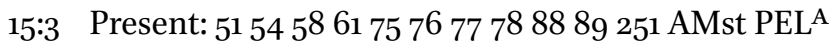

tradidi enim uobis in primis $\left(78^{*}\right)$

quod et accepi 61 ] sicut accepimus 75 76, om. 89 AMst $^{\text {ed }}$, quod accepi $\mathrm{AMst}^{\mathrm{AD}} \mathrm{PEL}^{\mathrm{var}}$

quoniam ] quia $757677788925^{2} \mathrm{AMst}^{\mathrm{AD}}$

christus ] iesus christus 58

mortuus est pro peccatis nostris secundum $\left(54^{*}\right)$

scripturas $\left(78^{\mathrm{C}}\right)$ ] lac. $78^{*}$, scripturam PEL var

$\dagger{ }_{1}$ Cor. 15 . There are three overlapping lections in VL 251, covering 15:1-19, 15:1-22 and 15:1228. 
15:4 Present: 51545861757677788889251 AMst PEL $^{\dagger}$ et quia sepultus est $\left(54^{*}\right)$ ] lac. $78^{*}$, et sepultus est PEL ${ }^{\mathrm{A}}$ et quia ] et 58 resurrexit ] surrexit $54^{*} 7576777889 \mathrm{AMst}^{\mathrm{var}} \mathrm{PEL}^{\mathrm{A}}$ tertia die 515461251 ] die tertia 75 76, tertio die AMst ${ }^{\mathrm{var}}$ secundum scripturas (89) ] om. $5^{1}$

15:5 Present: 5154586164757677788889 251 AMst PEL et quia uisus est 61 ] lac. 64 cephae et $51545^{8}\langle 64\rangle 76778825^{1}$ ] cephae AMst post haec $51545^{8}$ ] lac. 64 , postea $757^{6}$ AMst, postea uel post haec 77 , post 89 \pm illis $54^{*} 61757_{7} 7^{\text {gl }} 89$ AMst $^{\text {ed }} \mathrm{PEL}^{\mathrm{AB}}$, aliis $\mathrm{AMst}^{\mathrm{A}}$ undecim $25^{2}$ ] lac. 64

15:6 Present: $5^{1} 545^{8} 61757677788889$ 251 AMst PEL deinde uisus est 61 ] deinde apparuit 75767789 AMst plus quam ] amplius 7576 , plus uel plus quam amplius 77 , amplius quam 89 quingentis fratribus simul ex quibus 61 multi AMstrar ${ }^{\text {var }}$ plures 75767789 AMst $^{\text {ed }}$ manent usque adhuc $\left(88^{*}\right)$ quidam autem ] quid autem 75 , quidam $A M s t^{\mathrm{var}}$, om. $\mathrm{PEL}^{\mathrm{A}} ;+$ ex eis $5^{8}$ dormierunt ] om. PEL ${ }^{\mathrm{A}}$

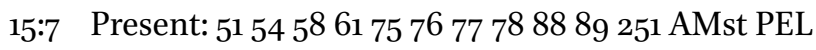
deinde uisus est 61 ] deinde uisus $54^{*}$, deinde apparuit $757^{6} 77^{\mathrm{C}} 89 \mathrm{AMst}$ iacobo ] petro $\mathrm{AMst}^{\mathrm{var}}$ deinde ] postmodum (75) 7677 89, postea AMst apostolis omnibus 251

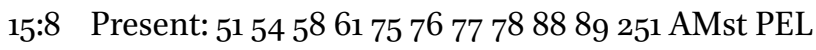
nouissime autem omnium ] nouissime autem 7576 tamquam $\left.77 \mathrm{AMst}^{\text {var }}\right]$ quasi AMst ${ }^{\text {ed }}$ abortiuo uisus est $54^{C} 6189251^{2}$ et mihi $51545^{8}$ ] mihi AMst ${ }^{\text {var }}$

$\dagger \quad 1$ Cor. 15:4. PEL ${ }^{\mathrm{B}}$ resumes in this verse. 
15:9 Present: $5^{1} 545^{8} 61757677788889$ 251 AMst PEL ego enim ] ego autem 61 sum minimus ] minimus sum $\mathrm{PEL}^{\mathrm{B}}$ apostolorum ] omnium apostolorum $251^{2} \mathrm{AMst}^{\text {ed }} \mathrm{PEL}$ var qui non sum dignus ] quia non sum dignus $\mathrm{AMst}^{\mathrm{var}}$ uocari ] uocare 251 apostolus $251^{2}$ ] apostolos $251^{1}$ quoniam $\mathrm{AMst}^{\mathrm{var}}$ ] propter quod 77, quia AMst ${ }^{\mathrm{ed}}$ persecutus sum ecclesiam dei ${ }_{51} 6189$ ] persecutus ecclesiam dei $251^{1 *}$

15:10 Present: 51545861757677788889251 AMst PEL gratia autem dei $5825^{1}$ ] gratia enim dei $25^{2} \mathrm{PEL}^{\mathrm{var}}$, gratia dei AMst ${ }^{\mathrm{A} *}$ sum id AMstrar ] sum $757688^{*}$ AMsted $^{\text {ed }}$ LL $^{\text {var }}$ quod sum et gratia eius 58251 in me uacua non fuit ] in me paupera non fuit 757689 , in me pauper non fuit 77 , non fuit in me uacua $251^{2}$, pauper in me non fuit AMsted, pauper non fuit in me AMstrar, in me egens non fuit $\mathrm{AMst}^{\mathrm{AD}}$, egena in me non fuit $\mathrm{AMst}^{\mathrm{R}}$, in me egena non fuit $\mathrm{PEL}^{\mathrm{B}}$

sed abundantius 51545861 (88) $251 A M{ }^{A D} t^{A D}$ sed plus 89 AMsted $^{\text {ed }}$ illis omnibus ] omnibus illis 515489 , quam illi AMst ${ }^{\mathrm{AD}}$, omnibus AMst ${ }^{\mathrm{var}}$ laboraui ] laborauit $\mathrm{AMst}^{\mathrm{D}}$; + et obtinui $61 \mathrm{PEL}^{\mathrm{B}}$, + et tenui $\mathrm{PEL}^{\text {var }}$ non ego autem ] non autem ego $5458 \quad 7^{8} \quad 25^{2} \mathrm{PEL}^{\mathrm{var}}$, non ego ex me PEL $^{\mathrm{B}}$

sed gratia dei mecum $5825^{2}$ ] sed domini gratia mecum AMst' ${ }^{\mathrm{var}}$, sed gratia mecum PEL var

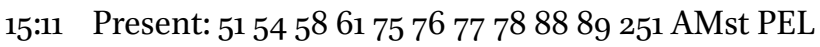
siue enim ] siue autem 75767778 89, siue igitur AMsted, siue ergo $\mathrm{AMst}^{\mathrm{var}}$ ego siue illi ] ego sicut illis AMst ${ }^{\text {var }}$ sic ] ita 757689 , sic uel ita 77 praedicamus $515861767788^{*} P E L^{A}$ ] praedicauimus $5488^{C} 25^{2} \mathrm{PEL}^{\mathrm{var}}$ et sic $\mathrm{AMst}^{\mathrm{var}}$ ] et ita $757689 \mathrm{AMst}^{\text {ed, }}$, et sic uel ita 77 credidistis

15:12 Present: $515458617576777888892^{251^{\dagger}}$ AMst PEL si autem christus ] fratres si autem christus $25^{3}$ praedicatur 515458617788 ] praedicatus 7576

$\dagger \quad$ 1Cor. 15:12. A third lection begins in VL 251 in this verse. 
quod resurrexit a mortuis $54 \mathrm{AMst}^{\mathrm{AD}}$ ] ex mortuis quod resurrexit 7576 , a uel ex mortuis quod resurrexit 77 , quod a mortuis resurrexerit 89 , quod surrexit a mortuis $251^{1} \mathrm{PEL}^{\mathrm{B}}$, quod ex mortuis resurrexit AMst ${ }^{\mathrm{ed}}$, quod ex mortuis resurrexerit $\mathrm{AMst}^{\mathrm{var}}$

quomodo ] om. PEL var

quidam dicunt ] dicunt quidam $7677 \mathrm{AMst}^{\mathrm{ed}}$, quidem dicunt $\mathrm{AMst}^{\mathrm{AD}}$, dicant quidam AMst ${ }^{\mathrm{var}}$

in uobis $\mathrm{AMst}^{\mathrm{AD}}$ ] inter uos AMsted

quoniam ] quod 517577, om. 76 , quia 89 , quomodo AMst ${ }^{\text {var }}$

resurrectio mortuorum non est $\langle 51\rangle 54]$ om. 76

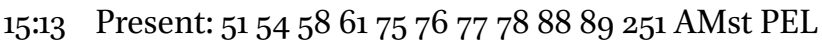

si autem $\mathrm{AMst}^{\mathrm{AD}}$ ] quod si 546189 , nam si 75 , quod 76, si ergo AMsted

resurrectio mortuorum 54

non est ] est 61 , non es 78

neque ] nec 7576

christus resurrexit 54 ] christus surrexit 61 PEL $^{\text {var }}$

15:14 Present: 5154586164757677788889 251 AMst PEL

si autem christus non ] om. $5^{8} 7576$, lac. 64

resurrexit 54 ] om. $5^{8} 7576$, surrexit $61 \mathrm{AMst}^{\mathrm{var}}$, lac. 64

inanis est ergo ] inanis est $586175768925^{2}{ }^{2} \mathrm{AMst}^{\text {ed }} \mathrm{PEL}^{\mathrm{B}}$, lac. 64, inanis igitur et 77 , inanis ergo est $\mathrm{AMst} \mathrm{AD}^{\mathrm{AD}}$

praedicatio nostra $5154586176778825^{1}$ ] lac. 64

inanis ] uacua $547589 \mathrm{AMst}^{\text {ed }}$, uane $\mathrm{AMst}^{\mathrm{var}}$

est et $\mathrm{AMst}^{\mathrm{var}}$ ] est $757689 \mathrm{AMst}^{\text {ed }}$, et 77 , est ergo et PEL ${ }^{\mathrm{A}}$

fides uestra ] fides nostra 61

15:15 Present: $51545^{8} 6164757677788889251$ AMst PEL

inuenimur ] inuenimus (54)

autem et falsi $\left.\left(251^{1 *}\right)\right]$ etiam falsi $757689 \mathrm{AMst}^{\mathrm{AD}}$, ergo et falsi $251^{3}$, et falsi autem $\mathrm{AMst}^{\mathrm{var}}$, autem eam falsi PEL ${ }^{\mathrm{A}}$, et falsi $\mathrm{PEL}^{\mathrm{B}}$

testes dei $25^{2}$ ] testes $88^{*}$

quoniam ] quia 6489 AMst, quod 7576 , quia uel quoniam 77

testimonium diximus ] testati sumus 77

aduersus deum ] aduersum deum 89

quod ] quia 64 , quem 89

suscitauerit ] suscitabit 64, suscitauit $\mathrm{AMst}^{\mathrm{A}} \mathrm{PEL}^{\mathrm{B}}$

christum quem non

suscitauit ] resuscitauit 61, suscitabit 64 
si mortui non resurgunt $54 \mathrm{AMst}^{\mathrm{R}}$ ] om. $5^{1} 5^{8} 616475768889251 \mathrm{AMst}^{\mathrm{ed}}$ $\mathrm{PEL}^{\mathrm{B}}$, siquidem igitur mortui non resurgunt 77 , quippe si mortui non resurgunt $\mathrm{AMst}^{\mathrm{AD}}$

15:16 Present: $51545^{8} 6164757^{6} 777_{88} 88$ 89 251 AMst PEL

nam si $\mathrm{AMst}^{\mathrm{R}}$ ] om. $54^{*}$, si $5825^{3} \mathrm{PEL}^{\text {var, }}$, si enim $6175767789 \mathrm{AMst}^{\mathrm{ed}}$, si ergo 64, si autem $\mathrm{AMst}^{\mathrm{AD}}$, si quidem $\mathrm{PEL}^{\mathrm{B}}$

mortui non resurgunt $5_{4}^{C} \mathrm{AMst}^{\mathrm{ADR}}$ ] om. $54^{*}$, mortui non resurgent AMsted

neque christus ] nec christus $\mathrm{AMst}^{\mathrm{AD}}$

resurrexit 54 ] surrexit $7576 \mathrm{AMst}^{\mathrm{var}}$

15:17 Present: 5154586164757677788889 251 AMst PEL quod si ] si autem $647778 \mathrm{AMst}^{\mathrm{AD}}$

christus non resurrexit 54 ] christus non surrexit $58^{*}$ uana est $\mathrm{AMst}^{\mathrm{ADR}}$ ] om. AMsted; + ergo PEL var

fides uestra $\mathrm{AMst}^{\mathrm{ADR}}$ ] fides nostra 58 , om. $\mathrm{AMst}^{\mathrm{ed}}$; + quid $757^{6 \mathrm{C} *}$, quia 89

adhuc ] om. $76^{*}$, a $[$ dhuc $] 7^{\text {C** }}$

enim estis $\mathrm{AMst}^{\mathrm{R}}$ ] estis $64757789 \mathrm{AMst}^{\mathrm{ed}}$, om. 76

in peccatis uestris ] om. $76^{*}$, in peccatis $(77)$

15:18 Present: $51545^{8} 6164757677788889$ 251 AMst PEL ergo $\mathrm{AMst}^{\mathrm{ADR}}$ ] om. AMsted

et qui ] qui $89 \mathrm{PEL}^{\mathrm{A}}$

dormierunt ] dormierant $\mathrm{PEL}^{\mathrm{B}}$; + uel dormientes 77

in christo perierunt ] in christo non perierunt $\mathrm{AMst}^{\mathrm{var}}$

15:19 Present: 5154586164757677788889 251 AMst PEL

si ] et si 61

in hac uita ] in uita hac 757677

tantum in christo sperantes sumus AMstrar ] tantum sperantes sumus in christo $54 \mathrm{AMst}^{\mathrm{ed}}$, in christo sperantes sumus tantum 6475767789 miserabiliores

sumus omnibus hominibus ] omnibus hominibus sumus $\left(77^{*}\right)^{\mathrm{C}}$

15:20 Present: 5154586164757677788889251 AMst PEL SPM nunc autem $\mathrm{AMst}^{\mathrm{ADR}}$ ] si autem $757689 \mathrm{PEL}^{\mathrm{B}}$, si enim $\mathrm{AMst}^{\mathrm{ed}}$ christus resurrexit $54^{*}$ ] surrexit christus 89 , christus surrexit $251^{1} \mathrm{PEL}^{\text {var }}$ SPM, resurrexit christus PEL var 
a mortuis (77) $\mathrm{SPM}^{\mathrm{var}}$ ] ex mortuis $\mathrm{SPM}^{\mathrm{ed}}$

primitiae dormientium $5154 \quad 5^{8} \quad 2^{11^{1}}$ ] initium dormientium 757677 $\mathrm{AMst}^{\mathrm{ed}} \mathrm{SPM}^{\mathrm{var}}$, principium dormientium $\mathrm{AMst}^{\mathrm{AD}}$, incohatio dormientium SPM ${ }^{\text {ed }}$

15:21 Present: 5154586164757677788889251 AMst PEL CAr SPM quoniam enim ] quoniam quidem $5^{1} 5^{\mathrm{C}} 5^{8} 6475768889$ AMst PEL var CAr $\mathrm{SPM}^{\mathrm{var}}$, quoniam $61 \mathrm{PEL}^{\mathrm{AB}}$, quoniam quidem enim 77 , nam quia $\mathrm{SPM}^{\mathrm{ed}}$; + est $58,+$ sicut 61 PEL $^{B}$

per hominem mors (64) ] + ita 61 et per hominem resurrectio mortuorum $54\left(88^{*}\right)$

15:22 Present: $5^{1} 545^{8} 6164757677788889$ 251 AMst PEL SPM et sicut ] sicut enim 616475767789 AMst $^{\text {ed }}$ PEL $^{B}$ SPM, si enim AMst ${ }^{\text {var }}$ in adam omnes moriuntur ita et $\mathrm{SPM}^{\mathrm{var}}$ ] sic et 64 , ita $88 \mathrm{SPM}^{\mathrm{ed}}$ in christo omnes $25^{2}$ uiuificabuntur ] uiuificantur $6189 \mathrm{PEL}^{\text {var }}$

15:23 Present: $5^{1} 545^{8} 6164757677788889$ 251 AMst PEL sPM unusquisque autem ] unusquisque enim AMstrar in suo ordine primitiae $5^{1} 545^{8} \mathrm{AMst}^{\mathrm{ADR}}$ ] initium $64757689 \mathrm{AMst}^{\mathrm{ed}} \mathrm{SPM}$, initium uel primitiae 77 ; + uero 51 christus deinde ] christus de[2]de $88^{*}$

hii qui sunt $5^{1} 75767778888925^{1}$ AMst $^{\text {ed }}$ ] qui sunt $\mathrm{AMst}^{\mathrm{R}}$ christi ] in christo AMst ${ }^{\mathrm{AD} *}$; + qui $5^{1} 54^{\mathrm{C}} 5^{8} 61778889251 \mathrm{AMst}^{\mathrm{ADR}} \mathrm{PEL}^{\mathrm{B}}$ SPM

in aduentu eius $\left.A M s t^{\text {var }} S \mathrm{PM}^{\mathrm{var}}\right]$ in aduentum eius $5^{1} 54^{\mathrm{C}} 61 \mathrm{AMst}^{\mathrm{ed}} \mathrm{SPM}{ }^{\mathrm{ed}}$, in praesentia eius 64 \pm crediderunt $5^{1} 545^{8^{C}} 61888925^{1}$ AMst $^{\mathrm{ADR}} \mathrm{PEL}^{\mathrm{B}} \mathrm{SPM}$, crediderint $58^{*}$, sperauerunt uel sperantes 77 , sperantes $7^{\mathrm{gl}}$

15:24 Present: $5^{1} 545^{8} 6164757677788889$ 251 AMst PEL sPM deinde finis cum ] abs. SPM; + autem 54 * tradiderit regnum deo ] abs. SPM et patri AMst ${ }^{\text {var }}$ ] patri $5^{8} \mathrm{AMst}^{\mathrm{ed}}$, abs. SPM; + et $54^{\mathrm{C}} 5^{8}$ cum euacuauerit (78) $\mathrm{AMst}^{\mathrm{AD}}$ ] cum destituerit $\mathrm{AMst}^{\mathrm{ed}}$, abs. SPM omnem principatum $\left(78^{*}\right)$ ] abs. SPM 
et potestatem ] et omnem potestatem 6475767789 AMst, abs. SPM et uirtutem $\mathrm{AMst}^{\mathrm{var}}$ ] om. AMst ${ }^{\mathrm{ed}}$, et omnem uirtutem $\mathrm{AMst}^{\mathrm{var}}$, abs. SPM \pm et dominationem $58 \mathrm{AMst}^{\mathrm{AD}}$, et omnem dominationem $\mathrm{AMst}^{\mathrm{ed}}$

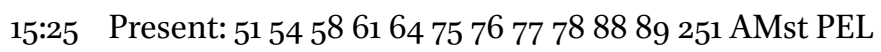

oportet autem ] oportet enim 61647576777889 AMst PEL ${ }^{\text {var, }}$, oportet PEL $^{\mathrm{B}}$

illum ] eum $\mathrm{AMst}^{\mathrm{AD}}$

regnare donec ponat omnes inimicos (251) ] + suos 616478, + eius 77

sub pedibus 54$]+$ uel pedes 77

eius ] suis 6164 , om. 77

$\pm^{\dagger}$ omnia enim subiecit sub pedibus eius 54 (61) 757689 AMst $^{\text {ed }}$ PEL $^{\text {var }}$, omnia enim subiecisti sub pedes eius $\mathrm{AMst}^{\mathrm{var}}$, mors omnia enim subiecit sub pedibus eius AMst $^{\mathrm{R}}$

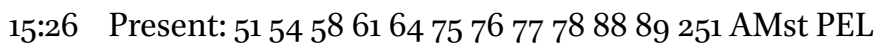

nouissima AMst ${ }^{\text {var }}$ ] nouissime 6164 AMst $^{\text {ed }}$, nouissimos 7576

autem $\mathrm{AMst}^{\mathrm{R}}$ ] om. $6475767789 \mathrm{AMst}^{\mathrm{ed}}$; + omnium $5^{8}$

inimica AMst ${ }^{\text {var }}$ ] inimicos 7576, om. AMst ${ }^{\text {ed }}$

destruetur mors 61 ] destruitur mors 757689 , euacuabitur mors $\mathrm{AMst}^{\mathrm{AD}}$, destruatur mors AMst ${ }^{\text {var }}$

15:27 Present: $51545^{8} 6164757677788889$ 251 AMst PEL

omnia enim subiecit sub pedibus eius PEL ${ }^{\text {var }}$ ] om. (cf. 15:25) 5461757689

AMst PEL ${ }^{A B}$, omnia enim sub pedibus eius 58

cum autem ] cum uero $\mathrm{AMst}^{\mathrm{R}}$

dicat ] dixer[it] 64, dicit 78; + quia $6478,+$ quoniam uel quia 77

omnia subiecta

sunt ] esse PEL var; + ei $5^{1} 545^{8} 61777888251$ AMst PEL $^{\mathrm{B}}$

sine dubio ] manifestum est quia $\langle 64\rangle \mathrm{PEL}^{\text {var }}$, om. $75767789 \mathrm{AMst}^{\text {ed }}$, profecto $\mathrm{AMst}^{\mathrm{AD}}$, nimirum $\mathrm{AMst}^{\mathrm{R}}$, manifestum quia PEL var

\pm nihil praetermisit non subiectum ei $54^{c}$, nihil domini sit non subiectum ei 58

praeter eum 51545861767788 ] praeter eum uel quem 77 , praeter illum $\mathrm{AMst}^{\mathrm{var}}$

$\dagger{ }_{1}$ Cor. 15:25. The addition at the end of the verse comes from 1 Cor. 15:27; different manuscripts of PEL have it at each place. 
qui subiecit ] qui sine dubio subiecit 77

ei omnia $\mathrm{AMst}^{\mathrm{var}}$ ] illi omnia $64 \mathrm{AMst}^{\mathrm{ed}}$, sibi omnia PEL ${ }^{\mathrm{B}}$

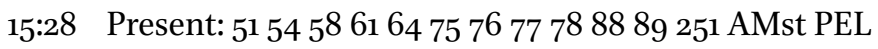

cum autem ] nam cum 547576 , cum uero 89 AMst $^{\mathrm{AD}}$

subiecta fuerint illi omnia $\mathrm{AMst} \mathrm{AD}^{\mathrm{AD}}$ ] subiecta illi fuerint omnia $5^{1} 545^{8}$

$7576 \mathrm{AMst}^{\mathrm{ed}} \mathrm{PEL}^{\mathrm{B}}$, omnia ei subiecta fuerint 64 , subiecta illi omnia

$77^{*}$, subiecta illi omnia fuerint $77^{\mathrm{C}}$, subiecta fuerint ei omnia 78 , omnia

subiecta illi fuerint $\mathrm{AMst}^{\mathrm{var}}$

tunc ] + et 616478 AMsted $^{\text {ed }}$ PEL $^{\mathrm{AB}}$

ipse filius ] ipse AMsted, ille filius AMstrar

subiectus erit ] subditus erit 7576 , erit subiectus uel subicietur 77 , subicietur

$\mathrm{AMst}^{\mathrm{R}}$ PEL $^{\text {var }}$, subiecitur PEL ${ }^{\text {var }}$, subicit PEL ${ }^{\text {var }}$

illi qui $\mathrm{AMst}^{\mathrm{R}}$ ] ei qui $6475767889 \mathrm{AMst}^{\mathrm{ed}} \mathrm{PEL}^{\text {var }}$, qui $\mathrm{PEL}^{\text {var }}$

sibi subiecit $A M s^{\text {var }}$ ] subiecit sibi 61, illi subiecit 64 AMst $^{\text {ed }}$, si subdidit 75 ,

sibi subdidit $7689 \mathrm{AMst}^{\mathrm{AD}}$, subiecit ei 77 , ei subiecit $\mathrm{AMst}^{\mathrm{R}}$

omnia ut ] om. $89^{*}$

sit deus ] deus sit $757^{689^{\mathrm{C}}}$, om. $89^{*}$, sit dominus AMstvar

omnia in omnibus

15:29 Present: 5154586164757677788889 AMst PEL

alioquin quid $\left(64^{*}\right)$ ] sin alias quid 89

facient ] faciunt 64 , faciet 7576

qui baptizantur 5461 ] om. 58 , baptizati uel qui baptizantur 77

pro mortuis si ] om. $5^{8}$

omnino mortui non ] om. 58 , omnium mortui non AMstvar

resurgunt $54 \mathrm{AMst}^{\mathrm{ADR}}$ ] om. 58 , resurgent $77 \mathrm{AMst}^{\mathrm{ed}}$, surgunt $\mathrm{PEL}^{\text {var }}$, praesurgunt $\mathrm{PEL}^{\mathrm{var}}$

ut quid $54 \mathrm{AMst}^{\mathrm{R}}$ ] qui $58 \mathrm{AMst}^{\mathrm{var}}$, quid $6489 \mathrm{AMst}^{\mathrm{ed}}$, qui quid 7576

et ] om. $5^{1} 5^{8} 757^{6} 78$, etiam 61

baptizantur pro illis $545861\left(88^{*}\right)$

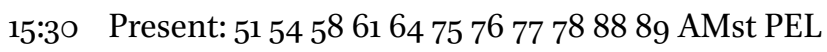

ut quid $54 \mathrm{AMst}^{\mathrm{ADR}}$ ] quid $647576 \mathrm{AMst}^{\mathrm{ed}} \mathrm{PEL}^{\mathrm{B}}$

et nos ] nos 51

periclitamur omni hora $54\left(88^{*}\right)$ ] periclitamur omnia hora 75

15:31 Present: 5154586164757677788889 251 AMst PEL

cotidie morior 57 (54) $5861647589^{*}$ ] fratres cotidie morior 251

per ] propter $515^{8} 61 \mathrm{PEL}^{\mathrm{B}}$, pro $6478 \mathrm{AMst}$ 
uestram gloriam ] gloriam uestram $5^{8}$ 61, uestra gloria $78 \mathrm{AMst}^{\mathrm{var}}$, gloria uestra AMst ${ }^{\text {ed }}$

fratres quam habeo in $\mathrm{AMst} \mathrm{AD}^{\mathrm{AD}}$ ] quam habeo in $757677\left(78^{*}\right)^{\mathrm{C}} 8925^{1} \mathrm{AMst}^{\mathrm{ed}}$ PEL

christo iesu domino nostro AMst $\left.{ }^{\mathrm{ADR}} \mathrm{PEL}^{\text {var }}\right]$ domino $757689 \mathrm{AMst}^{\text {ed }}$ PEL ${ }^{A B}$

15:32 Present: $5^{1} 545^{8} 6164757677788889$ 251 AMst PEL

si secundum hominem

ad bestias ] bestiis 64757689 , cum bestiis $\mathrm{AMst}{ }^{\mathrm{AD}}$

pugnaui ephesi $6178 A M s t^{e d}$ ] ephesi pugnaui 64 , pugnaui in epheso uel ephesi 77 , pugnaui epheso $A M s t^{A}$

quid mihi prodest si mortui non $51545^{8}$

resurgunt $54 \mathrm{AMst}^{\mathrm{ADR}}$ ] resurgent $\mathrm{AMst}^{\mathrm{ed}}$

manducemus et bibamus cras enim

moriemur ] moriamur $77^{*} \mathrm{PEL}^{\text {var }}$

15:33 Present: 5154586164757677788889 251 AMst PEL TES SPM SPE

nolite ] abs. TES, et nolite SPEvar

seduci ] errare 77, seducere PEL ${ }^{\text {var }}$, abs. TES

corrumpunt 64 AMst ] + enim $51 \mathrm{SPM}^{\mathrm{var}}$

mores bonos $\left(25^{*}\right)$ TES $\left.^{\text {var }}\right]$ bonos mores 51 PEL $^{\text {var }}$, ingenia bona TES ${ }^{\text {ed }}$; et TES var $^{\text {var }}$

conloquia mala 51545861767778 TE S $^{\text {var }}$ ] conscientia mala $P E L^{v a r}$, confabulationes pessimae TES ${ }^{\text {ed }}$

15:34 Present: $51545^{8} 6164757677788889$ 251 AMst PEL SPM SPE

euigilate $\left.78 \mathrm{PEL}^{\mathrm{var}}\right]$ sobrii estote $61647576777^{\mathrm{gl}} 89 \mathrm{AMst}^{\mathrm{AD}} \mathrm{PEL}^{\mathrm{AB}} \mathrm{SPM}$, uigilate $\mathrm{AMst}^{\mathrm{ed}}$

iuste PEL var ] iusti $51545^{8} 6475767888251 \mathrm{PEL}^{\mathrm{AB}} \mathrm{SPM}^{\mathrm{var}} \mathrm{SPE}^{\mathrm{var}}$, et iusti 61 $\mathrm{sPM}^{\text {ed }}$, iusti estote $\mathrm{AMst}^{\mathrm{ed}}, \mathrm{om}$. AMst $\mathrm{AD}^{\mathrm{AD}}$

et nolite peccare ignorantiam enim $58\langle 64\rangle$

dei quidam habent $(75)]$ dei [hab] ent quidam 64, dei quidam dei habent PEL ${ }^{\text {var }}$, quidam dei habent SPM ${ }^{\text {var }}$

ad reuerentiam $\left.58(61)\left(77^{*}\right)\right]$ ad uerecundiam $A M s t^{\mathrm{R}} \mathrm{SPM}$

uobis loquor $\langle 64\rangle$ ] uobis dico $61777^{8} \mathrm{AMst}^{\mathrm{AD}}$, dico uobis SPM

15:35 Present: $5^{1} 545^{8} 6164757677788889$ 251 AMst PEL SPM

sed dicet $\left.A M s t^{\mathrm{R}}\right]$ sed dicit $516475767778\left(88^{*}\right)^{\mathrm{C}} 89 \mathrm{AMst}^{\mathrm{ed}} \mathrm{PEL}^{\mathrm{var}}$ SPM aliquis quomodo $\left.\mathrm{AMst}^{\mathrm{AD} *}\right]$ aliquis quemadmodum $\mathrm{AMst}^{\mathrm{ed}}$ 
resurgunt mortui $54^{*}$ ] resurgent mortui $54^{\mathrm{C}} 777888^{*} \mathrm{AMst}^{\text {ed }} \mathrm{PEL}^{\mathrm{A}}$, mortu[i] resurgunt 64, surgunt mortui $\mathrm{AMst}^{\mathrm{AD} *}$

quali ] quo $64757689 \mathrm{AMst}^{\mathrm{ed}} \mathrm{SPM}^{\mathrm{ed}}$, quoue $\mathrm{AMst}^{\mathrm{R}}$, quomodo $\mathrm{SPM}^{\mathrm{var}}$ autem corpore ] corpore $\mathrm{AMst}^{\mathrm{R}}$, autem in corpore $\mathrm{SPM}^{\mathrm{var}}$ ueniunt AMst ${ }^{\text {var }}$ ] uenient $5154^{\mathrm{C}} 7888 \mathrm{AMst}^{\mathrm{ed}}$, uen[...] 64

15:36 Present: $51545^{8} 6164757677788889$ 251 AMst PEL TES SPM

insipiens $\left.\mathrm{AMst}^{\mathrm{AD}} \mathrm{TES}^{\mathrm{var}}\right]$ stulte $64 \mathrm{AMst}^{\text {ed }} \mathrm{TES}^{\mathrm{ed}} \mathrm{SPM}^{\mathrm{var}}$

tu quod seminas ] tu qui seminas TES ${ }^{\text {var }}$

non uiuificatur $\langle 64\rangle]$ numquid uiuificatur TES ${ }^{\text {var }}$

nisi prius $\left.\mathrm{AMst}^{\mathrm{ADR}} \mathrm{TES}^{\mathrm{var}}\right]$ nisi $64 \mathrm{AMst}^{\text {ed }} \mathrm{TES}^{\mathrm{ed}} \mathrm{SPM}$, si non TES $\mathrm{S}^{\text {ed }}$

moriatur TES ${ }^{\text {var }}$ ] mortuum fuerit $\mathrm{AMst}^{\mathrm{AD}} \mathrm{TES}^{\text {ed }}$

15:37 Present: $5^{1} 545^{8} 6164757677788889$ 251 AMst PEL sPM

et quod seminas ] [4] $\mathrm{t}$ [3] quod seminas $54^{*}$, et quid seminas $\mathrm{AMst}^{\mathrm{R}}$

non corpus quod ] non ut $\mathrm{SPM}^{\mathrm{var}}$

futurum est $78 \mathrm{SPM}^{\mathrm{var}}$ ] nascetur $757^{6} 77$, nasciturum est $78^{\mathrm{gl}}$, futurum nascetur 89 , nascitur SPM ${ }^{\text {ed }}$

seminas sed nudum granum $\langle 64\rangle$

ut puta ] fere 64, utpote $77, \mathrm{om}$. $\mathrm{AMst}^{\mathrm{AD}} \mathrm{PEL}^{\mathrm{A}}$

tritici aut alicuius 64 ] om. $\mathrm{AMst}^{\mathrm{AD}} \mathrm{PEL}^{\mathrm{A}}$

ceterorum 6178 ] om. AMst ${ }^{\mathrm{AD}} \mathrm{PEL}^{\mathrm{A}}$, seminum sPM ${ }^{\mathrm{var}}$; + seminum 54

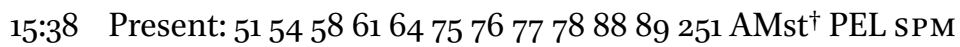

deus autem

dat illi corpus ] dat corpus 61, illi dat corpus 64757677 AMst $^{\text {ed }}$ PEL $^{\mathrm{B}}$ SPM sicut 88 ] quomodo $64 \mathrm{SPM}^{\text {var }}$, prout $757688^{\text {alt }} 89 \mathrm{AMst}^{\mathrm{ed}} \mathrm{SPM}^{\mathrm{ed}}$, sicut uel prout 77

uoluit $88^{*}$ ] uult $5154^{\mathrm{C}} 88^{\mathrm{C}} 88^{\text {alt }} 89 \mathrm{AMst}^{\mathrm{R}} \mathrm{SPM}^{\mathrm{ed}}$, uoluerit $64 \mathrm{SPM}^{\mathrm{var}}$ et unicuique 54 ] unicuique 89

seminum proprium corpus $(75)$ ] homini proprium corpus $54^{*}$

15:39 Present: $5^{1} 545^{8} 6164757677788889$ 251 AMst PEL CAr SPM non omnis caro eadem caro $\left(88^{*}\right)$ ] non omnis caro eadem 77 sed AMst ${ }^{\mathrm{ADR}}$ ] om. $64 \mathrm{AMst}^{\mathrm{ed}} \mathrm{PEL}^{\mathrm{B}}$, abs. CAr alia ] abs. CAr; + quidem 58616475767789 AMst PEL ${ }^{\mathrm{B}}$ SPM hominum AMst ${ }^{\mathrm{AD}} \mathrm{SPM}^{\mathrm{var}}$ ] hominis $757689 \mathrm{AMst}^{\mathrm{ed}} \mathrm{PEL}^{\mathrm{B}} \mathrm{SPM}^{\mathrm{ed}}$, abs. CAr

$\dagger{ }_{1}$ Cor. 15:38. AMst ${ }^{\mathrm{AD}}$ omit the whole of this verse. 
alia ] abs. CAr

\pm autem 6175767789 AMst $^{\text {ed }} \mathrm{PEL}^{\mathrm{B}}$, autem caro 64, uero $\mathrm{AMst}^{\mathrm{R}}$

pecorum $\left.5_{4}^{c} 5^{8} \mathrm{AMst}^{\mathrm{AD}} \mathrm{SPM}^{\mathrm{var}}\right]$ peccatorum $54^{*}$, pecoris $757689 \mathrm{AMst}^{\mathrm{ed}}$ PEL $^{B}$ SPM $^{\text {ed }}$, abs. CAr

alia ] om. 89, abs. CAr

caro sPM var ] om. $5^{1} 648889$ SPM $^{\text {ed }}$, uero $7778 \mathrm{AMst}^{\mathrm{var}}$, abs. CAr

uolucrum $61\langle 64\rangle \mathrm{SPM}^{\mathrm{var}}$ ] piscium 77 78, om. 89, uolatilium AMst SPM ${ }^{\mathrm{ed}}, a b s$. CAr

alia autem $A M s t^{A D}$ ] alia $6478 \mathrm{PEL}^{\text {var }} \mathrm{SPM}^{\mathrm{var}}$, alia caro $77 \mathrm{AMst}^{\mathrm{ed}}$, alia aut 88, abs. CAr

piscium ] uolucrum 77 78, abs. CAr

15:40 Present: $5^{1} 545^{8} 6164757677788889$ 251 AMst PEL sPM

et corpora ] corpora $\mathrm{SPM}^{\mathrm{var}}$

caelestia et $51545^{8} 767788$ ] cae[...] 64, caelestium et SPM $^{\text {var }}$

corpora $\langle 64\rangle \mathrm{AMst}^{\mathrm{ADR}}$ ] om. $7789 \mathrm{AMst}^{\mathrm{ed}}$

terrestria ] terrestrium SPM ${ }^{\mathrm{var}}$

sed ] sed et 251, si AMst'var, om. PEL ${ }^{\mathrm{B}}$ SPM, et PEL var

alia quidem ] alia $64 ;+$ aut 77

caelestium ${ }_{54}{ }^{c} 5^{8}>67788$ ] terrestrium 51 , caelestia 54 *

gloria ] + est 61

alia autem ] et alia $64 \mathrm{SPM}{ }^{\mathrm{var}}$, alia autem aut 77, alia PEL var

terrestrium ] terrestria 75

15:41 Present: $51545^{8} 6164757677788889251$ AMst PEL TES SPM

alia ] abs. TEs; + autem 77

claritas solis $\mathrm{SPM}^{\mathrm{var}}$ ] gloria solis $64757689 \mathrm{AMst}^{\mathrm{AD}} \mathrm{SPM}^{\mathrm{ed}}$, claritas uel gloria solis $77, a b s$. TES

alia ] et alia $5475^{*} 7689 \mathrm{SPM}^{\mathrm{var}}$, abs. TES

claritas lunae $515458767788 \mathrm{sPM}^{\mathrm{var}}$ ] gloria lunae $64757689 \mathrm{AMst}^{\mathrm{AD}} \mathrm{SPM}^{\mathrm{ed}}$, om. AMst ${ }^{\mathrm{R}}$, abs. TES

et alia ] alia (61) AMst ${ }^{\text {var } P E L}{ }^{\text {var }}$, abs. TES

claritas $\mathrm{SPM}^{\mathrm{var}}$ ] gloria $64757689 \mathrm{AMst}^{\mathrm{var}} \mathrm{SPM}^{\mathrm{ed}}$, om. AMst ${ }^{\mathrm{AD}}$, abs. TES

stellarum ] abs. TES

stella enim ] stella autem $6178 \mathrm{AMst}^{\mathrm{AD}}$, stella TES

ab stella PEL var ] stellae 757689 TES $^{\text {var }}$, stellae uel ab stella 77 , a stella PEL ${ }^{A B}$ $S P M^{\text {var }}$

differt 617588 ] defert TES ${ }^{\text {var }}$

in ] a AMst ${ }^{\text {var }}$

claritate ] gloria $64 \mathrm{AMst}^{\mathrm{AD}}$, caritate 75, om. 76 ; + sua $\mathrm{TES}^{\mathrm{var}}$ 


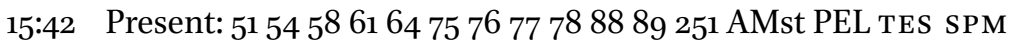
sic SPM ${ }^{\text {var }}$ ] ita 6175767789 AMst PEL $^{\text {B }}$ TES $^{\text {var }}$ SPM $^{\text {ed }}$; + erit AMsted et ] om. AMst ${ }^{\mathrm{var}}$

resurrectio mortuorum $54(77)$ ] resurrectio TE Svar $^{\mathrm{va}}$ seminatur 251 ] + corpus TES $S^{\text {ed }}$

in corruptione $5861 \mathrm{AMst}^{\mathrm{D*}}$ ] in corruptionem $757689 \mathrm{PEL}^{\text {var }} \mathrm{TES}^{\mathrm{var}}$, in corruptela $\mathrm{AMst}^{\mathrm{ed}}$, in correptionem $\mathrm{AMst}^{\mathrm{A}}$, in corruptelam $\mathrm{AMst}^{\mathrm{var}}$, in corruptiones TES ${ }^{\mathrm{var}}$, in resurrectione $\mathrm{TES} \mathrm{S}^{\mathrm{var}}$, in interitum $\mathrm{SPM}^{\mathrm{ed}}$, corruptione $\mathrm{SPM}^{\mathrm{var}}$

surgit TES ${ }^{\text {var }}$ ] surget $5158616488^{*}$ AMst $^{\text {var }}$ PEL $^{\text {var }}$ TES $^{\text {var }}$ SPM $^{\text {var }}$, resurgit $\mathrm{TES}^{\mathrm{ed}}$, resurget TES ${ }^{\mathrm{var}}$

in incorruptione ${ }_{5} 861\left(77^{*}\right) \mathrm{AMst}^{\mathrm{D}}$ TE $^{\mathrm{var}}$ ] in incorruptionem 5464757688 $89 \mathrm{AMst}^{\mathrm{A}}$, in incorruptelam AMst ${ }^{\mathrm{ed}}$, in incorruptela AMstrar, sine corruptela $\mathrm{TES}^{\mathrm{ed}}$, in gloria $\mathrm{TES}^{\mathrm{var}} \mathrm{SPM}^{\mathrm{var}}$, sine corruptione $\mathrm{TES}^{\mathrm{var}}$, in perpetuitatem $\mathrm{SPM}^{\mathrm{ed}}$

15:43 Present: 5154586164757677788889 251 AMst PEL TES SPM seminatur in 251 ] seminatur TES ${ }^{\mathrm{var}}$

ignobilitate ] contumelia $64 \mathrm{SPM}^{\text {ed }}$, contumeliam 7576 , contumelia uel ignobilitate 77 , ignominia AMst TES, infirmitate $S_{P M}{ }^{\text {var }}$

surgit ] surget $5158616488^{*} \mathrm{AMst}^{\mathrm{var}} \mathrm{TES}^{\mathrm{var}} \mathrm{SPM}^{\mathrm{var}}$, resurget AMst ${ }^{\mathrm{ed}} \mathrm{TES}^{\mathrm{var}}$, resurgit $\mathrm{TES}^{\mathrm{ed}}$

in gloria ] in gloriam $5^{1} 7^{6} \mathrm{AMst}_{\mathrm{PEL}}^{\mathrm{var}}$, in uirtute $\mathrm{SPM}^{\mathrm{var}}$ seminatur 25

in infirmitate ] lac. 64, in infirmitatem TES ${ }^{\mathrm{var}}$, in contumelia $\mathrm{SPM}^{\mathrm{var}}$ surgit ] surget $515861767788^{*} \mathrm{AMst}^{\mathrm{ADR}} \mathrm{TES}^{\mathrm{var}} \mathrm{SPM}^{\mathrm{var}}$, lac. 64, resurgit TES ${ }^{\text {ed }}$, resurget TES $\mathrm{Sar}^{\mathrm{var}}$

in uirtute ] in uirtutem $51 \mathrm{TES}$ var, lac. 64, in gloria $\mathrm{AMst}^{\mathrm{var}} \mathrm{SPM}^{\mathrm{var}}$

15:44 Present: 51545861757677788889 251 AMst PEL TES SPM seminatur corpus animale (58) 251 ] seminatur corpus carnale TES ${ }^{\text {var }}$ surgit AMst ${ }^{\text {var }}$ TES $^{\text {var }}$ ] surget $51586177^{*}$ AMst $^{\text {ed }}$ TES $^{\text {var }}$, resurgit TES $S^{\text {ed }}$, resurget TES $S^{\text {var }}$

corpus spiritale $5^{1} 5^{8} 77 \mathrm{TES}^{\mathrm{var}} \mathrm{SPM}^{\mathrm{var}}$ ] spiritale $\mathrm{TES}^{\mathrm{ed}} \mathrm{SPM}^{\mathrm{ed}}$, in spiritale TES $^{\text {var }}$ + sicut 77

si est corpus animale $\left.\mathrm{AMst} \mathrm{AD}^{\mathrm{AD}} \mathrm{PEL}{ }^{\mathrm{var}}\right]$ om. $61 \mathrm{AMst}^{\mathrm{ed}} \mathrm{PEL}^{\mathrm{AB}}$ SPM, est igitur corpus animale $\mathrm{AMst}^{\mathrm{R}}$, abs. TES

est et spiritale $\left.5^{1} 5^{8} 77 \mathrm{AMst}^{\mathrm{ADR}} \mathrm{PEL}^{\mathrm{var}}\right]$ om. $61 \mathrm{AMst}^{\mathrm{ed}} \mathrm{PEL}^{\mathrm{AB}}$ SPM, et est spiritale 89 , abs. TES 


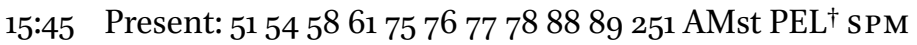
sic $\mathrm{AMst}^{\mathrm{var}} \mathrm{PEL}^{\text {var }}$ ] om. $58 \mathrm{AMst}^{\text {ed }} \mathrm{PEL}^{\mathrm{A}}$, sicut $6177788889 \mathrm{AMst}^{\mathrm{R}} \mathrm{PEL}^{\mathrm{B}}$ et ] om. $5^{1} 5^{861} 77788889 \mathrm{AMst}^{\text {ed }} \mathrm{PEL}{ }^{\mathrm{AB}}$, enim $54 \mathrm{AMst}^{\mathrm{var}} \mathrm{PEL}^{\mathrm{var}}$, etiam 75 $76 \mathrm{SPM}$

scriptum est $89{ }_{25}$ AMst $^{\mathrm{var}}$ ] om. AMst $^{\text {ed }} \mathrm{PEL}^{\mathrm{A}} ;++$ enim 58

factus est primus homo adam in

animam uiuentem ] om. $54^{*}$, animam uiuificantem $61^{*}$, anima uiuente $S_{P M}{ }^{\text {var }}$

nouissimus ] om. $54^{*}$, secundus $\mathrm{SPM}^{\mathrm{var}}$; + autem AMst PEL var

adam in ] om. $54^{*}$

spiritum uiuificantem $\mathrm{SPM}^{\mathrm{var}}$ ] spiritu uiuificantem $5461^{*} 76^{*} 778889$

$\mathrm{AMst}^{\mathrm{var}} \mathrm{PEL}{ }^{\mathrm{var}}$, animam uiuificantem 58 , spiritu uiuificante $757^{6} \mathrm{SPM}^{\text {ed }}$

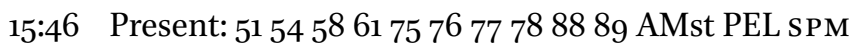

sed non ] om. PEL ${ }^{\mathrm{A}}$, seminatur non $\mathrm{SPM}^{\mathrm{var}}$

prius $\mathrm{SPM}^{\mathrm{var}}$ ] primum AMst $\mathrm{SPM}^{\mathrm{ed}}$, om. $\mathrm{PEL}^{\mathrm{A}} \mathrm{SPM}^{\mathrm{var}}$

quod spiritale est $\left.515^{8}(75)\right]$ spiritale 77, om. $\mathrm{AMst}^{\mathrm{AD}}$, om. $\mathrm{PEL}^{\mathrm{A}}$

sed quod animale ] sed animale $77, \mathrm{om}$. $\mathrm{AMst}^{\mathrm{AD}} \mathrm{PEL}^{\mathrm{A}}$, sed quod amabile $S^{\text {var }}$

est PEL var SPM $^{\text {var }}$ ] om. 75767778 AMst PEL $^{\mathrm{AB}}$ SPM $^{\text {ed }}$

deinde quod spiritale $5158 \mathrm{AMst}^{\mathrm{AD}}$ ] deinde spiritale $77 \mathrm{SPM}^{\mathrm{ed}}$, om. AMsted $\mathrm{PEL}^{\mathrm{A}}$, deinde quod finis SPM ${ }^{\mathrm{var}}$; + est $5154586189 \mathrm{AMst}^{\mathrm{AD}} \mathrm{PEL}^{\mathrm{var}}$

15:47 Present: 51545861757677788889251 AMst PEL tes

primus homo ] fratres primus homo 251, hic primus homo PEL var

de terra terrenus ] de terrae limo $\mathrm{TES}^{\mathrm{ed}}$, de terra e limo $\mathrm{TES}^{\mathrm{var}}$, de terra limo TES ${ }^{\mathrm{var}}$

secundus homo ] secundus PEL var

de $\mathrm{AMst}^{\mathrm{AD}}$ ] e AMsted $\mathrm{TES}^{\text {var }}$

caelo 51545876777888 ] om. $89^{*}$

caelestis 515458777888 ] om. $7576 \mathrm{TES}$

15:48 Present: 51545861757677788889251 AMst PEL tes

qualis ] quales TES ${ }^{\text {var }}$; + est AMst

terrenus $\mathrm{AMst}^{\mathrm{AD}}$ ] terrenus uel terrestris 77 , ille terrestris $\mathrm{AMst}^{\text {ed }}$, ille e limo $\mathrm{TES}^{\text {ed }}$, ille de limo $\mathrm{TES}^{\mathrm{var}}$, ille qui de limo est TES ${ }^{\mathrm{var}}$

$\dagger{ }_{1}$ Cor. 15:45. The first half of this verse appears in different places in different manuscripts of PEL. 
tales ] talis $6175251 \mathrm{TES}^{\mathrm{var}}$

et terreni $\mathrm{AMst}^{\mathrm{AD}}$ ] terreni uel terrestres 77, et terrestres $\mathrm{AMst}^{\mathrm{ed}}$, et qui de limo $\mathrm{TES}^{\mathrm{ed}}$, qui de terrae limo TES $\mathrm{S}^{\mathrm{var}}$

et qualis caelestis $51545^{8} 767788 \mathrm{AMst}^{\mathrm{AD}}$ ] et qualis ille caelestis $\mathrm{AMst}^{\mathrm{ed}}$, et qualis e caelis TES $\mathrm{Tar}^{\mathrm{v}}$

tales et ] talis et 7589 TES $^{\text {var }}$

caelestes 51545861767778 ] caelestis 75 TES $^{\text {var }}$; + de caelo TES ${ }^{\text {var }}$

15:49 Present: 51545861757677788889251 AMst PEL TES

igitur $\mathrm{AMst}^{\mathrm{AD}}$ ] om. 75767789 AMst $^{\mathrm{ed}}$ TES

sicut ] quomodo TES

portauimus imaginem $\left.51545^{8}\langle 76\rangle\right]$ portabimus imaginem TES ${ }^{\text {var }}$

terreni ] illius terreni $61 \mathrm{AMst}^{\mathrm{var}} \mathrm{PEL}^{\mathrm{B}}$, terrestris 7576 , eius qui de limo est TES ${ }^{\text {ed }}$, eius qui de caelo est TES $S^{\text {var }}$, illius qui e terra est $\mathrm{TES}^{\mathrm{var}}$

portemus et ] + in $\mathrm{PEL}^{\mathrm{B}}$

imaginem $51545^{8}$ ] + eius $61 \mathrm{AMst}^{\text {ed }}$ TES, + huius $\mathrm{PEL}^{\mathrm{B}}$

caelestis $515458767788 \mathrm{AMst}^{\mathrm{AD}}$ ] qui de caelo est $\mathrm{AMst}^{\mathrm{ed}} \mathrm{TES}^{\text {ed }}$, qui de limo est TES $S^{\text {var }}$

15:50 Present: $5^{1} 545^{8} 61757677788889251$ AMst PEL

hoc autem $\mathrm{AMst}^{\mathrm{AD}}$ ] hoc enim $75767789 \mathrm{AMst}^{\text {ed }}$

dico fratres ] fratres dico 58 , dico uobis 61

quoniam ] quia $7576777889 \mathrm{AMst} \mathrm{PEL}^{\mathrm{A}}$

caro et sanguis regnum dei $\langle 77\rangle$

possidere non possunt 251 ] non possidebunt $6178 \mathrm{AMst}^{\mathrm{ed}} \mathrm{PEL}^{\mathrm{B}}$, non here-

ditabunt uel possidebunt 77 , possidere non possidebunt $\mathrm{AMst}^{\mathrm{AD}}$, non possidebit $P E L^{\text {var }}$

neque corruptio 5861 ] om. $\mathrm{PEL}^{\mathrm{A}}$

incorruptelam ] incorruptionem $61, \mathrm{om}$. $\mathrm{PEL}^{\mathrm{A}}$

possidebit $\left.6{ }^{c}{ }^{\mathrm{PEL}}{ }^{\mathrm{ed}}\right]$ uidebit $61^{*}$, om. $\mathrm{PEL}^{\mathrm{A}}$, possidebunt $\mathrm{PEL}^{\mathrm{B}}$

15:51 Present: $3^{2} 5^{1} 545^{8} 617576777^{8} 888925^{\dagger}$ AMst PEL SPM

\pm fratres $25^{2}$

ecce mysterium uobis dico $\left.545861882_{251^{1}} \mathrm{PEL}^{\mathrm{var}}\right]$ abs. 32, ecce mysterium dico uobis $89 \mathrm{AMst}^{\mathrm{AD}}$, ecce mysterium dico $\mathrm{AMst}^{\text {ed }} \mathrm{PEL}{ }^{\mathrm{AB}}$, ecce dico uobis mysterium SPM

omnes quidem ] fratres omnes quidem 32, omnes 757689

$\dagger{ }_{1}$ Cor. 15:51. A second lection in VL 251 starts with this verse, covering 15:51-58. 
resurgemus $54 \mathrm{SPM}^{\mathrm{var}}$ ] resurgimus $32757689251 \mathrm{PEL}^{\mathrm{var}} \mathrm{SPM}^{\mathrm{ed}}$, dormiemus 77

sed non omnes ] non omnes autem 7576 77, non omnes PEL ${ }^{\mathrm{B}}$

inmutabimur 51 (54) $58617576777825^{1}$

15:52 Present: $3^{2} 5^{1} 545^{8} 61757677788889251$ AMst PEL SPM

in momento $\left.\left(88^{*}\right)\right]+$ et $58 \mathrm{SPM}^{\mathrm{var}}$

in ictu $\left.3225^{1}\right]+$ uel nutu 77

oculi in nouissima tuba 5478

canet enim PEL ${ }^{\text {var }}$ ] canit enim 32, canet enim tuba $5^{1} 545^{8} 78$ AMst $^{\text {ADR }}$

PEL $^{\text {var }}$ SPM, bucinat enim 7576 , tubicinatur uel tuba enim canitur 77 , tubi-

cinat autem 89, om. AMst ${ }^{\text {ed }} \mathrm{PEL}^{\mathrm{B}}$, canent enim PEL ${ }^{\mathrm{A}}$

et ] om. PEL ${ }^{\mathrm{B}}$

mortui resurgent 54 ] resurgent mortui 61 , mortui resurgunt $75 \quad 76{ }^{25} \mathbf{1}^{1}$

$\mathrm{AMst}^{\mathrm{A} * \mathrm{D} *} \mathrm{PEL}^{\mathrm{var}} \mathrm{SPM}^{\mathrm{var}}$, mortui surgent 78

incorrupti 61 ] om. PELA

et nos inmutabimur $515861(77) 8925^{1}$ ] et nos mutabimur 7576

15:53 Present: $3^{2} 5^{1} 545^{8} 61757677788889$ 251 AMst PEL TES SPM

oportet enim ] oportet ergo TES ${ }^{\mathrm{var}}$, oportet TES ${ }^{\mathrm{var}}$

corruptibile hoc 61 ] hoc corruptibile 51, incorruptibile hoc PEL ${ }^{\mathrm{A}}$, corruptiuum istud TES ${ }^{\text {ed }}$, corruptiuum TES ${ }^{\text {var }}$, corruptum hoc TE $S^{\text {var }}$

induere $(32)$

incorruptelam $3^{2} \mathrm{PEL}^{\mathrm{A}}$ ] incorruptionem $5^{1} 617576777888$ 89 AMst PEL var

$\mathrm{TES}^{\mathrm{ed}} \mathrm{SPM}^{\mathrm{ed}}$, corruptionem TES ${ }^{\mathrm{var}}$, inmortalitatem TES $\mathrm{Sar}^{\mathrm{var}}$, incorruptione $\mathrm{SPM}^{\mathrm{var}}$

et mortale hoc ] et mortale 77 , et mortale istud $\mathrm{TES}^{\mathrm{var}}$

induere inmortalitatem 54767778 AMst ] induere inmortalitate $\mathrm{SPM}^{\mathrm{var}}$

15:54 Present: $3^{2} 5^{1} 545^{8} 61757677788889$ 251 AMst PEL TES

cum autem TES ${ }^{\text {var }}$ ] om. 7778 TES $^{\text {ed }}$, cum ergo AMst ${ }^{\text {var }}$

\pm corruptio hoc induerit incorruptionem et $54^{*}$, corruptibile hoc induerit incorruptionem et $54^{\mathrm{C}} 58$, corruptibile hoc induerit incorruptalitatem et 7576 , corruptum hoc induerit in corruptionem et PEL ${ }^{\text {var }}$, corruptiuum hoc induerit incorruptionem et $\mathrm{TE} \mathrm{S}^{\mathrm{var}}$

mortale hoc TES ${ }^{\text {var }}$ ] om. 7778 TES $^{\text {ed }}$, hoc mortale $251^{2 *}$

induerit TES ${ }^{\text {var }}$ ] induere 7576 , om. 7778 TES $^{\text {ed }}$, induerit in 89 , indueret 251

inmortalitatem $3276 \mathrm{TES}^{\mathrm{var}}$ ] om. $7778 \mathrm{TES}^{\mathrm{ed}}$, incorruptionem TES $\mathrm{S}^{\mathrm{var}}$

tunc fiet ] tunc fiat TE $S^{\text {var }}$ 
sermo qui scriptus est $251 \mathrm{AMst}^{\mathrm{AD}} \mathrm{TES}^{\text {var }}$ ] uerbum quod scriptum est $\mathrm{AMst}^{\text {ed }} \mathrm{TES}^{\mathrm{ed}}$, uerbum quod dictum TES ${ }^{\mathrm{var}}$; + in osee propheta 61

absorta est mors $51586177\left(88^{*}\right)$ TES $^{\text {var }}$ ] absumpta est mors TES ${ }^{\text {ed }}$, ubi et mors TES ${ }^{\text {var }}$

in uictoria $89^{*} 25^{1}$ ] uictoria 58 , in uictoriam $\mathrm{AMst}^{\mathrm{ed}}$, om. $\mathrm{AMst}^{\mathrm{A} * \mathrm{D} *}$, in contentionem TES $S^{\text {ed }}$, in contentione TES ${ }^{\text {var }}$, sine contentione TES ${ }^{\text {var }}$

15:55 Present: $3^{2} 5^{1} 545^{8} 61757677788889$ 251 AMst PEL tes

ubi est mors ] ubi tuus mors 77, om. AMst ${ }^{\mathrm{A} * \mathrm{D} *}$, ubi est mors ubi est TES $\mathrm{S}^{\mathrm{var}}$ uictoria tua $25^{1} \mathrm{AMst}^{\mathrm{AD}} \mathrm{PEL}^{\text {var }}$ ] contentio tua 61 , stimulus tuus $7576 \mathrm{PEL}^{\mathrm{AB}}$, aculeus 77 , aculeus tuus 78 AMst $^{\text {ed }}$ TES

ubi est mors ] ubi tua o mors 77 , ubi mors $88^{*}$, om. PEL $^{\mathrm{A}}$, ubi est TES ${ }^{\mathrm{var}}$ stimulus tuus PEL ${ }^{\text {var }}$ ] aculeus tuus $54618925^{2}$ AMst $^{\mathrm{AD}}$, uictoria tua 7576 $78 \mathrm{PEL}^{\mathrm{B}}$, uictoria 77 , contentio tua AMst ${ }^{\mathrm{ed}}$ TES, om. PEL ${ }^{\mathrm{A}}$

15:56 Present: $3^{2} 5^{1} 545^{8} 61757677788889$ 251 AMst PEL

stimulus autem mortis ] aculeus autem mortis (54) $6175767778892 \mathbf{2 1}^{2}$

AMst

peccatum est $A M s t^{\text {var }}$ ] peccatum $75767789 \mathrm{AMst}^{\text {ed }}$, est peccatum $\mathrm{PEL}^{\mathrm{B}}$ uirtus uero $\left.61^{\text {alt }}\right]$ uirtus autem 61757677 AMst

peccati lex (88) ] peccati $\mathrm{PEL}^{\mathrm{B}}$

15:57 Present: $3^{2} 5^{1} 545^{8} 61757677788889$ 251 AMst PEL

deo autem gratias 58251 ] sed deo gratias 61, deo gratias 77 qui dedit $\mathrm{AMst}^{\mathrm{AD}}$ ] danti uel qui dedit 77 , qui tribuit $\mathrm{AMst}^{\mathrm{ed}}$ nobis uictoriam $25^{1}$ ] uobis uictoriam 767778

per dominum nostrum iesum christum (76) ] per iesum christum dominum nostrum $5^{1}$

15:58 Present: $3^{2} 5^{1} 545^{8} 617576777^{8} 8889$ 251 AMst PEL SPE

itaque fratres mei 32 ] itaque fratres 5189

dilecti $32 \mathrm{AMst}^{\mathrm{AD}} \mathrm{PEL}^{\text {var }}$ ] dilectissimi $51 \mathrm{AMst}^{\mathrm{ed}}$, om. $89 \mathrm{PEL}^{\mathrm{AB}}$

stabiles estote ] estote stabiles $58 \mathrm{SPE}^{\mathrm{var}}$

et $\mathrm{AMst}^{\mathrm{AD}}$ ] om. $75767789 \mathrm{AMst}^{\mathrm{ed}} \mathrm{PEL}^{\mathrm{B}}$

inmobiles abundantes in 32515458617576778889

opere domini ] opera dei 89, opere $\mathrm{AMst}^{\mathrm{var}}$, opere dei PEL var semper scientes

quod ] quia 75767789 AMst $^{\text {var }}$, om. PEL var

labor uester non est ] non est labor uester 77, non est PEL var inanis in domino ] inanis domino AMst ${ }^{\mathrm{var}}$ 


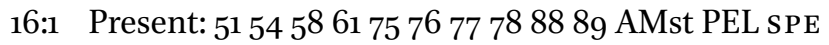

de collectis autem quae 515458617688 ] de collectione uel collectis uel autem 77 , nam de collectis quae $\mathrm{PEL}^{\mathrm{B}}$, quae $\mathrm{PEL}^{\text {var }}$

fiunt ] om. 77, fient PELA

in sanctos $\mathrm{AMst}^{\mathrm{A}}$ ] in sanctis $5475767888^{*} 89 \mathrm{AMst}^{\mathrm{ed}} \mathrm{PEL}^{\mathrm{B}} \mathrm{SPE}^{\mathrm{var}}$

sicut ordinaui 54 ] sicut ordinauit $75 \mathrm{PEL}^{\text {var }}$

ecclesiis $5^{1} 61$ ] ecclesiae $\mathrm{AMst}^{\mathrm{var}} \mathrm{PEL}^{\mathrm{B}}$, in ecclesiis $\mathrm{SPE}^{\mathrm{var}}$

galatiae 51545888 ] galitiae 61

ita et uos facite

16:2 Present: $5^{1} 545^{8} 61757677788889$ AMst $^{\dagger}$ PEL SPE

per ] per uel secundum 77

unam ] unamquamque $54^{\mathrm{C}} 5^{8}$

sabbati unusquisque uestrum apud se $515^{8} 7589$

ponat ] reponat $51545^{8} \mathrm{PEL}^{\mathrm{var}}$, om. $\mathrm{PEL}^{\mathrm{B}}$

recondens ] thesaurizans 75767789 AMst $^{e d}$, thesaurizat $\mathrm{AMst}^{\mathrm{A} * \mathrm{D} \text { * }}$

quod ei ] quodcumque AMst

beneplacuerit ] placuerit $757689 \mathrm{PEL}^{\mathrm{A}}$, uoluerit AMst

ut ] et PEL ${ }^{\text {var }}, o m$. PEL ${ }^{\mathrm{A}}$

non cum uenero ] cum uenero non 58 , om. PEL ${ }^{\mathrm{A}}$

tunc collectae fiant 515458618889 ] tunc collecta fiant $7576 \mathrm{PEL}^{\mathrm{var}} \mathrm{SPE}^{\mathrm{var}}$, tunc collectae uel collecta fiant $77, \mathrm{om}$. $\mathrm{PEL}^{\mathrm{A}}$

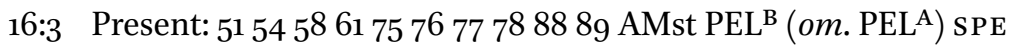
cum autem

praesens fuero $51545^{8} 61 \mathrm{PEL}^{\text {var }}$ ] aduenero $757689 \mathrm{AMst}^{\text {ed, }}$, aduenero uel praesens fuero 77 , uenero $\mathrm{AMst}^{\mathrm{AD}}$, fuero praesens $\mathrm{PEL}^{\mathrm{B}}$

quos $\mathrm{AMst}{ }^{\mathrm{AD}} \mathrm{PEL}^{\text {var }}$ ] quoscumque $6175767789 \mathrm{AMst}^{\text {ed }} \mathrm{PEL}^{\mathrm{B}}$

probaueritis per epistulas $617576777888 \mathrm{PEL}^{\text {var }}$ ] probaueritis per epistulam $89 P E L^{B}$

hos mittam perferre ] hos mittam perficere PEL var

gratiam uestram 58

in hierusalem $\left.545^{8} 7677 \mathrm{PEL}^{\text {var }}\right]$ hierusalem $\mathrm{PEL}^{\mathrm{B}}$

16:4 Present: $5^{1} 545^{8} 61757677788889$ AMst PEL $^{\mathrm{B}}$ (om. PEL ${ }^{\mathrm{A}}$ ) SPE

quod si dignum fuerit ut $\mathrm{AMst} \mathrm{t}^{\mathrm{AD}}$ ] si autem uel quod si fuerit dignum ut 77 , si uero dignum fuerit ut AMsted, quod signum fuerit PEL var

$\dagger \quad 1$ Cor. 16:2. Most of this verse is missing from four manuscripts of AMst, which also omit the latter part of 16:7. 
et ego PEL ${ }^{\mathrm{var}} S \mathrm{PE}^{\mathrm{var}}$ ] ego $5^{1} 545^{8} 77 \mathrm{PEL}^{\mathrm{B}} \mathrm{SPE}^{\mathrm{ed}}$, ego quoque $\mathrm{AMst}{ }^{\mathrm{AD}}$ eam mecum ibunt $\left(77^{*}\right) \mathrm{AMst}^{\mathrm{AD}}$ ] eam mecum ibant $89^{*}$, eam ibunt mecum AMst $^{\text {ed }}$

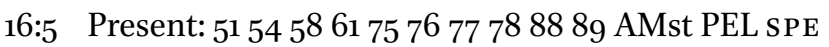

ueniam ] uenio 7576

autem ad uos cum ] ad uos cum AMst ${ }^{\text {var }}$, ad uos autem cum $\mathrm{PEL}^{\mathrm{B}}$

macedoniam 61758889 SPE ] macedonia PEL ${ }^{\text {var }}$ + enim AMst ${ }^{\mathrm{AD}}$

pertransiero $\left.\left(58^{*}\right)\right]$ transiero $7576 \mathrm{AMst}^{\text {var }}$

nam macedoniam pertransibo $88 S P E$ ] nam pertransibo macedoniam 61 , macedoniam enim pertranseo $7589 \mathrm{AMst}^{\mathrm{ed}}$, macedonia enim pertranseo 76, nam macedoniam enim pertransibo 77, om. $\mathrm{AMst}^{\mathrm{AD}} \mathrm{PEL}^{\mathrm{var}}$

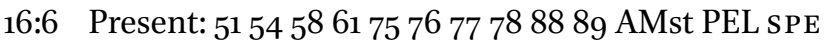

apud uos 5154587589

autem $S P E^{\text {var }}$ ] om. AMst ${ }^{\text {var }}$ PEL ${ }^{\text {var }}$, etiam SPE ${ }^{\text {ed }}$

forsitan 7589 ] forte AMst ${ }^{\text {var }}$, necesse est PEL ${ }^{\mathrm{A}}$

manebo AMst ${ }^{\text {var }}$ SPE ${ }^{\text {var }}$ ] remanebo $61757^{\mathrm{C}} 7789 \mathrm{AMst}^{\mathrm{ed}} \mathrm{PEL}^{\mathrm{var}}$ SPE ${ }^{\text {ed }}$, hiemabo remanebo $76^{*}$, ut maneam $\mathrm{PEL}^{\mathrm{A}}$

uel etiam AMst ${ }^{\text {var }}$ ] et 6177 , aut etiam AMsted, uel PELA

hiemabo 51 ] hibernabo uel hiemabo 77 , hiemem PELA

ut AMst ${ }^{\text {var }}$ ] nisi 6177 AMst $^{\text {ed }}$ PEL $^{\text {var }}$, nisi si 7576 89, om. PEL ${ }^{\mathrm{A}}$

uos me $\mathrm{AMst}^{\mathrm{var}}$ ] me uos 61, uos forte AMsted, me AMst ${ }^{\mathrm{AD}} \mathrm{PEL}^{\mathrm{var}}$, om. PEL ${ }^{\mathrm{A}}$ deducatis $\mathrm{AMst}^{\mathrm{AD}}$ ] ducatis $5^{1} \mathrm{PEL}^{\mathrm{B}}$, duxeritis 7576 , deduxeritis 7789 $\mathrm{AMst}^{\mathrm{ed}} \mathrm{PEL}{ }^{\mathrm{var}}$, om. PELA

quocumque iero 77 ] ubicumque iero 7576 89, om. $\mathrm{PEL}^{\mathrm{A}}$

16:7 Present: $5^{1} 545^{8} 61757677788889 \mathrm{AMst}^{\dagger} \mathrm{PEL}^{\mathrm{B}}$ (om. $\mathrm{PEL}^{\mathrm{A}}$ ) SPE

nolo enim uos

modo PEL var ] nunc $757689 \mathrm{PEL}^{\mathrm{B}}$, nunc uel modo 77

in transitu uidere $\mathrm{SPE}^{\mathrm{var}}$ ] uidere in transitu 61, in transitum uidere $89 \mathrm{SPE}^{\mathrm{ed}}$ spero enim me ] spero enim 757677 , spero autem me SPE ${ }^{\text {var }}$

aliquantum temporis ] aliquantulum temporis $5158 \mathrm{AMst}$, aliquid temporis

61 , aliquod temporis $75\langle 76\rangle 89$, tempus aliquod 77

manere apud uos PEL var $]$ mansurum apud uos $61 \mathrm{PEL}^{\mathrm{B}}$

si dominus permiserit 51586175$]$ si dominus uoluerit 54

$\dagger \quad{ }_{1}$ Cor. 16:7. The same four manuscripts of AMst which omit most of $16: 2$ also omit the latter half of this verse. 
16:8 Present: 51545861757677788889 AMst PEL SPE

permanebo ] manebo $61 \mathrm{PEL}^{\mathrm{B}}$

autem ] enim SPE $\mathrm{var}^{\mathrm{v}}$

ephesi $61 A M s t^{e d}$ ] ephesi uel in epheso 77 , ephesios $A M s t^{A * D *}$

usque ad pentecosten 586177 ] ad pentecosten $A M s t^{A * D *}$

16:9 Present: 51545861757677788889 AMst PEL SPE

ostium enim mihi 5177

apertum est magnum $\mathrm{AMst}^{\mathrm{var}}$ ] magnum apertum est $\mathrm{AMst}^{\mathrm{ed}}$, apertum est PEL var

et ] om. PEL var

euidens ] uidens 75, operosum $\mathrm{AMst}^{\mathrm{AD}}$, efficax $\mathrm{AMst}^{\mathrm{ed}}$

et $S P E^{\text {var }}$ ] sed $61 \mathrm{PEL}^{\mathrm{B}}$, om. $S P E^{\mathrm{ed}}$

aduersarii multi

16:10 Present: 51545861757677788889 AMst PEL sPE

si autem $\mathrm{AMst}^{\mathrm{AD}}$ ] quod si $7576 \mathrm{AMst}^{\mathrm{ed}}$, cum autem 89

uenerit timotheus 5154586189

uidete ] uidere $\mathrm{PEL}^{\mathrm{B}}$

ut sine ] ne PEL ${ }^{\text {var }}$, et sine PEL ${ }^{\text {var }}$, sine $S P E^{\text {var }}$

timore ] in timore PEL ${ }^{\text {var }}$, timorem PEL var

sit apud uos 515875 ] apud uos sit PEL var

opus enim domini ] nam opus domini $61 \mathrm{PEL}^{\mathrm{B}}$, abs. SPE

operatur sicut et ego ] operatur sicut et ego operor 61, operatur sicut ego 88

$\mathrm{PEL}^{\mathrm{var}}$, sicut et ego operatur AMst ${ }^{\mathrm{AD}}$, abs. SPE

16:11 Present: 515458617576777888 89 AMst PEL

ne quis ergo illum PEL var ] ne quis illum $6175767789 \mathrm{AMst}^{\text {ed }} \mathrm{PEL}^{\mathrm{AB}}$, ne quis illum ergo $88^{*}$, ergo ne quis illum $\mathrm{AMst}^{\mathrm{AD}}$, ne quis enim illum PELvar

spernat deducite ] spernat praemittite $75>677$

autem illum ] illum $A M s t^{R}$; + ad me $61^{*}$

in pace ] cum pace $89 \mathrm{PEL}^{\mathrm{A}}$

ut ueniat ad me ] ueniat ad me $54^{*}$

expecto enim 7677 ] expecto AMst ${ }^{\text {var }}$ PEL $^{\text {var }}$, expecto autem PEL var

illum ] om. $5^{1 ;}+$ in epheso $5^{8}$

cum fratribus ] om. PELA

16:12 Present: $5^{1} 545^{8} 61647576777888$ 89 AMst PEL ${ }^{\mathrm{B}}$ (om. PEL ${ }^{\mathrm{A}}$ )

de apollo autem 61 ] lac. 64 
fratre PEL ${ }^{\text {var(ed) }}$ ] fratre nostro 61, lac. 64, fratrem 89, fratres $P E L^{B}$

\pm notum facio quoniam 51 , significo uobis quia 5478 AMst PEL ${ }^{\text {var, notum }}$ uobis facio quoniam 5888 , notum uobis faciam quoniam 61 , notum uobis facio quia $7576 \mathrm{PEL}^{\mathrm{B}}$, notesco uobis quia 77

multum ] lac. 64, multum uel multa 77, om. PEL var

rogaui eum $\mathrm{PEL}^{\mathrm{var}}$ ] illum rogaui $61757689 \mathrm{AMst}^{\mathrm{ed}} \mathrm{PEL}^{\mathrm{B}}$, rogaui 64 , rogaui illum 77 AMstvar

ut ueniret ad uos $P E L^{\text {var(ed) }}$ ] ut uenirent ad uos $P E L^{B}$

cum fratribus et ] cum fratribus $89^{*}$

utique ] omnino 6477 , unicuique PEL ${ }^{\text {var }}$

non fuit ] + uel erat 77

uoluntas ] uolumptas 54, uoluptas 75, om. PEL $;$; + eius 515458 AMstrar $^{\text {var }}$ $\mathrm{PEL}^{\mathrm{B}}$

ut nunc ] ut 61, nunc 77, et nunc PEL var

ueniret ] ueniat $\mathrm{AMst}^{\mathrm{AD} *}$

ueniet autem cum ] ueniat autem cum PEL var

ei ] om. $6175767789 \mathrm{AMst}^{\mathrm{AD}} \mathrm{PEL}^{\mathrm{B}}$, et 64

uacuum ] opportunum uel uacuum 58 , opportunum $616475767789 A M s t^{A D}$ $P E L^{B}$

fuerit ] fuit PEL var

16:13 Present: 5154586164757677788889411 AMst PEL SPM SPE

\pm fratres 411

uigilate 61 ] + et $586488 \mathrm{SPE}^{\mathrm{var}}$, + et orate $411^{*}$, + et orate et $411^{\mathrm{C}}$

state in fide (77) ] in fide $61 \mathrm{PEL}^{\mathrm{B}} \mathrm{SPM}^{\mathrm{var}}$

uiriliter agite et confortamini ] uiriliter agite confortamini $\left(54^{*}\right)^{\mathrm{C}} 647789$ $\mathrm{AMst}^{\text {ed }} \mathrm{PEL}^{\mathrm{var}}$, confortamini uiriliter agite $7576 \mathrm{SPM}$, uiriliter agite corroboramini $\mathrm{AMst}^{\mathrm{AD}}$; + in domino 58

16:14 Present: 5154586164757677788889411 AMst PEL SPM SPE

omnia ] + enim PEL ${ }^{\mathrm{A}}$

uestra in ] uestra cum $6164 \mathrm{PEL}^{\text {var(ed) }} \mathrm{SPE}^{\mathrm{var}}$

caritate fiant 411 ] caritatem fiant 64

16:15 Present: 51545861647576777888 89 AMst PEL ${ }^{\mathrm{B}}$ (om. PEL ${ }^{\mathrm{A}}$ )

obsecro autem uos fratres

nostis $\mathrm{AMst}^{\mathrm{AD}}$ ] scitis $\mathrm{AMst}^{\mathrm{ed}}$

domum stephanae 5154586164777888

et fortunati ] et fortunati et achaici $54617778 \mathrm{PEL}^{\mathrm{B}}$, et achaici et fortunati 58, om. $64 \mathrm{AMst}^{\mathrm{var}}$ 
quoniam ] quia AMst ${ }^{\text {var }} ;+$ qui $5^{8}$

sunt primitiae $51545888 \mathrm{AMst}^{\mathrm{var}} \mathrm{PEL}^{\mathrm{var}}$ ] est initium 64, sunt initium 7576 $89 \mathrm{AMst}^{\mathrm{ed}}$, primitiae sunt $\mathrm{PEL}^{\mathrm{B}}$

achaiae et $\left.5154586188 \mathrm{AMst}^{\mathrm{AD}}\right]$ achaiae et se $\mathrm{AMst}^{\mathrm{ed}}$

in ministerium ] in ministerio PEL var

sanctorum ] sanctis $757^{6} 77 \mathrm{AMst}^{\mathrm{var}}$, om. 89, sanctis fratribus PEL var

ordinauerunt se ipsos $\mathrm{AMst}^{\mathrm{AD}} \mathrm{PEL}^{\text {var }}$ ] se ordinauerunt $61 \mathrm{PEL}^{\mathrm{B}}$, constituerunt uel ordinauerunt se ipsos 77 , ordinauerunt $\mathrm{AMst}^{\mathrm{ed}}$, se ipsos ordinauerunt PEL ${ }^{\text {var }}$ + sanctis fratribus 89

16:16 Present: 5154586164757677788889 AMst PEL $^{\mathrm{B}}$ (om. PEL $\left.{ }^{\mathrm{A}}\right)$

ut et uos

subditi sitis $78 \mathrm{PEL}^{\text {var }}$ ] subiecti sitis $647576 \mathrm{PEL}^{\mathrm{B}}$; + uel subdatis 77

eiusmodi et omni AMst ${ }^{\mathrm{AD}} \mathrm{PEL}^{\text {var }}$ ] talibus et omni 616475767789 AMst $^{\text {ed }}$ PEL $^{\mathrm{B}}$

cooperanti et laboranti 54 ] qui adiuuat et laborat 757689 , operanti et laboranti AMst ${ }^{\text {var }}$, operanti et conlaboranti PEL ${ }^{\text {var }}$; + in uobis $545^{861777889}$ PEL $^{\mathrm{B}}$

16:17 Present: 51545861647576777888 89 AMst PEL

gaudeo autem in ] gaudeo in (88)

praesentia $515488 \mathrm{AMst}^{\mathrm{var}}$ ] perseuerantia 58 , aduentum $6189^{*}$, aduentu 75 $7689^{\mathrm{C}} \mathrm{AMst}^{\mathrm{ed}}$, aduentu uel praesentia 77

stephanae et $5154586164767788 P E L^{A}$ ] stephani et PEL ${ }^{\mathrm{var}}$

fortunati et 7788 ] fortunati 51 , furtinati et 61

achaici $77^{c} 78$ ] achaiae $64 \mathrm{PEL}^{\text {var }}$, achaikou $77^{*}$

quoniam id quod $\mathrm{AMst}^{\mathrm{A}}$ ] quia quod $64757677 \mathrm{AMst}^{\mathrm{ed}}$, quoniam quod

89

uobis $\langle 51\rangle]$ a uobis 647576

deerat ] desse fuit 77

ipsi ] illi $647576 \mathrm{AMst}^{\mathrm{AD}}$, illi uel ipsi 77

suppleuerunt 6489 ] adimpleuerunt $61 \mathrm{PEL}^{\mathrm{B}}$

16:18 Present: 5154586164757677788889 AMst PEL

refecerunt 61 ] perfecerunt 89

enim ] autem $P E L^{B}$

et $\mathrm{AMst}^{\mathrm{AD}}$ ] om. $61 \mathrm{AMst}^{\mathrm{ed}}$

meum spiritum ] meum ẹt spiritum $54^{*}$, spiritum meum 58 AMst

et uestrum cognoscite $\mathrm{AMst}^{\mathrm{AD}}$ ] et uestrum agnoscite $\mathrm{AMst}^{\mathrm{ed}}$

ergo ] + eos $6164 \mathrm{AMst}^{\mathrm{AD}}$ 
qui eiusmodi sunt $\mathrm{AMst}^{\mathrm{A}}$ ] qui huiusmodi sunt $6164 \mathrm{AMst}^{\mathrm{D}} \mathrm{PEL}^{\text {var }}$, huiusmodi AMsted, eiusmodi PEL ${ }^{B}$

16:19 Present: $51545^{8} 6164757677788889$ AMst PEL

salutant uos ] om. PELA; + omnes 58

ecclesiae 515458617678 ] om. PELA

asiae 5154586188 ] sanctae AMst ${ }^{\mathrm{var}}$, achaiae PEL ${ }^{\mathrm{var}}$, om. $\mathrm{PEL}^{\mathrm{A}}$

salutant uos in domino

multum aquila ] aquila multum $64757689 \mathrm{PEL}^{\mathrm{B}}$, multa uel multum aquila 77, aquila AMst ${ }^{\text {var }}$

et prisca 88 ] et priscilla 546175767778 88 alt 89 AMst PEL

cum domestica sua ecclesia 5161 ] cum domestica eorum ecclesia 64, cum

domestica ecclesia sua $\mathrm{AMst}^{\mathrm{var}}$, cum ea quae in domo eorum est ecclesia $\mathrm{PEL}^{\mathrm{B}}$

\pm apud quos et hospitor 51547778 , apud quos et ego hospitor 58 , apud quos etiam hospitor $617576 \mathrm{PEL}^{\mathrm{B}}$, apud aliter quos etiam hospitor 89

16:2O Present: $5^{1} 545^{8} 61647576777888$ AMst PEL $^{\mathrm{B}}$ (om. PEL ${ }^{\mathrm{A}}$ )

salutant uos ] salutent uos 88

fratres omnes ] omnes fratres $5^{8} 75$

salutate inuicem in osculo sancto ] salutate inuicem in amorem sancti 7576

16:21 Present: $5^{1} 545^{8} 6164757677788889$ AMst PEL $^{\mathrm{B}}$ (om. PEL ${ }^{\mathrm{A}}$ )

salutatio mea 58

manu pauli ] in manu pauli $58 \mathrm{PEL}^{\mathrm{var}}$

16:22 Present: $51545^{8} 61647576777888$ 89 AMst PEL

si quis non amat ] si quid non amat $\mathrm{AMst}^{\mathrm{var}}$

dominum iesum christum $\mathrm{AMst}^{\mathrm{AD}}$ ] dominum nostrum iesum christum 51 $5^{8} 6189 \mathrm{PEL}^{\mathrm{B}}$, dominum 64, iesum dominum $\mathrm{AMst}^{\mathrm{ed}}$, dominum iesum AMstvar

sit anathema ] siat anathema 75 , sit perditio 77 , anathema 89 , anathema sit PEL ${ }^{A}$

maranatha (54) 64 ] in aduentu domini 77

16:23 Present: $5^{1} 545^{8} 6164757677788889$ AMst PEL

gratia domini 58

iesu ] nostri iesu christi 515861648889 AMst $^{\text {ed }}$, nostri $54 \mathrm{AMst}^{\text {var }} \mathrm{PEL}^{\mathrm{B}}$, iesu christi 757677

uobiscum ] cum AMst ${ }^{\text {var }}$ + amen 51 
16:24 Present: 5154586164757677788889 AMst PEL

caritas mea cum omnibus uobis in

in christo iesu ] in christu 54

amen ] om. 64777889 AMst

Explicit

Present: $545^{86164757677788889}$

explicit epistula ad corinthios prima ] explicit ad corinthios 54 , om. 58 , explicit prima epistula ad corinthios 61 , explicit ad corinthios prima 64 77 ad corinthios prima explicit $75^{*}$, ad corinthios prima scribens a philippis explicit $75^{c} 76$, explicit ad corinthios prior 78 , incipit ad corinthios prima 88 , explicit epistula ad corinthios prima 89

\pm habet uersus mille $5488^{\mathrm{C}}$ 